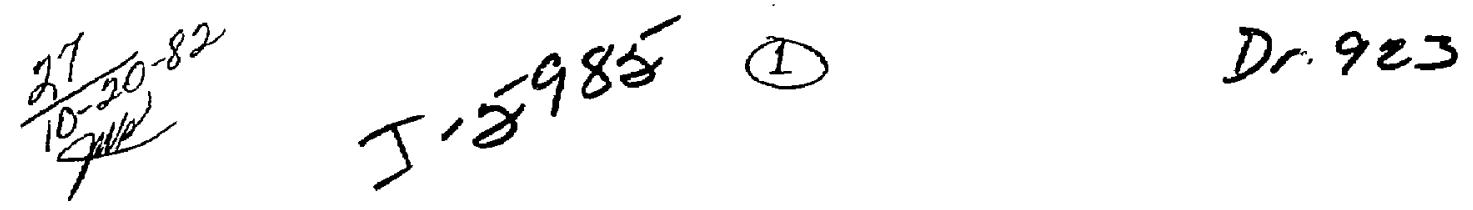

\title{
Mirror Fusion
}

\section{Physics Basis for an Axicell Design for the End Plugs of MFTF-B}

\author{
Editors:
}

D. E. Baldwin

B. G. Logan

April 21, 1982

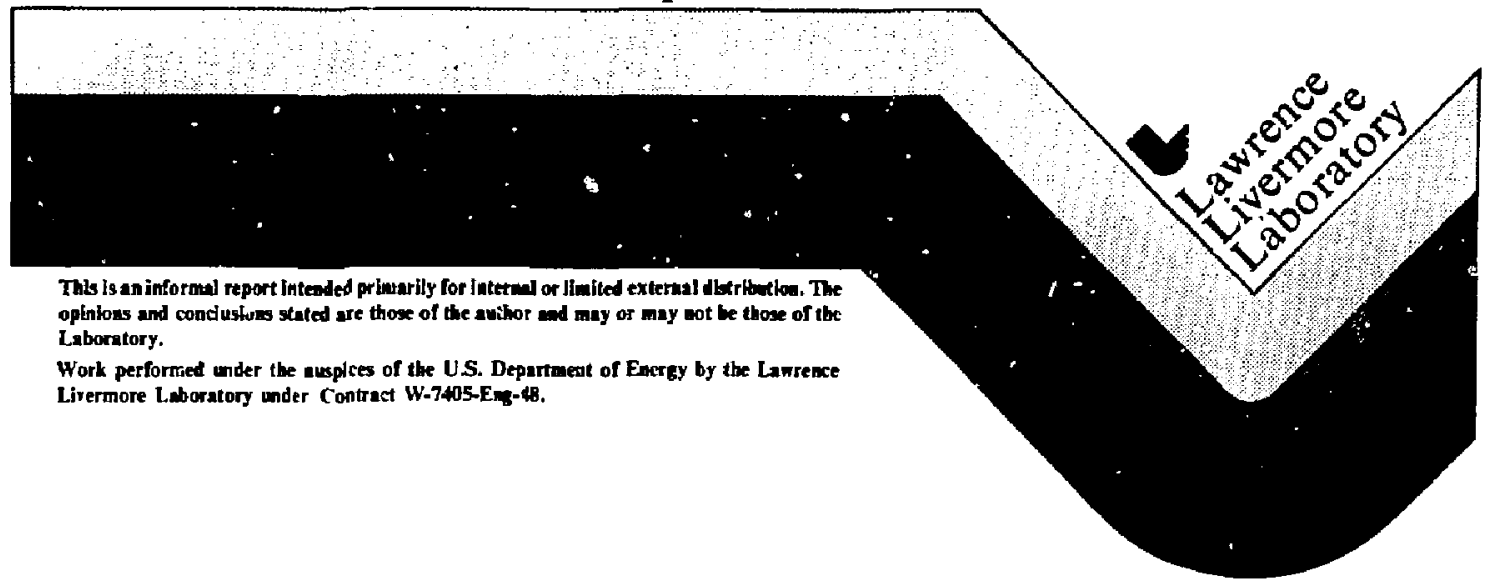

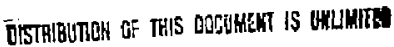




$$
\begin{aligned}
& \text { UCID--19359 } \\
& \text { DE83 } 001161
\end{aligned}
$$$$
\text { UCIO-19359 }
$$

PHYSICS BASIS FOR AN AXICELL DESIGN FOR THE END PLUGS SF MFTF-B

D. E. Baldwin and B. G. Logan

Editors

Apri 1 21, 1982

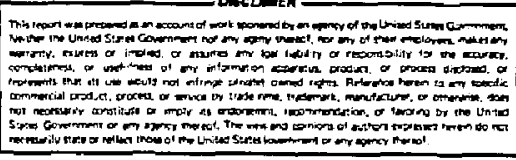




\section{CONTENTS}

List of Figures.......................................... iit

List of Tables................................................ vii

1. Introduction and Physfes Summary ..............................

1.1. Physics Overview .......................................... 1

1.2. Central-Cell Confinement .............................. 5

1.3. Magnetohydrodynamic (MHD) Equilibrium ..................... 5

1.4. Low-Frequency Stab11tty - MHD ......................... 6

1.5. Low-Frequency Stability - Electrostatic .................. g

1.t. Microstability ....................................., 11

References ................................................, 14

2. Scerrarios for MFTF-B Axicel1 Operation........................., 17

2.1. Introduction and Sumary .............................. 17

References ........................................., 23

2.2. Axice11 Reference-Case Operation (MARS Mode) .............. 27

2.2.1. Description of Reference-Case MARS Mode ............, 27

2.2.2. Suitability of Reference Case for

Reactor Operation .............................., 28

2.2.3. Operating Scenario for MARS Mode at Steady State ...., 37

2.2.4. Start-Up Scenario for the MARS Mode .............., $5 j$

References ........................................, 60

2.3. Alternate Operating Modes ............................ 63

2.3.1. Kelley TDF-1ike Mode .......................... 63

2.3.2. TMX-U-1ike Mode ............................ 69

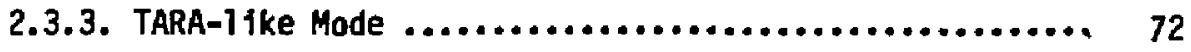

References ........................................ 75

2.4. Physics Models for Calculations of Parameters ............. 79

2.4.1. Central-Ce11 Parameters ........................ 79

2.4.2. Axicel1 Parameters ............................. 79

2.4.3. Barrier Parameters ............................ 82

2.4.4. Warm-Electron and Confining-Potential Parameters ..... 84

2.4.5. Anchor-Ce11 Beam Requirements .................... 88

2.4.6. Microwave Power Requirements ................... 92 
2.4.7. Central-Cell Ion-Particle and Energy Balance ........ 93

2.4.8. Central-Cell Electron-Particle and Energy Balance .... 96

References .................................. 98

Appendices

A. Analytic Equilibria with Quadrupole Symmetry in the

Paraxial Limit ......................................... 103

B. Gyrokinetic Magnetohydrodynamics (MHD) ................. 115

C. Some Effects of Hot-Elertron Stability in

Tandem-Mirror Geometry .............................. 139

D. Electrostatic Ballooning Modes ......................... 147

E. Sloshing lons in the MFTf-B Anchor ................... 163

F. Ion Microstability ............................. 181

G. Electron Microstability ............................... 195

H. Radial Transport ................................... 207

I. Barrier Potential Mode1 ............................... 219 


\section{LIST OF FIGURES}

1-1. Comparison of A-cel1 and axicell designs for MFTF-B. ........ 2

1-2. Field, density, and potential profiles for the MARSmode reference case of the MFTF-B Axicell design. .......... 3

2.1-1. Magnet set for MFTF-B Axice11........................ 18

2.1-2. Thermal-barrier MARS-mode reference case for MFTf-B Axice11. .................................. 20

2.1-3. Improved MARS axice $] l$ reactor. ........................ 21

2.2-1. A-ce)! Tandem-Mirror Reactor (JKR) . ................... 31

2.2-2. Axicell TMR. ...................................... 32

2.2-3. Modified cusp THR. ............................... 33

2.2-4. Typical central-cell particle balance for MARS mode of MFTF-B. ................................... 41

2.2-5. Typical central-cell ion-energy balance for MARS mode of MFTF-B. .................................... 42

2.2-6. Typical centrat-cell electron-energy balance for MARS mode of MFTF-B. ................................ 43

2.2.7. Comparison of passing ions and electrons in MFTF-B yin-yang anchor. ................................. 46

2.2-8. Variation of overall $Q$ and of ion fraction passing into the yin-yang anchor as a function of axicelland pump-beam power. .................................. 50

2.2-9. MARS-mode (vacuum) flute interchange. .................. 52

2.2-10. Operating window during start-up of MFTF-B. ............. 54

2.2-11. Start-up of MFTF-B Axicell with thermal barriers. ........... 56

2.2-12. Approximate central-cell power balance used for start-up. .................................... 59

2.3-1. Axial profiles in Kelley mode of MFTF-B Axice11. ........... 54

2.3-2. Central-cell power flow for alternate modes. ................ 68

2.3-3. Axial profiles in TMX-U mode of MFTF-B Axicel1.............. 70

2.3-4. Axial profiles in the TARA-1ike configuration of MFTF-B. ........................................ 73

2.4-1. Loss boundary and distribution-function contours for sloshing-ion injection in yin-yang anchor. 
2.4-2. Potential and density profjles obtained for sloshing-ion injection in yin-yang anchor. .............. 87

2.4-3. Loss boundary and distribution-function contours

for passing and trapped ions in the transition region. ...... 89

FIGURES IN APPENDICES

B-1. Critical value of $B_{c}$ as a function of $\psi . \ldots \ldots \ldots \ldots \ldots \ldots \ldots \ldots \ldots 120$

B-2. Critical value of $B$ ss a function of $F, \ldots \ldots \ldots \ldots \ldots \ldots \ldots \ldots \ldots, 120$

B-3. Phase-space trajectory and invariant toroio for two degrees of freedom. ................................... 124

B-4. Example of an integrable ballooning ray orbit. .............. 126

8-5. Example of a stochastic orbit. ........................ 127

B-6. Line spectrum characteristic of a system with we 11-separated time scales. .............................. 129

B-7. Marginal stability boundary in the $\beta_{c}, \Omega *$ plane. ............ 132

B-8. A plot of $S_{\theta}$ ys $B_{c}$ at marginal stability. ................. 132

C-1. Frequency dependence of right and left side; of the dispersion relation. ................................ 142

D-1. Field, density, and potintial profiles in the MFTF-B Axicell design, showing worst $E B$ balloonirig mode, which occurs when the density in the transition is very low. ...........

$D-2$. Field, density, and potential profiles in the MFTF-B Axicell design, showing the trial function at low plasma density in the transition.

D-3. Field, potential, and density profiles at one end of MFTF-B when operated with A-cel l-type thermal barriers. .........

E-1. Axial magnetic-field profile in anchor. .................... 164

E-2. Potential profile in anchor. ........................... 166

E-3. Loss-cone boundaries in midplane velocity space of anchor. ............................................ 170

E-4. Ion density as a function o: magnetic field for

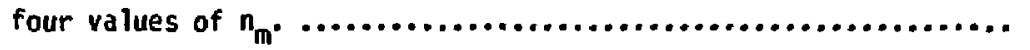


E-5. Contour plots of ion-distribution function, $f_{0}\left(v_{1}, v_{11}\right)$, at anchor midplane for four values of $n_{m} \ldots \ldots \ldots \ldots \ldots \ldots \ldots \ldots . \ldots 174$

E-6. Distribution of perpendicular velocities at anchor midplane for four values of $n_{m}$. ....................... 175

E-7. Distribution of parallel velocities at anchor midplane for four values of $n_{m}$. ............................. 176

F-1. Stability of axicell configuration to 105s-cone modes. ......... 182

F-2. Marginal stability for two worst modes and for configuration with a $20 \%$ broader ECRH profile. ............... 184

$F-3$. Ion-distribution function $f$ as a function of perpendicular veloctty at midplane of anchor at marginal stability. .................................... 186

F-4. Amp litude and phase of the eigenfunction of marginal stability for the $n_{z}=0$ mode and for the $n_{z}=1$ mode. $\ldots \ldots \ldots \ldots$

G-1. Contour plot of the electron distribution found in Fokker-Planck studies of the ECRH plasma in the TMX-U experiment.

G-2. Mode1 electron distribution formed by superposing five separable distributions. 198

G-3. Temporal growth rate of the upper-hybrid loss-cone instability for the electron distribution shown in Fig. 6-2. .... 200

G-4. Growtin rates, maximized over $\underline{k}$, of the whistler and upper-hybrid loss-cone (UHLC) instabilities as a function of the temperature anisotropy.

G-5. Constant-growth-rate curves for the whist ler and UHLC modes.

G-6. Convective-absolute instability boundary for whistier mode.

$\mathrm{H}-1$. Resonant diffusion coefficients, $\mathrm{D}_{0}, \mathrm{D}_{1}, \mathrm{D}_{2}$ and lifetime estimate $\tau_{2}$, for MFTF-B preliminary design, all as a function of electric fieid. 
1-1. Magnetic-field strength and ambipolar potential for a typical thermal-barrier cell in a tandem mirror. .............. 220

1-2. Thermal-electron phase space. ............................ 220

1-3. Thermat-ion phase space. .............................. 222

1-4. Profiles of Mod-B, ambipolar patential, thermal-ion density, and s loshing-ion density for $\alpha=1.5 . \ldots \ldots \ldots \ldots \ldots \ldots . \ldots 228$

I-5. The same profiles as in Fig. I-4 out with $\alpha=3 . \ldots \ldots \ldots \ldots \ldots \ldots .228$

1-6. Various equiliorfum quantities as a function of $g\left(R_{H}\right) \ldots \ldots \ldots . \ldots 230$ 


\section{LIST OF TABLES}

2.1-1. Key parameters for operating modes of MFTF-B with therma 1 barriers. ..................................... 22

2.2-1. Plasma parameters for reactor types. ................... 29

2.2-2. Reactor power balance (a11 powers in megawatts). ........... 34

2.2-3. Reactor costs in millions of dollars (SM). .............. 35

2.2-4. Reactor costs in dollars per kilowatt-e lectric $(\$ / k W e) . \ldots \ldots . . .35$

2.2-5. Key parameters of axicell tandem reactors. ................. 36

2.2-6. Plasma parameters for the MARS mode of the MFTF-B Axicel1. .... 38

2,2-7. Trapped-particle stability parameters for MFTF-B and for axicelt tandem-mírror reactors. .................... 49

2.3-1. Central -cell plasma parameters. ........................ 65

2.3-2. Parameters for MFTF-B Axicel1 in the TARA-like mode (for an earlier design), $Q=0.37, Q_{C}=1$,

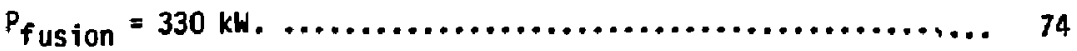

TABLES IN APPENDICES

Table B-1. Critical beta values for the central cel1. .............. 116

Table E-1. Input parameters for sloshing-ion anchor. ............. 171 Table E-2. Effect of passing-ion density on anchor parameters. ....... 172 


\section{PHYSICS BASIS FOR AN AXICELL DESIGN FOR THE END PLUGS OF MFTF-B}

\section{INTRODUCTION AND PHYSICS SLMHARY}

The primary motivation for conversion of MFTF-B to an axicell configuration lies in its engireering promise as a reactor geometry based on circular high-magnetic-field colls (see Ref. 1.1). In comparing this configuration to the previous A-cell yecinetry, we find a number of differences that might significantly affect the physics performance. The purpose of the present document is to examine those features and to assess their impact on the performance of the axice11, as compared to the A-cell configuration, for MFTF-B. In so doing, we address only those issues thought to be affected by the change in geometry and refer to the original report 1.2 mphysics Basis for MFTF-B," for discussion of those issues thought not be affected.

In Sec. ?, we summarize these physics issues. In Sec. 2, we describe operating scenarios in the new configuration. In the Appendices, we discuss those physics issues that require more detailed treatment.

\subsection{PHYSICS OVERYIEW}

The magnet set for the axicell version of MFTF-B is shown in Fig. 1-1. The central cell is terminated at either end by an axisymetric mirror cell called the axice11. This configuration was inspired by the geometry of TARA, which is under construction at MIT. ${ }^{1.3}$ However, to accomodate the MFTF-B reference mode of operation, the axicell in MFTF-B has a lower mirror ratio and a different $B$ profile from TARA.

In the plasma configuration that forms the reference mode (MaRS mode) for MFTF- $B$, the axicell throttles the flow of ions to the end cell. This is done by a combination of magnetic constriction and reflection from a potential peak formed by ions mirror-trapped in the axicell. The final and higher plugging potential peak and the associated thermal barrier are found in the minimum-B anchor, as shown in Fig. 1-2. Those ions confined to the central cell by the axicell see only axisyminetric magnetic and electrostatic fields.

Situated outside of the axicells and separated by transition sections, the MFTF ying-yang magnets act as magnetchydrodynamic (MHD) anchors to the whole system. Electrical contact between the axicell/central-cell combination 


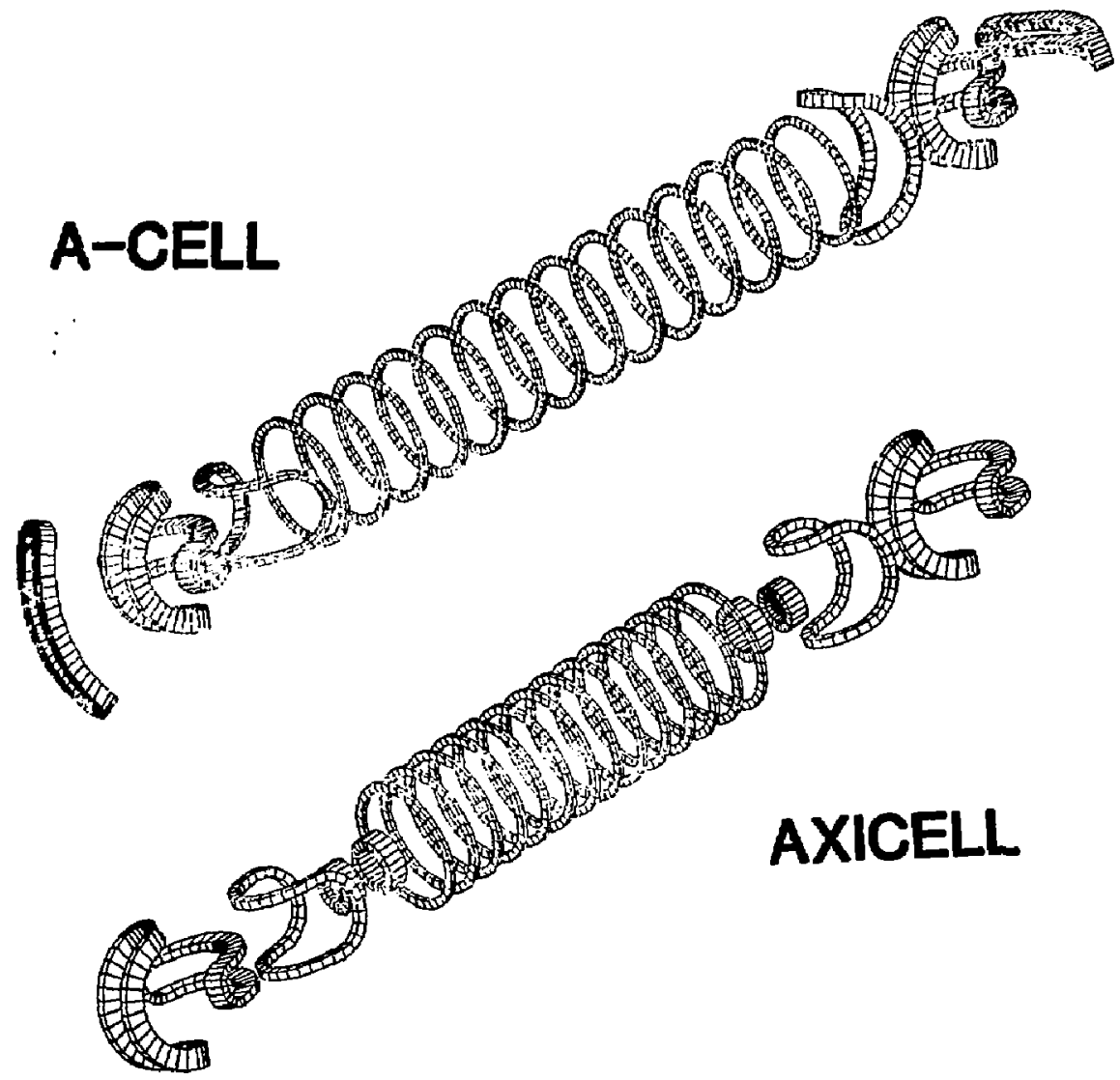

Fig. 1-1. Comparison of A-cell and axicell designs for MFTF-B. 

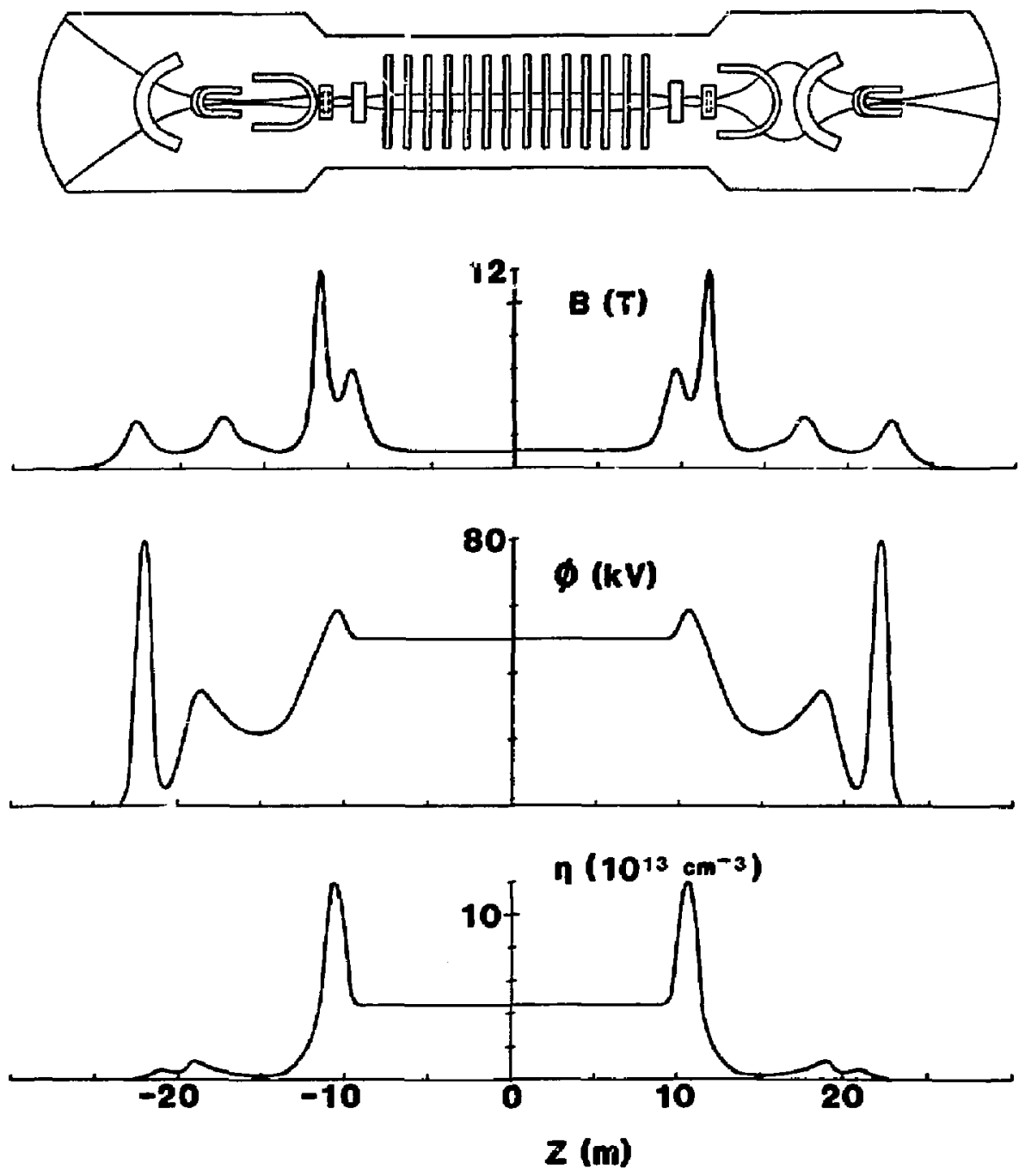

Fig. 1-2. Field, density, and potential profiles for the MARS-mode reference case of the MFTF-B Axicell design. 
and the minimum-B anchor cell is maintained througb the transition region by a plasma of relatively low density and pressure. Model axial profiles for the density and potential are also shown in Fig. 1-2.

In assessing the physics performance of this configuration, we have identified three critical parameters. Their significance is discussed in the following sections, and more detail is given in the Appendices.

One parameter is set by the degree of coupling of the curvature-driven trapped-particle mode in the central cell to a drift wave. A minimum coupling is required for stability. At high azimuthal-mode numbers this comes about by finfte ion Larmor radius effects. For low-mode numbers, there is a coupling induced by details of the charge-particle confinement by the end cells. This sets a minimum for the fraction of central-cell ions that are confined by tine end ceil. This constraint is met in the HFTF-B design and is less restrictive in reactor extrapolations. For MFTF-B, about $2 \%$ of the central-cell ions are confined by the outer potential peat: in the reference design. With this fraction, MFTF-B is calculated to be marginally stable to the trapped-particle mode.

Another critical parameter is the achievable $B$-value in the central cell as determined by both equilibriun and stability considerations. An important new element in determining the central-cell $\beta$ 1 imit is the density (and therefore $B$ ) in the transition between the yin yang and exicell regions that is required to stabijize the trapped-particle mode. The final magnetic-design is not yet complete, particularly regarding the minimization of the parallel currents in the equilibrium. Even so, with an intermediate design we find that centra!-cell b-values ranging from $36 \%$ in the equilibrium field to 533 in the vacuun field can be sustained and are consistent with an anchor $B$ of $55 \%$ and axicell $B$ of $20 \%$.

The third critical parameter is the ratio of the censity of warm ions that penetrite into the midplane of the yin-yang anchor to the density of energetic sloshing ions that possess a loss cone. Such a caexistence of warm and hot ions is necessary for stability of the latter to loss-cone modes. We find that a warm-ion midplane-density fraction $\geq 0.6$ at a $T_{\text {warm }} / T_{\text {hot }}$ ratio $\geq 0.3$ should be nominally stable, as compared to the reference-case design parameters where $n_{\text {warm }} / n_{\text {hot }}=0.8$ and $T_{\text {warifi }} / T_{\text {hot }}=0.32$. 


\subsection{CENTRAL-CELL. CONFIMEMENT}

From the point of view of confinement physics, a primary advantage of the axicell configuration is the reduction of radial transport in the central cel1. The dominant radial-loss mechanism in the original MFTF-B central cell was a resonant transport of ions having half-integer-related axial bounce and azimuthal-drift frequencies and suffering radial displacements because of the nonsymmetric field components. In the axicell geometry, only the passing particles experience nonsymmetric fields. Most of the central-cell ions see symmetric fields so that their transport is classical in the absence of symmetry-breaking field errors or fluctuations. Ions entering the end regions suffer radial steps because of the same geodesic curvature that drives parallel currents. Once the parallel current is largely eliminated (a design constraint), the overall transport will be reduced compared to the A-cell. Detaiis of this improvement are given in Appendix $H$.

\subsection{MAGNETOHYDRODYNANIC (MHD) EQUILIBRIUM}

Finite- $\beta$ equjlibria in nonaxisymetric mirror qeometries are dominated by the currents parallel to $B$, which are generated by the nonvanishing divergence of the dianagnetic current. These currents are analogous to the Pfirsch-Schluter currents in tokamaks and produce closed plasma-curren: 10ops that can roughly image the external yin-yang or baseball magnet currents. In a multicell system such as a tandem geometry, these loops can internally close over either the full axial extent of the system as a whole or separatily in each cell.

A tandem-mirror system with a long central cell must have small quadrupole axial current in the central cell. Otherwise it would generate ${ }_{B}, B_{r}$, and large flux-tube distortions proportional to the central-cell length. An important constraint on a magnet-set design is that all current loops must at least close in each end region. Our reference design satisfies this condition. A much preferred solution has current loops closing separately in each mirror cell of an end region, which avoiss a required balancing of adjacent cell properties, particularly pressures, and permits a wider parameter space of central-cell axial-current-free operation. We also have an 
in-principle solution that meets the latter more stringent condition but do not as yet have a satisfactory design from the engineering point of view.

Our design code for this problem is TEBASCO, which solves for plasma equilibrium to linear order in $B$, using magnet fields generated by our engineering magnet design code EFFI. TEBASCO is routinely used by the magnet design team to iterate magnet designs to adjust the central-cell axial current to zero while satisfying acceptable flute-stability criteria

$[B$ (central-cell) $>B(p] u g)]$, Detalls of the theory and examples of the code results are given in Appendix $A$.

\subsection{LOW-FREQUENCY STABILITY - MHD}

Hodes in the drift-frequency range are conveniently distinguished by (1) whether the parallel electric field $\tilde{E}_{\|}$vanishes by virture of an inductive vector potential $\tilde{A}_{n}$, cancelling the longitudinal field $a \tilde{\phi} / \partial s_{\text {, }}$ or (2) whether $\widetilde{E}_{1 !}=-\partial \tilde{\phi} / \partial s$ can exist in spite of the pressure plasma. The first is characteristic of MHD behavior, with or without finite Larmor ratius (FLR) corrections. The second is characteristic of electrostatic ballooning behavior, which is very similar to that of trapped-particle modes in tokamaks.

When calculated by pure MHD theory, the axicell configuration has a central-cell B limit even below that of the A-cell configuration. The worst modes in this description are highly localized, bending the thin dimensions of the fan-shaped region between the plug and anchor with little expenditure of bending energy. In the long solenoid, FLR has a strong stabilizing effect on such highly localized modes. When the worst modes have large enough azimuthal variation to justify an eikonal approximation, the full 3-D FLR stability problems can be analyzed using the procedures described in Appendix B. However, these calculations show such a strong FLR stabilization that, for most parameters of interest, only fery low azimuthal-mode numbers could be unstable; these, of course, lie outside the validity of the theory.

A proper treatment of this problem requires a full 3-0 stability analysis, including the nonsymmetric anchors and rotation in the central cell. This theoretical capability is currently under development at LLNL. Lacking this tool to date, we neglected the high-m modes and used the flute-interchange stability condition as a figure of merit in magnetic-field design. 
Recent PHAEDRUS results support our neglect of high-m modes ${ }^{1.4}$. In that experiment, only $m=1$ was observed, and the stability limits agreed with those of the flute coridition. Beta values were too low to expect ballooning in PHAEORUS, but finite $B$ experiments in the Wisconsin octupole $e^{1.5}$

demonstrated increased ballooning $\beta$ limits consistent with FLR theory.

The low-m stability rroblem is complicated by several features, in addition to the 3-D character of the equilibrium, namely:

- A powerful energy principle, as in MHO, is no longer available so that eigenmoces and frequencies must be calculated.

- An eikonal treatment is no longer valid.

- The rotation drive in the central cell occurs in the same order as FLR.

- The mode structure is richer, particularly in the presence of hot electrons as described in the following.

As just discussed, our analysis so far has been eikonal, and the curvature-driven response has been treated in the high-frequency limit, as in MHD. In a number of important circunstances, this analys is remains valid in the presence of fast drifting hot electrons if the curvature-driven response is properly modified. This procedure is outlined in the following and detailed in Appendix $C$.

Following the idea developed for the TARA tandem mirror at MIT $T^{i .3}$, the anchor cells in MFTF-B will derive most of their pressure from an anisotropic population of hot electrons. Ample experience in the Elmo Bumpy Torus (EBT), ELMO, and INTEREM experiments demonstrates that local B-values of several tens of percent can be generated with relatively low-power electron-cyclotronresonance heating (ECRH) and a considerable parameter space of (electron) microstable operation.

Because of their fast precessional drift frequencies, these electrons do not behave as an MHD fluid and, therefore, would not by themselves contribute a positive pressure weighting to, for example, a flute-stability integral.

These electrons also should be "rigid" in a minimum-B anchor and not contribute to stability under conditions similar to those where electron rings in EBT are "rigid" (i.e., do not have an unstable HHD response in a simple mirror). However, consistent with EBT results, the warm (or slow-drifting) plasma coexisting with the hot electrons has an MHD-like response in the well that is dug (or enhanced) by the hot electrons. The anchor $B$ value is divided 
between a hot component $\beta_{h}$ and a lower pressure warm component $\beta_{W}$ ("hot" and "warm" being defined by frequencies of species' drift relative to the central-ceIl MHD frequencies). The contribution to flute stability is given by

$$
\int \frac{d \ell}{r} k\left(\frac{\beta_{w}\left(\beta_{h}+\beta_{w}\right)}{\beta_{w}+2 r_{n} k}\right),
$$

where $r_{n}>0$ is the density scale length, and $k$ is the component of curvature normal to the pressure-level surfaces. For $\beta_{W} \gg 2 r_{n} k$, this expression becomes

$$
\int \frac{d \ell}{r} k\left(B_{h}+B_{w}\right) \text {. }
$$

which is the appropriate weighting when all species are treated as having an MHD response. For finite $B_{w} / 2 r_{n} k$, the region containing hot electrons can still be treated as if it had an MHD response, provided the local $\beta$-value is degraded by a factor $\beta_{w}\left(\beta_{w}+2 r_{n} k\right)^{-1}$. (In this example flute perturbation is assumed, but a similar result would hoid for a ballooning perturbation.) Thus, by this replacement, eikonal balloon-code results, including FLR as the only kinetic effect, can describe tandem systems that have anchors containing fast-drifting electrons.

This change in behavior with $\beta_{w_{6}}$ compared to $2 r_{n} k$ is directly analogous to the Van Dam-Lee-calculated 1 imit ${ }^{1.6}$ on the core $\beta$ in EBT. There $k<0, s$, the denominator and, therefore, the sign of the response changes as $\beta_{k}$ exceeds $12 r_{n} \mathrm{kl}$. The emergence of an MHD response for the hot electrons in a negative-curvature well, therefore, implies instability; whereas in a positive curvature well it implies increased anchoring.

Our picture of low-frequency stability can be sumarized as follows. Finite Larmor radius in the central cell stabilizes all but vary low-m modes. A proper three-dimensional theory for these has not yet been developed. In our magnet design, the flute-interchange condition is being used as a figure of merit with experimental support. If appropriate effective $B$ values are used, and even with hot electrons in the anchor, ballooning and flute-avr.rage calculations can be carried out. 


\subsection{LOW-FREQUENCY STABILITY - ELECTROSTATIC}

The electrostatic ballooning modes, first identified by Berk ano

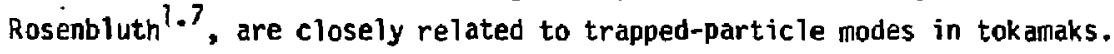
Because of their recent arrival to the tandem-mirror scene, we give more detail to their properties in this overview.

In such an instability, particles trapped in regions of bad curvature can drive an electrostatic perturbation that excludes good-curvature regions. Particles passing between the two regions can Debye-shield the perturbed potential, but this is a fairly weak effect if only a small fraction of particles are passing.

When the electron-bounce frequency throughout the machine length greatly exceeds the wave frequency, the resulting nonvanishing $\widetilde{E}_{11}$ toes not drive a parallel electron current, as it would do in an inf inite medium.

Because all tandem mirrors only have good magnetic curvature in a line-averaged sense, they are predicted to be susceptible to such modes, as are tokamaks. The tandem-miror central cells are separated from the end cells by large mirror ratios--10 or more for experiments and about 5 for a reactor--and the passing-particle fraction is small by at least the inverse of these airror ratios. For such passing fractions, the calculated electrostatic-mode growth rates are relatively small, the azimuthal m-values relatively large, and the modes are perhaps benign. Hoxiever, thermal-barrier efficiency can be increased by further decreasing the passing-particle fraction. Berk and Rosenbluth ${ }^{1.7}$ showed that, in the exireme of such cases, the electrostatic mode growth rate can rise to that of the MHD value of the central cell alone, and the m-value can drop to unity = In such a circumstance, tine central cell would be expected to tear loose from the anchors until limited by a noninear process. The severity of this worst-case scenerio for electrostatic modes warrants paying close attention to their properties to find means of stabilization.

The stability of the electrostatic ballooning mode can be modelied by the quadratic equation, as shown in Appendix $0,1.8,1.9$

$$
\left(1+\frac{A_{1}}{m^{2}}\right) \omega^{2}-\omega m \omega_{* i}\left(\frac{m-1}{m}+\frac{A_{2}}{m^{2}}\right)+\gamma_{c}^{2}=0,
$$


where $m$ is an azimuthal mode number, $w_{k_{j}}$ is the ion-diamagnetic-drift frequency, and $\gamma_{c}^{2}$ is the MHD flute-averaged pressure-weighted growth rate including all regions except the minimum- $B$ anchors. The (m-1) term is the familiar FLR-stabilizing effect. The coefficients $A_{i, 2}$ are enfigurationand geometry-dependent: $A_{1}$ measures the Debye-shielding because of particles passing between regions of vanishing and nonvanishing perturbed potential; $A_{2}$ measures the difference between the bounce-averaged, perturbed $E \times B$ drift of ions and electrons and takes the sign of the charge having the lowest such drift speed. This difference does not occur in tokamaks but does in tandem mirrors where there is an important electrostatic aspect to parallel confinement separating the turning points of positive and negative charges. The coefficient $A_{2}$ can support or cancel the FLR term, depending on its sign. Cancellation when $A_{2}<0$ leads to electrostatic instability at sonie value or range of $m$. Preferred designs use $A_{2}>0$. When this is the case, the worst mode is usually $m=1$, and stability then requires

$$
\frac{w_{*}^{2}}{r_{c}^{2}}>\frac{4\left(1+A_{1}\right)}{A_{2}^{2}}
$$

In the axicel1 design,

$$
A_{1}=A_{2}\left[\frac{r_{c}^{2}}{\rho_{j}^{2}} \frac{n_{\text {pass }}}{n_{c}} \frac{2}{L_{c}} \frac{B_{c}}{B_{a}}\left(L_{a}+L_{t}\right)\right] \text {, }
$$

where $r_{c} / D_{j}$ is the number of ion Larmor radii in the central cell; $L_{c}, L_{t}$, and $L_{a}$ are, respectively, the central-cell, transition, and anchor lengths. For $L_{c}=16 \mathrm{~m}, L_{t}=5 \mathrm{~m}, L_{a}=2.5 \mathrm{~m}$ in the MFTF-B axice 11 design, and

$$
A_{1,2}=0.56 \frac{r_{c}^{2}}{p_{j}^{2}} \frac{n_{\text {pass }}}{n_{c}}
$$

Thus, taking values for MARS-mode of MFTF-B described in Sec. $2, r_{c} / \rho_{i} \approx 13$, a $r$.io $n_{\text {pass }} / n_{c}>5 \%$ is stable for $\omega_{*_{i}}^{2} \approx \gamma_{c}^{2}$, whereas $n_{\text {pass }} / n_{c}>1.6 \%$ is stable for $\omega_{* i}^{2} \approx 2 \gamma_{c}^{2}$. The parameter $A_{1,2}$ is the important physics parameter determining the coupling to the anchor. In the MFTF-B Axicell design, this ratio can be varied by about 100 through variation of $r_{c} / \rho_{i}$ and $n_{p a s s} / n_{c}$. 
In the original A-cel1 design, the worst perturbation is one that eliminates only the yin yang. Both ions and electrons have access to the yin yang, although the ions are depressed by a potential $\Delta \varphi_{p}$ resulting from neutral-beam injected ions trapped in the yin yang. The coefficient $A_{1}$ takes the form

$$
A_{T}=\frac{1}{R_{c}}\left(\frac{T_{i c}}{T_{e c}}\right)+e^{-\Delta \varphi_{p} / T_{i c}}\left(\frac{r_{c}^{2}}{\rho_{j}^{2}}\right) \frac{2\left(L_{a}+L_{A}\right)}{L_{c}},
$$

when $R_{c}$ is the central-cell mirror ratio, $T_{i c}$ and $T_{e c}$ the central-cell temperatures, and $L_{a}$ is the effective yin-yang (anchor) length. The coefficient $A_{2}$ is negative because of the free penetration of the central-cell electrons into the anchor,

$$
A_{2}=-\frac{1}{R_{c}}\left(1-e^{-\Delta \Phi_{p} / T_{i c}}\right) \frac{r_{c}^{2}}{\rho_{i}^{2}} \frac{2\left(L_{a}+L_{A}\right)}{L_{c}} .
$$

If we use $L_{c}=32 \mathrm{~m}, R_{c}=4, T_{i c} / T_{e c}=1.7, L_{A}=4 \mathrm{~m}$, and $\Delta \varphi \approx T_{i c}$, and the parameters above, we find $A_{1} \approx 38$ and $A_{2} \approx-12$. Thus, by $E q .1 .2$, we would expect an instability with $m \approx 3$.

In the absence of charge-separation effects, obtained by setting $\omega_{\star_{j}}=0$ in Eq. 1.2, all magnet designs would yieid an unstable mode with growth rates differing only by $A_{1}^{-1 / 2}$ for fixed $m$. This is analogous to the situation in tokamaks, except $A_{1}$ is usually much larger in tokamaks if onjy because of the increased number of Larmor radii in the cross section.

In sumary, as in other average-minimum-B-stabilized systems, localized electrostatic ballooning, resulting from particles trapped in regions of unfavorable curvature, appears to be a distiuct possibility. In a tanden mirror, there is a charge-separation mechinism that couples the basic aode to a drift wave. By this means, the mode can be stabilized with parameter constraints compatible with good confinement.

\subsection{MICROSTABILITY}

Instabilities near the icn-cyclotron frequency or its multiples have been a predominant feature of all neutral-beam-heated mirror machines. Control of these instabilities in the plugs and anchors must be considered the 
primary physics challenge in the development of a tandem-mirror fusion reactor. As the tandem-mirror concept has come to employ a magneticaily trapped subpopulation of energetic electrons, the microstability of this group becomes equally important. However, the relatively increased stability of electrons (as opposed to ions) observed in the EBT experinent lends hope that this latter protilem may be easier to solve.

We identify two possible drives for instability: first, the "loss-cone" drive due to an inverted perpendicular energy $\left(E_{\perp}\right)$ population; and second, anisotropy, the excess of perpendicular energy over parallel energy $\left(E_{\| \prime}\right)$ (directions are measured relative to $\underset{2}{\text { ) }}$. Although there are similarities of treatment, it is convenient to discuss ion and electron modes separately.

As regards the ion microstability issue in MFTF-B, we find little difference between the original A-cell design and the axicell design if the yin yang is stretched to produce a vacuum mirror ratio of three. In both cases, the barrier and plugging potentials exist in the same mirror cell. The outer lobe of the sloshing-ion distribution has hotter electrons and consequently a higher potential peak. Ions from the central cell that are confined by this outer peak will supply more than enough warn ions to stabilize the sloshing hot ions at the cell midplane to loss-cone modes. Only near the potential peak does an energy inversion appear, and any modes that might appear are resonant there. A description of Fokker-Planck studies of sloshing-ion distribution is given in Appendix $E$.

The most sensitive region for ion modes is the yin-yang anchor. As discussed in Appendix $F$, we find that stability to loss-cone modes can be achieved within the range of operating parameters. However, an extreme sensitivity of calculated stability boundaries to axial equilibrium profiles is indicative of the theoretical uncertainty. The axicell also contains hot jons, but the distribution is always made monotonic in perpendicular energy because of the passing ions confined by the outer potential peak.

The principal anisotropy-driven mode, the Alfvën ion-cyclotron mode (AIC), has been fairly firmly identified in TMX. Its presence there is well explained theoretically by the extreme anisotropy induced by the norma] injection. As described in Appendix $F$, we find the low $B$ value and sloshing nature of the ions in the anchor to be very stabilizing. The most susceptible regions for this mode are the axicell and the central cell under intense neutral-beam heating. Maintenance of Jow $\beta$ in the axicell and the very large 
mirror ratio of the central cell are both stabilizing, but detailed stability analyses of Fokker-Planck solutions remain to be done. This problem is virtually unchanged by the magnet-design change.

We identify two modes of concern in the electron-cyclotron-frequency

range: first, the upper hybrid mode of the bulk electrons, driven by a nonmonotonic hot-electron population (essentialiy a loss-cone mode); and, second, the whist ler mode, driven by the anisotropy. Our analys is (described in Appendix G) has to date been nonrelativistic, care being taken in matching Fokker-Planck solutions by a series of analytic functions. We find a rather small parameter span for stability, and we calculate fairly short convective-growth lengths in typical Fokker-Planck profiles. At issue is the narrowness of the hot-electron distribution, relative to that of the sloshing ions, that is required to assure good barrier formation. This problem hoids for both axicell and A-cell configurations. Relativistic effects are known to be stabilizing and need to be included in the description. 
1.1. K.I. Thomassen and Y.N. Karpenko, An Axjce 31 Design for the End-Plugs of MFTF-B, LLNL Report, UCIO-19318 (1982).

1.2. D.E. Baldwin, B.G. Logan, and T.C. Simonen, eds., Physics Bas's for MFTF-B, LLNL Report, UCID-18496 (1980).

1.3. J. Kesner, R.S. Post, B.D. McVey, and D.K. Smith, A Tandem Mirror with Axisymetric Central Cell Ion Confinement, MIT Report, PFC/JA81-11 (1981); to be pubtished in Nuc1. Fusion.

1.4. A.H. Molv1k, et a1., Phys. Rev. Lett. 48, 742 (1982).

1.5. S.C. Prager, Bul1. An. Phys. Soc. 26(7), 951 (1981).

1.6. J.W. Van Dam and Y.C. Lee, Proceedings of the Workshop on EBT Ring Physics, Oak Ridge, TH, 471 (1979).

1.7. H.L. Berk and M.H. Rosenbluth, private communication.

1.8. M.N. Rosenbltith, H.L. Berk, H.V. Wong, D.E. Baldwin, and T. Antonsen, "Fast-Growing Trapped Particle Modes in Tandem ivirrors", presented at 1982 Sherwood Meeting, Annual Controlled Fusion Theory Conference, Santa Fe, NM, Apri1 25-28, 1982 .

1.9. L.O. Pearlstein, D.E. Baldwin, R.H. Cohen, T.K. Fowler, and B.G. Logan, "Stabilization of Tandem Mirror Trapped Particle Modes by Incomplete Cancellation of Trapped Particle Drifts", presented at 1982 Sherwood Meeting, Annual Controlled Fusion Theory Conference, Santa Fe, MM, April $2 \overline{5}-28,1982$. 



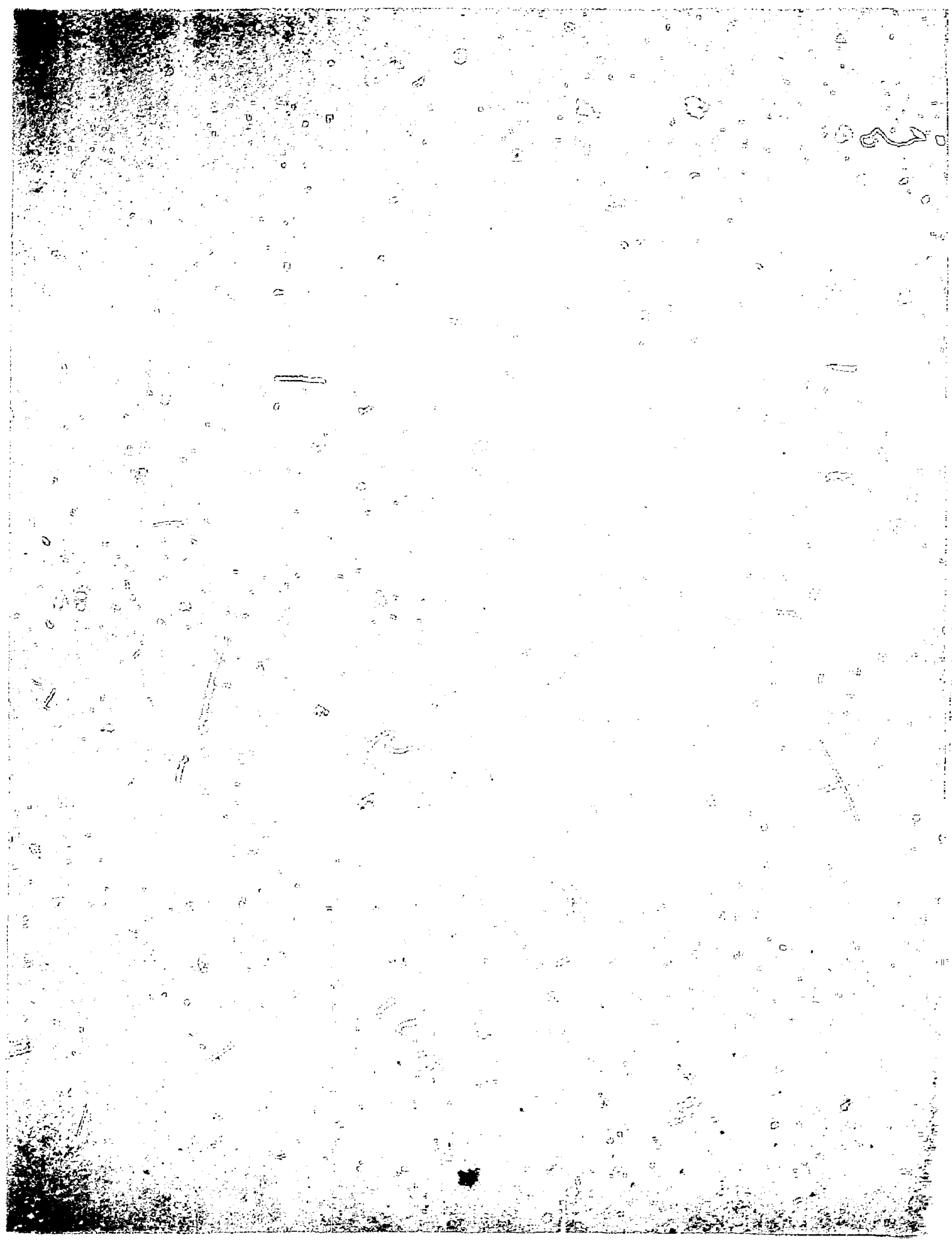


B. G. Logan, R. A. Jong, D. L. Correll, and A. W. Molvik

\subsection{INTRODUCTION AND SUMHARY}

The primary motivation for the proposed modification of MFTF-B to the axice11-plug-magnet configuration ${ }^{2.1}$ (shown in Fig. 2.1-1) is that, compared to the A-cell, this configuration when scaled to a reactor 2.2 is expected to improve performance in terms of higher $Q$, lower plug-magnet capital cost, and reduced radial transport. These reactor improvements are reviewed briefly in Sec. 2.2.2. Using essentially the same plasma models described in the original MFTF-B propn- $?^{2} .3$ but updated where necded (Sec. $i$ ), we estimate that operation with thermal barriers (Sec. 2.2 following) in the modified axicell magnet set would achieve the same physics objectives and equivalent performance as the original A-cell design. Specificaily, we can do the following:

(1) Generate maximum center-cel1 ion-confining potentials ( $\phi_{C} \sim 30 \mathrm{kV}$ ) sufficient for confinement, $(n \tau)_{c} \imath 5 \times 10^{13} \mathrm{~cm}^{-3} \cdot \mathrm{s}$, equivalent to achieving $Q \sim 0.5$ with $D-T$.

(2) Achieve sufficiently high plasma temperatures $\left(T_{i c} \approx 15 \mathrm{keV}\right.$, $\left.T_{e c} \approx 9 \mathrm{keV}\right)$ to test physics of thermal barriers and radial transport in appropriate collision less reactor regimes.

(3) Demonstrate high beta MHD equilibrium and stability $\left(\beta_{c} \sim 0.5\right)$.

(4) Design for microstable sloshing ions and thermal barriers in the MFTF-B yin yangs, using theoretical mujels developed for the A-cel1 design and $T M X-U$.

In addition to the abova physics-design criteria, we also require the operating scenarios for the MFTF-B Axicell to satisfy presentiy understood theoretical criteria for trapped-particle stability 2.4 and to insure $A H D$ response of hot electrons (Lee-Van Dam constraints) ${ }^{2.5}$, even though these theoretical criteria are still evolving and, therefore, are not yet quantitative. Indeed, consideration of trapped-particie stability led to designing the thermal barrier in the yin yang rather than in the axice11, so that more central-cell passing ions can reach the stabilizing yin-yang region. He balieve this is the best mode of operating the MFTF-B Axicell in 


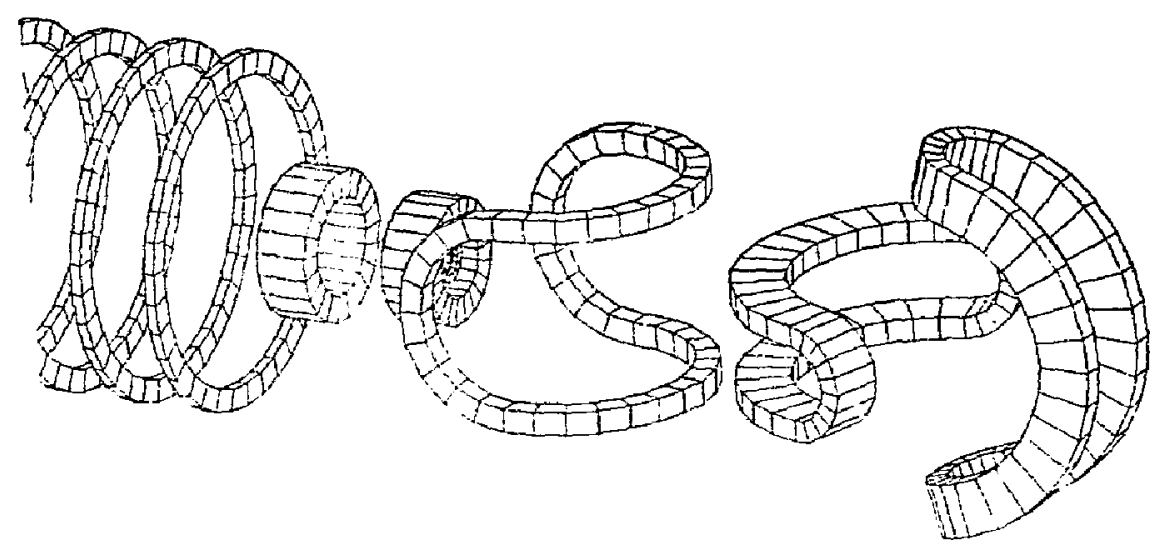

Fig. 2,1-1. Magnet set for MFTF-B Axicel1. 
the on-going Mirror Adyanced Reactor Study (MARS), so we can reter to it simply as the thermal-barrier MAK-mode of MFTF-B. This reference-case operating mode is discussed in detail in Sec. 2.2. Description of the plasma model used for this mode is detailed in Sec. 2.4. In Figs, 2.1-2 and 2.1-3 we compare axial profiles of fie]d, density, and potential for the MARS-mode of MFTF-B and for the MARS reactor, respectively.

In a two-component mode of operation, one can achieve an equivalent $Q>0.5$ with lower confining potentials and, therefore, with relaxen beam-species-mix requirements. This alternative-operation mode uses the same magnet coils with 0.5 -s beams, mostly injected into the central cell. The thermal-barrier potentials are one-half of those in the reference-case MaRS mode (Sec. 2.3.1). Figure 2.3-1 of that section shows the axial prafiles of fieid, potential, and density for this case, which are very similar to those used in the present design of the Technology Demonstration Facility (TDF) engineering-test reactor. The plugs only augment the basic mirror confinement in the central cell, as originally suggested by kelley. 2.6 Thus, we refer to tinis case as the Kelley-TOF-like mode.

To allow for uncertainties in low-frequency stability (particularly the trapped-particle stability), we have also considered (in Sec. 2.3.2) a backup mode of running the axicell MFTF-B magnet set without injecting the axicell or pumping the transition region, so that a plasma configuration very similar to the TMX-U can be obtained (see Fig. 2.3-3). With respect to ballooning MHD and trapped-particle stability, this mode of operation (naturaliy called TMX-U-7ike mode) is probably at least as stable as the original A-cell design of MFTF-B. In fact, one can operate MFTF- $B$ almost continuously between the TMX- $U$ and the MARS modes and obtain increasing central-cell density, increasing Q, and decreasing margin of MHD and trapped-particle stability when going toward the MARS-mode. This continuum of operating points is discussed further in Sec. 2.2. A summary of key parameters comparing these various operacion modes of MFTF-B is given in Table 2.1-1.

Finally, in Sec. 2.3.3, we discuss the option of operating MFTF-B with thermal barriers in the axicell if the axicell coils are spread apart to abtain a larger mirror ratio. This would generate a plasma configuration (see Fig. 2.3-4) similar to the original proposed TARA experiment. 2.1 Operation in this mode is possible only if trapped-particle stability is much better 


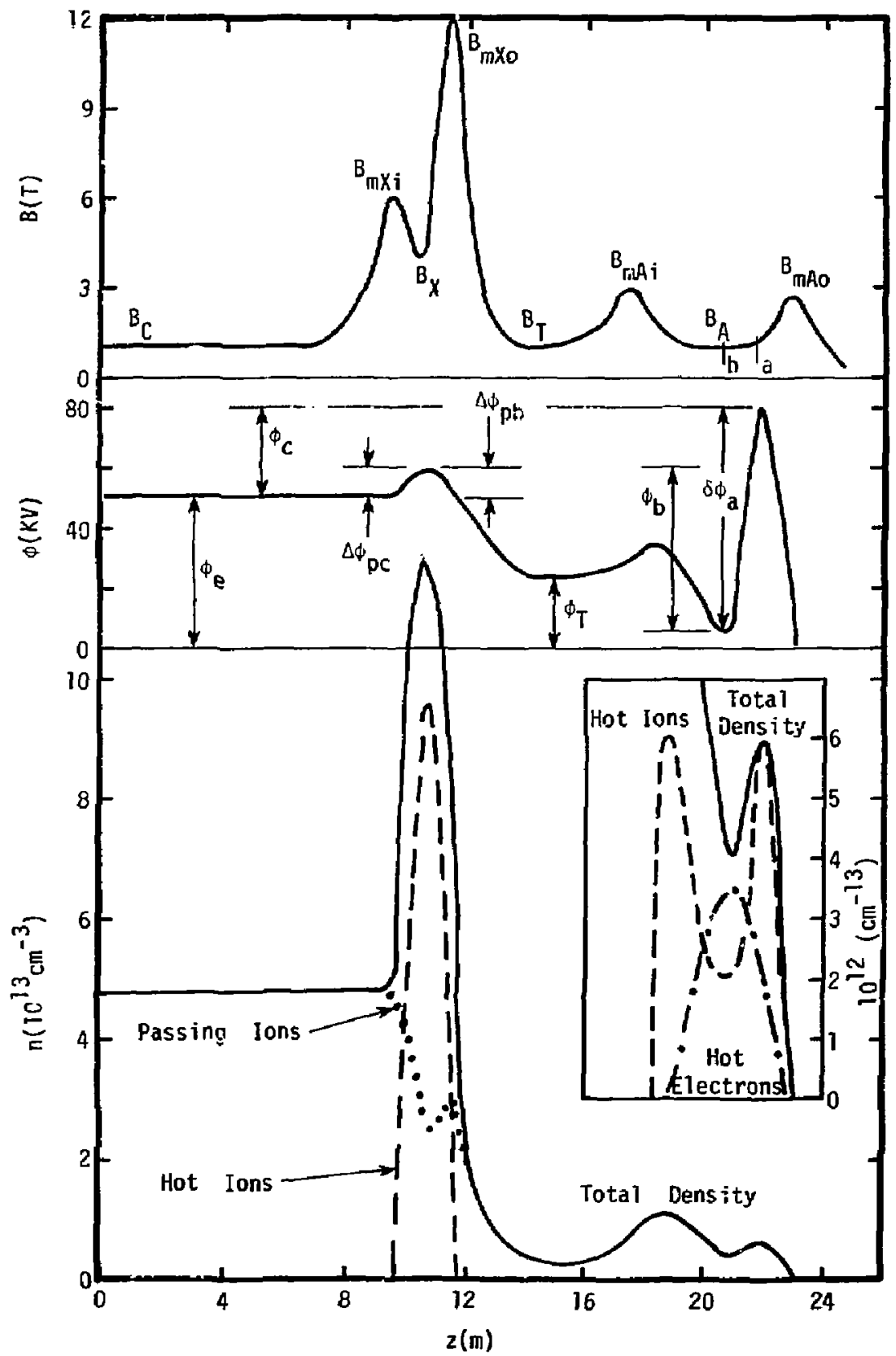

Fig. 2.1-2. Thermal-barrier MARS-mode reference case for MFTF-B Axicell. 


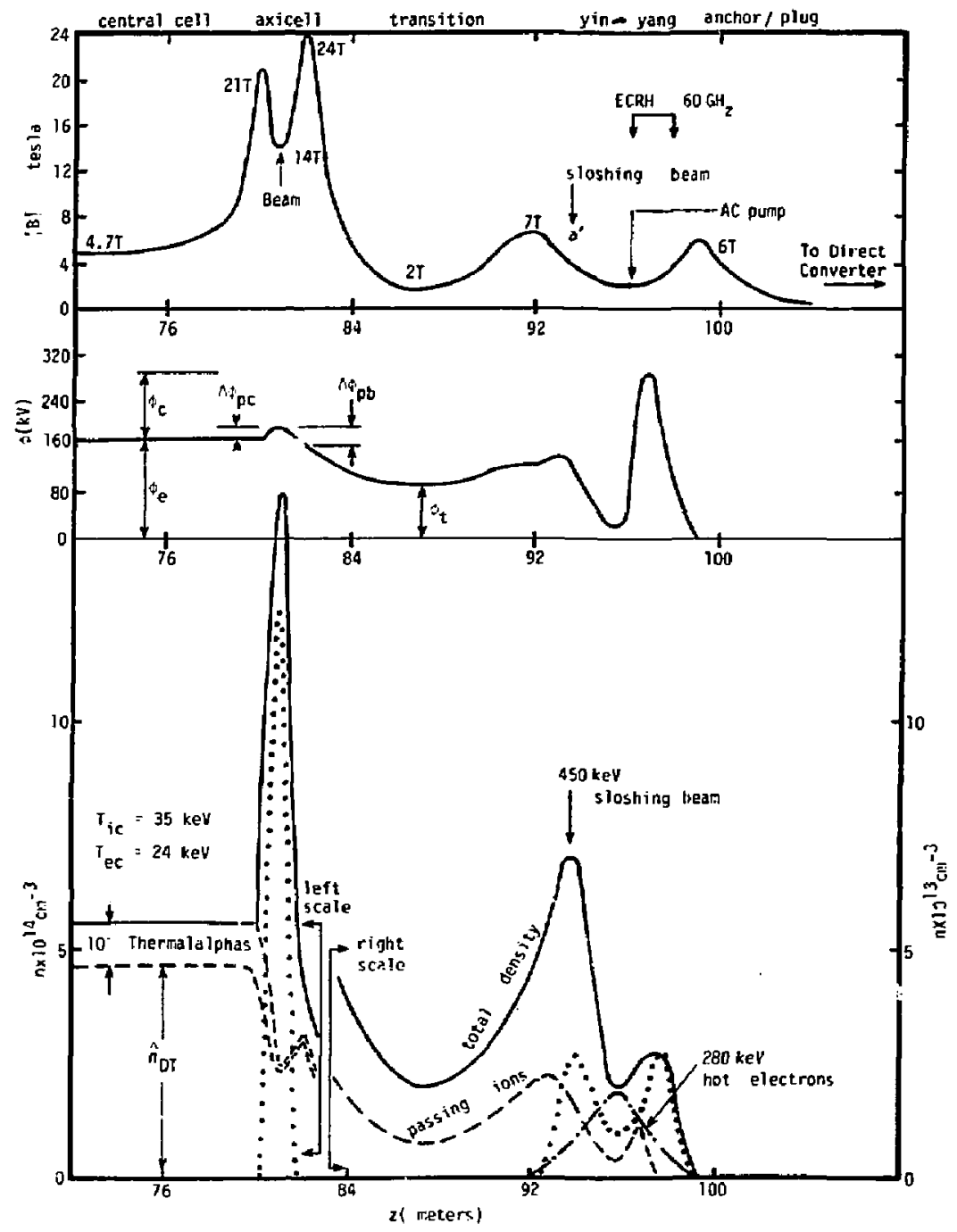

Fig. 2.1-3. Improved MARS axicell reactor. 
TABLE 2.1-1. Key parameters for operating modes of MFTF-B with thermal barriers.

\begin{tabular}{|c|c|c|c|}
\hline \multirow[b]{2}{*}{ Parameter } & \multicolumn{3}{|c|}{ Mode } \\
\hline & MARS.like & TOF-like & THX-U like \\
\hline$Q\left(\right.$ overall) ${ }^{a}$ & 0.40 & 0.6 & 0.06 \\
\hline$T_{\text {ihc }}(\mathrm{keV})$ & - & 35 & - \\
\hline$T_{\text {iwc }}(\mathrm{keV})$ & 15 & 10 & 15 \\
\hline$T_{e c}(k e V)$ & 9 & 6 & 9 \\
\hline${ }^{B} \mathrm{C}(T)$ & 1.0 & 1.6 & 1.0 \\
\hline${ }^{B} \mathrm{C}$ & 0.5 & 0.54 & 0.12 \\
\hline${ }^{n_{C}}\left(\mathrm{~cm}^{-3}\right)$ & $4.8 \times 10^{13}$ & $9.1 \times 10^{13}$ & $1.2 \times 10^{13}$ \\
\hline$(n \tau) C^{\left(c m^{-3} s\right)}$ & $5 \times 10^{13}$ & $1.4 \times 10^{13}$ & $5 \times 10^{13}$ \\
\hline$P_{\text {fus }}(k W)$ & 830 & 4200 & 80 \\
\hline
\end{tabular}

aquivalent for $\mathrm{D}-\mathrm{T}$. 
than we now est imate. The magnet set for use with therma 1 barriers in the yin yang (MARS mode) is designed so that the MFTF-B can be operated in a TARA mode with only a 3-month turnaround (during which the axicell coils are spread and beams and ECRH rearranged).

\section{REFERENCES}

2.1. J. Kesner, Nuclear Fusion 20, 557 (1980). See also J. Kesner, R. S. Post, D. D. McVey, and D. K. Smith, "A Tandem Mirror with Axisymetric Central Cell Confinement," to be published in Nuclear Fusion; also see MIT Rept. PFC/JA81-11 (1981).

2.2. G. A. Carlson, H. L. Barr, B. M. Boghosian, R. S. Devoto, J. N. Doggett, G. W. Hamilton, B. M. Johnston, W. N. Kumai, J. D. Lee, B. G. Logan, R. W. Moir, W. S. Neef, and R. B. Campbell (TRH, Inc.), Comparative End-P lug Study for Tandem Mirror Reactors, LLNL Report, UCID-19271 (1981).

2.3. D. E. Baldwin, B. G. Logan, and T. C. Simonen, Eds., Phys ics Bas is for MFTF-B, LLNL Report, UCID-18496 (1980).

2.4. M. N. Rosenbluth, H. L. Berk, H. V. Wong, D. E. Baldwin, and T. Antonsen, "Fast-Growing Trapped Particle Modes in Tandem Mirrors;" also L. D. Pearlstein, D. E. Baldwin, R. H. Cohen, T. K. FowTer, and B. G. Logan, "Stabilization of Tandem Mirror Trapped Particle Modes by Incomplete Cancellation of Particle Orifts." Both papers presented at the 1982 Sherwood Meeting, Annual Controlled Fusion Theory Conference, Santa Fe, NM, April 25-28, 1982.

2.5. J. W. Van Dam and Y. C. Lee, Proceedings of the Workshop on EBT Ring Physics, Oak Ridge, TN (1979), p. 471.

2.6. G. G. Kelley, "Elimination of Ambipolar Potential-Enhanced Loss in a Magnetic Trap," Plasma Phys, 9, 503 (1967). 



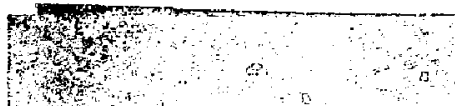

tof

and

$\cdots$

$\therefore$

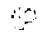

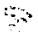

on
25 


\subsection{AXICELL REFERENCE-CASE OPERATION (HARS MODE)}

\subsubsection{DESCRIPTIOH OF REFEREMLE-CASE MARS MODE}

We require a thermal-barrier mode of operation that satisfies the physics and stability constraints mentioned in Sec. 2.1 and that can be scaled to a high-performance reactor. The key features required are the following:

- A high-field circular coll between the central cell and plug to obtain a sufficiently high central-cell density relative to plug density (high $Q$ ).

- A moderately high ( $R \geq 3)$, outward mirror ratio to make thermal barrters and conf ine sloshing ions, wherever the plug is located.

- Trapped-particle stability, obtained by charge separation. This last feature requires a significant number of ions (orders of magnitude above the end-leakage streaming density), which pass from the central cell through the good curvature regions of the yin-yang anchors.

These requirements are met by the following:

- Adding a high-field circular coil at each end of the central cell (see Fig. 2.1-1).

- Increasing the MFTF- 8 yin-yang separation to raise its vacuum mirror ratio from 2 to 3 .

- Putting the thermal-barrier plugs into the extended yin yangs. The high-field circular coil will produce a field of $12 \mathrm{~T}$, using $\mathrm{Hb}_{3} \mathrm{Sn}$ inserts. The thermal-barrier plugs will include sloshing ions, with the ion-confining potential produced by ECRH applied to the outermost sloshing-ion density peaks. These features are included in the axial field, potential, and density profiles shown in Fig. 2.1-2.

To make this scheme adequate for pumping the transition to a desirable low density, axicelis are created by adding another circular coil (but low field) inboard of the high-field circular coils, with beans injected perpendicularly into the axicells to create local density and potential bumps there. The axicells thus serve as electrostatic attenuators, aiding the high-field circular coils in reducing the passing-ion density, relative to the central-cell density, by an additional factor of roughly exp $\left(-\Delta \phi_{p C} / T_{i w C}\right)$. 
Therefore, the anount of collisional trapping and pump-beam power in the transition and barrier regions is reduced. In this role the axicells perform the same function as the density and potential bimps of the yin yangs in the original MFTF-B A-cell design and can be used as experimental "valves" to vary the passing-ion fraction.

Fokker-Planck calculations, recently performed by Archer futch, for cases without axicells and with axicells having equal central-cell densities and 12-tesla mirrors, indicates that axtcells reduce barrier-filling rates by about a factor of 2 to 3 . This reduction leads to an important reduction in pumping in MFTF-B and in reactors. In addition, the passing-ion pressure is reduced in the transitions, easing $W_{0}$ interchange and parallel-current drives there. With respect to traf.ped-particle stability, raising the central-cell density by $\exp \left(\Delta \phi_{p c} / T_{i w c}\right)$ factors allows the use of smaller plasma radius for the same fusion power; the smaller plasma radius in turn increases $\omega^{*} / \gamma_{\text {WHD }}$, thus enhancing drift stabilization of trapped-particle modes.

\subsubsection{SUITABILITY OF REFEREMCE CASE FCR REACTOR OPERATION}

By comparing Figs. 2.1-2 and 2.1-3, one can envision a tanden reactor with fields, potentials, and densities that follow in nearly constant ratios to those of MFTF-B. The reactor fields would be higher everywere by about a factor of two, except for the being four times higher central-cell field. The reactor potentials are all about four times higher (likewise beam voltages), and the reactor densities about ten times higher in the central cell, and five times higher in the transitions and anchors. The plasma radial dimensions, however, would only be slightly larger in the reactor because of high density. Thus the end plug magnets, especially the yin yangs, need only be a little larger than in MFTF-B.

We have already exanined the impact of trapped-particle stability on the mode of operation (e.g.. location of the thermal barriers) in the axice11-magnet configuration and have shown that the same regime of trapped-particle stability ( $\omega^{*}>Y_{M H D}$ for high density, small radius) can be achieved in axicell reactors as well as in the axicell MFTF-B (Table 2.2-1). The following question naturally arises, however: Why use axicells for reactors, rather than A-cel1s or other plug configurations, since reactor suitability is a key motivating issue for MFTF-B. We addressed this 
TABLE 2.2-1. Plasma parameters for reactor types.

\begin{tabular}{|c|c|c|c|c|}
\hline & A-Cell & $\begin{array}{l}\text { Modif ied- } \\
\text { Cusp }\end{array}$ & Axicell & $\begin{array}{c}\text { Axicell } \\
\text { with EBT } \\
\text { Stabilization }\end{array}$ \\
\hline$P_{\text {FUSION }}$ & $3500 \mathrm{MH}$ & 3500 & 3500 & 3500 \\
\hline 0 & 10.3 & 19.7 & 22.3 & 12.2 \\
\hline $\begin{array}{c}\Gamma_{f w} \\
\text { Central cell } \\
\end{array}$ & $2.3 \mathrm{mw} / \mathrm{m}^{2}$ & 2.3 & 2.3 & 2.2 \\
\hline$r_{c}$ & $1.0 \mathrm{~m}$ & 1.0 & 1.0 & 1.0 \\
\hline$r_{c, \text { tnner }}$ & -- & $0.5 \mathrm{~m}$ & -- & -- \\
\hline$r_{f w}$ & $1.3 \mathrm{~m}$ & 1.3 & 1.3 & 1.3 \\
\hline$L_{c}$ & $150 \mathrm{~m}$ & 150 & 150 & 150 \\
\hline$\beta_{c}$ & 0.56 & 0.7 & 0.7 & 0.7 \\
\hline$n_{i c}$ & $1.6 \times 10^{14} \mathrm{~cm}^{-3}$ & $2.2 \times 11^{14}$ & $1.6 \times 10^{14}$ & $1.6 \times 10^{14}$ \\
\hline$T_{i c}$ & $40 \mathrm{keV}$ & 40 & 40 & 40 \\
\hline$T_{e c}$ & $36 \mathrm{keV}$ & 33 & 32 & 33 \\
\hline $\begin{array}{c}\phi_{e} \\
\text { Barrier/Plug }\end{array}$ & $270 \mathrm{keV}$ & 260 & 240 & 240 \\
\hline$L_{B}$ & $8 \pi$ & 7 & 8.6 & 8.6 \\
\hline$B_{B}$ & 0.63 & 0.61 & 0.71 & 0.36 \\
\hline$n_{\text {pass }}$ & $5.0 \times 10^{12} \mathrm{~cm}^{-3}$ & $5.5 \times 10^{12}$ & $3.4 \times 10^{12}$ & $4.4 \times 10^{12}$ \\
\hline$E_{i n j, B}$ & $350 \mathrm{keV}$ & 300 & 250 & 250 \\
\hline$E_{\text {eh }}$ & $520 \mathrm{keV}$ & 390 & 750 & 240 \\
\hline$T_{\text {ew }}$ & $93 \mathrm{keV}$ & 110 & 82 & 150 \\
\hline$\phi_{B}$ & $230 \mathrm{keV}$ & 190 & 210 & 370 \\
\hline $\begin{array}{c}\phi_{C} \\
\text { Anchor }\end{array}$ & $150 \mathrm{keV}$ & 150 & 140 & 140 \\
\hline$r_{A}$ & $0.84 \mathrm{~m}$ & & 1.2 & \\
\hline$B_{A}$ & 0.7 & & 03 & \\
\hline$n_{i A}^{n}$ & $1.7 \times 10^{14} \mathrm{~cm}^{-3}$ & & $1.5 \times 10^{13}$ & \\
\hline$E_{i n j, A}$ & $200 \mathrm{keV}$ & & 150 & \\
\hline$E_{i A}$ & $310 \mathrm{keV}$ & & 150 & \\
\hline$T_{e A}$ & -- & & $32 \mathrm{keV}$ & \\
\hline$\phi_{A}$ & -- & & $160 \mathrm{keV}$ & \\
\hline$\phi_{p c}$ & $19 \mathrm{keV}$ & & -- & \\
\hline
\end{tabular}


question in a recently completed comparative study (Ref. $2.2 \mathrm{Sec} .2 .1$ ) at LLML of four plug configurations: A-cell (fig. 2.2-1), axicel1 (Fig. 2.2-2), modified-cusp (Fig. 2.2-3), and hot-electron-ring (EBT) stabilized axicel1 (Fig. 2.2-2., but without the yin-yang anchor). While these four configurations do not constitute an exhaustive list of possible plug configurations, their physics characteristics are probably generic to the three known means of providing minimum-B for HHD stability in open systems: quadrupoles, cusps, and damagnetic wells created by fast-drifting electrons. The basic results of that study are sumarized in Tables 2.2-2 to 2.2-4. These tables show significantly lower plug-magnet cost and higher $Q$ (less plug beans and ECRH) for the axicell and modified-cusp plugs compared to the A-cell design. The axicell with EBT stabilization had the smallest plug-magnet cost, but the additional ECRH required to maintain the EBT rings still gave it a higher overall capital cost per kilowatt. The main reason for the lower plug-magnet cost and higher $Q$ of both axicell (with yin-yang anchors) and modified-cusp plugs (compared to the A-cell) was that higher fields could be designed in the circular mirror coils and could be augmented by normal copper coits to permit fields even above superconducting limits with modest electric-power consumption. The high mirror fields, together with barrier pumping, increased the allowed ratio of central-cell density to plug density, thus increasing $Q$ and lowering other plug magnetic fields and costs. An additional advantage of the circular mirror coils can be expected in the reduction of radial transport in the central cell (although credit for this was not taken in the study comparisons). Although the magnet cost and $Q$ of the modified-cusp was comparable to the axicell, the axice 11 with quadrupole anchors was chosen as the favored-candidate reactor configuration for the ongoing Mirror-Advanced-Reactor Study (MARS). This was primarily because of greater confidence in the MHO stability of quadrupoles employed in many past and present mirror experiments and because of the forthcoming data base that will be provided by TMX-UI at LLML, TARA at MIT, Gama-10 in Japan, and AMBAL-1 in the USSR.

Since the comparative study, the MARS axicell-reactor design has been further developed in four important areas:

(1) Preliminary engineering analyses by General Dynamics, LLNL, and the University of Wisconsin on the neutron damage, water cooling, power consumption, and stress-analys is of the normal coil inserts 


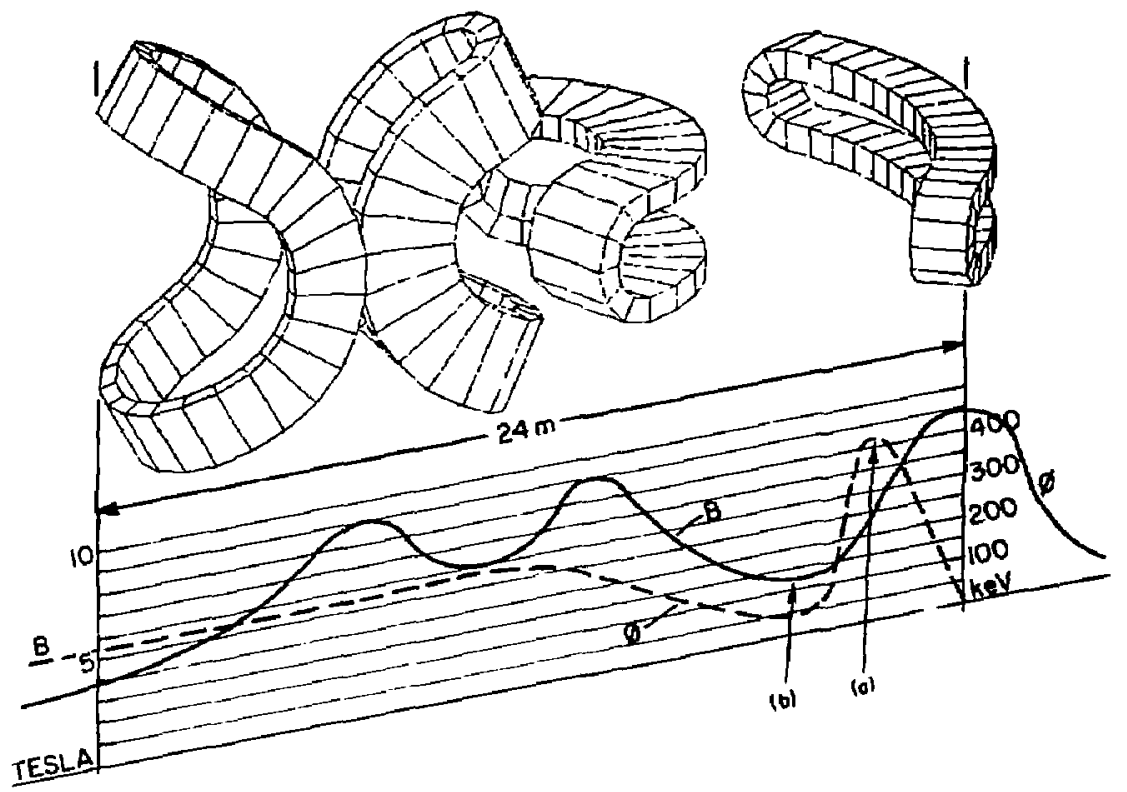

Fig. 2.2-1. A-cell Tandem-Mirror Reactor (TMR). 


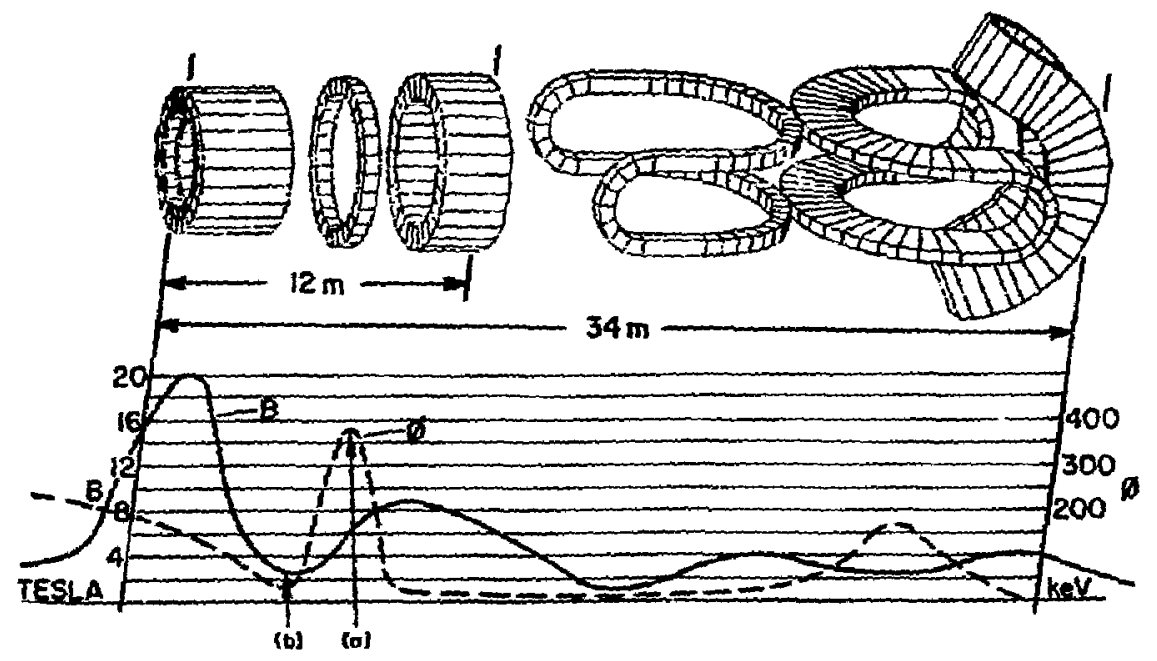

Fig. 2.2-2. Axicell TMR. 


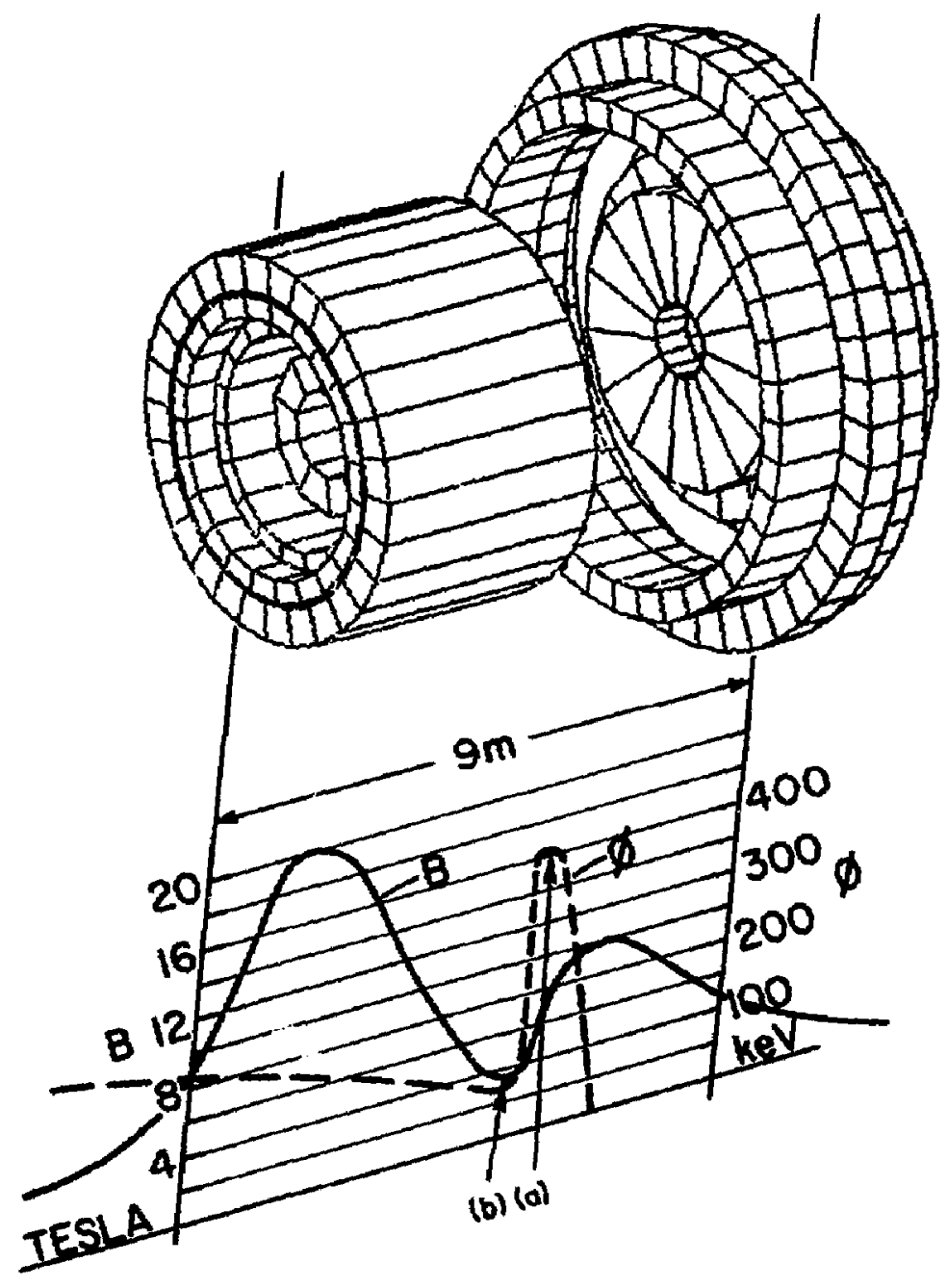

Fig. 2.2-3. Modified cusp TMR. 
TABLE 2.2-2. Reactor power balance (all powers in megawatts).

\begin{tabular}{|c|c|c|c|c|}
\hline & A-Cell & $\begin{array}{l}\text { Modif ied- } \\
\text { cusp }\end{array}$ & Axicell & $\begin{array}{c}\text { Axicell } \\
\text { with EBT } \\
\text { stabilization }\end{array}$ \\
\hline \multicolumn{5}{|l|}{ Trapped powers: } \\
\hline ECRH & 97 & 60 & 47 & 154 \\
\hline Pump beams & 148 & 98 & 93 & 97 \\
\hline Sloshing beams & 66 & 20 & 12 & 35 \\
\hline Anchor beams & 29 & $-\infty$ & 5 & -- \\
\hline TOTAL & 340 & 178 & 157 & 286 \\
\hline \multicolumn{5}{|l|}{ Injected powers: } \\
\hline ECRH & 108 & 67 & 52 & 171 \\
\hline Pump beans & 177 & 118 & 115 & 105 \\
\hline Sloshing beams & 122 & 83 & 52 & 159 \\
\hline Anchor beans & 30 & -- & 15 & -- \\
\hline TOTAL & 437 & 268 & 234 & 435 \\
\hline \multicolumn{5}{|l|}{ Recirculated powers: } \\
\hline ECRH & 216 & 134 & 104 & 342 \\
\hline Pump beams & 354 & 236 & 230 & 210 \\
\hline Sloshing beams & 244 & 166 & 104 & 318 \\
\hline Anchor beams & 60 & -- & 30 & $\cdots$ \\
\hline Copper coils & -- & 72 & 72 & 72 \\
\hline TOTAL & 874 & 608 & 540 & 942 \\
\hline Thermal converter output & 1176 & 1176 & 1176 & 1176 \\
\hline Direct converter output & 520 & 439 & 429 & 493 \\
\hline TOTAL (Gross electrical) & 1) 1696 & 1615 & 1605 & 1669 \\
\hline$f_{\text {REC }}=\frac{\text { Recirculated power }}{\text { Gross electrical }}$ & 0.52 & 0.38 & 0.34 & 0.56 \\
\hline Net electrical power & 822 & 1007 & 1065 & 727 \\
\hline
\end{tabular}


TABLE 2.2-3. Reactor costs in millions of dollars (\$M).

\begin{tabular}{|c|c|c|c|c|}
\hline & A-Cell & $\begin{array}{l}\text { Modified- } \\
\text { Cusp }\end{array}$ & Axicell & $\begin{array}{c}\text { Axicell } \\
\text { with EBT } \\
\text { Stabilization }\end{array}$ \\
\hline Beams $0 \$ 2 / W$ injected & 658 & 402 & 364 & 528 \\
\hline ECRH e\$5/W injected & 540 & 335 & 260 & 855 \\
\hline Plug magnets & 628 & 251 & 302 & 175 \\
\hline Direct converter & 158 & 83 & 152 & 127 \\
\hline Central cell & 450 & 450 & 450 & 450 \\
\hline Reactor bldg. & 165 & 154 & 162 & 149 \\
\hline BOP & 470 & 470 & $4 \pi 0$ & 470 \\
\hline TOTAL & 3069 & 2145 & 2160 & 2754 \\
\hline
\end{tabular}

TABLE 2.2-4. Reactor costs in dollars per tilowatt-electric ( $\$ / \mathrm{kHe}$ ).

\begin{tabular}{|c|c|c|c|c|}
\hline & A-Cell & $\begin{array}{l}\text { Modified- } \\
\text { Cusp }\end{array}$ & Axicell & $\begin{array}{c}\text { Axicell } \\
\text { with EBT } \\
\text { Stabilization }\end{array}$ \\
\hline Beams $92 / \mathrm{W}$ injected & 800 & 399 & 342 & 639 \\
\hline ECRH $0 \$ 5 / \mathrm{W}$ injected & 657 & 333 & 244 & 1176 \\
\hline Plug magnets & 764 & 249 & 284 & 212 \\
\hline Direct converter & 192 & 82 & 143 & 152 \\
\hline Central cell & 547 & 447 & 423 & 545 \\
\hline Reactor bidg. & 201 & 153 & 152 & 180 \\
\hline BOP & 572 & 467 & 441 & 569 \\
\hline TOTAL & 3733 & 2130 & 2029 & 3473 \\
\hline
\end{tabular}


TABLE 2.2-5. Key parameters of axicel1 tandem reactors.

\begin{tabular}{|c|c|c|c|}
\hline & $\begin{array}{l}\text { FY81 Stuóy UCID } 19271 \\
\text { (TARA Mode) } \\
\text { (no thermal alphas) }\end{array}$ & $\begin{array}{l}\text { Higher Field Axicell } \\
\text { (TARA Mode) } \\
\text { (with thermal alphas) }\end{array}$ & $\begin{array}{l}1982 \text { MaRs Study } \\
\text { (New Operating Mode) } \\
\text { (with thermal alphas) }\end{array}$ \\
\hline PFUSIOH & $3500 \mathrm{NH}$ & 3500 & 3500 \\
\hline$L_{c}$ & $150 \mathrm{~m}$ & 150 & 150 \\
\hline Lbarrier & $8.6 \mathrm{~m}$ & 8.0 & 7.0 \\
\hline $\mathbf{B}_{\mathbf{C}}$ & $3.0 \mathrm{~T}$ & 4.7 & 4.7 \\
\hline Binirror & $20 \mathrm{~T}$ & 24 & 24 \\
\hline$r_{c}$ & $1.0 \mathrm{~m}$ & 0.38 & 0.38 \\
\hline$r_{F W}$ & $1.3 \mathrm{~m}$ & 0.56 & 0.56 \\
\hline $\mathbf{n}_{\mathbf{i c}}$ & $1.6 \times 10^{14} \mathrm{~cm}^{-3}$ & $4.6 \times 10^{14}$ & $4.6 \times 10^{14}$ \\
\hline$n_{\text {pass }}$ & $3.4 \times 10^{12} \mathrm{~cm}^{-3}$ & $1.0 \times 10^{13}$ & $3.6 \times 10^{12}$ \\
\hline$Q$ & 22.3 & 76.8 & 17.0 \\
\hline$\Gamma_{\mathbf{F W}}$ & $2.3 \mathrm{MH} / \mathrm{m}^{2}$ & 5.3 & 5.3 \\
\hline
\end{tabular}


indicates that high field mirror coils as high as $28 \mathrm{~T}$ are probably feasible, with the cost-effectiveness optimum being about $24 \mathrm{~T}$.

(2) Blanket modules capable of operating at $5 \mathrm{mw} / \mathrm{m}^{2}$ of neutron wall loading permit us to raise the central-cell field and density, shrinking the plasma size down to the point where the yin-yang anchor need be only slightly larger than the MFTF-B coil, using NbTi conductor.

(3) Equilibrium concentrations of thermalized alphas were included in the plasma mode 1 (resulting in reduced $Q$ by nearly a third) with provision being made for $d c$ grad-B radial transport (akin to an optimized resonant transport in the central cell). On the plus side, the ignited central cell now provides 50 to $100 \mathrm{MW}$ of power to sustain a halo dense enough to shield the plasma core from gas and impurities and provides a handle on reducing the central-cell radial electric field and associated $E \times B$ rotation.

(4) Conversion of the axicell-plug operating mode, from the TARA-like mode of the previous year to the more stable regime (with respect to trapped-particle instabilities) of thermal-barrier operation in the yin yang, has been successfully completed. Minimal perturbation occurred with respect to the central-cell design and plug magnets (with the single exception being the transition coil design, which is still being optimized to null parallel current). For comparison, key parameters of the FY81 axicell design [the first (TARA-mode) MARS design] and the recently modified axicell MARS design are given in Table 2.2-5. Note that conversion to the modified axicell MARS design made very little difference in the performance. The drop in Q from FY8I axicell to the MARS design was a consequence of properly including thermal alphas in the plasma model and power balance.

\subsubsection{OPERATING SCENARIO FOR MARS MODE AT STEAOY STATE}

We now examine the plasma parameters and power balances for the MARS mode, using plasma models described in detail in Sec. 2.4, and will shortly consider the stability aspects of this case. Parameters for the central-cell, axicell, transition, and anchor plugs (Table 2.2-6) are consistent with the following key engineering choices for MFTF-B: 
TABLE 2.2-6. Plasma parameters for the MARS mode of the MFTF-B Axice11.

\begin{tabular}{|c|c|c|}
\hline Region & Paraneter & Value \\
\hline \multirow[t]{10}{*}{ Central-cell } & $T_{\text {iwC }}$ & $15 \mathrm{keV}$ \\
\hline & $\mathbf{T}_{\mathrm{eC}}$ & $9 \mathrm{keV}$ \\
\hline & $n_{\text {iwe }}$ & $4.8 \times 10^{13} \mathrm{~cm}^{-3}$ \\
\hline & $B_{C}$ & 0.5 \\
\hline & $\phi c$ & $30 \mathrm{kV}$ \\
\hline & $\phi$ & $50 \mathrm{kV}$ \\
\hline & $(n \tau)_{\text {Pastukhov }}$ & $5 \times 10^{13} \mathrm{~cm}^{-3} \mathrm{~s}$ \\
\hline & $Q_{C}$ & 0.6 \\
\hline & $\mathbf{Q}_{\text {tot }}$ & 0.4 \\
\hline & $\mathbf{L}_{C}$ & $16.5 \mathrm{~m}$ \\
\hline \multirow[t]{8}{*}{ Yin-yang } & $n_{i A}(b)$ & $3.4 \times 10^{12} \mathrm{~cm}^{-3}$ \\
\hline & $\phi$ & $51 \mathrm{kV}$ \\
\hline & $E_{\text {eh }}$ & $470 \mathrm{keV}$ \\
\hline & $B_{A}$ & 0.55 \\
\hline & $\phi$ & $80 \mathrm{kV}$ \\
\hline & $T_{\text {ow }}$ & $72 \mathrm{keV}$ \\
\hline & $\delta \phi_{a}$ & $72 \mathrm{kV}$ \\
\hline & $n_{\text {ihA }}(a)$ & $5.6 \times 10^{12} \mathrm{~cm}^{-3}$ \\
\hline \multirow[t]{4}{*}{ Transition } & $\phi_{T}$ & $26 \mathrm{kV}$ \\
\hline & $n_{T}$ & $2 \times 10^{12} \mathrm{~cm}^{-3}$ \\
\hline & $B_{T}$ & 0.05 (0.01 perpendicular, \\
\hline & & and 0.09 parallel) \\
\hline \multirow[t]{4}{*}{ Axicell } & $n_{i x}$ & $1.2 \times 10^{14}$ \\
\hline & $\Delta \phi_{p c}$ & $9.0 \mathrm{kV}$ \\
\hline & $\Delta \phi_{\mathrm{pb}}$ & $10 \mathrm{kV}$ \\
\hline & $B_{X}$ & 0.20 \\
\hline
\end{tabular}


TABLE 2.2-6 (continued)

Trapped current and rf power:

Neutral Beams

Each axicell $(80 \mathrm{kV})$

Trapped current (A)

Each transition ( $30 \mathrm{kV}$ )

3.4

Each anchor $(80 \mathrm{kV})$

6.0

Each pump beam ( $80 \mathrm{kV}$ )

0.30

2.4

ECRH

Absorbed power (kW)

Anchor $(28,35,56 \mathrm{GHz})$

670

ICRH

Central cell (15-17 MHz)

210 
- 12-T maximum high-field circular coils (1imited by the superconducting $\mathrm{Nb}_{3} \mathrm{Sn}$ insert).

- $\quad$ An $R_{y a c}=3$ yin yang with $B_{\text {mirror }}=3 T(2$ the maximum separation manageable with the MFTF-B structure).

- An 80-kV max, 30-s beam with 90\% atomic-species fraction, and 28-, 35-, and 56-6Hz gyrotrons.

The parameters in Table 2.2-6 are also consistent with the performance levels of the previous A-cell design, namely: $(n \tau)_{C} \approx 5 \times 10^{13} \mathrm{~cm}^{-3} \mathrm{~s}$, $T_{i c}=15 \mathrm{keV}, T_{e c}=9 \mathrm{keV}, \phi_{c}=30 \mathrm{keV}, Q \sim 0.5$ and $\left.P_{\text {fusion }}{ }^{2}\right) \mathrm{MW}$ (equivalent for $D-T$ ). Using definitions in the previous A-cell proposal (Refs. 2 and 3 of Sec. 2.11, we find:

$$
q_{c} \equiv \frac{P_{\text {fusion }}}{T_{\text {pastukhov loss }}\left(\phi_{c}+\phi_{e}+T_{i c}+T_{e c}\right)}=0.6,
$$

and $Q_{\text {overa } 11} \equiv \frac{P_{\text {fusion }}}{\sum \begin{array}{l}\text { aTTbeams, ECRH and ICRH } \\ \text { trapped in the plasma }\end{array}}=0.4$,

for this axicell MARS mode of operation of MFTF-B. The fusion power $P_{\text {fusion }} \approx 640 \mathrm{~kW}$ from the central cell and $190 \mathrm{~kW}$ from the two dense axicells. The fact that similar Q's and fusion power obtain for this case in spite oi shorter central-cell length $\left(L_{c}\right.$ (eff) $=16.5 \mathrm{~m}$ wersus $32 \mathrm{~m}$ for the A-cell design) and smaller central-cell radius $\left(r_{c} \approx 0.3 \mathrm{~m}\right.$, max allowed by $12-T$ insert, versus $r_{c}=0.56 \mathrm{~m}$ for the A-cell design) is due to higher central-cell density $\left(n_{c}=4.8 \times 10^{73} \mathrm{~cm}^{-3}\right.$ versus $2 \times 10^{13} \mathrm{~cm}^{-3}$ in the A-cell design). The higher central-cell density is made possible by the higher 12-T circular coil compared to the previous 4-T yin-yang mirror kthich played the same role. Mapping of densities in thermal-barrier machines leads one to expect higher central-cell/plug density ratios in proportion to the maximum mirror field. However, the central-cell density is not exactly three times higher than in the A-cell design because of the effect of the outer potential drop $\Delta \phi_{\mathrm{pb}}$ in the axicell (see Fig. 2.1-2) which accelerates the passing ions through the high-field mirror (see Eq. 14 in Sec. 2.4 for details).

Figures 2.2-4 to 2.2-6 illustrate schematically the central-cell ion-particle flows, central-cell ion-power balance, and central-cell electron-power balance, respectively, for the MARS-mode of MFTF-B. These 
Charge exchange loss on HEPB

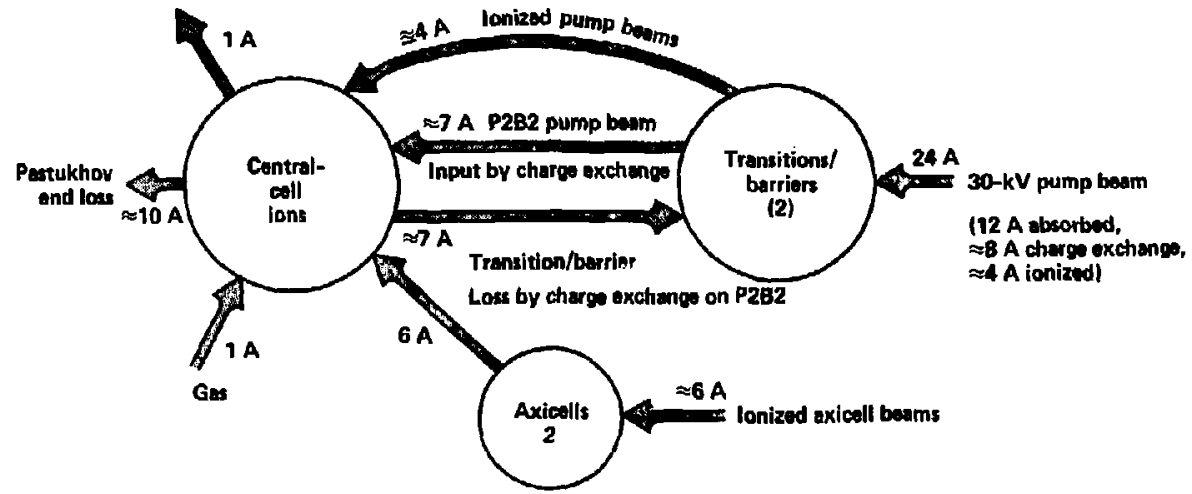

Fig. 2.2-4. Typical centra1-cell particle balance for MARS mode of MFTF-B. 


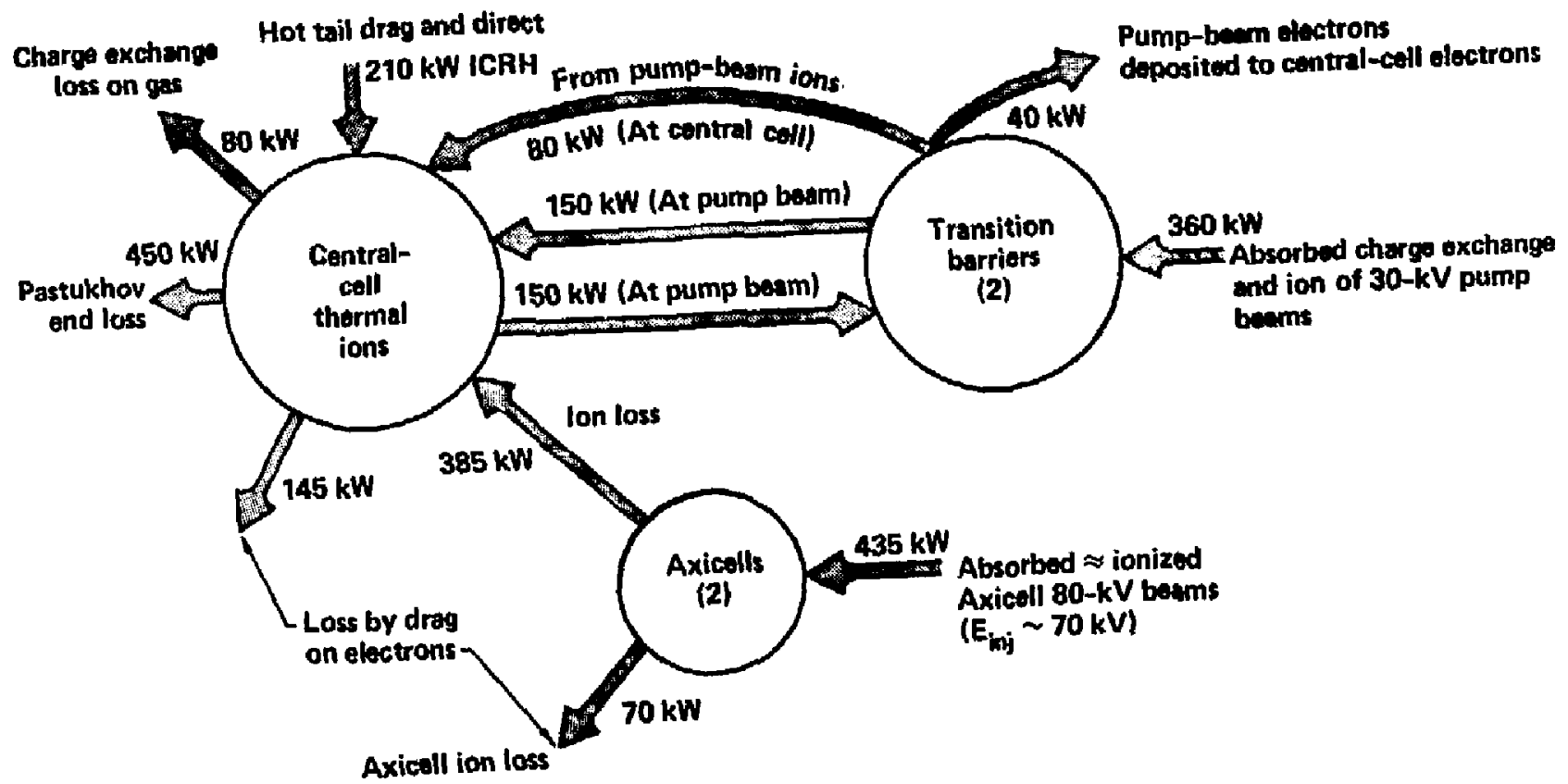

Fig. 2.2-5. Typical central-cell ion-energy balance for MARS mode of MFTF-B. 


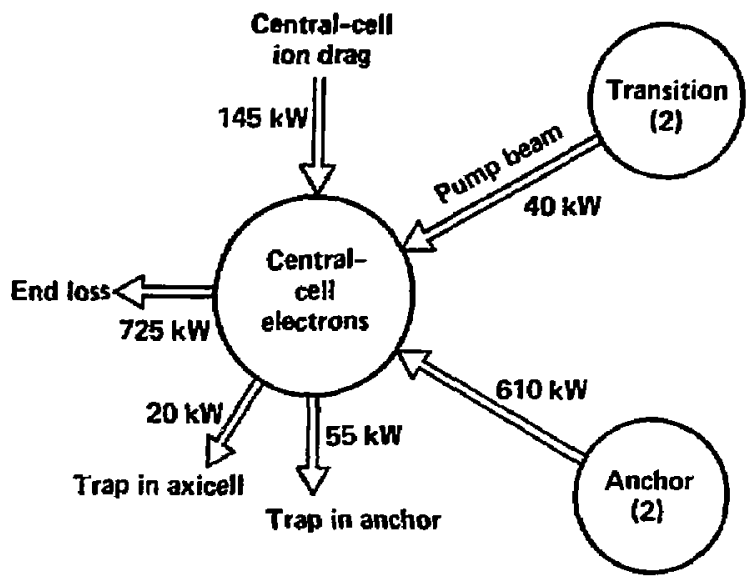

Fig. 2.2-6. Typical central-celi electron-energy balance for MARS mode of MFTF-B. 
values correspond to the parameters in Table 2.2-6. Essentially, Fig. 2.2-4 shows that the central cell is fueled by the axicell beams (since the lowest axicell mirror is towards the central cell) and by the 30-kV pump beams of the transition regions, which have enough energy to pass deposited ions over the axicell-potential hill and into the central cell but not over the $\phi_{c}$ potential peaks at the extreme ends of the plugs. . ne axicell-beam power can be varied by a factor of at least two to control the amount of central-cell fueling and heating.

Figure 2.2-5 shows that the central-cell-ion power input from the axicells must ie supplemented by ICRH, because an $80-k V$ beam-voltage limit does not provide enough energy per ion lost.

Figure: 2-6 shows a simplified electron-power balance (leaving out many of the details) that involve ine major parts of the power flow. Input power to the central-cell electron is dominated by the ECRH in the anchors. Hot and warm ECRH electrons in the plugs/anchors originate from the passing central cell electrons reaching the ECRH resonance 2ones. Furthermore, the electron-confining potential is maximum at the outermost yin-yang mirror. Therefore, scattering and drag losses of these heated electrons, for the most part, ends up rethermalizing back into either the transition or central cell by conduction and convection.

\section{Stability Considerations}

Having described the reference-case MARS-mode of MFTF-B, which is consistent wi h power balance and engineering constraints, we now show it is reasonably consistent with nominal theoretical criteria for plug microstability, trapped-particle stability, and $M H D$ stability for interchange beta limits and MHD-response of hot electrons. Indeed the stability criteria described by jaldwin (Sec. 1 of this report) were used to guide the evolution of this design. We are still in the process of solving MHO stability, including ballooning at low mode numbers, with finite Larmor radius (FLR) and conditions for null parallel current in the transitions and anchors. However, we believe we still have flexibility to lower the beta in the axicell to 10 to 15\%, if needed for ballooning (by lowering axicell beam voltage somewhat and raising ICRH power in the central cell), and to further modify the transition-coil design, if needed to reduce the equilibrium parallel currents in the ends. 
With respect to plug/anchor microstability, we note that this design for MFTF-B, which puts the thermal barrier in the yin yang with a larger mirror ratio, is very similar to the TMX-U, which is designed stabie with respect to present theory for drift-cyclotron-ioss-cone (DCLC), axial-loss-cone, arid Alfuén-ion-cyclotron modes. Data from testing these predictions will be available in $t$ ime to guide the location of beams and ECRH in the MFTF- $B$ yin yangs. For the reference-case thermal-barrier MARS mode of MFTF-B, the microstability analysis of Appendix $F$ in this report indicates that a midplane warm-ion fraction, $n_{\text {warm }} / n_{\text {hot }}=0.75$ at $T_{\text {warm }} / T_{\text {hot }}>0.3$, should be nominally stable. This is consistent with the parameters in Table 2.2-6: $n_{\text {warm }} / n_{\text {hot }}=g_{b} n_{\text {pass }}\left(B_{A} j / n_{s i o s h}\left\langle B_{A}\right)=0.8\right.$, and $T_{\text {warm }} / T_{\text {hot }}=T_{i w C} /\left(\frac{2}{3} E_{i n j}^{S l o s h}\right)=0.32$. As noted in Appendix $F$, the stability boundary varies with the hot-electron axial profile, which can in principle be controlled by tine location of the ECRH resonances. Also, to add flexibility, the sloshing-ion density can be decreased some $20 \%$ with respect to the passing-ion density.

The stability criteria of Baldwin for conversion of trapped-particle modes to stable drift waves by charge separation in the anchors (see Eq. 1.2 in Sec. 1) can be written for the $m=1$ mode as:

$$
\omega_{* j}^{2}>4 \gamma_{M H D}^{2}\left(1+A_{r}\right) / A_{r}^{2},
$$

where

$$
A_{r} \equiv\left(\frac{r_{c}}{\rho_{i c}}\right)^{2}\left(\frac{B_{c}}{B_{a}}\right)\left(\frac{\overline{g_{b} n_{p a s s}}}{n_{c}}\right)\left[\frac{2 L_{A}(e f f)}{L_{c}}\right]
$$

is the Rosenbluth parameter; $g_{b} n_{\text {pass }}$ is the effective passing-ion density into the anchor minus the jassing-electron density, averaged jver the good curvature regions of the anchors of effective length $L_{A}$ (eff). The effective length depends in part on the assumed shape of the trapped-particle mode $\phi(z)$, which has not yet been precisely determined, so therefore $L_{A}$ (eff) and $g_{b} n_{p a s s}$ cannot be precisely determined.

Figure 2.2-7 indicates that $g_{b} n_{\text {pass }}$ would be about $2 \times 10^{12} \mathrm{~cm}^{-3}$ in the MFTF-B yin yang, over a characteristic length of about $2.5 \mathrm{~m}$, if the worst trapped-particle eigen mode rolls off just where the pressure-weighted good 


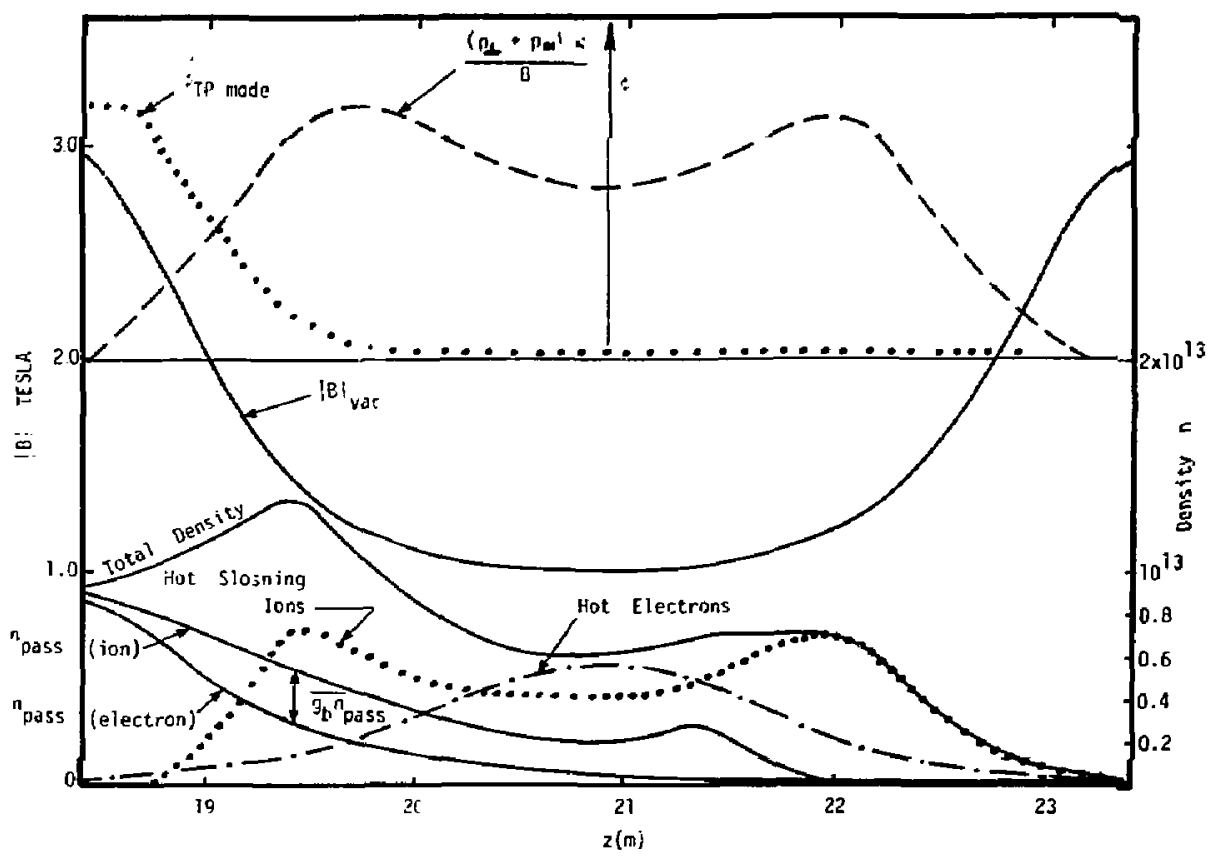

Fig. 2.2-7. Comparison of passing ions and electrons in MFTF-B yin-yang anchor. 
curvature in the yin yang begins to rise. Since the trapped cold ions of density $\left(g_{b}-1\right) n_{\text {pass }}$ turn well beyond the mode's roll off (in fact way back near the 12-T high-field mirror) they also contribute to the charge separation [although somewhat $(\sim 30 \%)$ less effectively than strict]y passing ions from the central cel1] for yin-yang transition lengths only a few times longer than $L_{A}$ (eff) (according to Baldwin's analysis using a coupled, three-square-well region mode]).

The actual effective $g_{b}$ to use in Eqs. 2.2.3 and 2.2.2 is uncertain to the extent it still depends on the actual "worst" eigenmode shape. Given these uncertainties, we cannot make firm statements now regarding the "margin of safety" for the particular reference case we have chosen for MFTF-B. However, we will shortly indicate how we can vary the operating parameters of MFTF -B to accommodate, up to a factor of four or so, a "worse"-stability boundary than that represented by Eq. 2.2.1. In Eq. 2.2.1, $Y_{M H D}$ is the average MHD growth rate (without anchors), averaged over all the bad MHD drives of the centra] cell, axicell, and yin-yang transition regions. Since all three regions have comparable drives at present, but the axicell and transition drives might be reduced relative to the center cel] by future design optimization, we can characterize $\gamma_{M H D}$ in terms of the irreducible central-cell drive $\propto R_{C}^{-1} \propto r_{c} / L_{\text {tran }}^{2}(c c)$. Hence

$$
r_{M H B}^{2} \equiv 0 r_{c c}^{2} \approx 0 \frac{2 T_{i c}\left(\frac{r_{c}}{p_{c}}\right)\left(\frac{d p_{c}}{d r}\right) L_{t r a n}(c c)}{M_{i} r_{c} R_{c} L_{c}},
$$

where the proportionality constant $D$ depends on the aggregate bad-curvature regions (being unity in the limiting case of central-cell drive only) and where $L_{\text {tran }}(c c)$ is the characteristic central-cell (axisymmetric) transition length. Then using

$$
w_{i}^{t}=\frac{T_{j c}\left(\frac{r_{c}}{p_{c}}\right)\left(\frac{d p_{c}}{d r}\right)}{q E_{c} r_{c}^{2}} \text {, }
$$

where $\left(r_{c} / p_{c}\right)\left(d p_{c} / d r\right)$ is the pressure-gradient length normalized to central-cell radius, Eq. 2.2.1 can be solved to estimate the required passing density ( $t$ imes the effective $g_{b}$ ): 


$$
\frac{\overline{g_{b} n_{p \text { ass }}}}{n_{c}}>\frac{8 D}{\left(\frac{c}{p_{c}}\right)\left(\frac{{ }^{r}}{d r}\right)} \quad\left(\frac{r_{c}^{2}}{L_{\operatorname{tran}}(c c) L_{A}(\text { eff })}\right)\left(\frac{{ }_{A}}{B_{c}}\right) .
$$

The interesting feature exhibited by $E q .2 .2 .5$ is that the required passing-ion-density fraction for trapped-particle stability actually decreases with $p$ lasma radius $r_{c}$, in spite of the fact that $A_{r} \propto r_{c}^{2} / \rho_{i c}^{2}$ would be reduced. This is because $\omega_{*}^{2} \sim 1 / r_{c}^{4}$ overpowers the $r_{c}^{2}$ dependence of $A_{r}$. Thus smaller radius plasmas, relative to axial scale lengths, allow smaller $g_{b} n_{\text {pass }} / n_{c}$ and hence higher tandem-mirror $Q$ :

$$
Q \propto \frac{P_{\text {fusion }} \text { (central cell) }}{P_{\text {injection }}(p \text { tugs) }} \propto\left(\frac{n_{c}}{g_{b} n_{\text {pass }}}\right)^{2} .
$$

One caution is that $r_{c}$ cannot be made too small relative to $\rho_{i c}$ or else other instabilities such as low hybrid drift waves might become dominant.

Table 2.2-7 presents key stability-related parameters for MFTF-B and also for reactors to show that the high $\omega_{\star} / Y_{M H O}>1$ regime for MFTF-B can also be utilized in reactors. Note in Table 2.2-7 that $r_{c} / \rho_{i c} \sim 13$ in MFTF-B, while $r_{c} / \rho_{i c}{ }^{2} 40$ still allows $\omega_{k}>\gamma_{M H D}$ in reactors (because $\gamma_{M H D}$ is small in long, thin aspect-ratio plasmas).

Some flexibility exists in the MFTF-B operation to accomodate a larger passing-ion fraction than is estimated by Eq. 2.2.5, by means other than simply reducing the plasma radius further (which would begin to limit $\left.r_{c} / \rho_{\text {ic }}<10\right)$. In the event $n_{p a s s} / n_{c}$ needs to be increased by more than a factor of two, it would have to be done in part by reducing $n_{c}$, since $n_{p a s s}$ could not be increased more than twice in the anchor without exceeding ayailable ECRH power or, just beyond that limit, exceeding the cutoff density for microwave penetration. Thus, $n_{c}$ would be limited beyond that which could otherwise be supported by the combination of axicell-potential bump and the 12-T mirror. The limiting case in which the axicell beam and the transition pump beam are turned off, allowing the transition to fill to a Maxwellian ion distribution, is the TMX-U mode described in Sec. 2.3.2 (Fig. 2.3-3). By varying the amounts of transition pump beams and axicell beams, one could vary $n_{c}$ over a range of four while keeping the anchor/plug parameters essentially constant (see Fig. 2.2-8). If the central-cell ICRH 
TABLE 2.2-7. Trapped-particle stability parameters for MFTF-B and for axicell tandem-mirror reactors.

\begin{tabular}{|c|c|c|c|}
\hline Parameter & $\begin{array}{l}\text { MFTF-B } \\
\text { (reference case } \\
\text { thermal barriers) }\end{array}$ & $\begin{array}{l}\text { Mirror-Advanced } \\
\text { Reactor Study } \\
\text { (MARS) } \\
\text { (candidate) }\end{array}$ & $\begin{array}{l}\text { Original Axicell Reactor } \\
\text { (FYB1 report UC1D 192J1) }\end{array}$ \\
\hline$r_{C}(m)$ & 0.3 & 0.4 & 1.0 \\
\hline $\mathrm{T}_{\text {jwC }}(\mathrm{keV})$ & 15 & 35 & 40 \\
\hline$B_{C}(T)$ & 1 & 4.7 & 3.0 \\
\hline$r_{c} / \rho_{i c}$ & 13 & 40 & 60 \\
\hline $\mathrm{nc}\left(\mathrm{cm}^{-3}\right)$ & $4.5 \times 10^{13}$ & $4.5 \times 10^{14}$ & $1.6 \times 10^{14}$ \\
\hline$B_{A}(T)$ & 1 & 2 & 1.4 \\
\hline$g_{b} n_{p a s s}\left(B_{A}\right)$ & $2 \times 10^{12}$ & $1 \times 10^{13}$ & $21 \times 10^{11}$ \\
\hline $\begin{array}{l}\frac{r}{p} \frac{d p}{d r} \\
\text { (gradient parameter) }\end{array}$ & 2.8 & 3.0 & 3.0 \\
\hline$D \equiv \gamma_{M H D} / \gamma_{C C}$ & 3 & 6 & 9 \\
\hline$\omega_{* j} / Y M H D$ & 1.6 & 1.0 & 0.21 \\
\hline$L_{\operatorname{tran}}(c c)(m)$ & 5 & 12 & 12 \\
\hline$L_{A}($ eff $)(m)$ & 2.5 & 4 & 3 \\
\hline$L_{c}(e f f)(m)$ & 16.5 & 150 & 150 \\
\hline $\begin{array}{l}A_{r} \\
\text { (Rosenbluth Parameter }\end{array}$ & 2.3 & 4.5 & 0.2 \\
\hline$g_{b} n_{p a s s} / n_{c}$ & $4 \times 10^{-2}$ & $2.2 \times 10^{-2}$ & $\begin{array}{c}6.3 \times 10^{-4} \text { ys } \\
.3 \text { required for stability }\end{array}$ \\
\hline
\end{tabular}




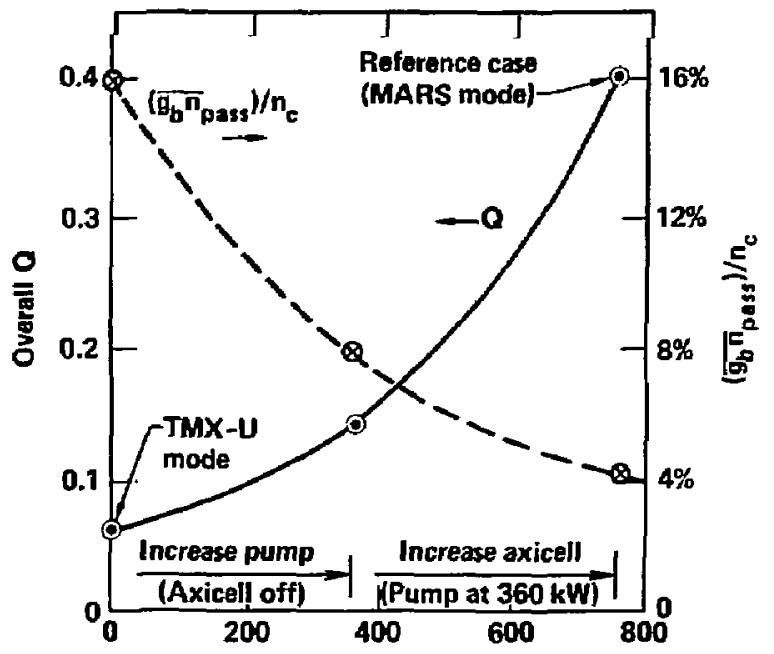

Axicell + transition pump-beam power (kW)

Fig. 2.2-8. Variation of overall $Q$ and of ion fraction passing into the yin-yang anchor as a function of axicell- and pump-beam power. 
heating is also varied appropriately, $T_{i c}$ and $(n \tau)_{c}$ could also be kept nearly constant.

We turn now to KHD-interchange stability. Figure 2.2-9 shows an example of a flute interchange calculation using TEBASCO (see Appendix B) for the vacuum magnetic fields of the coil set of Fig. 2.2-4, with pressure models appropriate for the reference-case thermal barrier (MARS) mode of MFTF-B operation. Although the flute beta limits would change with finite beta fields and for coils readjusted to null parallel current, Fig. 2.2-9 indicates that overall flute interchange stability is roughly consistent with the betas required in the reference case (see Table 2.2-6). These betas $\left(\beta_{C}=0.5\right.$ in the central cell, $\beta_{X}=0.2$ in the axicell, and $\left.\beta_{T}=0.05\right)$ give rise to comparable contributions to bad curvature drive (weighted with pressure) from each of those three regions, as can be seen in Fig. 2.2-9. Thus, MHD-interchange stability requires a nominal anchor beta $B_{A}=0.5$. In order for this anchor beta to count as good MHD pressure (as assumed in the flute interchange calculation of Fig. 2.2-9), the local midplane sloshing-ion beta must well exceed the Lee-Van Dam criterion so that the hot electrons, which make up most of the $B_{A}=0.5$, will have an MHD response:

$$
B_{\text {slosh }} \gg \frac{2 r_{n}}{R_{A}} \text {. }
$$

For the reference case, $\beta_{5 l o s h} \approx 0.05, r_{n}=0.15 \mathrm{~m}$ (for a parabolic radial density profile) and $r_{A}=0.3 \mathrm{~m}$ anchor radius. The TEBASCO code calculates a radius of normal curvature $R_{A}=0.5 r_{A} /(Y A H)=15 \mathrm{~m}$ at the anchor midplane. Thus, the Van-Dam limit (Eq. 2.2.7) is exceeded by a factor of about 2.5. Thus, according to Eq. 1.1 in Sec. 1, the effective hot-electron beta is degraded by a factor of about 0.7 , and the effective anchor beta is $0.5(0.7)+0.05=0.4$. That may still be sufficient for interchange stability at $B_{C}=0.5$, since $F i g .2 .2-9$ shows marginal stability at $\beta_{C}=0.6$. Otherwise, the hot-electron beta couid be increased above 0.5 to compensate, provided ion adiabaticity is maintained. 

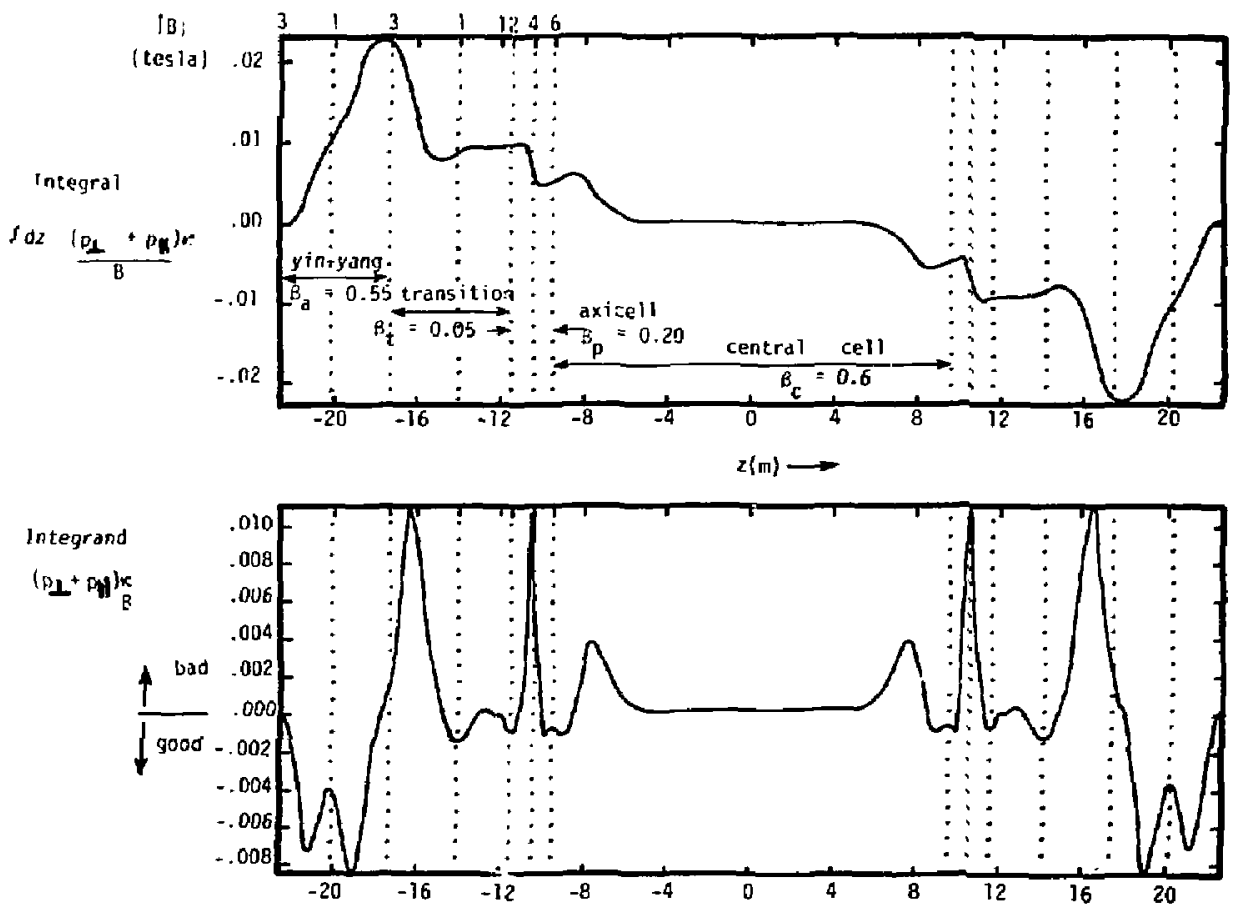

Fig. 2.2-9. MARS-mode (vacuum) flute interchange, 
Evaluation of start up of MFTF-B with thermal barriers shows that the scenario planned for TMX-U is also appropriate to MFTF-B. Therefore, for MFTF-B, we will be able to take advantage of the testing and further developing of these ideas in TMX-U. The start-up of MFTF- $B$ with therma 1 barriers in an improved axicell mode differs from start-up scenarios for MF'FF-B in an A-cell mode in one major way. Rather than build up to high censity as rapidly as possible in a tandem mode and then form the thermal barrier in an outboard A-ce11, we must form thermal bar riers at a low density $\left(<10^{12} \mathrm{~cm}^{3}\right.$ ), then continue the buildup in a thermal-barrier mode. The reason is that the hot-electron power balance requires heating electrons to mirror-conf ined energies ( $>10^{4}$ eV) at low densities; otherwise, collisional losses will clamp the electron energy below a few kiloelectron voits. ${ }^{2.7}$ With an A-cell outboard from the MHD anchor, the hot-Electron A-cell could start up at low density after the anchors and central cell were at high density. With the axice11, hot electrons for the thermal barrier are in the anchor; MHD stability then requires that the beta (throughout MFTF-B) be within an order of magnitude of the plug betas; hence, the plasma density must build up slowly throughout.

We examine start up from the viewpoint of satisfying the following set of physics constraints:

- Particle and power balance for each species in each cell.

- MHD stability.

- Microstability for both hot ions and hot electrons, and trapped-partic le modes.

For two reasons the most difficult period in which to satisfy these constraints is before thermal-barrier formation.

- First, more power is required at this time, because the plasma is confined only by magnetic mirrors, with little enhancement by electrostatic plugging.

- Second, more power is required because absolute microstability is not assured without substantial electrostatic confinement of warm ions.

We have evaluated several of these constraints at times before barrier formation and find that, even then, they define an operating window in 


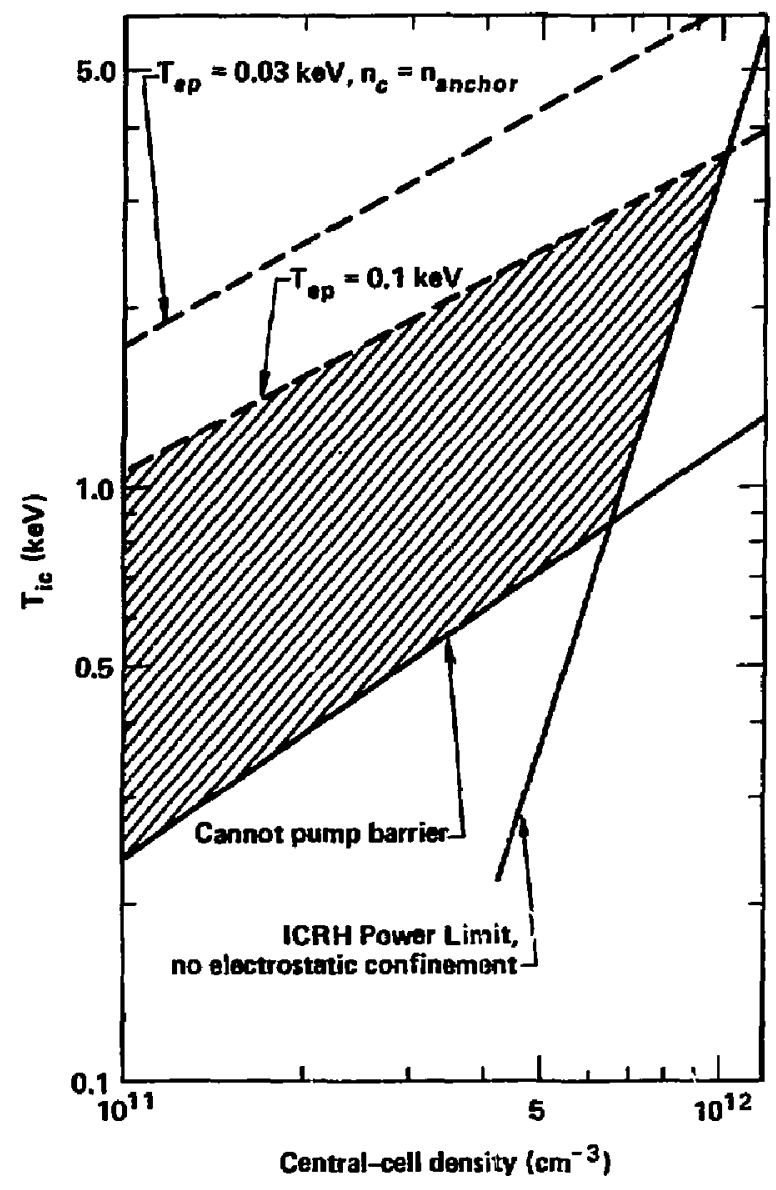

Fig. 2.2-10. Operatin:g window during start-up of MFTF-B. 


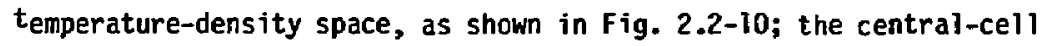

ion-particle and power balance will limit the maximum density to $\mathrm{n}=10^{12} \mathrm{~cm}^{-3}$, similar to the initial 1 imit from hot-electron power balance. The requirements for pumping the barriers provide a lower bound on the ion temperature. Hot-ion DCLC stability, because of streaming ions alone, provides a soft upper limit

to the ion temperature.

We are continuing to evaluate the other constraints; until these evaluations are complete, we will use qualitative arguments. The MHD stability was satisfactory and was less restrictive than stream stabilization of OCLC on TMX-U, and we expect similar results for MFTF-B. Stability to trapped-particle modes is less restrictive during start up than at design level, as discussed in the next paragraph. We expect the microstability of the plugs to be substantially enhanced by sloshing ions and by mirror-confined electrons, thus increasing the upper $T_{i c} 1$ imit above that resulting from microstability by streaming ions alone (see Fig. 2.2-10).

Stability to trapped-particle modes requires maintaining an adequate rátio of passing to central-cell ions. This, as well as MHD stability, will be improved during start up by keeping the central-cell density low until the arichor-plasma parameters are near design level. Then the central-cell density can be increased to its limits. The greatest susceptibility to trapped-particle modes will occur before thermal-barrier formation, when the passing-ion density will be lower because of the lack of a potential peak to reflect these ions. But, because the planned ICRH power of $400 \mathrm{~kW}$ into the plasma is four times the computed power losses during this $t i m e$, we $c$ an tolerate a substantial degradation of the confinement because of instabilities without impairing our ability to start up MFTF-B.

The plasma parameters will be kept in the operatins window by controlling the timing and the power or current of the following "knobs" as indicated in Fig. 2.2-11:

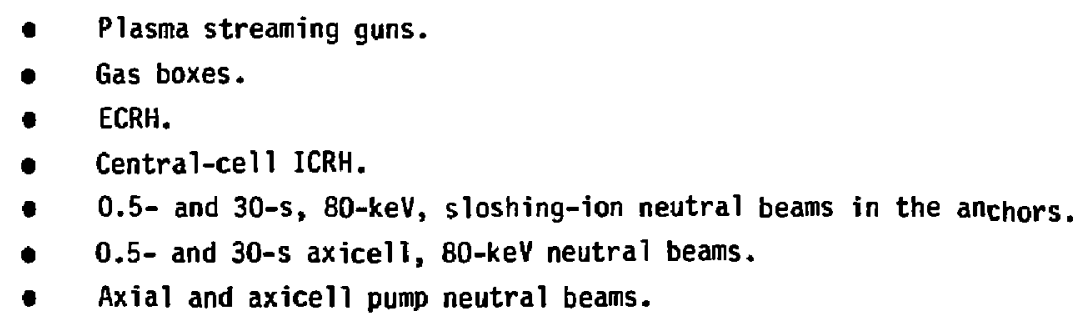



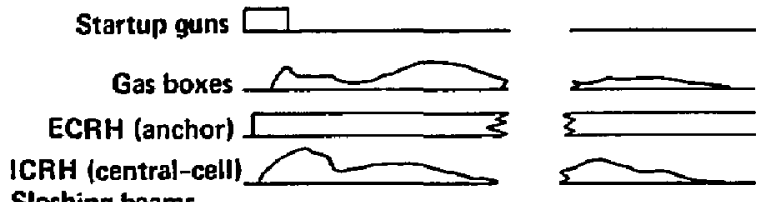

\section{Sloshing beams}

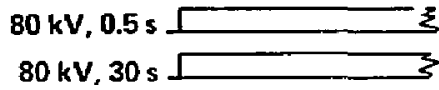

\section{ᄂ2}

$80 \mathrm{kV}, 30 \mathrm{~s}$

\section{Axicell beams}

$$
80 \mathrm{kV}, 0.5 \mathrm{~s}
$$
迄

$80 \mathrm{kV}, 30 \mathrm{~s}$

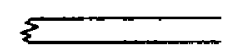

Pump beams

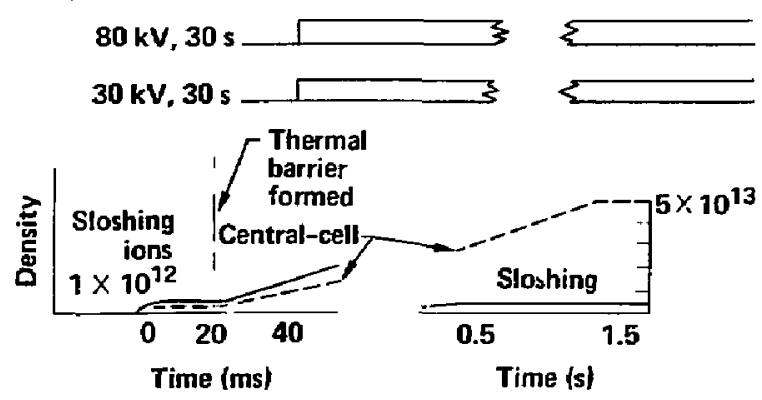

Fig. 2.2-11. Start-up of MFTF-B Axicell with thermal barriers. 
We expect the knobs to be used as follows: Plasma streaming guns will provide a seed plasma of minimum density. The ECRH will be used at maximum power to build up the hot-electron density as fast as possible. The hot electrons provide several benefits: they aid MHD stability and hot-ion microstability; they improve the operation of the thermal barriers; and they prevent the plug plasma from being destroyed by incident gas. It is the ions associated with the hot electrons that provide sufficient trapping of sloshing beams to balance charge exchange losses on gas and pump beams. In previous scenarios that lacked hot electrons, a rapid buildup to high density was required to allow the trapping rate to exceed the charge-exchange ioss rate.

Central-cell ICRH will be turned on near full power before the central-cell density exceeds $10^{12} \mathrm{~cm}^{-3}$ in order to heat the central-cell and passing ions to a low collisionality. The power level will then be programed down to keep $T_{i w C}$ be low the DCLC and MHD boundaries.

Sloshing-ion beams must be turned on at full power soon after the ICRH in order to maintain MHD stability.

The gas boxes will be turned on to fuel the plasma after the plasma streaming guns are turned off. They will be programed initially to keep the density within the window just discussed. After a thermal barrier is formed at low density, the gas will be programed to maintain the plug density sufficientiy higher than the hot-electron density to achieve hot-electron microstability.

In steady state, most of the fueling and power for the central cell will come from the axicell and pump beams. But, the gas box and central-cell ICRH are arailable during buildup so that operational flexibility is not restricted by both the pumping and the central-cell heating being tied to these beams. Pump neutral beams will be off until the other conditions for establishing a thermal barrier are either established or are within a pumping time constant (a few milliseconds) of being established. The axicell beams provide a small potential peak to reduce the fraction of the central-cell ions that become passing ions. The passing ions are further reduced by the pump beams. The pump beams must be turned on to form the thermal barrier. The axicell beams enhance the performance of the thermal barrier, so are turned on when required by the particle or power balance. 
The transition from 0.5 - to 30 -s-duration neutral beams can be staged to avoid a sudden decrease in the beam current without a simultaneous decrease in the gas associated with the $0.5-5$ beams. The gas incident on the plasma will decrease along with the beam current if one beam is turned off at a time and one gas-pumping time (approximately 50 to $100 \mathrm{~ms}$ ) is allowed before turning off another.

An approximate central-cell power balance used for start up analysis is shown in Fig. 2.2-12, both before and after thermal-.barrier formation. The maximum power requirement is $600 \mathrm{~kW}$ at design level. However, the axice 11 bealıs contribute about $200 \mathrm{~kW}$ to the central cell, leaving $400 \mathrm{~kW}$ to be supplied by the ICRH system. We plan to use the fundamental resonance, $\omega_{c i}$, for start up and initial heating because that directly heats the bulk of the ion distribution, as required to minimize collisionai filling of the thermal barrier. Evanescent fast-wave heating at $w_{c j}$ has been demonstrated on the Phaedrus tandem mirror (see discussion and references in Ref. 2.8) to couple sufficient power to meet the requirements shown in 2.2-12. After the density increases sufficiently for a fast wave to n to propagate, we expect that the fundanental resonance will no longer heat effectively. We will then use $2 \omega_{c j}$ heating. At these high densities ( $>2 \times 10^{13} \mathrm{~cm}^{-3}$ ), heating the tail of the jon distribution will not be a problem because collisions will transfer sufficient power from the tail to the bulk. An attractive way to accompiish this heating using one transmitter and an antenna is to place the antenna near the 2. $T$ fjeld of the central cell and operate the transmitter near $15 \mathrm{MHz}$. This provides heating at $\omega_{c i}$ in the near field of the antenna until the density exceeds $\sim 2 \times 10^{13} \mathrm{~cm}^{-3}$, at which point the wave will propagate to the midplane where heating will cccur at $2 \omega_{c j}$. We will continue to evaluate whether a more flexible two-frequency system might be more effective. The ICRH experiments in TMX-U, as well as in other tandem mirrors, will provide addjional data on which to base a decision.

Operation outside tlie desired window can be corrected in the following ways. Too collisional a barrier can be corrected by increasing the ICRH power or varying its $t$ iming to increase $T_{i c}$, by decreasing the gas-feed rate or start-up-gun power, or by varying their timing to decrease the density. The MHD stability can be regained by increasing the sloshing neutral-beam current, decreasing the pump-bean current, or by decreasing the current of neutral gas incident on the plasma core, all of which will increase the density of 


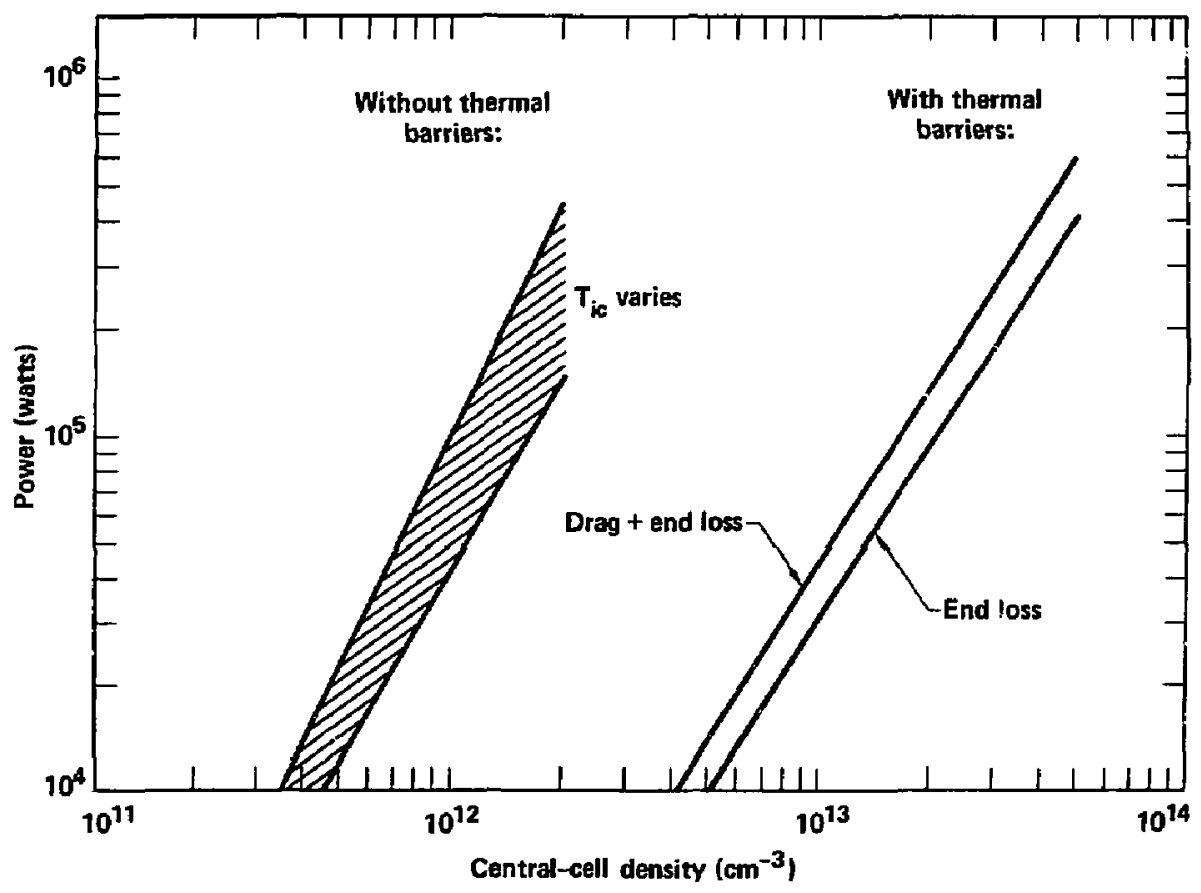

Fig. 2.2-12. Approximate central-cell power balance used for start-up. 
sloshing ions. MHD stability can also be increased by decreasing the axice!l beam current. Control of the neutral gas current reaching the plasma core may require controiling the plasina boundary density and temperature. Both MHD and DCLC stability can be regained by prograrming the ICRH power downwards.

Programing the density would have no effect if the ICRH power coupled into the plasma were exactly proportional to the density, but since the proportionality is oniy approximate, some control over $T_{i c}$ is available by varying the gas-box input. Operation at too high a density can be corrected by reducing the start up gun power or duration and by reducing the gas-box current.

\section{REFERENCES}

2.7. B. W. Stallard, Y. Matsuda, and W. M. Nevins, "Fokker-Planck Calculations of Electron Cyclotron Resonant Heating (ECRH) in Mirror Geometry", in Proc. of Second Workshop on Hot Electron Ring Physics, San Diego, CA, December 1981, LLHL Report, UCRL-86826 (1981).

2.8. A. W. Molvik and S. Falabella, Use of ICRH for Start-Up and Initial Heating of the TMX-U Central Ce11, LLNL Report, UCID-19342 (1982). 


\subsection{ALTERNATE OPERATING MODES}

\subsubsection{KELLEY TDF-LIKE MODE}

The Kelley TDF-like mode differs from the thermal-barrier MARS mode mainly by the introduction in the central cell of a neutral-beam injected, hot component that is predominantly mirror confined. The hot component dominates the neutron production because of its higher temperature and density compared to the central-cell warm component, which is potentially confined and Maxwellian. The warm component is required for microstability of the hot component. Axial profiles of $B, \phi$, and $n$ are given in Fig. 2.3-1, and the parameters are given in Table 2.3-1. The axicel) is eliminated (see MHE discussion) in the Kelley-TDF mode. Although the Kelley-TDF mode has a higher physics $Q_{c}$ than the thermal-barrier MARS mode $(0.7$ compared to 0.6$)$, the scaling associated with mirror confinement, compared to potential confinement, reverses the ordering for reactor conditions. However, as pointed out by Fowler and Logan, ${ }^{2.9}$ the physics associated with the TDF mode indicates that a tandem-mirror machine about the size of TMX could provide fusion nuclear-engineering data. The lower $t$ value $(0.16 \mathrm{~s}$ for the TDF mode compared to $1 \mathrm{~s}$ for the MARS mode) allows equilibrium to be reached with $0.5 \mathrm{~s}$ beams without the aid of $30 \mathrm{~s}$ duration beams. Here we describe for the TDF-mode the physics constraints involving microstability, MHD, thermal-barrier formation, the trapped-particle instability suggested by Berk and Rosenbluth, 2.10 and power flow.

\section{Microstabi]ity}

As with the thermal-barrier MARS mode, the microstability requirements of the anchor are satisfied by passing jons, which become potentially trapped in the anchor. The warm component within the central cell has a density level high enough to establish a monotonic distribution.

Magnetohydrodynamics (MHD)

The predicted experimental value of $B_{C}$, at a fixed $B_{C}$ value for the Kelley-TDF mode, is higher than in the thermal-barrier MARS mode because of the mirror-confined hot ions. By increasing the central-cell magnetic field from 1.0 to $1.6 \mathrm{~T}$, lowering the transition beta, and eliminating the unstable drive 


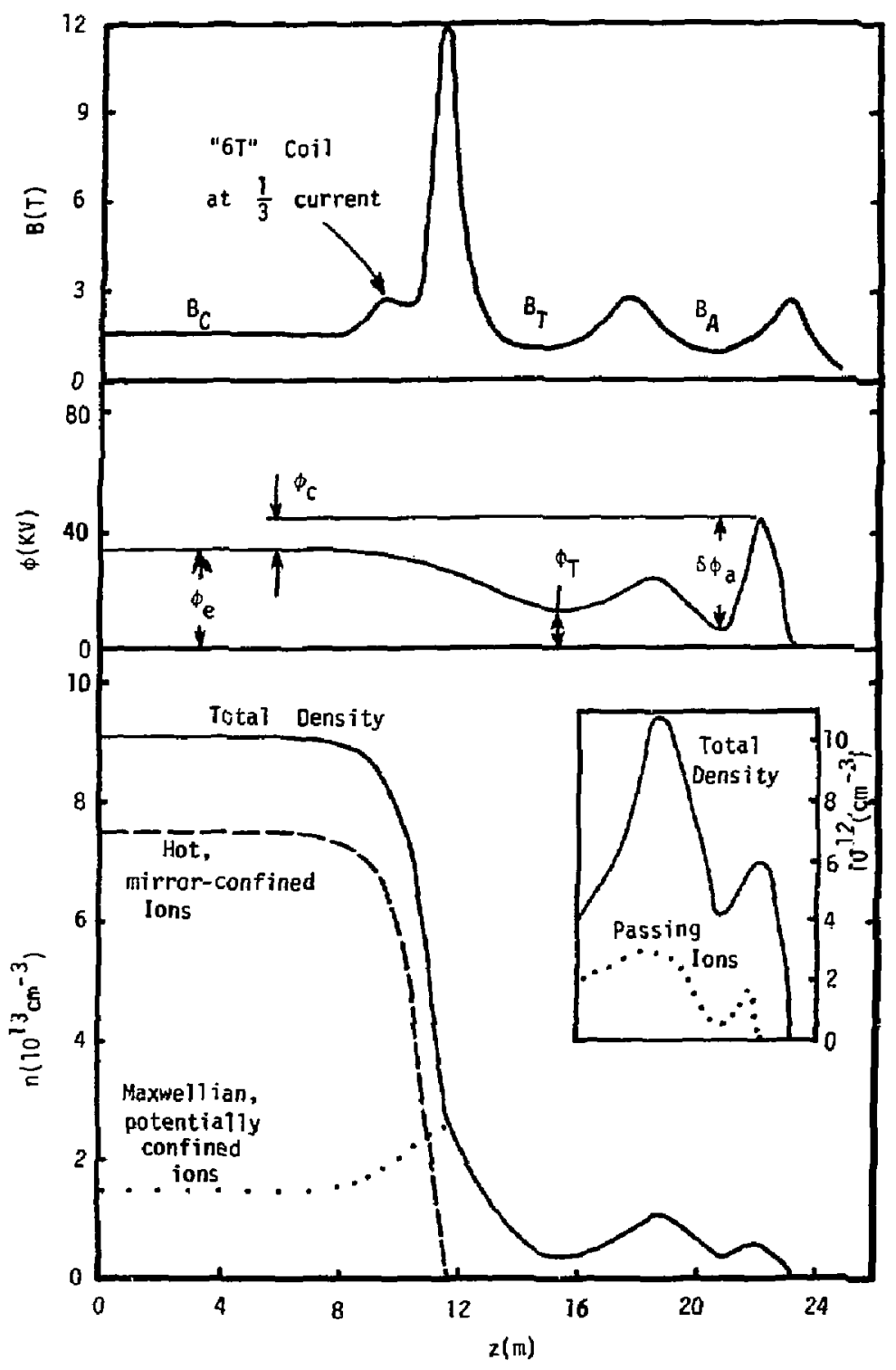

Fig. 2.3-1. Axial profiles in Kelley mode of MFTF-B Axicell. 
Table 2.3-1. Central-cell plasma parameters.

\begin{tabular}{|c|c|c|c|}
\hline Central cell (C) & $\begin{array}{c}\text { Thermal } \\
\text { barrier-MARS }\end{array}$ & Kelley-TDF & TMX-U \\
\hline${ }^{B} \mathrm{C}(T)$ & 1 & 1.6 & 1 \\
\hline$\phi_{e}(k V)$ & 50 & 34 & 50 \\
\hline$\phi_{C}(k V)$ & 30 & 11 & 30 \\
\hline$n_{e c}\left(10^{13} \mathrm{~cm}^{-3}\right)$ & 4.8 & 9.1 & 1.3 \\
\hline$n_{i w c}\left(10^{13} \mathrm{~cm}^{-3}\right)$ & 4.4 & 1.6 & 1.2 \\
\hline$n_{\text {inc }}\left(10^{13} \mathrm{~cm}^{-3}\right)$ & 0.4 & 7.5 & 0.1 \\
\hline$T_{e C}(k V)$ & 9 & 6 & 9 \\
\hline$T_{\text {iwC }}(k V)$ & 15 & $15(1), 10(11)$ & 15 \\
\hline$T_{\text {ih } C}(k V)$ & 35 & 35 & 35 \\
\hline$(n \tau)_{\mathrm{ec}}\left(10^{13} \mathrm{~cm}^{-3} \cdot \mathrm{s}\right)$ & 4 & 1.9 & 5 \\
\hline$(n \tau)_{i w C}\left(10^{13} \mathrm{~cm}^{-3} \cdot s\right)$ & 5 & 0.6 & 5 \\
\hline$(n \tau)_{\text {inc }}\left(10^{13} \mathrm{~cm}^{-3} \cdot \mathrm{s}\right)$ & 2 & 1.4 & 2 \\
\hline$P_{C}^{\text {Fusion }}(k W)$ & $640^{\mathrm{a}}$ & 4200 & 80 \\
\hline$P_{C}^{I C R H}$ trapped $(k W)$ & 210 & 0 & 60 \\
\hline$P_{C \text { trapped }}^{\text {Beam }}(k H)$ & 0 & 5600 & 0 \\
\hline${ }^{B_{C}}$ & 0.5 & 0.54 & 0.12 \\
\hline$r_{C}(\mathrm{~cm})$ & 30 & 24 & 30 \\
\hline icias trapped (Atom Amps) & 1.3 & 0 & 1.3 \\
\hline $\mathrm{Q}_{\mathrm{C}}$ & 0.6 & 0.7 & 0.6 \\
\hline QTotal & 0.4 & 0.6 & 0.06 \\
\hline $\mathrm{L}_{\mathrm{c}}^{\text {effective }}(\mathrm{cm})$ & 1650 & 2060 & 2810 \\
\hline
\end{tabular}

${ }^{a}$ An additionai $190 \mathrm{~kW}$ of fusion power are generated by the axicells. 
from the axicell, we find the theoretically predicted ${ }^{{ }_{C}} \mathrm{C}$ limit is higher than the experimental value required. The " $6-T$ " coil is energized at a current level equal to one-third the full value. Any less current results in a dip in the magnitude of $B$ before it reaches the axicell, which could lead to potential MHD problems. The " $12-T^{H}$ coil remains energized at full current to allow the plasma column to clear its smaller internal-diameter-coil case.

\section{Thermal-Barrier Formation}

The potential depth of the thermal barrier $\delta \phi_{a}$, with respect to the peak confining potential, is approximately half as large in the Kelley-TOF mode as in the thermal-barrier MARS mode ( $40 \mathrm{kV}$ compared to $70 \mathrm{kV}$ ). Therefore, less ECRH power is required for the former. For $\delta \phi_{a}$, the temperature of the potentially trapped warm electrons within the anchor is the controlling factor a long with the sloshing-ion density. The neutral-beam pumping requirements are approximately the same because the sloshing-ion density depression (3 to 1 between its turning point and midplane value) does not change between the operating modes and because the passing jon density is the same.

\section{Trapped-Particle Instability}

The physics constraints imposed by the trapped particle instability are evaluated in terms of the required passing density, npass, normalized to the central-cel1 density, ${ }^{n} \mathrm{C}$. The requirement is given by $\mathrm{Eq} \cdot 2.2 .5$, except that in the TOF mode the required value of $n_{\text {pass }} /{ }^{n} \mathrm{C}$ is increases by the ratio of the mirror-confined ion energy to the warm potentially confined ion temperature of the central ce11. On the other hand, changes in other plasma parameters (e.g., central-cell radius and magnetic field), along with a smaller value of the transition beta, lowers the value of $n_{\text {pass }} / n_{C}$ that is necessary for stability in the TDF mode to about half the value required in the MARS mode.

\section{Power F low}

The physics model developed in Sec. 2.4 can be simplified when applied to the alternate modes of MFTF-B (Kelley TDF-like and THX-U-like). All the terms involving the axiceil are eliminated and the power and particle inputs from the P2B2 pumping beams are either absent or extremely small compared to the remaining terms. A generalized power-flow diagram of the central cell, 
derived from Eq. 2.4.42 is given in Fig. 2.3-2. A hot-ion component is added to allow a beam-driven or ICRH-produced tail in the ion distribution to be present. In Table 2.3-1 the central-cell plasma parameters for the alternate modes are compared with those for the thermal-barrier MARS-mode reference.

The anchor beam and ECRH requirements for the Kelley-TDF can easily be scaled from the numbers for the thermal-barrier mode (Sec. 2.2.3), using the equations of Sec. 2.4. The hardware requirements never exceed those of the thermal-barrier mode.

The central-cell plasma $Q$ value, $Q_{c}$, is given by

$Q_{C}=\frac{E_{D-T}^{F u s i o n}}{4}\left\{\frac{\left.\left.n_{i h C}^{2}<\sigma\right\rangle^{h}+n_{i w C}^{2}<\sigma v\right\rangle^{w}+2 n_{i n C}{ }_{i w C}^{<\sigma v\rangle^{h w}}}{\frac{n_{i h C}^{2}}{(n \tau)_{i h C}^{\text {mirror }}}\left(E_{i h C}+\phi_{e}+T_{e C}\right)+\frac{n_{j w C}^{2}}{(n \tau)_{i w C}^{\text {Pastukhov }}\left(\phi_{C}+T_{i w C}+\phi_{e}+T_{e C}\right)}}\right\}$,

where a 50:50 mix of D-T is assumed for both the hot and warm species and $E_{D-T}^{\text {Fusion }}=17.6 \mathrm{MeV}$. For $T_{\text {ihc }}=35 \mathrm{keV}$ and $T_{\text {iwc }}=15 \mathrm{keV}$, we used

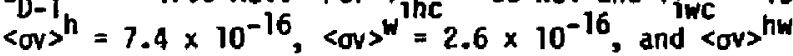
$=3.2 \times 10^{-16} \mathrm{~cm}^{3} \cdot \mathrm{s}^{-1}$. The value of $Q_{\text {Total }}$ is calculated by adding the fusion power and the power losses from the other celis.

The central-cell magnetic-field strength for the Kelley-TDF mode is at the maximum value of $1.6 \mathrm{~T}$ rather than at $1 \mathrm{~T}$, as in the thermal-barrier mode, to allow $n_{i n C} \propto B_{C} B_{C}^{2}$ to reach a maximum level. Given that the hot-ion component is generated from neutral-bean injection, the value of $E_{i h c}$ can be derived from the Logan-Mirin-Rensink model. 2.11 The MHO-limited value of ${ }^{B_{C}}$ gives in turn $n_{i h C}$, with $n_{i w C}$ determined from Fokker-Planck studies of the central-cell ion distribution.

The $\phi_{C}$ value was chosen high enough to minimize the power flow through

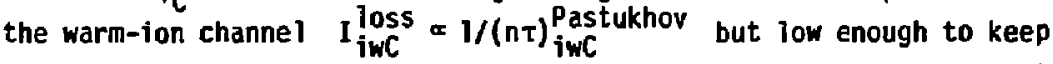
$\left(\phi_{\mathrm{e}}+\phi_{\mathrm{C}}\right)$ below the thermal-barrier mode. After determining $\left(\phi_{\mathrm{e}} / T_{\mathrm{eC}}\right)$ from equating electron- and ion-Pastukhov-particle losses, the values of $T_{i w C}$ and Tec can be determined from the power-balance equations described in Fig. 2.3-2. The trapped neutral-beam power must support the power losses associated with both the ion- and electron-particle losses. 


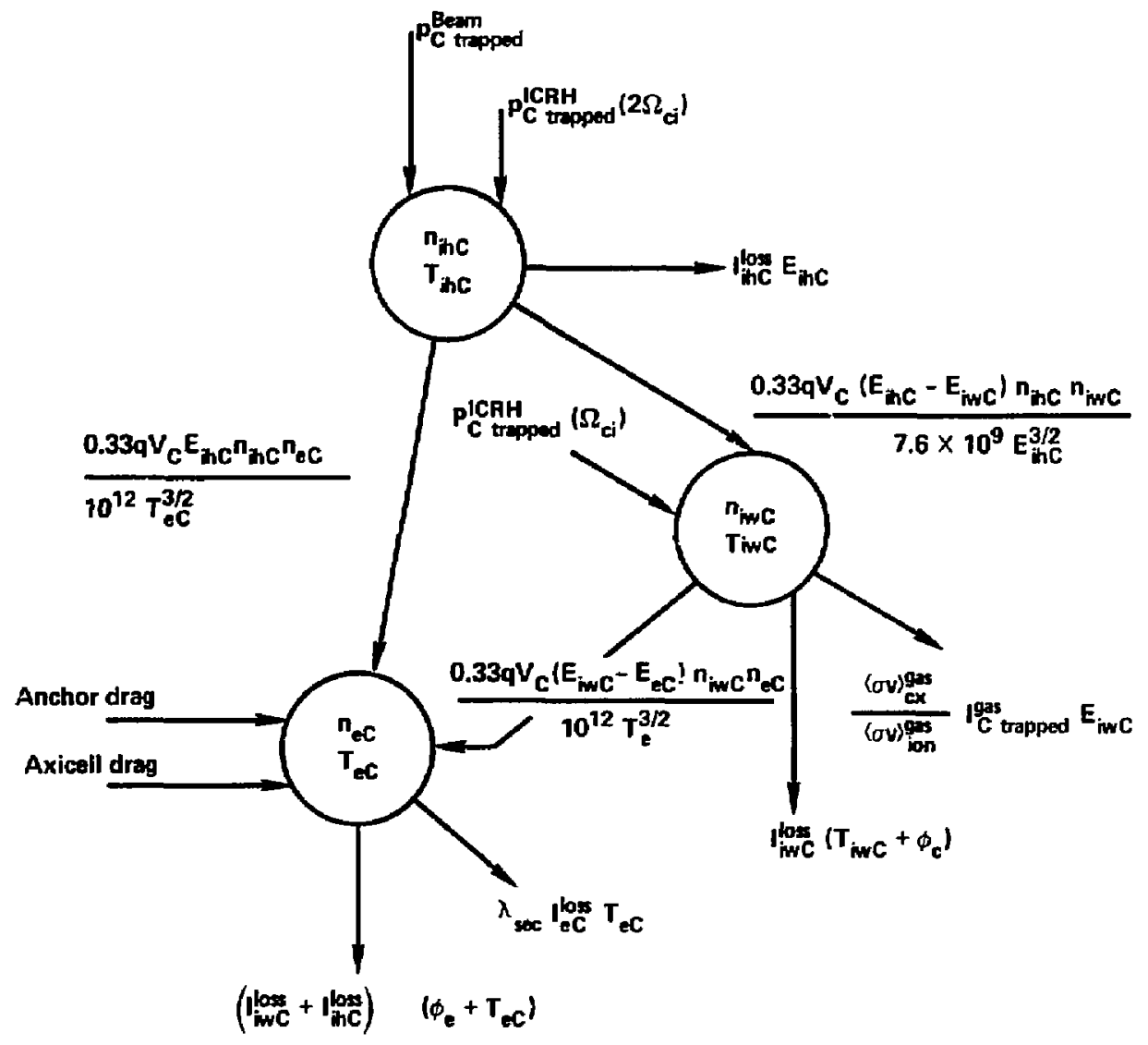

Definitions:

$$
\begin{aligned}
& \left\langle n^{2}\right\rangle=1 / 3 n^{2} \text { for } n(r)=n(0)\left[1-\left(r^{2} / r_{c}^{2}\right)\right]
\end{aligned}
$$

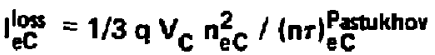

$$
\begin{aligned}
& \text { ihc }=1 / 3 q V_{c} n_{\hbar c}^{2} /(n \tau)_{h C}^{\text {mirer }} \\
& \lambda_{\text {sec }}=I^{\sec } / I_{\mathrm{ec}}^{\operatorname{loss}}
\end{aligned}
$$

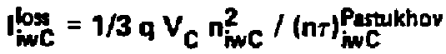

$$
\begin{aligned}
& v_{c}=\pi r_{C}^{2} L_{C}^{\text {effective }} \text { with } L_{C}^{\text {effective }} \\
& =B_{\text {mid }} \int \mathrm{d} l / \mathrm{B}
\end{aligned}
$$

Fig. 2.3-2. Central-cell power flow for alternate modes. 
The pumping requirements in the transition are no greater than the therma1-iarrier numbers because the warm-ion losses are less in the Kelley-TDF mode. The ECRH heating is also less since $\left(\phi_{e}+\phi_{C}\right)$ is approximately half the thermal-barrier value. No gas-current feed is required because of the large amount of trapped neutral-beam current that eventually joins the warm-ion distribution.

\subsubsection{TMX-U-LIKE MODE}

The TMX-U-like mode is very similar to the thermal-barrier MARS mode in terms of $B, \Phi_{e}, \Phi_{C}$, and $(n \tau){ }_{\text {iwC }}$ but operates at lower $n_{\text {jwC }}\left(1.2 \times 10^{13}\right.$ compared to $4.4 \times 10^{13}$ ). The axial profiles of $B, \phi$, and $n$ are given in Fig. 2.3-3, and the parameters are given in Table 2.3-1 of Sec. 2.3.1. The TMX- $U$ mode differs from the previously discussed MARS and TOF modes by the absence of neutral-beam pumping within the transition region between the central cell and the anchor. The only neutral beams required are those associated with the anchor. Because of the long particle lifetimes (approximately $4 \mathrm{~s}$ ), the 0.5 -s beams are not able to support this experiment a lone. Long pulse (30-5) beams and ECRH and ICRH are required but at no larger power levels than for the thermal-barrier mode.

\section{Microstability}

The plasma parameters within the anchor that are associated with the sloshing ions and ECRH electrons are identical in the TMX-U and MARS modes. The passingmion density, however, at the inside 3-T mirror of the anchor is four times greater for the TMX-U mode $\left(1.2 \times 10^{13} \mathrm{~cm}^{-3}\right.$ compared to $0.3 \times 10^{13} \mathrm{~cm}^{-3}$ ). All microstability requirements of the anchor are, therefore, easily satisfied. The central cell has only a small percentage of hot ions (less than 10\%). This amount does not perturb the inherent microstability of the Maxwellian distribution, which is potentially confined.

\section{Magnetohydrodynamics (MHD)}

The theoretically predicted 1 imit of $\beta_{c}$ is lower in the TMX-U mode ( $12 x$ compared to $50 x$ in the MARS mode) because of the increase in the destabilizing drive associated with having the transition density, $n_{T}$, equal to the central-cell density, ${ }^{n_{C}}$. 


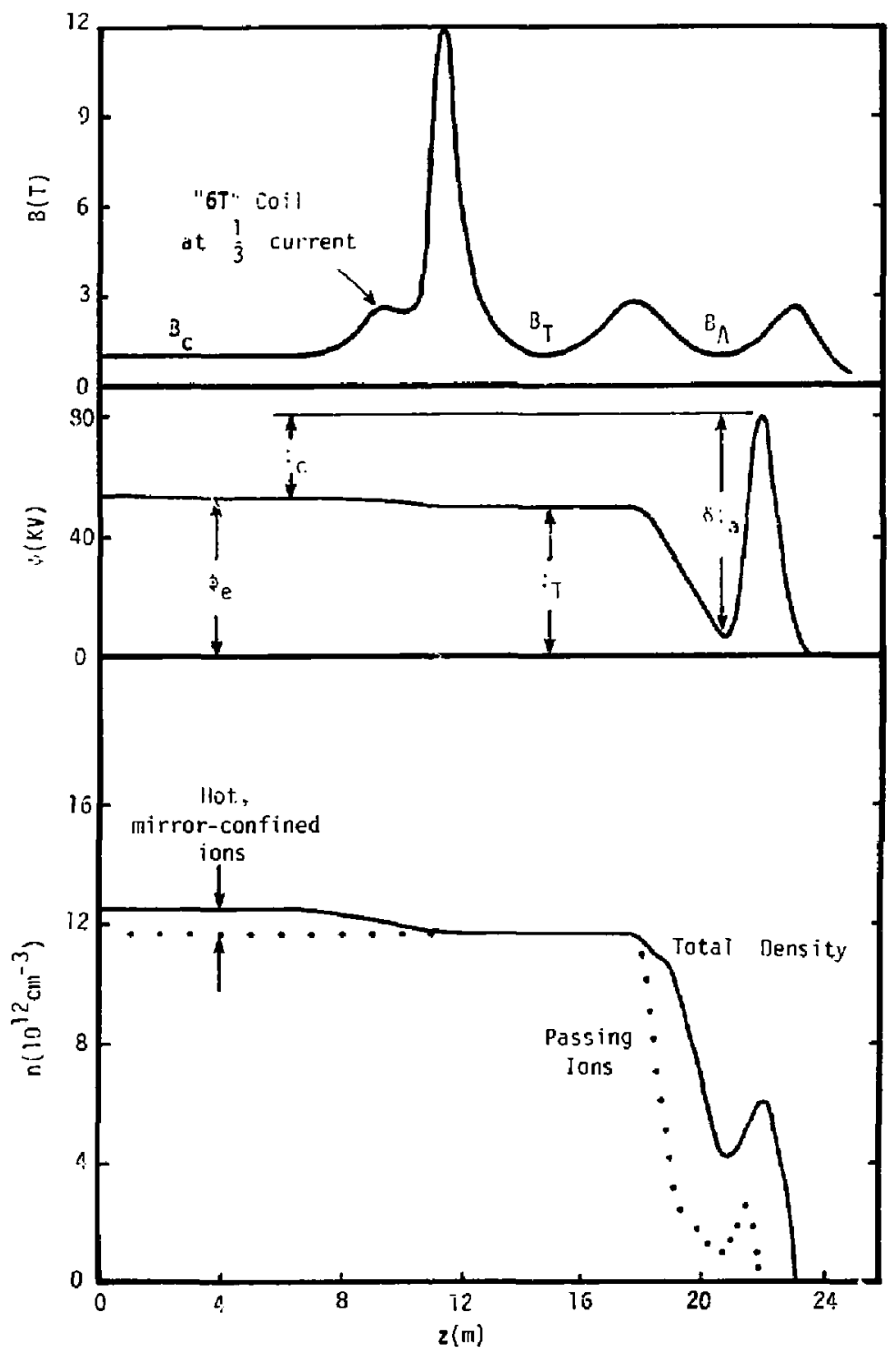

Fig. 2.3-3. Axial profiles in TMX-U mode of MFTF-B Axicell. 


\section{Thermal-Barrier Formation}

Because the anchor parameters are the same for the TMX-U and MARS modes, the barrier formation will be the same in terms of ECRH power. The increased passing oensity mentioned in the microstability discussion might require additional neutral-beam pumping.

\section{Trapped-Particle Instability}

Because the "worst picture" of this instability allows the amplitude of the fluctuations to locate where the growth rate is maximized and because the anchor parameters are the same in the TMX-U mode, the minimum $n_{\text {gass }} / n_{C}$ required for stability (and hence maximum $n_{C}$ ) is the same in the $T M X-U$ and MARS modes. However, since barrier pumping only occurs in the yin yang, the 12-T coil does not affect $n_{C}$, which is limited to a value four times lower in the TMX-U mode compared to the MARS mode. Thus, trapped-particle stability is satisfied by a margin of four.

\section{Power Flow}

The long particle lifetime $(\sim 4 \mathrm{~s})$ requires the duration of power input into the central cell to exceed the 0.5-s-duration beams. The ICRH power available (see Sec. 2.2.3) for the thermal-barrier MARS mode more than satisfies the needs of the TMX-U mode. The amount of trapper ICRH power, $P_{C}^{I C R H}$ trapped, necessary to support $T_{i w C}$ of $15 \mathrm{keV}$ is estimate by using the Logan-Mirin-Rensink model (Ref. 2.11) to calculate $E_{i n c}$ ani by using the power flow in the warm-ion channel (see Fig. 2.3-1). Central-cel? particle losses from charge-exchange with the high-energy pump beam and from passing particles trapped within the anchor have been ignored. Enough ICRH power is available so that additional power losses can be met if these che ge-exchange losses become important or if the $E_{\text {inc }}$ estimate is inappropriate for ICRH heated plasmas. The value of $P_{C}^{I C R H}$ trapped does not scale with $n_{i W C}^{2}$ because a large fraction of the power input into the central call in the thermal-barrier case came from the axicell beam and the transition Juning beam (see Eq. 2.4.42 in Sec. 2.4).

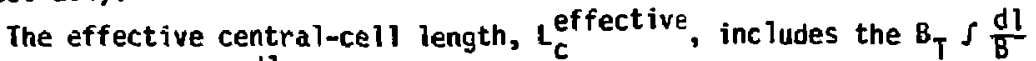
contribution to $B_{C} \& \frac{d l}{B}$, because the density is uniform from the central cell through the transition (see Fig. 2.3-3), as is the case for the present TMX-U experiment. 


\subsubsection{TARA-LIKE MODE}

In the event some as-yet undiscovered effect other than charge separation can stabilize the trapped particle mode, it would be prudent to design the axicell in MFTF-B in such a way as to allow conversion to the TARA-like configuration (see Ref. 2.1), to hive the thermal barrier generated in a larger mirror ratio axice11, and to require a minimum of conversion time and cost. At present, we require the 6-T and $1 \bar{c}-T$ axicell coils for the MARS-mode to be usable in a TARA-mode; we switch their order along the z-axis and spreading them apart from 2 to $4 \mathrm{~m}$ to allow ECRH at I T and sloshing-ion injertion into larger nimrar ratio. Figure 2.3-4 shows an early MFTF-B design for a TARA-configuration magnet set, along with the appropriate axial profiles of field, density, and potential. Corresponding plasma parameters for this early TARA-mode set are given in Table 2.3-2.

The only differences between this early TARA-mode design and one suitable with the present magnet set converted to the TARA-configuration would be that the yin yang would have a mirror ratio of $3: 1$ instead of $2: 1$, and a lonser length of $5.2 \mathrm{~m}$ instead of $3.4 \mathrm{~m}$, as well as a slightly shorter central cell (with 14 soienoid coils as planned). Since the midplane yin-yang field $\left(B_{A}=1 T\right)$ and betia value $\left(\beta_{A}=0.5\right)$ would be the same, anchor parameters might be similar to those given in Table 2.3-2.

In the conversion to the TARA-mode, the perpendicular axicell beam of the MARS-mode is expected to remain approximately in place and serve as a sloshing-ion bean when the inner axicell mirrors are moved further toward the central cell to get the desired mirror ratio. The axicell beams thus would hit perpendicularly at a mirror ratio of 1.5 to 2 to make sloshing jons. The 30-kV pump beams (P2B2), however, would have to be translated about $4 \mathrm{~m}$ towards the central cell (still at about $30^{\circ}$ angle) to pump the axicell. Moving ECRH from the yin yangs to the axicells would then mostly complete the conversion to the TARA-mode. Present best estimates are that it will take a few months and about two million dollars to make the conversion.

Because the estimated achievable plasma parameters and performance levels (Q) of this early design for the TARA-configuration (Table 2.3-2) are roughly comparable to the reference-case MARS-mode (Table 2.2-6), what would be the advantajes of the TARA-mode compared to the MARS-mode? One advantage would be that radial transport of central-cell ions that are due to the 

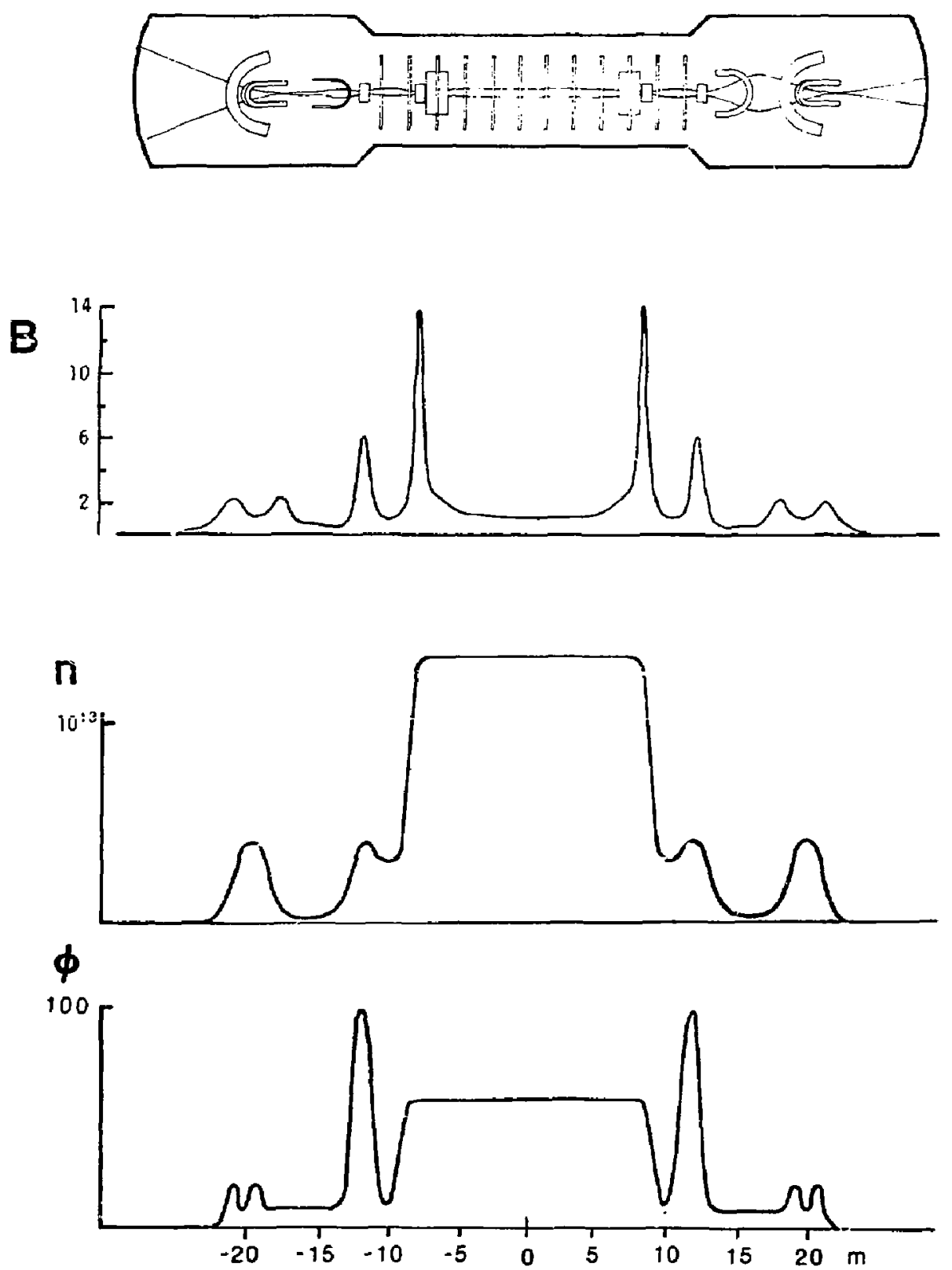

Fig. 2.3-4. Axial profiles in the TARA-like configuration of MFTF-B. 
TABLE 2.3-2. Parameters for MFTF-B Axicel1 in the TARf-?ike mode (for an earlier design), $Q=0.37, Q_{c}=1, P_{\text {fusion }}=330 \mathrm{kH}$.

Central cell:

$\begin{array}{lll}B_{c}=1 T & n_{i h}=1.95 \times 10^{12} & T_{i c}=20 \mathrm{keV} \\ L_{c}=12.5 \mathrm{~m} & B_{c}=.356 & T_{\mathrm{ec}}=12 \mathrm{keV} \\ r_{c}=30 & \phi_{c}=33.7 & \left(\mathrm{n \tau}_{\mathrm{c}}=5 \times 10^{13}\right. \\ n_{i c}=2.78 \times 10^{13} & \phi_{e}=44.1 & \end{array}$

Axicell barrier:

$\begin{array}{lll}B_{b}=1 T & T_{e w}=60 \mathrm{keV} & n_{i a}=6.89 \times 10^{12} \mathrm{~cm}^{-3} \\ B_{x i}=6 T & B_{b}=0.11 & \phi_{b}=38.1 \mathrm{kV} \\ B_{x 0}=6 T & F_{e c}=0.13 & \delta \phi_{a}=71.8 \mathrm{kV} \\ L_{x}=4 \mathrm{~m} & n_{b}=3.59 \times 10^{12} \mathrm{~cm}^{-3} & I_{\text {trap }}=0.670 \mathrm{~A} \\ E_{e h}=88 \mathrm{keV} & & \end{array}$

Beam requirements:

$$
\begin{aligned}
& I_{\text {loss }}=2.93 \mathrm{~A} \\
& I_{C}(\text { ion })=0.776 \mathrm{~A}
\end{aligned} \quad I_{\text {slosh }}(\text { abs })=0.470 \mathrm{~A} \quad I_{\text {anchor }}(a b s)=0.4 \mathrm{~A}
$$

Microwave power:

$$
\begin{aligned}
& \mathrm{P}_{\mathrm{a}}=81.9 \mathrm{~kW} \\
& P_{\text {ANCHOR }}=39.4 \mathrm{~kW} \\
& P_{\text {CC }}(\text { ICRH })=47.3 \mathrm{kH} \\
& P_{b}=86.1 \mathrm{~kW}
\end{aligned}
$$


quadrupole anchor would be negligible in the TARA-mode but still be significant in the MARS-mode. On the other hand, some radial transport is beneficial for impurity remova1. Perhaps the main advantage of the TARA-mode would be to test the idea that MHD anchors might still work even though isolated from the confined axicell plasma, thus permitting evolution of muci. simpler and lower field anchors. This, after all, was one of the main attractions of the original TARA concept.

\section{REFERENCES}

2.9. T.K. Fowler and :.G. Logan, Tandem Mirror Technology Demonstration Facility, LLNL Report, UCID-19193 (1981).

2.10. M. N. Rosenbluth, H. L. Berk, H. Y. Hong, D. E. Baldwin, and T. Antonsen, "Fast-Growing Trapped Particle Modes in Tandem Mirrors;" also L. D. Pearlstein, D. E. Baldwin, R. H. Cohen, T. K. Fowler, and B. 6. Logan, "Stabilization of Tandem Mirror Trapped Particle Modes by Incomplete Cancellation of Particle Drifts." Both papers presented at the 1982 Sherwood Meet ing, Annual Controlled Fus ion Theory Conference, Santa Fe, $\mathrm{NM}$, Apri1 25-28, 1982.

2.11. B.G. Logan, A.A. Mirin, and M.E. Rens tucl. Fusion 20, 1613 (1980). 


\subsection{PHYSICS MODELS FOR CALCULATIONS OF PARAMETERS}

The plasma parameters for the axicell version of MFTF-B shown in Table 2.3-2 were calculated using the following physics models, which are similar to those used in determining the operating scenario for the A-cell version of MFTF-B. ${ }^{2.12}$ Where further model developments and Fokker-Planck calculations occurred, we incorporated the improvements in the present calculation. The models were developed for the MARS-like mode but $c$ an be used with suitable modifications for the other modes.

\subsubsection{CENTRAL-CELL PARAMETERS}

As in the earlier calculation, ${ }^{2.12}$ we chose a design point based on a set of central-cell parameters that are near the limit of MHD stability for our magnet design. ${ }^{2.13}$ In this case, we choose a central cell ${ }^{B} \mathrm{C}$ of 0.5 with an axicell $\beta_{x}$ of 0.20 . To maximize the fusion power achievable from a 50:50 D-T mix, we chose the thermal-ion temperature in the central cell to be $T_{\text {iwC }}=15 \mathrm{keV}$. The electron temperature is estimated to be about $60 \%$ of this value (as in Ref. 2.12), and hence $T_{e c}=9 \mathrm{keV}$. The desired ion Pastukhov confinement parameter is chosen as $(n \tau)_{i w C}=5 \times 10^{13}$, and this Jetermines the required ion-confining potential $\phi_{C}$. This value of $\phi_{C}$ is near the maximum that can be generated with $80-k V$ beams and ECRH.

The electron-confining potential $\phi_{\mathrm{e}}$ is initially chosen to give an electron Pastukhov (nT) equal to the ion value. This tends to give an cverestimate of $\phi_{e}$ because the presence of secondary electrons flowing in from the end walls would produce an electron-confinement time that is less than the ion Pastukhov-confinement time. However, this estimate for $\phi_{e}$ is a satisfactory starting value that can be easily refined in one iteration, as will be shown later. The nomenclature used in this report is similar to that used in Rer. 2.12 and follaws the axicell system shown in fig. 2.1-2.

\subsubsection{AXICELL PARAMETERS}

The plasma density and potential profile in the axicell as viell as the neutral beam required are calculated in a manner similar to that of Ref. 2.12. The potential rise from the cintral cell to the axicell midplane, $\Delta \Phi_{D C}$, is calculated from 


$$
\Delta \phi_{p c}=T_{e c} \ln \left(\frac{n_{e x}}{n_{e c}}\right)
$$

where the electron density at the axicell midplane is determined from,

$$
n_{\mathrm{eX}}=n_{\mathrm{iwX}}+n_{\mathrm{ihX}} \text {. }
$$

The warm-ion density is assumed to be nearly Maxwellian. Hence,

$$
n_{i w X}=n_{i w C} e^{-\Delta \phi_{p c} / T} i w C
$$

The hot ions are determined by assuming the perpendicular $B$ in the axice 11 is given by the MHD-stability limit. Then

$$
n_{i h X}=\frac{2.5 \times 10^{15} B_{\perp} B_{X}^{2}-n_{i w X}\left(T_{i w C}+T_{e C}\right)}{\left(0.9 E_{i h X}+T_{e c}\right)} .
$$

The solution of Eqs. 2.4.1 to 2.4.4 gives $\Delta \phi_{p c}$ and the plasma density in the axicell.

The potential drop from the axicell midplane to the outboard mirror is calculated from

$$
\Delta \Phi_{\mathrm{pb}}=T_{\mathrm{eC}} \ln \left[\frac{n_{\mathrm{eX}}}{n_{\mathrm{ex}}(\mathrm{m})}\right] \text {. }
$$

where $n_{e X}(m)$ is the electron density at the outboard axicell mirror. This density is taken as a factor $g_{b}(m)$ times the cold-passing-ion density at the mirror given by $n_{i x}^{\text {pass }}(m)$ where,

$$
\begin{aligned}
n_{i X}^{p a s s}(m)= & n_{i c} e^{-\Delta \phi_{p c} / T_{i w}}\left\{e^{\Delta \phi_{p b} / T_{i w}} \text { erfc }\left[\left(\frac{\Delta \phi_{p b}}{T_{i w}}\right)^{1 / 2}\right\}\right. \\
& \left.+2\left(\frac{R_{0}-1}{\pi}\right)^{1 / 2} D\left[\left(\frac{\Delta \phi_{p b}}{T_{i w}\left(R_{0}-T\right)}\right)^{1 / 2}\right]\right\} .
\end{aligned}
$$

where

$$
R_{0}=B_{m \times 0} / B_{x} \sqrt{7-B_{x}} \text {, }
$$


and Dawson's integral is

$$
D(x)=e^{-x^{2}} \int_{0}^{x} e^{u^{2}} d u
$$

The plasma lifetime is calculated using the Logan-Rensink model as in Ref. 2.12. Assuming that the hot-ion lifetime is determined by scattering losses and electron drag and also that charge-exchange losses are negligible, the hot-ion-conf inement paraneter is given by

$$
(n \tau)_{p}=\left\{(n \tau)_{S}^{-1}+\left[(n \tau)_{D} \text { in }\left(E_{i n j} / \vec{E}_{L}\right)\right]^{-1}\right\}^{-1},
$$

where

$$
\begin{aligned}
& (n \tau)_{s}=\frac{3.9 \times 10^{12}\left(E_{i n j}\right)^{3 / 2}}{\ln \Lambda_{i j}} \log _{10}\left(R_{e f f}\right), \\
& R_{e f f}=\frac{B_{m \times i} / B_{X}}{\left(1+\frac{\Delta \phi_{p c}}{E_{i n j}}\right) \sqrt{1-\beta_{X}}}, \\
& (n \tau)_{D}=\frac{2 \times 10^{13}}{\ln \Lambda_{e i}} T^{3 / 2},
\end{aligned}
$$

and $E_{\text {inj }}$ is the beam-injection energy. The average energy of the ions escaping from the yin yang is $\bar{E}_{1}$ and is obtained from,

$$
\begin{aligned}
& \frac{E_{L}}{E_{i n j}}=\frac{1}{1+\tau_{s} / \tau_{D}}+\frac{E_{p}\left(\tau_{s} / \tau_{D}\right) / E_{i n j}}{1+\tau_{s} / \tau_{D}}, \\
& \frac{\tau_{s}}{\tau_{D}} \simeq \frac{0.11}{\sqrt{A}}\left(\frac{E_{i n j}}{T_{e c}}\right)^{3 / 2} \frac{\log \left(R_{e f f}\right)}{\ln \left(E_{i n j} / E_{L}\right)} \frac{\ln \Lambda_{e i}}{\ln \Lambda_{i i}},
\end{aligned}
$$

where $:$ is the ion mass in amu and

$$
E_{p}=\frac{\Delta \phi_{p c}}{\left(\frac{B_{m x i}}{B_{x}}\right)-1} \text {. }
$$


The calculation of $\Delta \phi_{p c}$ and $\Delta \phi_{p b}$ required a value of $E_{i h X}$, the hot-ion energy. This energy can be calculated by balancing the drag losses of the ions on the electrons with the net energy injected by the axicell beams. Neglecting charge exchange, the hot-ion energy is given by

$$
E_{i h X}=\left(E_{i n j}-E_{L}\right) \frac{(n \tau)_{d r a g}}{(n \tau)_{p}}+E_{e C} .
$$

Since the hot-plasma-conf inement parameter $(n \tau)_{p}$ and hence $E_{i h X}$ depends on $\Delta \phi_{p c}$, and because $\Delta \phi_{p c}$ depends on the value of $E_{i n X}$, it is cicar that an iterative procedure is required to solve for the potential profile and plasma-conf inement parameter $(n \tau)_{p}$.

The axicell beam current required to maintain the axicell is given by balancing the scattering losses,

$$
I_{x \text { ion }}=q \frac{n_{\text {ihx } x} n_{e x} v_{x}}{(n \tau)_{p}} .
$$

\subsubsection{BARRIER PARAMETERS}

With our choice of $\beta_{b}$, the beta in the midplane of the anchor, and $\phi_{e}$, we can solve for the barrier potential $\phi_{b}$ as a function of the fraction $F_{e c}$ of the cold electrons at the barrier midplane by applying a quasi-rieutrality relation at the barrier midplane. As in Ref. 2.12, the warm-passing-ion density is mapped from the central-cell value, accounting for the variation in magnetic field and the change in potential. The cold-electron-clensity distribution in the barrier mioplane region is assumed to follow a cut-off distribution,

$$
n_{e c A}\left(\phi_{b}\right)=g_{b}(m) n_{i w X}(\text { mirror })\left(\frac{e^{-\frac{\phi_{b}-\Delta \phi_{p b}}{T_{e c}}-e^{-\phi / T_{e c}}}}{1-e^{-\phi / T_{e c}}}\right),
$$

where

$$
\phi=\phi_{e}+\Delta \phi_{p c}-\Delta \phi_{p b}
$$

The density thus equals the electron density at the 12-T wirror when $\phi_{\mathrm{b}}=\Delta \phi_{\mathrm{pb}}$, and is zero when $\phi_{\mathrm{b}}=\phi_{\mathrm{e}}+\Delta \phi_{\mathrm{pc}}$. 
The quasi-neutrality relation, which is solved for the barrier potential, then is

$$
n_{e c A}\left(\phi_{b}\right)=n_{i}^{\text {pass }}(b) G_{b} F_{e c} \text {, }
$$

where the passing-ion density $n_{i}^{\text {pass }}(b)$ is scaled from the central-cell density by

$$
\begin{aligned}
& \frac{n_{i}^{p a s s}(b)}{n_{i \omega C}}=e^{-\Delta \phi_{p c} / T_{i w C}}\left\{e^{\phi_{b} / T_{i W C}} \operatorname{erfc}\left[\left(\frac{\phi_{b}}{T_{i \omega C}}\right)^{1 / 2}\right]\right. \\
& -\sqrt{1-R_{0}}\left\{e^{\frac{\phi_{b}}{T_{i W C}\left(1-R_{0}\right)}} \operatorname{erfc}\left[\left(\frac{\phi_{b}}{T_{i W C}\left(1-R_{0}\right)}\right)^{1 / 2}\right\}\right. \\
& \left.-e^{\frac{\phi_{b}+E^{\prime}}{T_{i w C}}} \operatorname{erfc}\left[\left(\frac{\varepsilon^{*}+E^{\prime}}{T_{i w C}}\right)^{1 / 2}\right]\right\} \\
& \left.-\sqrt{1-R_{m}} e^{\frac{\phi_{b}-\varepsilon^{\star}+\phi^{\star}}{T_{i w C}}} \operatorname{erfc}\left[\left(\frac{\phi^{\star}}{\mathrm{T}_{i w C}}\right)^{1 / 2}\right]\right\}
\end{aligned}
$$

where

$$
\begin{aligned}
& R_{0}=\frac{B_{b} \sqrt{T-B_{b}}}{B_{x} \sqrt{1-B_{x}}}, \\
& R_{m}=\frac{B_{b} \sqrt{1-B_{b}}}{B_{m} x_{0}}, \\
& \varepsilon^{*}=\frac{R_{0} \phi_{b}-R_{m}\left(\phi_{b}-j_{p b}\right)}{R_{0}-R_{m}}, \\
& \phi^{*}=\varepsilon^{*}+\frac{R_{m}\left(\phi_{b}-\Delta \phi_{p b}\right)}{T-R_{m}},
\end{aligned}
$$

83 


$$
\begin{aligned}
& \phi^{\prime}=\frac{R_{0} \phi_{b}}{R_{0}-1}-\varepsilon^{\star}, \\
& \varepsilon^{\prime}=\frac{R_{0} \phi_{b}}{1-R_{0}},
\end{aligned}
$$

and erfc $(x)$ is the complementary error function of $x$. Here, $B_{b}$ is the magnetic field at the barrier (anchor) midplane, $B_{X}$ is the field at the axicell midplane, and $B_{m X_{0}}$ is the outer (12-T) mirror of the axicell.

Equation 2.4.13 is solved for the barrier potential $\phi_{b}$. Then the passing density at the barrier midplane is given by $E q \cdot 2,4.14$ while the trapped-and total-ion densities at the barrier midplane are obtained from

$$
\begin{aligned}
& n_{i}^{\operatorname{trap}}(b)=\left(g_{b}-1\right) n_{i}^{\text {pass }}(b), \\
& n_{i A}(b)=G_{b} n_{i}^{\text {pass }}(b),
\end{aligned}
$$

where $g_{b}$ is the ratio of total warm-ion to passing-ion density, and $G_{b}$ is the ratio of total ion density to passing-ion density.

The hot-electron eriergy is determined by assuming that the ions and hot electrons at the barrier midplane supply a known value for $B$. Hence, for energy in kev,

$$
\begin{aligned}
E_{e h A}= & \left(2.5 \times 10^{15} B_{b} B_{b}^{2}-E_{1}(a) \frac{B_{b}}{B_{A}} \sqrt{1-B_{b}} n_{i h A}\right. \\
& \left.-T_{i w} \frac{B_{b} \sqrt{1-B_{b}}}{B_{m A i}} g_{b}(b) n_{i w A}\right) /\left[n_{i A}(b)\left(1-F_{e c}\right)\right],
\end{aligned}
$$

where $B$ is in tesla and the density is per cubic centimeter.

\subsubsection{WARM-E:ECTRON AND CONFINING-POTENTIAL PARAMETERS}

The peak in the potential profile for the anchor occurs at the position shown as a in Fig. 2,1-2. The petential and density peak are produced by $90^{\circ}$ injection of the sloshing ions at the 1.2 tesla point in the vacuum field. Fokker-Planck calculations 2.14 indicate that the potential and 
density peak occur inboard of the injection point. The potential peak is enhanced by auxiliary electron heating by microwave power applied near the 1.2-T position.

As in Ref. 2.12, the warm-electron density at a is obtained from a quasi-neutrality condition,

$$
n_{\text {ewA }}(a)=n_{i A}(a)-n_{e h A}(a)-n_{e c A}(a) .
$$

The hot-ion density at a is taken to be a factor $R_{s} t$ imes the density at the barrier midplane. As shown in Figs. 2.4.1 and 2.4.2, Fokker-Planck

calculations indicate that $z$ value of $R_{s}=2.8$ can readily be achieved. The hot- and cold-electron densities at $\underline{a}$ are mapped from the known values at $\underline{b}$, using

$$
n_{e h A}(a)=n_{e h A}(b)\left(\frac{B_{m A o}-B_{a}}{B_{m A 0}-B_{b}}\right)\left(\frac{E_{e h_{11}}}{\delta_{a}+E_{e h}}\right)^{1 / 2}
$$

and

$$
n_{e c A}(a)=n_{e c A}(b) \frac{B_{a}}{B_{b}}\left(\frac{T_{e c A}}{T_{e c A}+n_{a}}\right)^{1 / 2} .
$$

$E_{\text {ehlt }}$ is the parallel component of the hot electron energy at $\underline{b}$ and is assumed to be about $20 \%$ of the total hot-electron energy.

The confining potential for the warm electrons, $\delta \varphi_{a}$, is defined as

$$
\delta \phi_{a}=\phi_{c}+\phi_{b}-\Delta \phi_{p c} .
$$

If we use the model of Ref. 2.1, the warm-electron temperature can then be determined from

$$
\frac{\delta \phi_{a}}{\tau_{e W A}}=\ln \left(N_{C W} \frac{1+J_{A B}}{1+N_{C W} J_{A B}}\right) .
$$

as a function of the cold-electron ionization source $\mathrm{J}_{\mathrm{e}^{*}}$ In Eq. 2.4.22,

$$
J_{A B}=\frac{\sqrt{\pi}}{4}\left(1+\frac{B_{b}}{B_{a}}\right) \frac{\tau_{e e} J_{e}}{n_{e w}(a)}
$$




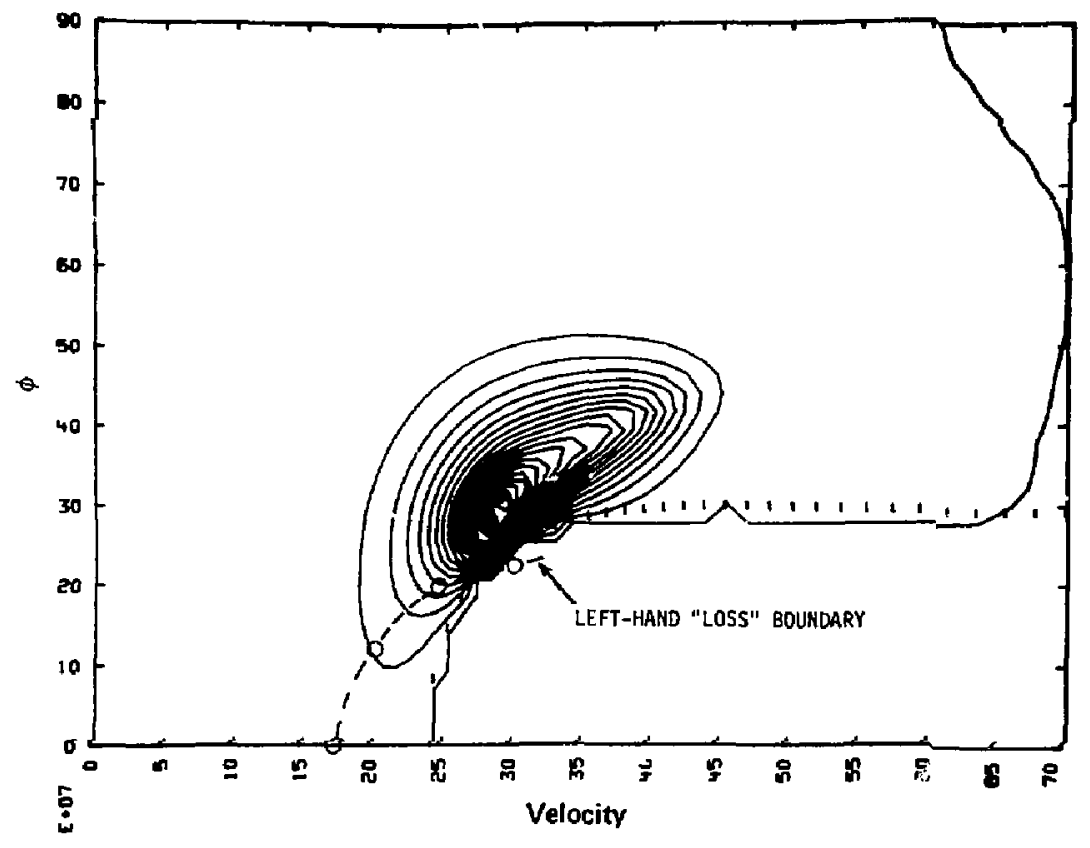

Fig. 2.4-1. Loss boundary and distribution-function contours for sloshing-ion injection in yin-yang anchor. 

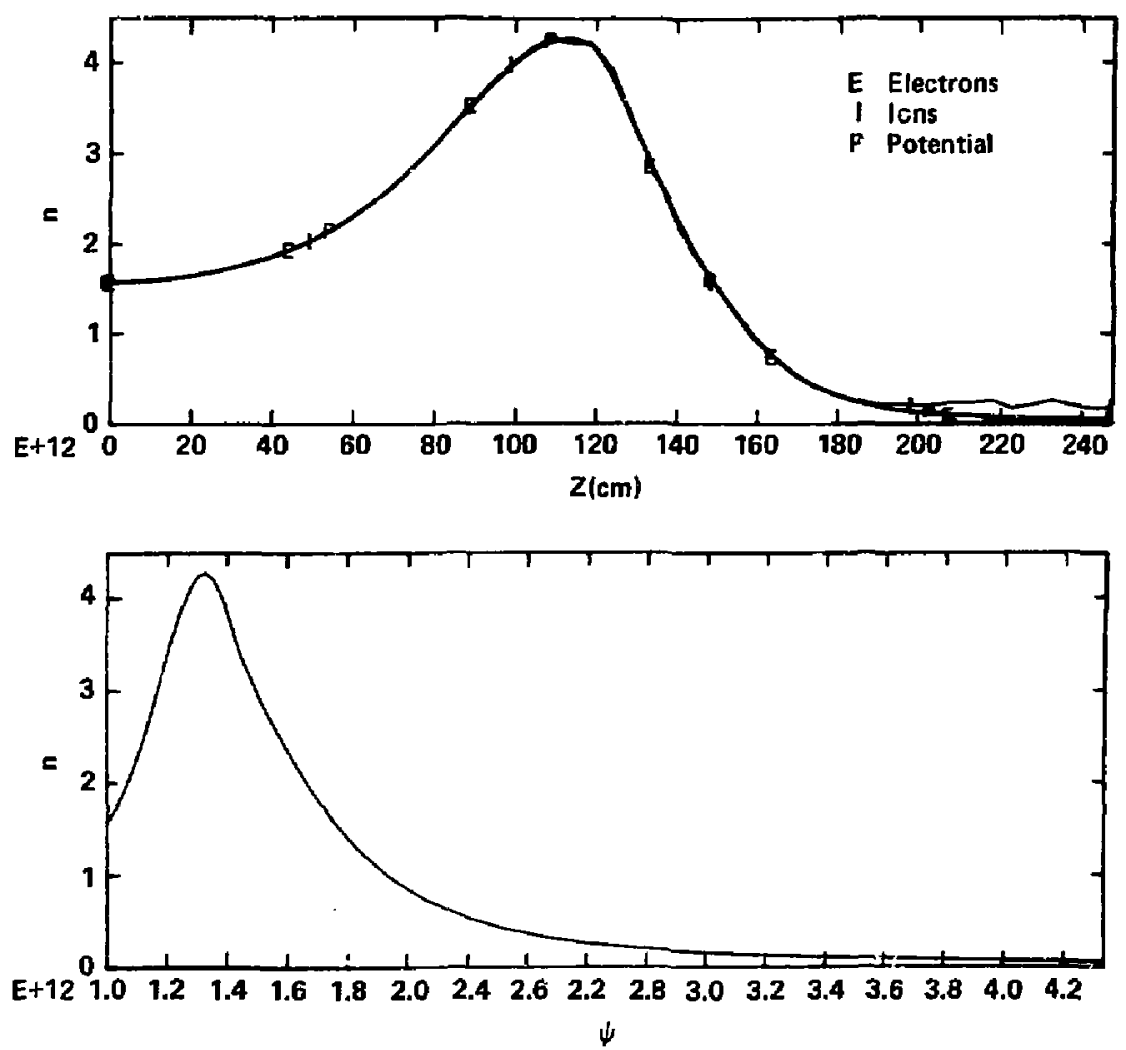

Fig. 2.4-2. Potential and density profiles obtained for sloshing-ion injection in yin-yang anchor. 


$$
\begin{aligned}
& \tau_{e e}=\frac{8.19 \times 10^{9} \mathrm{~T}_{e w}^{3 / 2}(\mathrm{keV})}{\left[n_{e w A}(a)+n_{e c A}(a)\right] \ln A_{e e}}, \\
& N_{c w}=\frac{n_{e w A}(a)}{n_{e c A}(b)}\left(\frac{T}{T_{e w}}\right)^{1 / 2} .
\end{aligned}
$$

The cold-electron-source results from the ionization of the sloshing-ion beam and the axial high-energy pump beam at the position a. Since the value of $J_{e}$ will be determined subsequently, we can find the warm-e lectron temperature that is consistent. with the neutral-bean requirements and the required potential profile.

\subsubsection{AMCHOR-CELL BEAM REQUIREMENTS}

The neutral beans injected into the barrier region in the anchor include the high-energy sloshing-ion beam, incident at $90^{\circ}$ at the 1.2-T outboard position, and an axial high-energy punp beam (HEPB). The HEPB is designed to pump out the warm trapped ions, which would otherwise build up in the potential well in the barrier. In addition, the HEPB pumps the hot sloshing ions and maintains the appropriate ratio of peak to minimum sloshing-ion density in the anchor $\left(R_{S}\right)$.

The potentially trapped ion current in the anchor and transition regions is determined using a bounce-averaged Fokker-Planck calculation ${ }^{2.15}$ with the loss boundaries and distribution functions shown in Fig. 2.4-3. The magnetically trapped current is calculated as in Ref. 2.1 with a correction factor (Devoto ${ }^{2.16}$ ) included. The magnetically trapped current then is given by

$$
I_{\mu}=\frac{q g_{b}\left(b^{*}\right) n_{j W A}^{2}\left(b^{*}\right) v_{b}^{*}}{2.5 \times 10^{10} T_{i w}^{3 / 2}\left(2 \theta^{*} / \pi\right)^{2} D},
$$

where the Devoto correction factor for the MFTF-B Axicell geometry is

$$
0 \simeq 8 \text {, }
$$

and

$$
\sin \theta *=\frac{B_{b}+B_{a}}{2 B_{m A D}} \quad\left(1+\frac{\phi_{c}}{2 T_{i n}}\right)
$$




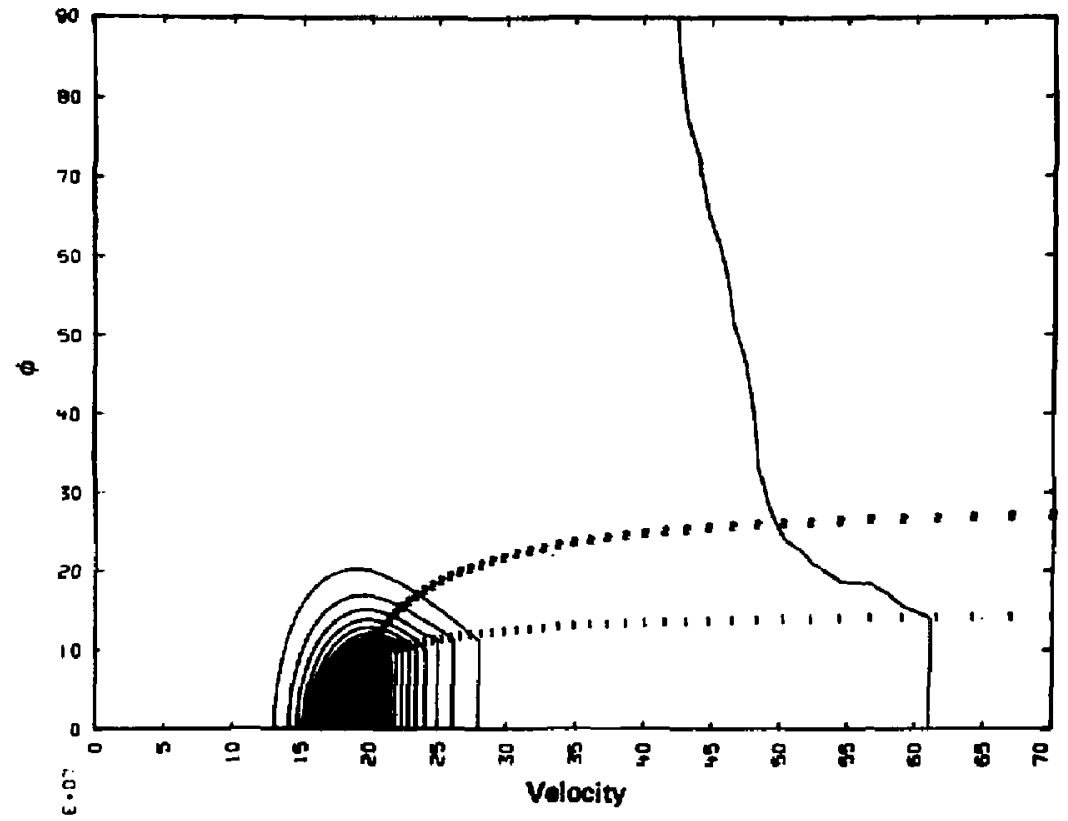

Fig. 2.4-3. Loss boundary and distribution-function contours for passing and trapped ions in the transition region. 
The position $\underline{b}^{*}$ is the point where the potential on the out soard side of the barrier rises back to the value in the central cel1; $v_{b}^{*}$ is the effective volume in this region; and $g_{b}\left(b^{*}\right)=2.75$. The value of $I_{\mu}$ is generally quite small and equal to about $10 \%$ of the potentially trapped ions. The total trapped current is then taken to be the sum of the potentially and magnetically trapped currents.

The magnitude of the HEPB is determined by the requirement that the charge-exchange pumping rate in the bottom of the barrier matches the pumping rate necessary to maintain the sloshing-ion-density distribution with the required ratio of peak density to minimum density as determined by bounce-averaged Fokker-Planck 2.17 calculations of the anchor. Since the HEPB is absorbed and also pumps in the other regions of the barrier and transition regions, the total pumping by the HEPB is about $15 \%$ of I trap. The remaining $85 \%$ is to be pumped by the passing-particle barrier beam (P2B2).

The ionization current of the sloshing-ion beam in the anchor balances the scattering losses of the hot sloshing ions and the charge-exchange losses of $f$ the HEPB. If, in addition, the one-half and one-third energy components of the sloshing beam were not confined when injected at the 1.2-tesla point, they would act as a charge-exchange pump removing the hot sloshing ions that are confined. The ionization current would then be written

$$
I_{\text {slosh }}^{(\text {ion })}=\frac{I_{\text {scat }}+I_{c x}^{\text {HEPB }}}{1-F_{2}} .
$$

Here $1^{\text {scat }}$ is the scattering-loss current obtained by Logan 2.18 from modeling fokker-Planck calculations of the scattering ?oss of the sloshing ions,

$$
I^{\text {scat }}=\frac{q n_{\text {ihA }}^{2}(b) v_{5 l o s h}}{(n \tau)_{5 l o s h}} \text {, }
$$


and

$$
\begin{aligned}
& (n \tau)_{s \operatorname{losh}}=2.23 \times 10^{10}\left(\bar{E}_{t}\right)^{3 / 2} \log _{10}\left(\frac{B_{m A 0^{\prime B}} / Q_{m}-\phi_{i n j}}{1-\frac{Q_{m}}{E_{t}}}\right) \text {, } \\
& E_{t}=\frac{\sum_{j=1,1 / 2,1 / 3}\left[\frac{\langle\sigma v\rangle_{i j}}{v_{j}} f_{j} E_{j}+\frac{\langle\sigma v\rangle_{c x j}}{v_{j}} f_{j}\left(E_{j}-E_{i n}\right)\right]}{\frac{\langle\sigma v\rangle}{v_{j j}} f_{j}}, \\
& E_{i n}=\frac{\sum_{j=1,1 / 2,1 / 3} \frac{\text { sov }_{i j}}{v_{j}} f_{j} E_{j}}{\sum_{j=1,1 / 2,1 / 2}^{\frac{\langle o v\rangle_{i j}}{v_{j}} f_{j}}} .
\end{aligned}
$$

When the one-half and one-third beam-energy components are not conf iried, the term $F_{2}$ is the ratio of the charge-exchange rate of the one-half and one-third beam-energy components to the jonization rate of the full-energy componeht of the sloshing beam. For our standard case, all the beam-energy componeryts are confined, and $F_{2}$ is zero; $I_{c x}^{H E P B}$ is the charge-exchange loss current resulting from charge-exchange interactions between the sloshing ions anH the HEPB ions as they traverse the anchor. For the reference-cas 6 parameters, the scattering loss of the sioshing ions is comparable to the charge-texchange losses of the HEPB.

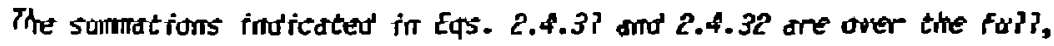
the one-half, and the one-third beam-energy components of the beam; $\langle O V\rangle_{i j}$ and <ov ${ }_{C x j}$ are the total ionization and charge-exchange rates for beam component $j$. Similarly, $E_{j}$ and $f_{j}$ are the energy and dtomic fraction for each beam component; and $v_{j}$ is the velocity of beam component $j$, corresponding to the energy $E_{j} ;\left(\phi_{m}-\phi_{i n j}\right)$ is the potential difference between the outer mirror and the point of beam injection.

The ionization current from the sloshing beam and the ionization current of the MEPB deposited in the region of the potential peak provide the cold electrons that form the cold-electron source $J_{e}$ in Eq-2.4.23. This value of $\mathrm{J}_{\mathrm{e}}$ places a limit on the maximum sloshing-ion current and HEPB current 
that can be used in the axicell. When $J_{e}$ becones too large, the model Eq. 2.4.22 only has solutions for $T_{\text {ewA }}>\delta \phi_{a}$, a regime where the model becomes inaccurate. In practice, the value of $\mathrm{J}_{e}$ is 1 imited to several microamps per cubic centiaeter.

\subsubsection{MICRONAVE PONER REQUIREMENTS}

The microwave power supplied to the potential peak region a and the thermal barrier region $\underline{b}$ in the axicell is calculated in a maner similar to that in Ref. 2.11. The power per unft volume at a is given by

$$
P_{a}=J_{\text {in }} \frac{B_{b}}{B_{a}}\left(T_{e w A}-T_{e c A}\right)+J_{e}\left(\delta \phi_{a}+\frac{B_{b}}{B_{a}} T_{e w A}\right)+\frac{1}{2} J_{i n}\left(T_{e w A}-T_{e C A}\right) \text {. }
$$

This is the so-called "weak ECRH" 1imit of Ref. 2-11, where

$$
\mathrm{j}_{\text {in }}=\frac{n_{\text {ecA }}(\mathrm{b}) n_{\text {ewA }}(\mathrm{a})}{(n \tau)}\left(\frac{T_{\text {ewA }}}{T_{\text {ecA }}}\right)^{1 / 2} e^{\delta \phi_{a} / T_{\text {ewA }}}
$$

and

$$
(n \tau)=\frac{\sqrt{\pi}}{4}\left(1+\frac{B_{b}}{B_{a}}\right) n_{\text {ewA }}(a) \tau_{\text {ee }}\left(e^{\delta \phi_{a} / T_{\text {ewA }}}-1\right)
$$

The dominant term in Eq. 2.4.33 is the one involving $\mathrm{J}_{e}$. Hence, the power at a is controlled by the magnitude of the cold-electron source at a.

The microwave power needed to produce the hot electrons is modeled as the sum of scattering and synchrotron radiation losses. The scattering loss per unit volume is

$$
P_{\text {SCATT }}=\frac{q n_{i A}^{2}(b)\left(1-F_{e c}\right)\left(E_{e h A} / 2\right)}{n T},
$$

where

$$
n \tau=1.65 \times 10^{8} E_{e h A}^{3 / 2} \log \left(R_{e f f}\right)
$$

The numerical coefficient of Eq. 2,4.39 is law by comparison with recent electron Fokker-Planck calculations in this geometry, ${ }^{2.19}$ which are best fitted with the coefficient $2.8 \times 10^{8}$. However, those calcuiarions include 
only the hot-electron population and neglect drag effects between the hot electrons and the other electron populations whose spatial distributions overlap. Depending on the details of the spatial distribution, the drag effects might add as much as 50 to $60 \%$ to the power estimate for the hot electrons at $\underline{b}$. Thus, retaining the lower coefficient in Eq. 2.4.38 is tantamount to correcting for these drag effects, giving a more realistic modeling of the total microwave power needed at $\underline{b}$. However, because sone of this drag power is transferred to the warm electrons at $a$, we would expect the power estimate $P_{a}$ given by Eq. 2.4.33 to be higher than necessary.

The value of $R_{\text {eff }}$ is taken as the smaller of $R_{\text {eff }}$ for losses to the inner-mirror point or the outer-mirror point, where

$$
\begin{aligned}
& R_{\text {eff }} \text { (inner) }=\frac{B_{m A 1} / B_{b}\left(1-B_{b}\right)^{1 / 2}}{1+E_{\text {ehA }}}, \\
& R_{\text {eff }} \text { (outer) }=B_{m A d} / B_{b}\left(1-B_{b}\right)^{1 / 2} .
\end{aligned}
$$

Here we assume that the potential at the outer-mirror point is the same as that at the botton of the themal barrier. In general, because $B_{m A i}$ is so much larger than $B_{\text {mAd }}, R_{\text {eff }}=R_{\text {eff }}$ (outer).

\subsubsection{CENTRAL-CELL ION-PARTICLE AND EMERGY BALANCE}

This model assumes all the central-cell ions are thermal ions with a

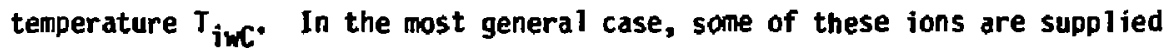
by ionization of gas and by a neutral beam in the central cell. In addition, some of the ions produced by charge exchange and ionization of the passing-particle barrier bean (P282) in the transition region become trapped in the central cell. A third source of ions for the central cell is the ionization current from the axicells adjacent to the central cell. The balance equation for the thermal ions then becomes

$$
\begin{aligned}
& I_{i}^{\text {loss }}+2 I^{\text {trap }}+2 I_{\text {abs }}^{P 2 B 2} f_{C X}^{P 2 B 2} \\
&=I_{C \text { ion }}^{\text {gas }}+2 I^{P 2 B 2} f_{\text {trap }}^{P 2 B 2}+I_{C} \text { ion }+2 I_{X} \text { ion } .
\end{aligned}
$$


The axial loss current $1_{i}^{\text {loss }}$ of the warm central-cell ions is given by

$$
I_{j}^{l 0 s s}=q \frac{n_{j w C}^{2} v_{c}}{(n \tau)_{j w C}},
$$

where $(n \tau){ }_{i w C}$ is the ion Pastukhov confinement parameter for the warm ions; $f_{\text {trap }}^{P 2 B 2}$ is the fraction of ions from the passing-particle barrier bean that are eventualiy confined in the central ce11; $f_{C X}^{P 2 B 2}$ is the fraction of the absorbed $P 2 B 2$ that charge-exchanges on the passing ions in the transition region; I trap is the current of ions from the central cell that traps in the transition and anchor regions; $\mathrm{I}_{\mathrm{P} 2 \mathrm{bS}}$ is the total passing-particle barrier-bean current absorbed in the transition region; I $c$ is ion" $I_{c}$ ion, and $I_{x}$ ion are the ionization currents for the central-cell gas and neutral beams and the axicell neutral beam, respectively. The factors of two in Eq. 2.4.40 account for the particle losses and inputs from each end of the system.

The ion-energy equation is obtajned by balancing the energy carried out by each ion with the energy input of each source jon. The difference in potential between the point of production of the jon and the central cell is also considered in the energy bookkeeping. The equation is

$$
\begin{aligned}
& I_{C \text { ion }} f_{i i} E_{\text {trap }}+P_{C}^{I C R H}+2 I_{a b s}^{P 2 B 2}\left(E_{P 2 B 2}-\delta \phi_{L}+\Delta \phi_{p c}\right) f_{\text {trap }}^{P 2 B 2} \\
& +2 I_{X \text { ion }}\left(E_{\text {loss }}+\Delta \phi_{p c}\right) \\
& +2 I_{X \text { ion }}\left(E_{\text {loss }}+\Delta \phi_{p c}-E_{i c}\right) \frac{\text { <ovs }}{\text { covs }} \frac{n_{i X}^{\text {pass }}}{n_{j X}} \\
& =1_{i}^{\text {loss }}\left(T_{i w C}+\phi_{C}\right)+2 I^{\operatorname{trap}} E_{i w C}
\end{aligned}
$$

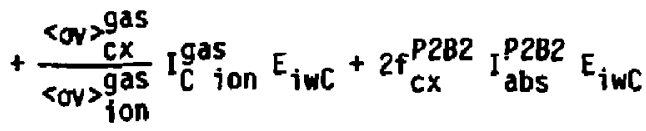

$$
\begin{aligned}
& +\frac{q n_{j w r}^{2} V_{c}}{(n \tau)_{d r a g}}\left(E_{i w C}-E_{e c}\right)
\end{aligned}
$$


where $E_{\text {trap }}$ is tine mean beam energy trapped per beam neutral ionized, and $f_{i j}$ is the fraction of this energy deposited in the ions. The values of $E_{t r a p}$ and $f_{i j}$ are calculated with the formulism of Ref. 2.12 using the atomic fractions,

$$
\begin{aligned}
& f_{1}=0.833, \\
& f_{1 / 2}=0.075, \\
& f_{1 / 3}=0.092,
\end{aligned}
$$

calculated for the 80-kV neutral beams with a 90:5:5 molecular mix in the arc chamber.

The absorbed fraction of the P282 supplies to the central cell an energy per particie, $\left(E_{P 2 B 2}-\delta \phi_{L}+\Delta \phi_{p C}\right)$, where $E_{P 2 B 2}$ is the average beam energy and $\delta \phi_{L}$ is the average potential drop between the potential peak in the axicell and the location where the P2B2 ion is born. The neutral beam in the axicell contributes energy equal to its loss energy (calculated in Eqs. 2.4.8 and 2.4.9 from the axicell plus the change in potential $\Delta \varphi_{\mathrm{pc}}$. Moreover, because the passing ions from the central cell can charge-exchange with the axicell beam, there is a second term in the energy balance that depends on the axicell jonization current.

The loss terms in the energy balance include the axial energy carried out by the ions, the energy lost by ion trapping in the transition and anchor regions, the charge exchange losses by passing ions in the transition and by interactions with gas in the central cell, and also the energy jost by electron drag.

In the general problem of particle and energy balance, we solve (2.4.40) for the gas current (for a given ion-confining potential) and substitute into Eq. 2.4.42. This gives the $r f$ power, $P I C R H$, as a function of the central-cell-beam ionization current, $I_{C}$ ion or vice versa. However, to find the optimum $(n \tau)_{i w c}$ for the system, we solve the balance equations with the restriction that ${ }_{\mathbf{C}} \mathbf{g}$ ion $=0$. In addition, we set the central-cell-beam current to zero and obtain the maximum $(n \tau)_{j w C}$ that can be achieved with the other given beam inputs. With the gas and central-cell beams set to zero, we solve Eq. 2.4.40 for I loss (and hence $\phi_{C}$ ) to give particle balance, and then use $E q .2 .4 .42$ to solve for the value of $p^{I C R H}$, which gives energy balance. 


\subsubsection{CENTRAL-CELL ELECTRON-PARTICLE AND ENERGY BALANCE}

The electron-particle losses are all assumed to be axial losses, so that the electron-loss current is written as

$$
I_{e}^{\text {Loss }}=\frac{q n_{e C}^{2} v_{c}}{(n \tau)_{e C}}
$$

Here, $(n \tau)_{e c}$ is the Pastukhov confinement for electrons in the confining potential $\phi_{\mathrm{e}}$.

The electron sources result from the ionization of the gas, the central cell and axicell beams, as well as the HEPB, Р2B2 and sloshing-ion beams in the anchor and transition regions. In addition, a source of secondary electrons $I_{s e c}$ flow in from the end walls. This current is given in terms of the primary electron loss current by 2.20

$$
I^{\text {sec }}=\lambda I_{e}^{\text {Loss }}
$$

where $\lambda$ is the secondary-emission coefficient. In terms of the total ion lass out the ends of the system, $I_{\text {tot }}$,

$$
\lambda=\frac{I^{\sec }}{I^{\text {sec }}+I^{\text {tot }}} \text {, }
$$

where

$$
I^{\text {tot }}=\frac{q n_{i W C}^{2} V_{C}}{(n T)_{i W C}}+2\left(I_{A \text { ion }}^{\text {slosh }}+I_{\text {ion }}^{H E P B}+I_{\text {ion }}^{P 2 B 2}+I_{X \text { ion }}\right)
$$

The electron-particle balance then becomes

$$
I^{\text {sec }}+I_{C \text { ion }}+I^{\text {gas }}+2\left(I_{A \text { ion }}^{\text {slosh }}+I_{\text {ion }}^{\text {HEPB }}+I_{\text {ion }}^{P 2 B 2}+I_{X \text { ion }}\right)=\frac{q n_{e C}^{2} V_{C}}{(n \tau)_{e C}} .
$$

In the centraj-cell electron energy balance, the position of ionization is taken into consideration. The potential change between this point and the central cell is then added or subtracted appropriately, depending on whether the electron is a net energy source or sink to the central-cell electron population. The rate equation then is 


$$
\begin{aligned}
& \frac{q r_{i w C}^{2} V_{c}}{(n \tau))_{d r a g}}\left(E_{i w C}-E_{e C}\right)+I^{s e c} \phi_{e}+P_{C}+2\left(P_{a}+P_{b}\right) \\
& +I_{C \text { ion }}\left(1-f_{i i}\right) E_{\text {trap }}+2\left[I_{\text {ion }}^{H E P B} \bar{\phi}+I_{i \text { on }}^{P 2 B 2}\left(\delta \phi_{L}-\Delta \phi_{p c}\right)\right] \\
& =\frac{q n_{e c}^{2} V_{c}}{\langle n \tau\rangle_{e C}}\left(\phi_{e}+T_{e c}\right)+2 I_{A \text { ion }}^{5 \operatorname{losh}} \phi_{c}+2 I_{X \text { ion }} \Delta \phi_{D C} .
\end{aligned}
$$

In this model, the secondary electrons carry in the energy $\phi_{e}$ obtained from the potential drop from the wall to the central cell. Me assume that all the microwave power that produces the thermal barrier at $\underline{b}$ and the potential peak at $\underline{a}$ eventually ends up in the central ce11. This tends to overestimate the central-cell power input, since some of the hot, magnetically conf ined electrons that form the thermal barrier are lost to the end walls, rather than to the central cell.

The term $P_{C}$ al lows us to introduce power for direct electron heating of the central-cell electrons. A portion $\left(1-f_{i j}\right)$ of the trapped energy from the central-cell neutral beams goes directly into the electrons. The electrons produced by ionization of the HEPB and P2B2 in the anchor and transition carry in energy obtained from their change in potential in entering the central cell. The electrons produced by ionization of the sloshing-ion beam in the anchor must climb a potential approximately equal to $\phi_{c}$ to reach the central cell, and hence appear as an energy-loss term in the central cell. Similarly, the axicell electrons must climb the potential $\Delta \Phi_{p c}$ to reach the central cell.

The solution of the two rate Eqs. 2.4.46 and 2.4.47 gives us the secondary-electron current and a new value for the electron-conf ining potential $\phi_{e^{*}}$ Recall that we initially set $\phi_{e}$ by requiring that the ion and electron Pastukhov-conf inement parafeters be equal. Now when we introduce the secondary electrons, the electron-conf inement parameter and $\phi_{e}$ are appropriately smaller. This new value of $\phi_{e}$ is then used to recalculate the barrier parameters, beam requirements, and microwave power needs. Using the electron-balance equations with these revised paraneters gives the next iteration value for $\phi_{e}$. Experience has shown that $\phi_{e}$ converges to better than $1 \%$ in one iteration. 
Along with the secondary-electron current, we can calculate the secondary-emission coefficient using $\mathrm{Eq}$. 2.4 .45 and compare it to the value of the coefficient when the secondary-electron current is assumed to be limited by space-charge effects. The space-charge 1 imited value of the secondary-emission coefficient has been approximated by Logan 2.21 as

$$
\lambda_{s C l}=\left[0.15 \ln \left(\frac{T_{e c}}{\phi_{e}+\phi_{c}+T_{i w}}\right)+0.656\right]^{1 / 4} .
$$

If $\lambda<\lambda_{\text {sec }}$, then clearly sone suppression schene would have to be invoked to get $I_{\text {sec }}$ below the space-charge limited value.

The ECRH power necessary to achieve the electron-particle and energy balance is larger than the ECRH power required to maintain the hot-electron and warm-electron populations against the mainly classical loss processes nodeled by Eqs. 2.4.33 to 2.4.39. While this extra power cannot be directly fed to the electrons in the central-cell region, the energy can be supplied to the central-cell electrons by an appropriately designed microwave system in the barrier region. The design would be flexible enough so that we would supply the power necessary to maintain the hot and warm electrons as well as heat sone of the cold electron's that pass to the centrai cell. This extra power capability in the barrier region could also be used to supply more energy to the hot and warm electrons in the event that non-classical processes enhance the electron losses in the barrier region.

\section{REFEREMCES}

2.12. D. E. Baldwin, B. G. Logan, and T. C. Simonen, Eds., Physics Basis for MFTF-B, LLNL Report, UC1D-18496, Pt. 1 (January 1980).

2.13. R. H. Bulmer, private communication, April 1982.

2.14. M. E. Rensink, private communication, February 1982.

2.15. A. H. Futch and G. H. Leppelmeier, private communication, Apri 1982.

2.16. R. S. Devoto, Memrorandun, January 1982.

2.17. M. E. Rensink, private communication, April 1982.

2.18. B. G. Logan, private comunication, December 1981.

2.19. J. W. Shearer, private communication, February 1982. 
2.20. D. Porter, Effect of Gas Recycling and Secondary Emission on the Axial Power Flow in an Open-Ended Device, LLNL Report, UCRL-85847 (June 1981).

2.21. F. H. Coensgen, T. C. Simonen, A. K. Chargin, and B. G. Logan, TMN-U Major Project Proposal, LLNL Report, LLL-Prop-172 (Apri1 1980). 


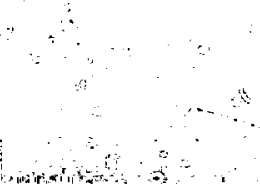




\title{
APPENDIX A \\ Analytic equilibria with quadrupole symmetry in the paraxial limit
}

\author{
L. O. Pearlstein, T. B. Kaiser, and William A. Newcomb \\ Laurince Livermore National Labaratory. Unitersity of California Liremore. Colifomia 04550 \\ (Recerived 15 December 1980; accepied il March 1981]
}

Mirfus equilibria for arbitrary mirror ratio and nun-iube eccentricity are obiatned to leading order in the plasma pressure (beta expansion) in the paraxial limit jaxal scale lengths long compared woth radral scale lengths!. The solutions are given in terms of quadrutures over known functions. The theory ts applied to a tandem-mirror configuration

\section{INTRODUCTION}

Adiabatic confinement in conventional mirrors is controlled primarily by the $z$ component of the axtsymmetrlc part of the applted magnetic fleld. How ever, to create a stable conflguration (Interchange mothon being the problem) it is necessary to generate a "minimum-B well." This is usually done by adding a quadrupole (sometimes even a higher order multipole) component to the main fleid. This addition, of course, makes the gulding-center equilibrium problem threedimensional. In general, the solution of this equitibrium get of equations requires large sophisticated codes and state-of-the-art digital computers. ${ }^{1,2}$ Even then results tend to be relatively crude, because of the limitations of computer memory capacity and speed. However, because the symmetry breaking fleid is weak compared with the main axisy mmetric field, and be cause the radius of curvature of the main axisymmetric field is large compared with other characteristic lengths, an important simplification can be made. This simplication in the equllibrium model has been calied the "long-thin" or paraxial ayjroximation. Specifically, if we identily the small parameter $A$ as the ratio of radial scale length to axial scale length, then it can be systematically seen that: the perpendicuLar components of the magnetic field are of order $\lambda$ compared with the main $z$ component; the radius of curvature is of order $\lambda^{-2}$ compared with the radial scale length; and the local parallel current is of order $\lambda$ compared with the local perpendicular current.

Using this orderlng, we generate a reduced set of equations in flux coordinates for the equllibrium in Sec. II. These equations are nonetheless a formidable set, and solvtng them is still an extensive numerical problem, even though they have the further advantage that $z$ is a time-like coordinate in the partial differentlai equation determining the flux-line geometry. That is, the equation can be formally jntegrated from one plane of constant $z$ to the next and then Information from the original plane can be discarded. Hence, in principle, the required computer storage becomes that of a two-dimensionat problem. Unfortunately, the axlal boundary conditions come from both ends.

To make further progress, we look for a self-consistent equilibrium to first order in the plasma pressure. That is, we expand the equilibrium equattons treating the ratlo of plasma to magnetic field pressure (beta) as a small expansion parameter, but large com pared with $\lambda^{7}$. This expanstion is carried out for quadrupole symmetry in Secs. III and IV; in Stc. III we develop the general perturbation scheme and in Sec. IV we obtain explicit solutions. The solutions are given as guadratures over known yacuum-magnetic-lield quantilies (functions of $z$ ) and over prescribed pressure profiles (functions of mod- $B$ and the principal flux). We emphasize that, given the ratural smaltness parameter $\lambda$, we need only assume low beta to obtain analy tic solutions; the mircor ratio minus one and llux-tube eccentricity are arbitrary differing from zero by terms of order unity.

Section $\mathbf{V}$ gives our results. There are two features of the equilibrium which we indicate here. We lind, as Stupakov did, ${ }^{3}$ that there is a breakdown in the pertur. bation expansion, ap apparent bifurcation point when the flute instability criterion ts marginal. This we beHeve to be an interesting point: whether there is, in fact, another equilibrium we are not able to say at this time. The other feature, which is very favorable, is that the self-consistent equilibrium reduces the strong ellipticity in the transition regions of tandem mirrors. This is coatrary to the results in Ret. 3 . The reasons for this difference are discussed in Sec. $V$.

The Heaviside-Lorentz system of units, in which $\epsilon_{0}$ $=\mu_{0}=1$, is used throughout.

\section{EQUILIBRIUM EQUATIONS IN THE PAFAXIAL APPROXIMATION}

Our starting point is the general tensor-pressure magnetostatic equilibrium conditions,"

$$
(\nabla P)_{4}=Q K_{1}
$$

and

$$
\frac{d P}{d B}=B \frac{d}{d B}\left(\frac{Q}{B}\right)
$$

where

$$
\begin{aligned}
& P=B^{2} / 2+p_{1}, \\
& Q=B^{2}+p_{1}-p_{0},
\end{aligned}
$$

$B$ is the magnetic flux density, $x$ is the curvature vector, and $p_{2}$ and $p_{0}$ are the pressure components perpendicular and parallel to $B$. The derivatives $d / d B$ tn Eq. (2) should be undersiood as directional derivatives 
in the parallel direction, i.e.,

$$
\frac{d}{d B}=(B \cdot \nabla B)^{-1} B \cdot \nabla .
$$

Here and elsewhere, $|\mathbf{g}|$ is abbreviated as $B$ and $\hat{b}$ de notes the unit vector $p$ arallel to $B$. Then

$$
x=\hat{b} \cdot \nabla \hat{b}=-\hat{b} \times(\nabla \times \hat{b}) \text {. }
$$

Apply the operator $b \cdot \nabla \times$ to Eq. (6) to obtair

$$
\hat{b} \cdot \nabla \times x=B b+\nabla(i / B),
$$

where $i$, the parallel current per unit magnetic flux, is

$$
i=b \cdot \nabla \times b \text {. }
$$

Next, apply the same operator to Eq. (1) and obtadn

$$
b \cdot \nabla \times Q x=-1 B b \cdot \nabla(Q / B) \text {. }
$$

Then, with Eq. (7) we have the usual parallel current equation

$$
\hat{b} \cdot K \times \nabla Q=b^{\circ} b \cdot \nabla\left(Q t / \nabla^{*}\right) .
$$

These equations, given the requisite condillons for well-posedness' (or local stablity)

$$
\frac{d P}{d B}>0 \text { (mirror mode) }
$$

and

$Q>0$ (Itrehase mode),

determine the equilibrium.

We now introduce nux coorcinated $\alpha, \beta$ with the property that

$$
\mathbf{B}=\boldsymbol{\nabla} \boldsymbol{\alpha} \times \boldsymbol{\nabla} \boldsymbol{\beta},
$$

and

$$
\hat{b} \cdot \nabla \boldsymbol{a}=\boldsymbol{b} \cdot \nabla \boldsymbol{\beta}=\mathbf{0} .
$$

The pressure distribution is assumed to be of the spectal form

$$
p_{1,1}=p_{1,4}(a, B) \text {. }
$$

That is, aside from its dependence on $B$, it is a funetion of only one tux coordinate, the sn-called principal flux coordinate. We will say more about this later.

From Eq. (2), (remember $d / d B=\partial / a B$ ) a $_{\text {] }}$ we obtain the usual relationship between $p_{2}$ and $p_{n}$ :

$$
p_{1}=-B^{*} \frac{d}{d B}\left(\frac{p_{11}}{B}\right)
$$

so that only $P_{x}(\alpha, B)$ need be spectfied.

Henceforth, we assume a large-aspect-ratlo system so that we make the paraxial (or long-thin) approximation. That is, lengths in the $x, y$ plane are small (of order $x$ ) compared with the axial scale length (In the $z$ direction), and the field components $B_{r}, B_{\text {, are simllar - }}$ ly small, of order $A$, compared with $B_{,}$and $B_{\text {, van - }}$ ishes nowhere. In all calculations hencelorth, all terms of relative order $\lambda^{2}$ are systematically dropped. If we take the unit of length to be on the order of the transirerse scale letigth, then all gradients in the $z$ direction are of order $\lambda$, and because of this $*$ is of or - der $\lambda^{2}$. With $d s$ denoting the element of arc length along a llux line (constant $\alpha, \beta$ ), we have

$$
d s=d z \text {. }
$$

Furthermore, to this order

$$
B_{*}=B \text {. }
$$

From Eq. (1) it follows that

$$
\bar{F}=P(z)=\frac{1}{2} B_{0}^{2}(z),
$$

where $B_{4}$ denotes the vacuum field. Given $P(z)$ and $P_{11}(a, B)$, only a functional inversion is requlred to determine $B, P_{n}, P_{1}$ ag lunctions of $a, z$. All are indepen dert of 8 .

To complete the deseription of the equilibrium requires a determination of the coordinate iransformation $(\alpha, \beta, z)$ to $(x, y, z)$. It is thus neceasary to determine the llux-line geometry $x(\alpha, \beta, z), y(\alpha, \beta, z)$.

One equation in the two unknowns $x, y$ is immediately obtainable from the definition of $\sigma, \beta$ as Rux coordinates, viz.,

$$
\frac{\partial(x, y)}{\partial(\alpha, \beta)}=\frac{1}{B}
$$

(Bee Appendlx $A$ ), where $B$ !a a known furction of $a$ and $z$. (The standard notation 16 used be re to denote the Jacobian of $x$ and $y$ with respect 10 a and $\beta_{\text {.) }}$ To obtain a second relation, we first define the covariant curvature componente A (normal curvalure), 10 (geodesic curvatirsi 50 that

$$
\Delta=\pi \nabla \sigma+10 \nabla \theta .
$$

Thus (primes are used to denote partial derivatives with respect to $z$ for fixed $a, \theta)$,

$$
\boldsymbol{R}=x^{*} \frac{\partial x}{\partial a}+y^{e} \frac{\partial y}{\partial a},
$$

and

$$
10=x^{n} \frac{\partial x}{\partial \theta}+y^{n} \frac{\partial y}{\partial \theta} .
$$

Furthermore, the deltinition Ea. 181 reduces to

$$
i=\theta\left(\frac{\partial\left(x^{\prime}, x\right)}{\partial(\alpha, \beta)}+\frac{\partial\left(y^{\prime}, y\right)}{\partial(\alpha, \beta)}\right)
$$

(see Appendix A), and finally, from Eq. (9), we obtain the desired second equation in $x$ and $y$ of the form

$$
\omega \frac{\partial Q}{\partial a}=-B\left(\frac{Q i}{B^{9}}\right)^{\prime},
$$

where again $Q$ is a known function of $a, z$. (The partial aQ/za is taken for fixed z.) Presumably Eqs. (17)(22), in cunjunction with the appropriate boundary conditions, would suffice to determine the unknown coor dinates $x(\alpha, \beta, z)$ and $y(\alpha, \beta, z)$. In the next section we linearize these equations about their vacuum values to obtain the equations which define the flux line geometry to leading order in beta (the ratio of the plasma energy density to fleld energy density). 


\section{FLUX-iINE GEOMETRY (LEADING ORDER (N BETA)}

To lowest order the flux-line geometry is determined by the vactum fields. For quadrupole symmetry in the paraxial appruximation, the field line trajectories can be written

$$
\begin{aligned}
& x_{v}=\sigma(z) x_{0}, \\
& y_{0}=\tau(z) y_{0},
\end{aligned}
$$

where $\sigma$ and $\tau$ satisfy

$$
\sigma \tau=\left(B_{0}\right)_{1} / s_{,}(z) \text {. }
$$

and where $x_{0}$ and $y_{a}$ are the coordinates of the fletd lines at the mftplane $(z=0)$ of the conflguration, and $B_{0}=B(0)$. In what follows, we assume the type of symmetry defined by the two conditions

$$
B_{w}(z)=U_{w}(-z) \text {. }
$$

and

$$
\sigma(z)=\tau(-z),
$$

This, of course, ts the symmetry of the standard minimum-13 mirror (with the lang rotated through an angle of $90^{4}$ relative to each other) and of tandem mir rors such as TMX, Ambol, and Phaedrus. We also jdentify the transverse vacuum-field components

$$
\begin{aligned}
& B_{x}=x^{\prime} B=\tau_{0} B_{0} \sigma^{\prime}, \\
& B_{y}=y^{\prime} B=y_{0} B_{0} \tau^{\prime},
\end{aligned}
$$

where the first equality is valid for any fleld in the paraxial limit, and the second is valid for the vacuum field only. The components $B_{x}, B_{y}$, as such, will not appear explicitiy in further calculations but are written down here for completeness.

Next, we jdentify the principal flux coordinate to this order. Fram the parallel-current constraint (22), we force

$$
\int_{-L}^{L} \frac{d z}{B} \frac{\partial Q}{d \alpha} 50=0
$$

in order that the parallel current whll vanish at the ends of the devlce (a necessary condition for 2 plasma to be canjined and jsalated frow the surrounding anyirosment). Since

$$
\frac{\partial Q}{\partial \alpha}=-2 \frac{\partial p}{\partial \alpha},
$$

where $p=\left(p_{1}+p_{11}\right) / 2$, is already of order beta, we need only the vacuum values of the remaining quantities in

(29). If we now make the identification of

$$
\begin{aligned}
& \alpha=\psi=r^{2} B_{v}(0) / 2, j^{2}=x_{0}^{2}+y_{0}^{2}, \\
& \theta=\theta=\tan ^{-1}\left(y_{0} / x_{0}\right),
\end{aligned}
$$

we see that constralnt (29) is satisfled by symmetry, since

$$
50=-r^{2}\left(00^{*}-\tau \tau^{n}\right) \sin \theta \cos \theta
$$

is antisymmetric. Thus, the leading -order expression for the parallel current is

$$
i=r^{2} \sin 2 \theta \int_{r}^{L} \frac{d z^{\prime}}{B_{v}}\left(00^{\prime \prime}-\tau \tau^{-1}\right) \frac{\partial P}{\partial \psi} .
$$

To next order, we set

$$
\begin{aligned}
& x=x_{0}+\varepsilon, \\
& y=y_{7}+\eta,
\end{aligned}
$$

and obtain, after linearizing Eq. (I7),

$$
\frac{a(\xi, y)}{\partial(\downarrow, \partial)}+\frac{\partial(x, \eta)}{\partial(\downarrow, \theta)}=\delta\left(\frac{1}{B}\right)
$$

but, now let us change coordinates from $\psi, \theta$ to $x_{0}, \gamma_{0}$. Using Eq. (3), we obtain

$$
\tau \frac{\partial E}{\partial x_{0}}+\sigma \frac{\partial \eta}{\partial y_{0}}=\frac{B_{0}}{B^{3}}\left(p_{1}-6 P\right) .
$$

In Eq. (37) $\delta P$ is the change in the vacuum solution due to the presence of plasma currents. If there is a boundary where the flux is I lxed la perfectly conducting shell at some boundary denoted by $b+6$, then

$$
s p=\frac{\int_{0}^{*} d d^{*} p_{1} / B^{3}}{r_{0}^{*} d \omega^{*} / B^{3}} .
$$

Honcelorth, all quantities refer to their vacuum values (in the absence of plasma) unless otherwise stated. Since the right-hand side of Eq. (37) is independent al 6, we can immediately construct the general solution to this equation, viz.,

$$
\begin{aligned}
& \xi=k \sigma x_{0}+\sigma \frac{\partial \phi}{\partial y_{0}}, \\
& \eta=k \tau y_{0}-\tau \frac{\partial \phi}{\partial x_{0}},
\end{aligned}
$$

where, at this stage,

$$
\phi=\phi\left(x_{0}, y_{0}, z\right)
$$

is an arbitrary stream function, and the particular solution satisfies

$$
2 \frac{B_{0}}{B} \frac{\partial}{\partial \psi}(k \psi)=\frac{B_{0}}{B^{3}}\left(D_{\perp}-\delta P\right)_{2}
$$

so that

$$
k=\frac{1}{2 \psi} \int_{0}^{\infty} d \psi^{\prime} \frac{p_{L}-5 P}{B^{2}} .
$$

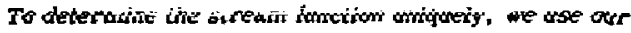
second equation tor the fiux-line coordinates, Eq. (22), and the detinition of $i, E q$. (21). Linearizing these equations generates

$$
\frac{\partial\left(x^{\prime}, \xi\right)}{\partial(\psi, \theta)}+\frac{\partial\left(y^{\prime}, \eta\right)}{\partial(\psi, \theta)}+\frac{\partial\left(x, \xi^{\prime}\right)}{\partial(\psi, \theta)}+\frac{\partial\left(y, \eta^{\prime}\right)}{\partial(\psi, \theta)}=\frac{i}{B} .
$$

Again, let us put it in terms of $x_{0}, y_{0}$ :

$$
\sigma^{\prime} \frac{\partial \xi}{\partial y_{0}}-\sigma \frac{\partial \xi^{\prime}}{\partial y_{0}}-\tau^{\prime} \frac{\partial \eta}{\partial x_{0}}+\tau \frac{\partial \eta^{\prime}}{\partial x_{0}}=i \frac{B_{0}}{B} \text {. }
$$

If we now substitute Eqs. (34), (39), and (40), we finally obtain

$$
T^{2} \frac{\partial^{2} \phi^{\prime}}{\partial x_{0}^{2}}+\sigma^{2} \frac{\partial^{2} \phi^{\prime}}{\partial y_{0}^{z}}=2 \sin 2 \theta \psi \frac{\partial}{\partial \psi} S(\psi, z),
$$

with

$S(\Psi, z)=\frac{1}{B} \int_{.}^{L} \frac{d z^{\prime}}{B} p\left(0 \sigma^{\prime \prime}-\tau \tau^{\prime \prime}\right)-\frac{1}{2}\left(\sigma^{2}-\tau^{2}\right) k^{\prime}$. 
At this point, several remarks are in order. First, all field quantities refer to their vacuum values. Second, Eq. (45) is an elliptic equation for $\phi^{\prime}\left(x_{0}, \xi_{0,} z\right)$ in which the coefficients are functions only of $z$ (f.e., only of a parameter, at this level). Consequent 45 , to solve for $\phi$ we first solve Eq. (45) subject to the laleral boundary conditlon that $\phi^{\prime}$ vanish at some $\psi=\psi^{*}$ for all $z$ and $\theta$. We then construet

$$
\phi\left(x_{0}, y_{0}, z\right)=\int_{0}^{\prime} \phi^{\prime}\left(x_{0}, y_{0}, z^{\prime}\right) d z^{\prime}+\phi_{0}\left(x_{0}, y_{0}\right) \text {. }
$$

That is, having solved for $\phi^{\prime}$, we know to within an arbitrary function of $x_{a} y_{a}$ for equivalently, of the nux coordinates).

To determine $\phi_{0}$ we demand that the parallel current still vanish at the ends to this order in the fux-line geometry. Thus, we once again apply Eq. (22) to that

$$
\int_{-L}^{L} d z \cos \left(\frac{1}{B} \frac{\partial Q}{\partial \psi}\right)+\int_{-L}^{L} d z(010) \frac{1}{B} \frac{\partial Q}{\partial \psi}=0 \text {. }
$$

Again, the first term vanishes by symmetry. Thas, we need only compute the correction to the geodeslc curvature

$$
800=\xi^{\mu} \frac{\partial x}{\partial \theta}+x^{m} \frac{\partial \xi}{\partial \theta}+\eta^{m} \frac{\partial y}{\partial \theta}+y^{*} \frac{\partial \eta}{\partial \theta} .
$$

Let us arite this $28810_{1}+6 \% 0_{1}$, श्रherein the two terms represent the contributions of $k$ and $\phi$ in the formulas for $\xi$ and $\eta$. We first have

$$
6 \mathrm{FO}_{2}=x_{0} y_{0}\left[-(0 k)^{n} \mathrm{O}+(T k)^{x_{T}}-k 00^{n}+k T T^{*}\right] \text {. }
$$

Now, since $6 \mathrm{FO}_{2}$ is an odd funetion of $x_{\text {, it too does not }}$ contribute to Eq. (48). Finally, the remaining piece is

$$
\begin{aligned}
\delta \omega_{2}= & -\sigma y_{0}\left(\sigma \frac{\partial \phi}{\partial y_{0}}\right)^{\prime \prime}+\sigma \sigma^{\prime \prime} x_{0} \frac{\partial}{\partial \theta}\left(\frac{\partial \phi}{\partial y_{0}}\right) \\
& -\tau x_{0}\left(\tau \frac{\partial \phi}{\partial x_{0}}\right)^{\prime}-\tau \tau^{\prime \prime} y_{0} \frac{\partial}{\partial \theta}\left(\frac{\partial \phi}{\partial x_{0}}\right) .
\end{aligned}
$$

After rearranglng,

$$
\begin{aligned}
\delta \mathrm{HO}_{0}= & \sigma \sigma^{\prime \prime} \frac{\partial}{\partial \theta}\left(x_{0} \frac{\partial \phi}{\partial y_{0}}\right)-\tau^{\prime \prime} \frac{\partial}{\partial \theta}\left(y_{0} \frac{\partial \phi}{\partial x_{0}}\right) \\
& -y_{0}\left(\sigma^{2} \frac{\partial}{\partial y_{0}} \phi^{\prime}\right)^{\prime}-x_{0}\left(\tau^{2} \frac{\partial}{\partial x_{0}} \phi^{\prime}\right)^{\prime},
\end{aligned}
$$

or, in polar coordinates,

$$
\begin{aligned}
\delta 10_{2}= & \frac{\sigma \theta^{\prime \prime}+\tau T^{\prime \prime}}{2} \frac{\partial^{2} \phi}{\partial \theta^{2}}+\frac{\sigma \sigma^{\prime \prime}-\tau^{\prime \prime}}{2} \\
& \times \frac{\partial}{\partial \theta}\left(r \sin 2 \theta \frac{\partial}{\partial r}+\cos 2 \theta \frac{\partial}{\partial \theta}\right) \phi-+\frac{\partial}{\partial r}\left(\frac{\theta^{2}+\tau^{\prime}}{2} \phi^{\prime}\right)^{\prime} \\
& +\left(r \cos 2 \theta \frac{\partial}{\partial r}-\sin 2 \theta \frac{\partial}{\partial \theta}\right)\left(\frac{\sigma^{2}-\tau^{2}}{2} \phi^{\prime}\right)^{\prime} .
\end{aligned}
$$

FInally, making use of symmetry, we obtain the defining equation for $\phi_{0}$

$$
n \frac{\partial^{2} \phi_{\mathrm{a}}}{\partial \theta^{2}}=-\Sigma,
$$

where

$$
\begin{aligned}
& \Sigma=\int_{-L}^{L} \frac{d z}{B} \frac{\partial p}{\partial \phi^{\alpha}}\left\{\left(\sigma \sigma^{*}+T T^{*}\right) \int_{0}^{\alpha} d z^{2} \cdot \frac{\partial^{2} \partial^{\prime}}{\partial \theta^{2}}\right. \\
& +\left(0 \alpha^{-}-2 \tau^{A}, \int_{0}^{\prime} d z_{j} \frac{\partial y}{\partial \theta}\left(r \sin z \theta \frac{\partial}{\partial r}+\cos 2 \theta \frac{\partial}{\partial \theta}\right) \phi^{\prime}\right. \\
& -r \frac{\partial}{\partial r}\left[\left(\sigma^{2}+r^{2}\right) b^{2}\right]^{*} \\
& \left.+\left(r \cos 2 \theta \frac{\partial}{\partial r}-\sin 2 \theta \frac{\partial}{\partial \theta}\right)\left\{\left(\sigma^{2}-T^{2}\right) \theta^{\prime}\right)^{\prime}\right\},
\end{aligned}
$$

and

$$
\pi=\int_{-c}^{2} \frac{d z}{B} \frac{\partial p}{\partial \phi}\left(\sigma \sigma^{\prime}+\tau \tau^{\prime \prime}\right) .
$$

This gaine integral, $\Pi$, appears in the familiar low - $\beta$ flute -instaulity criterion: $\Pi>0$.

At this stage several points need to be made. For one, we see that the perturbation procedure breaks down when the flute-instability parameter, $\Pi$, is actually equal to zero. Thus, thr re is apparently a b1furcation in the equilibrlum at ti.e marginal point. A second point is that the parallel cu-rent constraint is trivlally satisfied beyond the lateral boundary ot the plasma since the pressure vanishes. Consequently, there is not a unique value of $\phi_{0}$ in the vacuum region, any one wilt do. Different $\phi_{0}$ 's only correspond to different positions is the vacuum. With a conducting shell, $\phi_{0}$ is chosen to force $\psi=\psi^{*}$ on the boundary. In general, our only interest is in the flux-line geometry within the confined plasma; consequently, we do not examine the exterior region.

Thus, we see that Eqs. (45) and (52), atong with the appropriate definitions, completely determtne the equilibrium. All that remaing is to solve the elliptic differential equation (45), which ls done in the next section. From this point on, we consider anly the case in whlch the boundary is at Intinity, where $\phi^{\prime}$ must be well-behaved.

\section{SOLUTION FOR}

We solve Eq. (45) by translorming it to Poisson's equation in the stretched coordinates $\left(\tau^{-1} x_{0}, \sigma^{-1} y_{0}\right)$, which we then invert by means of the appropriate Green's function. In terms of the coordinates $(r, \theta)$ deflned in (31) and (32), the Green's function is

$\left.G(r, \theta) r^{\prime}, \theta^{\prime}\right)=\frac{1}{\pi} \ln \Lambda^{\prime}$

$\Lambda=\frac{1}{\tau^{2}}\left(r \cos \theta-r^{\prime} \cos \theta^{\prime}\right)^{2}+\frac{1}{\sigma^{2}}\left(r \sin \theta-r^{\prime} \sin \theta^{\prime}\right)^{\prime}$,

and $\Phi^{\prime}$ is

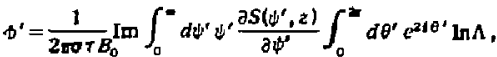

where we have again used (31) to express $r^{\prime}$ in terms of $\psi^{\prime}$.

Next, we integrate by parts with respect to $\psi^{\prime}$ to ob-

Pearlstein, Kaiser, and Newcomb 
tain

$$
\begin{aligned}
\phi^{\prime}= & \frac{1}{2 \pi 0+B_{0}} \operatorname{Im} \int_{0}^{-} d \psi^{\prime} S\left(\psi^{\prime}, z\right) \int_{0}^{2 z} d \theta^{\prime} e^{1 \theta^{\prime}} \\
& \times\left\{\ln \Lambda+\frac{1}{\Lambda}\left[r \cdot\left(\frac{\cos ^{2} \theta^{\prime}}{\tau^{2}}+\frac{\sin ^{2} \theta^{\prime}}{\sigma^{2}}\right)\right.\right. \\
& \left.\left.-r r^{\prime}\left(\frac{\cos \theta \cos \theta^{\prime}}{\tau^{2}}+\frac{\sin \theta \sin \theta^{\prime}}{\sigma^{2}}\right)\right]\right\} .
\end{aligned}
$$

We then transtorm the logarithmic term by integrating by parts on $\theta^{*}$ and obtain, alter some algebraic reduction,

$$
\begin{aligned}
s^{\prime}= & \frac{1}{2 \pi \sigma \pi B_{0}} \operatorname{Im} \int_{0}^{*} d t^{\prime} S\left(t^{\prime}, z\right) \int_{0}^{2 \pi} d \theta^{\prime} e^{\prime \theta^{\prime}} \frac{r^{\prime 2}}{\Lambda} \\
& \times\left[\frac{\cos \theta^{\prime}}{\tau^{2}}+i \frac{\sin \theta^{\prime}}{\sigma^{2}}-\frac{r}{r}\left(\frac{\cos \theta}{T^{2}}+i \frac{\sin \theta}{\sigma^{3}}\right)\right] .
\end{aligned}
$$

We evaluate the integral over $\theta^{\prime}$ by converting it io a contour integral over the unit circle: $e^{\prime \theta^{\prime}}=6$,

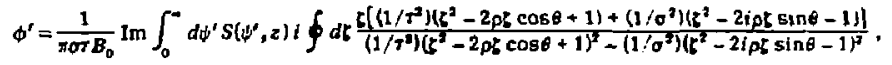

where $\rho$ a $r / r^{\prime}$. The $\xi$ integrand has lour poles. Two are roots of

$$
r^{2}-2 p x^{-} r-\mu^{-}=0,
$$

and $t$ wo are roots of

$$
t^{2}-2 p \times t-\mu^{*}=0
$$

where

$$
x^{*}=\frac{1}{2}\left(e^{10}-\mu^{*} e^{-t *}\right) \text {, }
$$

and

$$
\mu^{4} \equiv(\tau \neq \sigma) /(\gamma \neq \sigma) .
$$

We henceforth assume that $r>0$, Implying that $\mu^{*}>1$, $\mu^{*}<1$. The final answer will be independent of this cholce. We write the roots as

$$
\xi ;=p x^{*}+\Gamma_{j}^{*}, j=1,2,
$$

where

$$
r_{j}^{\prime}=F\left(-1 V\left(\rho^{2}\left(x^{4}\right)^{2}+\mu^{4}\right)^{1 / 2}, j=1,2 .\right.
$$

It is then possible to show that the residue at each of the poles is given by the general formula

$$
R_{j}=(i / 4) \mu^{*}\left(0 x^{*} / \Gamma_{j}^{*}+1\right) \text {. }
$$

With Eq. (61), Eq. (56) reduces to

$$
\phi^{\prime}=-\frac{1}{2 \sigma} \frac{1}{B_{0}} \operatorname{Im} \int_{0}^{-} d \psi^{\prime} S\left(\psi^{*}, z\right) \sum_{\text {Nos }} \rho \frac{\mu^{*} x^{*}}{\Gamma_{j}^{*}},
$$

the sum including only those poles lying inside the unit circle.

All that remains is to locate the poles. Consider those at $\zeta=c$; . We note that at $\rho=1,\left|\xi_{j}\right|$ is independent of 8. In particular,

$$
\left.\left|\zeta_{1}\right|\right|_{. .1}=1,\left.\left|\xi_{2}^{*}\right|\right|_{\text {,.t }}=\mu^{*} .
$$

We also observe that

$$
\left.\left|t_{i}\right|\right|_{p=0}=\sqrt{\mu^{*}},
$$

independent of $\theta$. Now consider $\mid \xi j$ ! for $\theta=0$. We see that

$$
\left|\zeta_{j} \|_{\theta=a}=-(-1)^{\frac{1}{2} \rho} \rho\left(1-\mu^{*}\right)+\right| \rho^{2}\left(l-\mu^{*}\right)^{2}+\left.\mu^{*}\right|^{1 / 2} \text {, }
$$

a $n$. notonic function of $p$. For $\theta=\pi / 2$, there are $t$ wo possi: ilities, depending on the value of $\rho$. If $\rho<2 \sqrt{\mu^{*}}$ $\left(1+\mu^{+}\right), \Gamma ;$ is real, and

$$
\left.\left|z_{i}\right|\right|_{\text {ear } 1}=\sqrt{\mu^{*}} \text {. }
$$

If $p>2 \sqrt{\mu^{*}} /\left(1+\mu^{*}\right)$, on the other hand, $r ;$ is purely imaginary, and we find

$$
|6 ;|_{0 \times 5 / 2}=1 \rho\left(1+\mu^{*}\right)+\left.(-1)^{\prime}\right|^{1} \rho^{2}\left(1+\mu^{*}\right)^{2}-\left.\mu^{*}\right|^{1 / 2} \text {, }
$$

again, a monotonic lunction of $\rho$. Finatly, it can be shown that $d\left|c_{j}\right| / d \theta=01$, and only if, $\theta=0$ or $\mathrm{A} / 2$, or if $\rho=0$ or 1 . The net result of the foregoing considerations is that all possible values of $|\xi ;|$ are confined to the shaded region of Fig. 1.

A similar diagram could be constructed for $|\zeta ;|$ by recognizing that

$$
(\xi ;) *=-\mu t_{i}^{*} \text {. }
$$

or

$$
\left|\zeta_{i}^{-1}=\left(1 / \mu^{*}\right)\right| \boldsymbol{c}_{;}^{*} \mid \text {. }
$$

From Fig. $J$ and Eq. (63) we conclude that for $p<3$, i.e., $">d$, the contributing poles are $\xi_{;}$. Referring to Eq. (62), we see that in this case the sun over the poles is proportional to $\left(\Gamma_{i}^{-1-1}+\left(\Gamma_{z}^{-}\right)^{-1}=0\right.$. The effect is to restrict the $\psi^{\prime}$ integration to the range $\left[0, w_{1}\right.$. Agaln referring to Fig. 1 and Eq. (63), we see that, for $\rho>1\left(\psi^{r}<\psi\right)$, only the poles at $\xi=\xi_{1}^{+}$contribute.

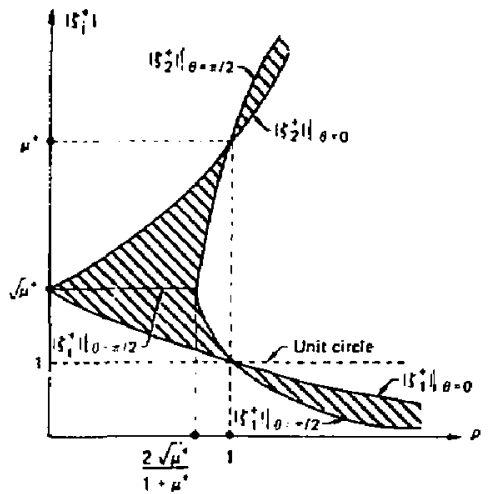

FIG. 1. Sketch showing the locatton of the poles $r=r$; relative to the boundary of the unit circle $|5|=1$. 
Thus, from Eq. (62),

$$
\phi^{\prime}=-\frac{1}{2 \sigma \tau} \frac{1}{B_{0}} \operatorname{lm} \int_{0}^{\prime} d \psi^{\prime} S\left(\psi^{\prime}, z\right) \rho\left(\frac{\left.\mu^{+} \mathbf{Y}^{*}+\frac{\mu^{*} \chi^{-}}{\Gamma_{1}^{*}}\right)}{\Gamma_{1}^{-}}\right) .
$$

We can simplify further by noting that $\left(x^{*} / \Gamma_{i}^{*}\right)^{*}=x^{*} / \Gamma_{i}^{*}$. Therefore, finally,

$$
\begin{aligned}
\Phi^{\prime}= & -\frac{2}{(\tau+\sigma)^{2} B_{0}} \frac{1}{\mu} \operatorname{Im} \int_{0}^{\phi} d \psi^{\prime} S\left(\nu^{\prime}, z\right) \\
& \times\left(1+4 \frac{\psi}{\psi} \frac{\mu e^{(2)}}{\left(1-\mu e^{(2 \sigma)^{2}}\right.}\right)^{-1 / 2} .
\end{aligned}
$$

In Eq. (64) and henceforth we deflne

$$
\mu=\mu^{\circ} \text {. }
$$

After considarable algebra this solution has been shown to satisfy Eq. (45) by direct substitution, It can eastly be shown that if $S$ is a polynomial in $\downarrow$ of order $N$, then for $\psi<\psi_{a}, \phi^{\prime}$ has harmonice up to $\sin 2(N+1) \theta$. On the other hand, for $\psi>\psi_{g}$, all harmonics are generated (although, of course, falling off $\left.28 \psi^{* *}\right)$. To complete the solution, the stream furction itself is given by

$$
\phi=\int_{0}^{\infty} \phi^{\prime}\left(\psi, \theta, z^{\prime}\right) d z^{\prime}+\phi_{0}(\psi, \theta),
$$

where $\phi_{0}(\psi, \theta)$ is obtained from Eqs. (52) through (54).

In Appendix B we write down the explicit equations for the equilibrium inside the plasma source for the following pressure profiles:

$$
p_{u}=\left(1-\psi / \phi_{B}\right) \hat{p}_{n}(B),
$$

and

$$
\hat{p}_{11}=\left(1-\psi / \psi_{\Delta}\right) \hat{p}_{11}(B),
$$

where $\hat{D}_{11}(B)$ is chosen to flt the various tandem mirror configurations, and the $\psi$ dependence is assumed to be the same in different reglons of the system. Although configurations in which the weighting function taries from one region to another are conceivable, we do not consider such complications.

\section{v. DIscussion}

It should be emphasized that equilibrium based on the anisotropic magnetohydrodynamic equations does not in itself guarantee long time equ!librium. These equa tions only assume $(\mu, n)$ Invariance. To ensure equiLibrium on a drift time scale, the particle distribution must satisly the drift kinetic equation

$$
\frac{\partial F}{\partial t}+\frac{c}{e}\left(\frac{\partial K}{\partial \psi} \frac{\partial F}{\partial \theta}-\frac{\partial K}{\partial \theta} \frac{\partial F}{\partial \psi}\right)=0,
$$

where the partial derivatives imply $(\mu, \Omega)$ held constant; $\boldsymbol{K}$ is the Hamiltonian defined in terms of the action as

$$
J=\int d s[2(K-\mu B-e \phi) / m]^{1 / 2} ;
$$

and its dertvatives are proportional to the respective $\nabla B$ drifts. Now, the $\nabla B$ drifts due to finite $\beta$ are large (of order $\lambda^{-2}$ ) compared with the curvature drifts and since $B$ is a futction of $\psi$ only ( $\theta$ dependence is of order $\lambda^{2}$ ), the drift kinetic equation is automatically satisfied to the lcwest order in $\lambda$ since $F$ is not a function of $\theta$.
Next, before we present a detailed equilibrium, the following points shoutd be made. We note that the $\beta$ correction to the flux surface defined by $p(\psi, B)$, is in versely proportional to the instability criterion f note the definition of $\Pi$, Eq. (54)]. At the marginal point the perturbation expansion breaks down, i.e., $\phi_{0} \rightarrow \infty$, which implies a bifurcation in the equilitrium. Whether the other equilibrium is physical or not we cannot say al this time.

Another observation regrarding this term is that the signs of $\phi_{0}$ and $n$ are the same. The numerator on the right side of Eq. (52) for the tandem mirror configuration is pos!tive deilnite; to see this consider Eq. (B9). Glven that the central cell is generally axisymmetric over most of its length, only the second term proportonal to $k_{1}$ in Eq. (B7) is scaled with this longest length. Alt other terms in the Intesrand are zero in the axisymnetrle region and can be dropped. Then, for this term we have

$$
\begin{aligned}
\Sigma= & \frac{16 \psi^{2}}{3 B_{0} \psi_{B}} \sin 4 \theta \int_{-L}^{L} d z \frac{\hat{p}}{B}\left(\sigma \sigma^{\prime \prime}-\tau \tau^{\prime \prime}\right) \\
& \times \int_{0}^{\prime} \frac{d z^{\prime}}{B(\sigma+\tau)^{2}} \int_{\alpha^{\prime}}^{1} d z^{\prime \prime} \frac{\hat{\rho}}{B}\left(\sigma \sigma^{\prime \prime}-\tau \tau^{\prime \prime}\right) .
\end{aligned}
$$

Now define $z= \pm l$ as the boundary of the axisymmetric region such that $l /(L-I) \gg 1$ and consequently,

$$
\begin{aligned}
\Sigma= & \frac{32 \psi^{2}}{3 B_{0} \psi_{\theta}} \sin 4 \theta \int_{t}^{L} d z \frac{\hat{p}}{B}\left(\sigma \sigma^{\prime}-\tau \tau^{\prime \prime}\right) \\
& \times \int_{0}^{1} \frac{d z}{B(\sigma+\tau)^{2}} \int_{1}^{L} d z \frac{\dot{p}}{B}\left(\sigma \sigma^{\prime \prime}-\tau \tau^{\prime \prime}\right) \\
\approx & \frac{\theta}{3} \frac{\psi^{2}}{B_{0} \psi_{\theta}} \sin 4 \theta\left(\int_{1}^{2} d z \frac{\dot{p}}{B}\left(\sigma \sigma^{\prime \prime}-\tau \tau^{\prime \prime}\right)\right)^{2} \int_{0}^{1} \frac{d z}{B},
\end{aligned}
$$

and finally we have

$$
\omega_{0}=-\frac{1}{12} \frac{\psi^{2}}{B_{0} \psi_{B}} \int_{0}^{1} \frac{d z}{B} \frac{\left|\int_{l}^{L} d z \bar{p}\left(\sigma \sigma^{\prime \prime}-\tau \tau^{\prime \prime}\right) / B\right|^{2}}{\int_{0}^{L} d z \hat{p}(\sigma o} \sin 4 \theta
$$

The form of Eq. (71) is the same as that derived by Stupakov ${ }^{3}$ who considered only the case where $l$ was very large.

Now the flux surface at the midplane is easily seen to be, Eqs. (39) and (40),

$$
\frac{1}{2}\left[r(0)^{2} B(0)\right]=\psi(1+2 k)+B_{0} \frac{\partial \phi_{0}}{\partial \theta} .
$$

Hence if $\Pi<0$ (denominator $>0$ ), which it must be for a stable configuration, the octupole distortion squares off the surface. With the other sign the surface exhibits a diamond-shaped distortion.

This property explains the difference between our results and those of Stupakov ${ }^{3}$ Ihat led lo a diamondshaped distortion. He argued that a proper boundary condition was $j_{n}=0$ at the mirror peak separating the central cell trom the plug, thus discarding any influ ence of the plug on the central cell. In general, the flute-instability criterion [ $\mathrm{Eq} .(54)]$ is positive (unstable) for the central-cell alone. Because of this change in sign, he was led to the constraint that the central cell could not be too long or else field lines would migrate to the walls because of the finlte distor- 

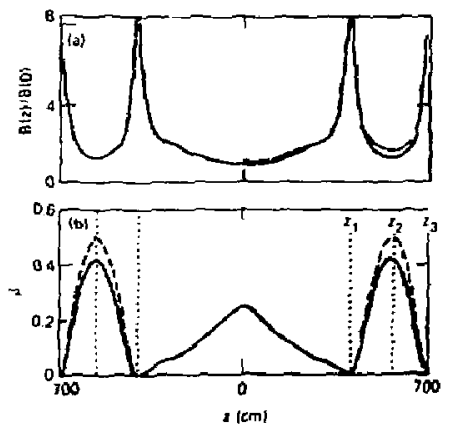

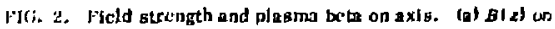
axjs, both in tocue (dashed curve) and with plasme present (kolld curve), normafized to $\left(B_{0} \mathrm{f}\right.$. (b) Plasma beta on axds. $\beta_{1} \equiv 2_{1} / B_{y}^{2}$ is shown dashed and $\left.\beta \equiv \phi_{1}+p_{11}\right) / B_{p}^{2}$ solld. Vertical dorted lines murk the inborrd mirrar peaks and midD'ane $s$ of the end cells,

tlon of the flux. As we will subsequently show we obtain the opposite eftect, that is, because beta is Inite, field lines reduce their maximum excursion from the axis in the central-cell region.

Now, which boundary candition is correct? Obviously, if we assume thal the mirror is isolated from the outside world, $j_{1}=0$ at the axial edge of the confined plasma. This boundary condition, along with the lateral boundary condition, completely determipes the equilibrium. There is no freedom to specify the parad lel current at the intertace of the central cell and the plug. We, of course, find that it is not simultaneously zero at both intertaces.

We naw present results based on the thear flux dependence of the pressure prolile for a standard tandem
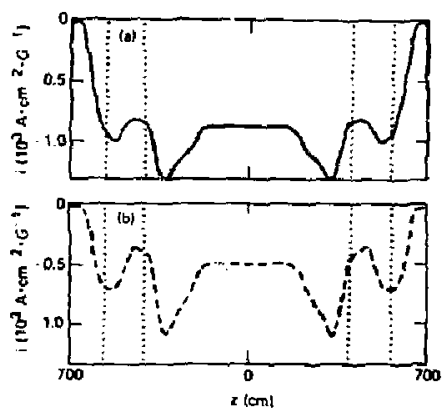

FIG. 3. parallel curreaf per unit magoets dux calculated from Iq. (22) on the fleld Itne $\phi=\psi, \theta=\pi / 4$. In ( $k$ ) the equilibrium inagnete fyeld use used in the calculatton, whlle in (b) the vacuum field aas used. Vertlcal dotted lines are as in Fig. 2.

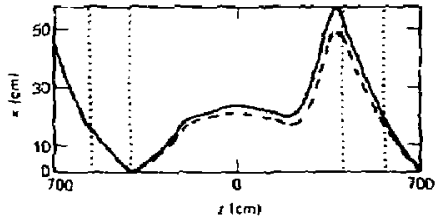

Ftc. 4. The $*$ coordinate of the fleld Iine $4=I_{B}, \theta=x / A$, in encuo (dashed curvel and with plasma present isolid curve). vertical dotted lines are as in Fif. 2.

mirror contiguration (the TMX Upgrade) for which $\left(b_{\mathrm{s}}\right)_{\mathrm{v}}$ $=3 \times 10^{2} \mathrm{G}$, ant $\psi_{\mathrm{g}}=1.35 \times 10^{\circ} \mathrm{G}^{\mathrm{c}} \mathrm{cm}^{3}$. The computer code $E F F^{\prime}$ and the experimental coil configuration werc used to generate the vacuum field functions $B(z)$, oxl an a Sime grte (typtcaliy 200-400 polnts). Cuble spline interpolation was used between grid points \$0 that the quadrztures arising in the calculation of $p$ could be evaluated usiog the Gear method. ${ }^{\text {* }}$ In Fig. 2 we show the dependence of $\bmod -B$ and pressure on $z$. In FIg. 3, we plot the parallel current per unit flux as a function of $z$ in both the total fiteld and the vacuum field. In Fig. 4, we compare a lield-line trajectory at finite bela with its trajectory in the vacuum for the same flux coordinates. In Figs. 5 and 6 , we have simllar comparisons for the normal and geodesic curvatures. Nole that although (he $O\left(\lambda^{2}\right)$ quantity 24 a approaches unity in the transition regions for the extreme case shown $\left(4=f_{a}\right)$, it is small over the bulk of the plasma because it scales as $x^{2}$. In Fig. T, we compare flux surfaces in the finite-beta field with thase in the vacuum field at the midplane of the machine, the mir ror peaks, and the midplane of the plug. We see squaring off of the Rux surface at the midplane of the machine coctupole distortion). We also note the reduced eccentricity at the inboard mirror, the plasma currents are attempting to recircularize. Conversely, ite eccentricity at the outbogrd malror is increased.
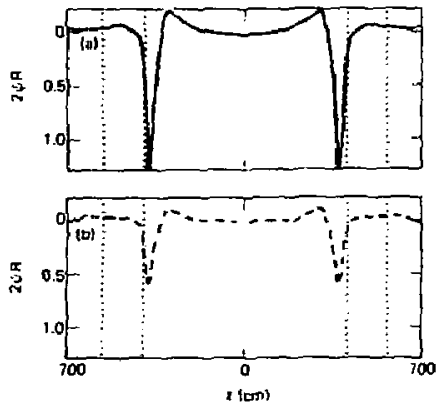

FiG, 5. The quintity 269 on the sleld ine $t=t_{g}, \theta=7 / 4$, to both the equltibrium magnetac fleld (a) and in urcuo (b). Vertieal dated lises are in Fig, 2 .

Pearlstein, Kaiger, and Newcomb 

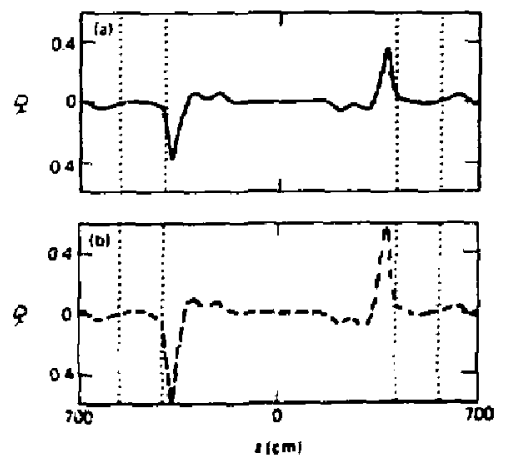

FIG. 6. The peodeste curiature on the ficld line $t=0$ th, $4 \pi / 4$, If both the ejullibrium ing gnetle field $(a)$ and in torwo (b). vertlcal dotted IInes are as in Fig. 2.

In these comparisons we have used

$$
\hat{\beta}_{\perp}=\bar{p}=\left(\beta_{c e r} / 2\right) B^{2}(0), \quad z<z_{1}
$$

and

$$
\hat{p}_{2}=\frac{\beta_{21}=B^{z}\left(z_{2}\right)}{2} \frac{B_{u}^{2}-B^{2}}{B_{-1}^{3}-B^{2}\left(z_{2}\right)}+5 p_{1}, \quad z_{1}<z<z_{3},
$$

where

$$
\begin{gathered}
s p_{1}=\frac{\beta_{\alpha x}}{2} B^{z}(0) \frac{B^{3}(z)-B^{2}\left(z_{1}\right)}{B^{3}\left(z_{1}\right)-B^{2}\left(z_{2}\right)}, z_{1}<z<z_{2}, \\
B p_{1}=6 \beta_{c e} \frac{B^{2}(0) B^{2}(z)\left[B(z)-B\left(z_{2}\right)\right]\left[B_{m}-B(z)\right]}{\left[B\left(z_{1}\right)+B\left(z_{2}\right)\right]\left[B_{m}-B\left(z_{2}\right)\right]^{3}}, \\
z_{2}<z<z_{3}, \\
\text { where } B_{m}=\min \left[B\left(z_{1}\right), B\left(z_{3}\right)\right] .
\end{gathered}
$$

To close, $6 f_{n}$ is obtatred from the integral of the parallel pressure balance equation

$$
\frac{c}{d B} \frac{p_{\|}}{B}=-\frac{p_{1}}{B^{2}}
$$

Rote that these combinations were chosen to force $p_{\mathrm{v}}$ to ye zero at the plasma boundary. Aiso note that $z_{3}$ is the position of the inboard mirror, $z_{3}$ is the position of the outboard mirror, and $z_{2}$ is the position of the plug Ininimum (see Fig. 2). These choices are used to represent the penetration of the central-cell plasma and to identify the solenold and piug bet 2 values. For these computations $\beta_{c}=0.25$ and $\beta_{p}=0.5$. These high values - 7 re picked to emphasize the effects of the plasma currents. At lower values the results are gualitatirely the same.

We conclude with a word of caution. The analysis is perturbative and so fleld tine displacements should be small relative to their vacuum positions to be beliered. slso, to quantify the accuracy it is necessary to calculate to next order in $B$, a project not contemplatel at this time.
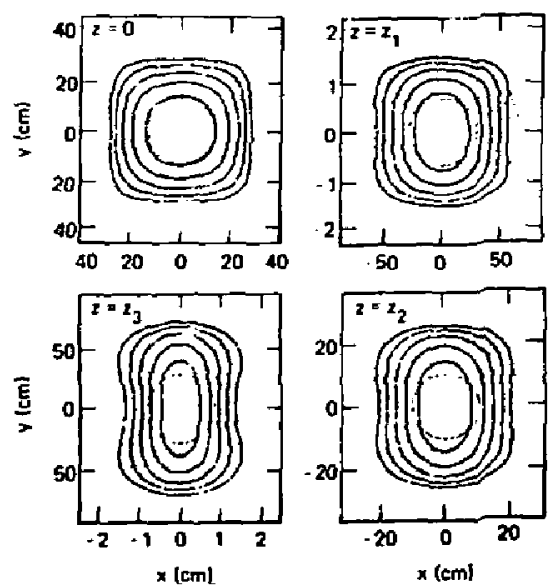

FIG. 7. Constant-pressure-surtace crose sectione perpendiching to the magnetic axls in vacuo (dotted curves) and at IInite beta (solld curves). Contours are at equally spaced fux tntervals shown at four different axdal pos!lions.

\section{ACKNOHLEDGMENTS}

We have benefited greatly trom the helpful comments of L. S. Jall.

This work was performed under the ausplces of the U. S. Department of Energy by the Lawrence Livermare National Laworatory under contract number W-7405ENG -48 .

\section{APPENDIX A. LONG-THIN DIFFERENTIPL GEOMETRY}

Here, we derive Eqs. (17) and (21), which, with Eq. (22), determine the field-line geometry. Both follow from the large-aspect-ratio limit of a standard dif ferenlial geometric relationship among the variables of any invertible coordinate transformation. This result is most concisely stated if we denote the Cartesian co-

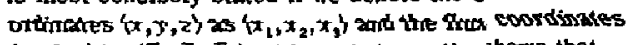
$(\alpha, \beta, s)$ by $\left(\bar{x}_{1}, \bar{x}_{2}, \bar{x}_{9}\right)$. Then, it is easily shown that

$$
\frac{\partial \bar{x}_{1}}{\partial x_{1}}=\epsilon_{i=n} \epsilon_{1+1} \frac{\partial\left(x_{k}, x_{1}\right)}{\partial\left(\bar{x}_{m}, \bar{x}_{n}\right)} \frac{\partial\left(\bar{x}_{1}, \bar{x}_{2}, \bar{x}_{3}\right)}{\partial\left(x_{1}, x_{2}, x_{3}\right)} \text {, }
$$

where $\epsilon_{i m n}=0$ if any two indices are equal and $= \pm 1$ according to whether $(i, m, n)$ is an even or odd permutation of $(1,2,3)$; the standerd notation for the Jacobian is used; and the summation convention is not used. An equivalent equation with barred and unbarjed variables interchanged clearly also holds.

From Eq. (10) we find that

$$
\frac{a\left(\bar{x}_{1}, \bar{x}_{2}, \bar{x}_{3}\right)}{\partial\left(x_{1}, x_{2}, x_{3}\right)}=\nabla \alpha \times \nabla \beta \cdot \nabla s=B \cdot \nabla s=B \text {. }
$$

Then, Eq. (17) follows immediately from the long-thin limit of the special case of Eq. (A1) for which $i=j=3$;

$$
\frac{\partial s}{\partial z}=\frac{\partial(x, y)}{\partial(\alpha, \beta)} B
$$


To $O(\lambda)$, however, Eq. (14) implies that

$$
\frac{\partial \bar{r}_{1}}{\partial r_{1}} \cdot \frac{\partial x_{3}}{\lambda \bar{r}_{1}}=\delta_{13},
$$

with which Eq. (A3) becomes

$$
\frac{\partial(r, y)}{\partial(\alpha, \beta)}=B^{2}
$$

i.e., Eq. (17).

To obtaln Eq. (21) we note that in the Large-zspectratio it nit, the components of the unit vector $b$ are [see Eqs. (15), (28)]

$$
b_{x}=x^{\prime}, b_{y}=y^{\prime \prime}, b_{d}=1 \text {. }
$$

Inserting Eqs. (A5) in Eq. (8) glves

$$
i=\hat{b} \cdot \nabla \times \hat{b}=-x^{\prime} y^{\prime \prime}+y^{\prime} x^{n}+\frac{\partial y^{\prime}}{\partial x}-\frac{\partial x^{\prime}}{\partial y^{\prime}} .
$$

The tirst twe terms on the right side of Eq. (AB) are $O\left(\lambda^{2}\right)$ relative to the last two, leaving

$i=\frac{\partial y^{\prime}}{\partial x}-\frac{\partial x^{\prime}}{\partial y}=\frac{\partial y^{\prime}}{\partial \alpha} \frac{\partial \alpha}{\partial x}+\frac{\partial y^{\prime \prime}}{\partial \beta} \frac{\partial \beta}{\partial x}-\frac{\partial x^{\prime}}{\partial \alpha} \frac{\partial \alpha}{\partial y^{\prime}}-\frac{\partial x^{\prime}}{\partial \beta} \frac{\partial \beta}{\partial y}$.

If we now use Eqs. (A4) in Eqs. (A1) to evaluate the necessary partial derivatives, we tind that Eq. (A7) becomes

$$
\begin{aligned}
i & =B\left(\frac{\partial y^{\prime}}{\partial \alpha} \frac{\partial y}{\partial \beta}-\frac{\partial y^{\prime}}{\partial \beta} \frac{\partial y}{\partial \alpha}+\frac{\partial x^{\prime}}{\partial \alpha} \frac{\partial x}{\partial \beta}-\frac{\partial x^{\prime}}{\partial \beta} \frac{\partial x}{\partial \alpha}\right) \\
& =B\left(\frac{\partial\left(x^{\prime}, x\right)}{\partial(\alpha, \beta)}+\frac{\partial\left(y^{\prime}, y\right)}{\partial(\alpha, \beta)}\right),
\end{aligned}
$$

which is $\mathrm{Eq}$. (21).

\section{APPENDIX B. LINEAR AND OUADRATIC PRESSURE PROFILES}

In this appendix, we obtain the explicit solutions for the two pressure profiles given by Eqs. (67) and (68). First, define

$$
k_{1}(z)=\frac{4}{B} \frac{1}{\left(0+T^{2}\right.} \int_{2}^{L} d z \frac{E}{B}\left(00^{*}-J T^{\prime \prime}\right),
$$

and

$$
k_{2}(z)=\mu\left(\hat{p}_{1} / B^{2}\right)^{\prime} .
$$

Now considering first the linear pressure profile. we have from Eqs. (42), (46), and (64)

$$
\begin{aligned}
\phi_{1}^{\prime}= & -\frac{1}{2 \mu B_{0}} \operatorname{Im} \int_{0}^{+} d \psi^{\prime}\left[\left(k_{1}+k_{2}\right)-\frac{\psi^{\prime}}{\psi_{2}}\left(k_{1}+\frac{k_{2}}{2}\right)\right] \\
& \times\left(1+\frac{4 \psi^{\prime}}{\psi} \frac{\eta}{(1-\eta)^{2}}\right)^{-1 / 2},
\end{aligned}
$$

with the definition

$$
\eta=\mu e^{2 i \theta} \text {. }
$$

Integrating by parts twice generates

$$
\begin{aligned}
\phi_{L}^{\prime}= & -\frac{\psi}{2 \mu B_{0}} \operatorname{Im}\left[\left(k_{1}+k_{2}\right) \frac{1-\eta}{2 \eta}(1+\eta-1+\eta)\right. \\
& -\frac{\psi}{\psi_{a}}\left(k_{1}+\frac{k_{2}}{2}\right) \frac{1-\pi^{2}}{2 \eta}-\frac{1-\eta}{12 \eta^{2}}\left[(1+\eta)^{9}-\left(1-\eta_{i}^{131}\right],\right.
\end{aligned}
$$

or collecting ternis,

$$
\begin{aligned}
\phi_{1}^{\prime} & -\frac{\psi}{2 \mu B_{a 1}} \operatorname{Im}\left[\left(k_{2}+k_{2}\right)(1-\eta)\right. \\
& \left.-\frac{\vdots}{\psi_{2}}\left(k_{1}+\frac{k_{2}}{2}\right)\left(\frac{1}{2}-\frac{2}{3} \eta+\frac{1}{6} \eta^{2}\right)\right] .
\end{aligned}
$$

Taking the imaginary part produces the answe $r$

$$
\begin{aligned}
s_{1}^{\prime}= & \frac{\ddagger}{B_{0}}\left\{\left[\frac{1}{2}\left(k_{1}+k_{2}\right)-\frac{j}{3 \dot{i}_{\Delta}}\left(k_{1}+\frac{k_{2}}{2}\right)\right] \sin 2 \theta\right. \\
& \left.+\mu\left(k_{1}+\frac{k_{1}}{2}\right) \frac{j}{12 \psi_{a}} \sin 4 \theta\right\}
\end{aligned}
$$

Turning to the expresstons for $s_{0 .}$ Eqs. (52) through (54), and noting that $k_{1}$ and $k_{2}$ are even functions of 2 , we have for the numerator:

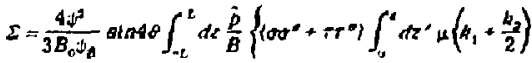

$$
\begin{aligned}
& +\left(\sigma \sigma^{n}-\tau \tau^{n}\right) \int_{a}^{n} d z^{\prime}\left(k_{1}+\frac{k_{2}}{2}\right)+\frac{1}{4}\left[\left(\sigma^{2}+\tau^{2}\right) \mu\left(h_{1}+\frac{h_{2}}{2}\right)\right. \\
& \left.\left.+\left(\sigma^{2}-\tau^{2}\right)\left(k_{1}+\frac{k_{2}}{2}\right)\right]^{4}\right\} \text {. }
\end{aligned}
$$

Note that the term linear in $\downarrow$ cancels. Next integrat ing parts and combining the integrated part, we have with

$$
\left(\frac{\hat{p}}{B}\right)^{\prime}=\frac{B}{2}\left(\frac{\hat{p}_{t}}{B^{2}}\right)^{\prime}
$$

[see Eq. (2)] and Eq. (25),

$$
\begin{aligned}
& D_{0,1}=-\frac{\psi^{2}}{2 B_{0} \psi_{B}}\left\{\int _ { - L } ^ { c } d z \frac { \hat { p } } { B } \left[\left(\sigma 0^{\prime \prime}+\tau \tau^{\prime \prime}\right) \int_{0}^{c} d \tau^{\prime} \mu\left(h_{1}+\frac{k_{7}}{2}\right)\right.\right. \\
& \left.\left.+\left(\sigma \sigma^{n}-\tau \tau^{n}\right) \int_{0}^{c} d z^{\prime}\left(k_{1}+\frac{k_{2}}{2}\right)\right]+\frac{B_{0}}{4} \int_{-2}^{L} d z k_{2}\left(k_{1}+\frac{k_{2}}{2}\right)\right\} \\
& \times \frac{\sin 4 \theta}{\int_{-L}^{L} d z \dot{p}\left(\sigma \sigma^{*}+\tau \tau^{\prime}\right) / B} .
\end{aligned}
$$

Next, we turn to the quadratic smusce. Here we have

$$
\begin{aligned}
\phi_{2}^{\prime}= & -\frac{1}{2 \mu B_{0}} \text { Im } \int_{0}^{0} d \psi^{\prime}\left\{\left(k_{1}+k_{2}\right)-2 \frac{\psi^{\prime}}{\psi_{B}}\left(k_{1}+\frac{k_{2}}{2}\right)\right. \\
& \left.+\left(\frac{\psi^{\prime}}{\psi_{\Delta}}\right)^{2}\left(k_{1}+\frac{k_{2}}{3}\right)\right\}\left(1+\frac{4 \psi^{\prime}}{\psi} \frac{\eta}{(1-\pi)^{2}}\right)^{-1 / 2} .
\end{aligned}
$$

Integrating by parts three times protuces

$$
\begin{aligned}
\sigma_{2}^{\prime}= & -\frac{\psi}{2 \mu \bar{B}_{0}} \operatorname{Im}\left(\left(k_{1}+k_{2}\right)(1-\eta)-\frac{\psi}{\psi_{a}}\left(k_{1}+\frac{k_{2}}{2}\right)\right. \\
& \times\left(1-\frac{4}{3} \eta+\frac{1}{3} \eta^{2}\right)+\left(\frac{\psi}{\psi_{a}}\right)^{2}\left(k_{1}+\frac{k_{z}}{3}\right)\left\{\frac{1-\eta^{2}}{2 \pi}-\frac{1-\eta^{2}}{6 \eta^{2}}(1+\eta)^{2}\right. \\
& \left.\left.+\frac{1}{60^{3}}(1-\eta]\left[(1+\eta)^{3}-(1-\eta)^{*}\right\}\right\}\right),
\end{aligned}
$$

Fhere we have used Eq. (B5) (note tlat the term quad. ratic in $\psi / \psi_{a}$ is multiplied by two here). Again collecting terms in the braces we obtain for that term

$$
\left(\frac{\psi}{\psi_{B}}\right)^{2}\left(k_{1}+\frac{k_{n}}{3}\right)\left(\frac{1}{3}-\frac{\eta}{2}+\frac{\eta^{2}}{5}-\frac{\eta^{3}}{30}\right) \text {. }
$$


Finally we have for the imaginary part

$$
\begin{aligned}
\Phi_{2}^{\prime}= & \frac{\psi}{B_{0}}\left[\frac{1}{2}\left(k_{1}+k_{2}\right) \sin 2 \theta-\frac{1}{2} \frac{\psi}{\psi_{\theta}}\left(k_{1}+\frac{k_{1}}{2}\right)\right. \\
& \times\left(\frac{4}{3} \sin 2 \theta-\frac{1}{3} \mu \sin 4 \theta\right)+\frac{1}{2}\left(\frac{\psi}{\psi_{B}}\right)^{2}\left(k_{1}+\frac{k_{2}}{3}\right) \\
& \left.\times\left(\frac{1}{2} \sin 2 \theta-\frac{1}{5} \mu \sin 4 \theta+\frac{1}{30} \mu^{2} \sin 6 \theta\right)\right] .
\end{aligned}
$$

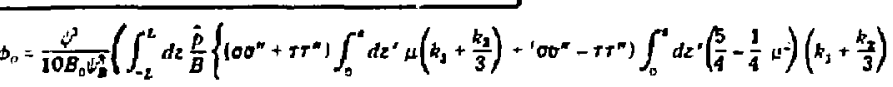$$
\left.\left.-\left[\frac{\mu}{2} \sigma\left(k_{1}+\frac{k_{2}}{3}\right)\right]\right\}\right) \frac{\sin 4 \theta}{\int_{-2}^{2} d z \hat{p}\left(\sigma \sigma^{e}+r^{\alpha}\right) / \theta}
$$

Closing, we compute the expression for $\phi_{n-}$ We immediately see that

$$
\phi_{0.2}=2 \omega_{0,1}+\delta \phi_{0}
$$

where $\delta g_{0}$ comes from the new tern proporional to $\left(k_{1}+k_{2} / 3\right)$ in Eq. (Bj2). For that term we have

and, atter integrating by parts

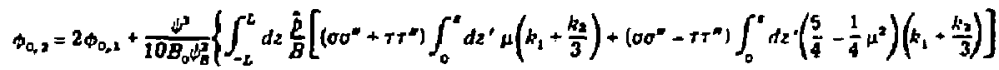

$$
\begin{aligned}
& \left.+\frac{B_{0}}{4} \int_{-z}^{\mathrm{L}} d z^{\prime} k_{1}\left(k_{1}+\frac{k_{2}}{3}\right)\right\} \frac{\sin 4 \theta}{\int_{-t}^{L} d z \dot{\rho}\left(0 \sigma^{*}+5 T^{\prime}\right) / \theta} \text {. }
\end{aligned}
$$

'L. S. Hall and B. MeNamare, Fhys, Flulds 18, 552 (1975). ${ }^{5} B$. MeNamara, D. V. Anderson, J. K. Boyd, J. A. Byers, R. Cohen, T. A. Cutler, L. S. Hall, R. F. Posk and M. E. Renoink, in Plasma Physies and Controlled Nuclear Fusion Resedreh international Atomle Esetgy A gency. Vteans, 19771, Yol. 1, D. 161.

3G. V. Stuptkov, Fiz. Platiny 5, 871 (1979) (Sov. J. Plasma Phy5. 5, 486 (1979)].

W. A. Newcomb, Lawrence Luvermore Natlonal Leboratory
Report, CCRL-85188 (1980).

$s_{H}$. Grad, in Praccedings of Symposia in Applifed thathemafics Umerican Mathematical Soclety. Prouddence. Rhode Island, 1967), Vol. XVIT, p. 162.

${ }_{6}$. P. Fureth and M. x. Hosenblunth, Phys. Flutds 7, 764 (1964).

'S. J. Sacketr, Latirence Livermore National Laboratory Report, UCAL-52402 (1978).

'C. W, Gear, Commud, ACM 14, 176 (1971). 



$$
\text { is: }
$$




\section{APPENDIX B}

GYROKINETIC MAGNETOHYDRODYNAMICS (MHD)

W. M. Nevins and T. E. Kaiser

\section{INTRODUCTION}

Quadrupole tandem mirrors rely for MHD stability on high pressureweighting of regions of favorable curvature (MHD anchors) relative to those of destabilizing curvature. This guarantees stability to curvature-driven modes when the plasma $\beta$-values in the unstable region of destabilizing curvature is less than some critical value that depends on the value of $\beta$ in the anchors and on details of the magnetic geometry.

To determine the critical $\beta$-value, we must formulate and solve the equation of motion for the modes of interest. At low $B$, the characteristic perturbations are electrostatic flute modes whose stability criterion is simple and well-known. ${ }^{B-1,9}$ At higher values of $\beta$, magnetic-field perturbations make $f$ lux-line bending possible. This allows modes to localize in regions of unfavorable curvature. The stability properties of such ballooning modes ultimately will determine the critical $\beta$-values achievable in tandem mirrors.

Here in Appendix B we present a full three-dimensional analysis of "large- $n$ " ballooning modes in the axicell configuration of MFTF- $B$. Since this analysis was performed, the proposal coil set for MFTF-B has been altered. While the resulting changes in the equilibrium magnetic field can be expected to change quantitative results, we do not expect a change in our main qualitative result: in a machine the size of MFTF-B $\left(a_{i} / R_{p}=0.06\right.$ in the central cell), localized modes will not limit the central-cell $\mathrm{B}$.

In Sec. 2 we discuss flute stability, and in Sec. 3 we review the "large-n" formalism" ${ }^{B-2}$ as it applies to tandem mirrors. In Sec. 4 we present the ideal MHD central-cell beta limit due to large-n ballooning modes. In Sec. 5 we show how the formalism of "quasi-classical" mechanics may be used to obtain a global-dispersion relation, which includes both $k_{\text {inet ic effect }}^{\mathrm{B}-3,4}$ 
and the " $1 / \mathrm{n}$ " corrections. . $^{\mathrm{B}-3}$ We present marginal-stability boundaries for the axicell configuration of MFTF-B in Sec. 6 and discuss our results in Sec. 7 .

\section{FLUTE STABILITY}

In the low-B limit, the MHD eigenmodes of a tandem mirror are flute-like. B-1 A sufficient condition for the instability of such modes is that

$$
\int_{-L}^{L} \frac{d z}{B} \frac{\partial}{\partial \psi} p \rho>0, p=\frac{p_{1}+p_{11}}{2},
$$

where $B$ is the magnetic-field strength $(B=\hat{B} b) ; \Psi$ is the principal flux coordinate $(\underset{\sim}{B}=\underset{\sim}{\nabla} \times \underset{\sim}{\nabla \theta}) ; P_{1, \|}(\psi, B)$ are components of the plasma-pressure perpendicular and parallel to $B$; and $G$ is the normal curvature $(\underline{K}=\hat{b} \cdot \underset{\nabla}{\mathbb{D}}=\vec{F} \psi+F \nabla \theta)$. Thus, it is necessary for stability that the flux-tube average of the pressure-weighted normal curvature be positive.

This condition along with minimization of plasma current flowing parallel to $B$ through the central cell have been the major constraints on the design of the MFTF-B magnetic-field coils. While the design continues to evolve toward one with acceptably small central-cell parallel current, we examine the flute stability of our latest design, which has not yet satisfied all constraints. We consider two pressure models for the transition region; one in which the pressure is constant $(B \approx 0.05)$ and the other in which $p \sim B$ represents a passing and trapped population with $a g_{B} \approx 3$. Stable central-cell beta-yalues $\left(B_{c}\right)$ are listed in Table $B-1$ for both equilibrium and vacuum-field configurations for an axicell beta of 0.2 and an anchor beta of 0.55 , the reference-case values. These central-cell beta values are to be

Table B-1. Critical beta-values for the central cell.

\begin{tabular}{llll} 
& $\begin{array}{c}\text { Equilibrium } \\
\text { field }\end{array}$ & $\begin{array}{l}\text { Vacuum } \\
\text { field }\end{array}$ & Mars-mode \\
\hline${ }^{P}{ }_{T R} \sim B$ & $B_{C}=0.36$ & $B_{C}=0.6$ & $B_{C}=0.5$ \\
$P_{T R}$ constant & $B_{C}=0.53$ & $B_{C}=0.96$ & $B_{C}=0.5$ \\
\hline
\end{tabular}


compared with the value of 0.5 of the reference case (Mars mode). While the precise value of the critical central-cell $\beta$ for flute stability will probably be somewhat different for MFTF-B, the qualitative result should be the same: flute modes are not expected to impose a serious constraint on performance of the machine.

\section{LARGE-N THEORY AND OTHER APPROXIMATIONS}

In the past few years a great deal of progress has been made in analyzing ballooning modes by employing the "large- $n$ " expansion. ${ }^{B-2}$ Although this formal ism was originally developed to analyze ballooning modes in tokamaks ${ }^{B-2,3}$, it has been extended to non-axisymetric systems like tandem mirrors. ${ }^{8-4}$ The large-n formalism describes modes in which the typical scale length for variation of the perturbation across the magnetic field is short in comparison to either the equilibrium scale lengths or the scale length for variations of the perturbation parallel to B. An eikonal approximation, $\phi \simeq \widetilde{\phi}(s) \exp [i S(\theta, \psi)]$, is then employed to describe the perpendicular variations in the stream function, $\phi$, which describes the perturbation. The assumption of rapid perpendicular variation allows one to reduce the MHD ballooning mode problem to the solution of a second-order ordinary differential equation along each magnetic-field 1 ine. $B-2,3$

This equation is most simply written in magnetic-flux coordinates, $(s, \theta, \psi)$; where the enclosed magnetic flux $\psi$ labels a particular flux surface; $\theta$ is an angle-like variable that labels a particular magnetic field line on this flux surface; and $s$ measures the position along this magnetic-field Iine. In the work reported here, we use the long-thin or paraxial expansion. $B-5$ To lowest significant order in the long-thin parameter, $\lambda=R / L$ ( $R$ is a typical radial dimension and $L$ is a typical axial dimension), no difference exists between $s$ and the axial distance $z$. Hence, we may write the ballooning-mode equation as a second-order differential equation in $z$ :

$$
\begin{aligned}
{\left[\frac{\partial}{\partial z} \frac{Q|\nabla S|^{2}}{B^{3}} \frac{\partial}{\partial z}\right.} & +\frac{Q(z)}{B^{3}}|\nabla S|^{2}\left(\omega^{2}-\omega R^{*} S_{\theta}\right) \\
& \left.+\frac{1}{B^{3}}\left[\nabla\left(P_{\perp}+P_{U}\right) \times \tilde{b}\right](\underset{\sim}{K} \times \hat{b}): \underset{\sim}{\nabla S} \underset{\sim}{\nabla S}\right] \tilde{\phi}=0,
\end{aligned}
$$


where $Q=B^{2}+P_{1}-P_{\| I}$ is the parallel component of the total stress tensor; $^{B-5} \nabla S$ is the gradient of the eikonal $S(\theta, \psi) ; \rho(2)$ is the mass density; $\omega$ is the wave frequency; $\Omega^{*}=\left(T_{i} / e B\right)\left(1 / r R_{p}\right)$ is the anguiar velocity of diamagnetic rotation $\left[R_{p}\right.$ is the scale length for variation in the perpendicular ion pressure, $\left.R_{p}^{-1} \equiv-\left(r B / P_{i_{1}}\right)\left(\partial P_{i_{1}} / \partial \psi\right)\right]$; while $S_{\theta} \equiv \partial S / \partial \theta$ is proportional to the surface component of the wave vector. Hence, $\Omega^{\star} S_{\theta}$ is the usual diamagnetic drift frequency. In writing this equation we have ignored the finite- $\beta$ corrections to $\Omega^{*}$ (see Ref, $8-4$ ). This approximation is justified because we find that $\mathrm{Eq}$. B.2 properly describes the most unstable modes only at modest values of the central-cell beta, $B \leqslant 0.1$.

We will solve this equation subject to the boundary condition $\partial \widetilde{\phi} / \partial z=0$ at the axial boundaries of the plasma. Physically, this boundary condition iollows from requiring that the perturbed current vanish in the vacuum external to the plasma. A consequence of this boundary condition is that, while the labels of magnetic-field lines beyond the axial boundaries of the plasca may be interchanged by the perturbation, the structure of the external magnetic field is left unaltered.

It is necessary to find MHD equilibria in order to evaluate the coefficients in Eq. B.2. Nonaxisymetric tandem-mirror equilibria are obtained by using the expansion described in Ref. B-6. This evolves expanding in both the long-thin parameter $\lambda$ and in $B \equiv 2 P / B^{2}$.

A final approximation is to treat $\Omega^{*}$ as constant, independent of $\theta, \psi$, and $z$. The lack of dependence on $\theta$ and $\psi$ is justified if $\Omega^{\star}$ varies little over the region in $\theta$ and $\psi$, over which the modes are localized. This approximation is analogous to the approximation $\partial u^{*} / \partial r=0$ used in the nonlocal anaiysis of electron-drift waves in toroidal systems. ${ }^{B-7}$ Diamagnetic effects are most important in the long central cell of tandem mirrors. ${ }^{B-8}$ and $\Omega^{*}$ is nearly constant in this region. Hence, we believe that the approximation $\partial \Omega / \partial z$ is justified. He will see in Sec. 6 that the approximation $\delta$ = constant greatly simplifies the system of equations that must be solved numerically to obtain the marginal-stability boundary for ballooning modes in a given magnetic configuration. 
4. IDEAL MHD RESULTS

Previous analysis of beta limits in MFTF-B were based on a study ${ }^{B-9}$ of ideal MHD baliooning modes in the large-n limit. We review the principle results of ideal MHD-ballooning-mode theory here because they provide a basis for understanding the full three-dimensional treatment, including both the $k$ inetic term and the "1/n corrections" described in the next section. The ideal MHD-ballooning-mode equation may be obtained from Eq. B.2 by taking the limit $\Omega_{*}+0$. Since $\Omega^{*}$ is proportional to $a_{j} / R_{p}$, this is equivalent $t_{0}$ considering a very large machine, in which the ion gyroradius $a_{i}$ is very much less thar a radial-scale length $R$. In the ideal MHD limit, each term in the ballooning-mode equation is proportional to $|\nabla S|^{2}$. Upon dividing the equation through by the value of $|\nabla S|^{2}$ at the midplane of the central cell,

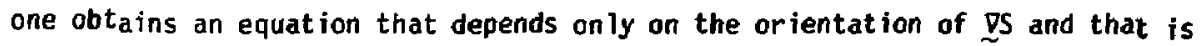
independent of the magnitude of VS. Hence, Eq. B.2 can provide no information about the magnitude of DS.

The orientation of DS is determined by

$$
\Gamma \equiv 2 \psi S_{\psi} / S_{\theta}
$$

The marginal stability condition for ideal MHD ballooning modes in nonaxisymetric tandem mirrors depends on both the field-line labels, $(\theta, \psi)$, and on $\Gamma$. At small to moderate values of $\beta_{c}$, the "worst" field line (i.e., the last field line on which ideal MHD-ballooning modes are unstable as $\beta_{c}$ is decreased) a lways occurs at $\theta=N \pi / 2$. These field lines lie on one of the principle axes of the elliptical flux surfaces in and neir the HHO anchor ceilis. The stability properties are the same ror any va iue of $N$ as a result of quadrupole symmetry. Hence, we need only consider MHD stability in the neighborhood of $\theta=0$.

The worst value of $\psi$ generally iies about midway out in the radial profile, which is Gaussian for the assumed constant $\Omega^{*}$. Figure B-1 shows the critical value of $B_{C}$ for marginal stability in the axicell configuration of MFTF- $B$ vs $\psi$. The peak value of beta in the MHD anchor cell is held fixed at $B_{a}=0.55$, while the peak value of beta in the axicell (where the central-cell jons are plugged by the ambipolar potential) is set at $\beta_{p}=0.11$. Values of $\theta$ and $\Gamma$ have been chosen at each value of $\psi$ to minimize $B_{c^{-}}$. We see that the 


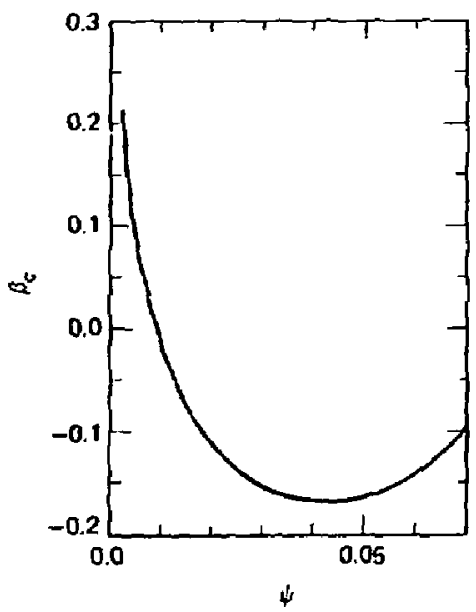

Fig. B-1. Critical value of $B_{c}$ as a function of $\psi$. The values of beta in the MHD anchor cell and the axisymmetric plug cell are held fixed at $B_{a}=0.55$ and $\beta_{p}=0.11$. Theta and $\Gamma$ were chosen at each value of $\psi$ to minimize $\beta_{c}$.

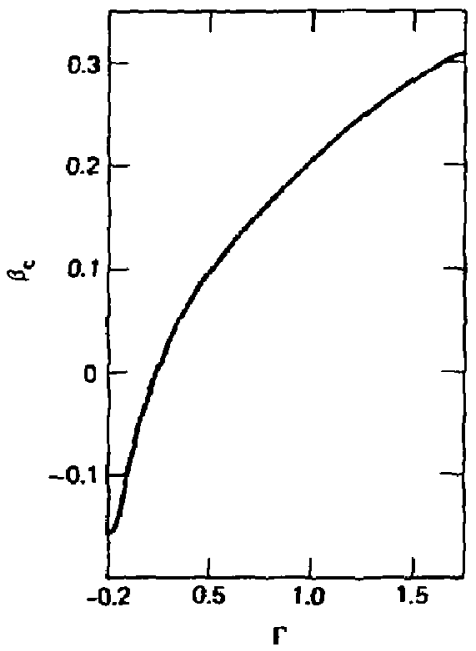

Fig. B-2. Critical value of $B$ vs $\Gamma$ for $B_{a}=0.55, B_{p}=0.11$, and $\theta=0 ; \psi$ is held fixed at $\psi / \psi_{\text {edge }}=0.043$. 
worst flux surface at $\psi=0.043$ ljes at the bottom of a quite gentle well in critical $B_{\mathrm{c}}$.

Similarly, in Fig. B-2 we show the critical value of $\beta_{c}$ vs $\Gamma$ for the same values of $B_{a}$ and $\beta_{p^{*}}$. In fig. $B-2, \theta$ is held fixed at zero and $\psi$ is fixed at $\psi / \psi_{\text {edge }}=0.43$, the worst field 1 ine for $\Gamma=0$. We see that worst orientation, $\Gamma=0$, lies at the bottom of a very steep we 11 in $B_{c}$. When $\Gamma$ is less than about 0.25 , we see that the critical value of $\beta_{c}$ is negative. That is, the axicell configuration of MFTF-B is found to be unstable to ideal MHD-ballooning modes even at zero central-cell beta. Increasing $B_{a}$ makes the system more stable, while increasing $B_{p}$ makes the system more unstable.

We find in Sec. 6 that this result is far too pessimistic. When effects associated with the perpendicular structure (i.e., "1/n corrections") and diamagnetic drifts (i.e., "kinetic terms") are included, the beta 1 imit is great $y$ increased and falls somewhere in the range $0.1 \leq \beta_{c} \leq 0.4$. This result, first, is in part due to the stabilizing kinetic term, which acts over the entire axial length of a tanden mirror, while the destabilizing pressure-curvature term acts only in the axicell and the transition region between the axicell and the MHD anchor cell. Second, the result is in part due to the " $1 / n$ corrections"; that is, the orientation of the perpendicular wave vector, as described by $\Gamma$, is determined by the perpendicular structure of the mode and is no longer free to be adjusted so that it $f$ its into the deep well in Fig. $B-2$ near $\Gamma=0$.

\section{METHOD OF SOLUTION}

The stabilizing kinetic effects enter Eq. B.2 through a term proportional to $S_{\theta^{*}}$ Unfortunately, the single-field-line problem provides no information about the magnitude of $S_{\theta}$; this information must be obtained from a solution of the eigenvalue problem in the plane perpendicular to B. This is in contrast to the ballooning-node problem in axisymmetric systems, like tokamaks, where $S_{\theta}$ is replaced by the toroidal-mode-number $n$. In axisymmetric systems $n$ is a constant, so one can independently analyze the kinetic term and the radial-mode structure, which leads to the $" 1 / n$ corrections". B-3 In nonaxisymmetric systems, like the axicel1 MFTF-B configuration, these two problems are coupled through $S_{\theta}$, so that they must be treated together. 
Equation $B .2$ describes the behavior of large-n (i.e., $S_{\theta} \gg 1$ ) ballooning modes on each magnetic-field line. A numerical solution of this equation along a particular field line yields the local dispersion relation,

$$
D\left(\theta, \psi, \Gamma, \Omega^{2} ; \beta_{c}, \beta_{a}, \beta_{p} \ldots\right)=0 \text {. }
$$

The explicit dependence on $\mathrm{S}_{\theta}$ enters through the parameter

$$
\Omega^{2} \equiv \omega\left(\omega-s_{\theta}\right) \text {. }
$$

Note that the local dispersion relation depends not only on the field-line labels $(\theta, \psi)$, the wave parameters $\$ S$, and $\omega$, but also on the equilibrium parameters $8 *, \beta_{c}$, etc.

Our problem is to "sew" together the solutions on each field 1 ine in a self-consistent way to obtain both the mode structure in the $(\theta, \psi)$ plane and the global dispersion relation. We accomplish this by using the theory of quasi-classical mechanics--essentially WKB theory generalized to many dimensions. ${ }^{8-10}$ In the quasi-classical formalism, the local dispersions relation 0 may be viewed as the Hamiltonian governing the motjon of a ray in the four-dimensional phase space: $\left(\theta, \psi, S_{\theta}, s_{\psi}\right)$, where the field line labels $(\theta, \psi)$ are the coordinates and the covariant components of $\nabla S,\left(S_{\theta}, S_{\psi}\right)$, are the conjugate momenta. The ballooning mode ray then obeys the equations of motion

$$
\begin{aligned}
& \dot{\theta}=\frac{\partial D}{\partial S_{\theta}}, \\
& \dot{\psi}=\frac{\partial D}{\partial S_{\psi}}, \\
& \dot{S}_{\theta}=-\frac{\partial D}{\partial \theta},
\end{aligned}
$$

and

$$
\dot{S}_{\Psi}=-\frac{\partial D}{\partial \psi}
$$


The ray motion described by Eqs. B.6-d may be either integrable or stochastic. ${ }^{B-11}$ The Hamiltonian $D$ is clearly a constant of the ray motion since the ray must stay on the surface $D=0$. If there is a second independent constant of the motion, then the ray orbit must lie on a two-dimensional surface, $\Sigma$, embedded in the four-dimensional phase space. It may be shown that the surface $\Sigma$ is topologically a torus. This surface is central to the quasi-classical theory of mechanics, where it is known as the "invariant torus". Ray orbits that lie on an invariant torus are said to be integrable.

If a second constant of motion does not exist, then the ray orbit fills a three-dimensional region of phase space (the energy shell), and the ray orbit is said to be stochastic.

The theory of quasi-classical mechanics is concerned primarily with integrable systems. If the ray orbits are stochastic, little can be said about the mode spectrum; while if the ray orbit is integrable, then the dispersion relation may be obtained by quantizing the two independent actions,

$$
I_{k}=\oint_{c_{k}} \nabla s \cdot d q
$$

These two independent actions are obtained by following a closed path that goes either once around the invariant torus the short way $\left(C_{1}\right)$ ar else once around the torus the long way $\left(C_{2}\right)$. This is illustrated in Fig. B-3. The quantization condition for the systems considered here is

$$
I_{k}=\left(2 n_{k}+1\right) \pi
$$

We $f$ ind that the mades associated $w i$ th the larger values of $n_{k}$ are more stable than the $n_{k}=0$ modes. Hence, in studying marginal stability one need only consider normal modes satisfying

$$
\mathbf{I}_{\mathbf{k}}=\pi \text {. }
$$

Before attempting to apply the methods of quasi-classical mechanics we must determine if the ray orbits associated with "large- $n$ " ballooning modes in the axicell configuration of MFTF-B are integrable. This is accomplished by a direct numerical integration of the ray equations of motion. A ray is initialized with a particular set of phase variables $\left(\theta_{0}, \Psi_{0}, S_{00}\right)$. The fourth phase variable, $S_{\psi 0}$, is chosen such that the ray lies on the "energy she11", $0=0$. The orbit is advanced in time using Eqs. (B.6) together with 


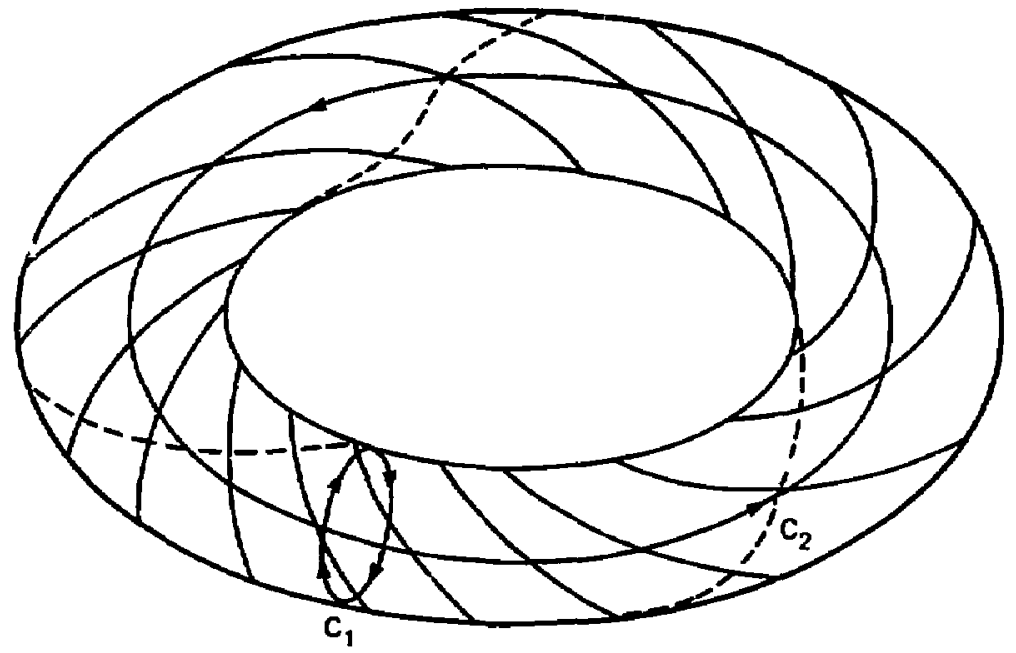

Fig. B-3. Phase-space trajectory and invariant toroid for two degrees of freedom. The $C_{1}$ and $C_{2}$ curves are for defining the action integrals $I_{1}$ and $I_{2}$. The toroidal helix is the trajectory; normally it is not closed. (Shown with permission of I. C. Percival, Ref. B-10.) 
the Livermore Solver for Ordinary Differential Equations (LSODE). B-12 At each time step, the ballooning-mode equation B.2, is integrated on the current field line to calculate $D$ and its derivatives. The value of $D$ is monitored to check the accuracy of the integration. Integrability may then be determined from a Poincaré map; each time that the ray passes through the hyperplane $\psi=\psi_{0}$, the current values of $\theta$ and $S_{\theta}$ are plotted. If the ray arbit is integrable, then these points will lie on a smooth curve as in Fig. $B-4$, while if the ray orbit is stochastic then they will fill an area in the $\left(\theta, S_{\theta}\right)$ plane. Figure B-5 shows a stochastic orbit. We did not follow this orbit long enough to see this area-filling property in the Poincaré map. We find that the ray orbits are of ten but not always integrable. When the equilibrium parameters are in the general vicinity of ideal MHD marginal stability, the ray orbits are found to be integrable; while as we move further from ideal MHD marginal stability (by increasing $\beta_{c}$ for example), this integrability breaks down. The assumption that $S_{\theta} \gg 1$ also breaks down for the most unstable modes far from ideal MHD marginal stability, so that this loss of integrability does not by itself limit our calculation.

It is really somewhat remarkable that any ray orbits are found to be integrable, as it is an unfortunate fact of classical mechanics that most twodimensional Hamiltonian systems are not integrable. Two general cases exist in which two-dimensional systems become integrable. Either there is a symmetry (perhaps a hidden one) or there is a separation of time scales. Our systam is an example of one in which there is a separation in characteristic time scales of the ray motion. This may be seen in the projection of the ray orbit into the $(\theta, \psi)$ plane shown in Fig. B-4. There is a rapid motion directed generally parallel to $\not \Psi$ superimposed on a slow precession in $\theta$. We believe that this separation of time scaies is associated with the extreme ellipticity of the equilibrium flux surfaces in the transition region between the MHD anchor cell and the axice11. Ballooning modes are associated with the bending of fiux bundles in this transition region. When a flux bundie with a circular cross section in the central cell is mapped into this region, it also takes on an elliptical cross section. These elliptical flux bundles act much like pieces of tin; they are easily bent perpendicular to the flat plane but are very stiff when bent parallel to the flat plane. Hence, the ballooning ray has a rapid quiver associated with bending the elliptical flux bundles parallet to their major axis in the transition region. The action associated 

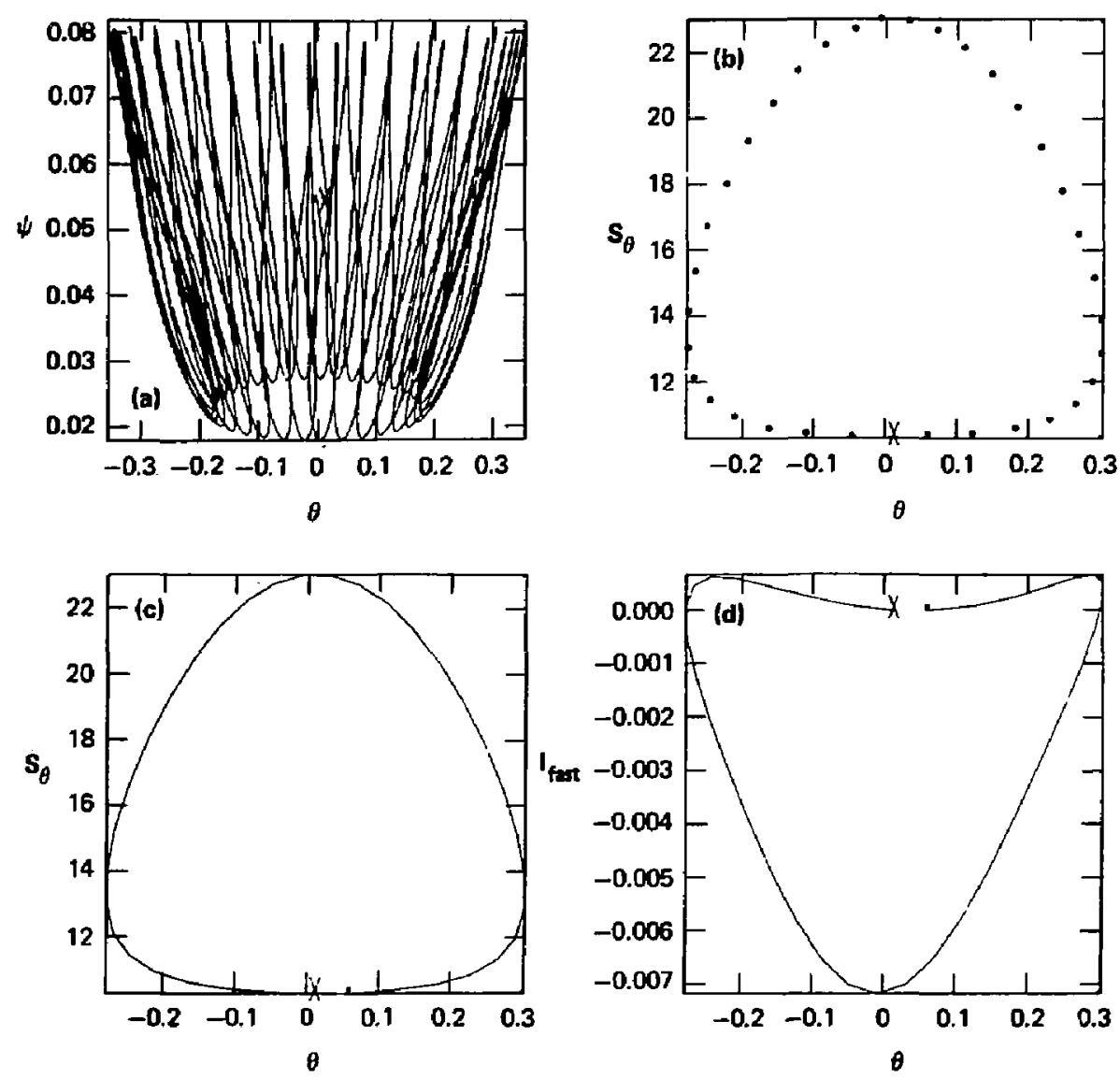

Fig. B-4. Example of an integrable ballooning ray orbit: (a) Projection of the ray orbit onto the $\psi, \theta$ plane; (b) surface of section plot of $S_{\theta} v s \theta$; (c) points connected to form a smooth curve; and (d) our computed value at $I_{\text {fast: }}$ which is shown to be well conserved. 

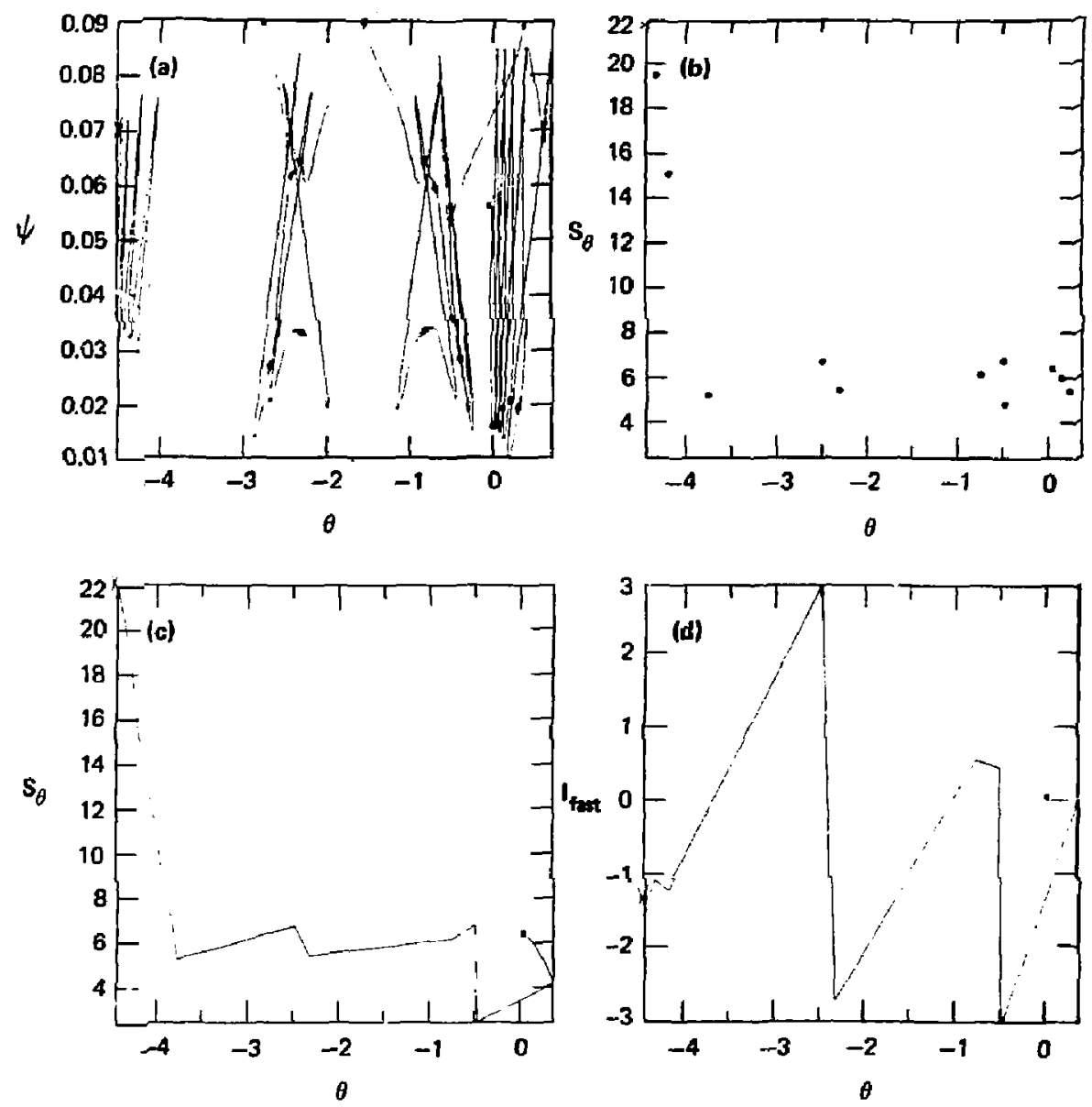

Fig. B-5. Plots same as in Fig. 8-4 except for a stochastic orbit. 
with this rapid motion, $I_{\text {fast, }}$ is then an adiabatic invariant over the slow motion. Hence, $I_{\text {fast }}$ is the second independent constant of motion that guarantees the integrability of ray orbits.

A numerical approximation to $I_{\text {fast }}$ may be obtained by following the ray orbit once around the torus the short way, accumulating fS - dg along the ray orbit and then closing the loop by using a two-point, Simpson's rule integration to step back to the initial point along a line of constant $\psi$. This scheme allows us to evaluate $I_{\text {fast }}$ once for each point on the Poincare map. The numerically determined value of $I_{f} t^{-\pi}$ is plotted in Figs. B-4 and B-5. The initial phase variables were chosen such that $I_{f}$ ast $=\pi$ for the first loop so that the dispersion relation, Eq. B.9, is satisfied. He see from Figs. $B-4$ and $B-5$ that $I_{\text {fast }}$ is indeed we 11 conserved on the integrable orbit, while it is not conserved on the stochastic orbit.

The separation of time scales also provides us with some information about the frequency spectrum. The separation in frequency between modes with neighboring values of $n_{\text {fast }}$ is $\Delta \omega \sim 2 \pi / T_{\text {fast }}$, where

$$
\begin{aligned}
\mathrm{T}_{\text {f ast }} & =\frac{\partial}{\partial \omega} \mathrm{I}_{\text {fast }} \\
& =\oint_{d g} \cdot \frac{\partial}{\partial \omega} \nabla S,
\end{aligned}
$$

and the integral is to be taken once around the torus the short way. Noting that $\left|\partial D_{S} / \partial_{i, j}\right|$ may be interpreted as $1 / v_{\text {group }}$, we see that $\mathrm{T}_{\text {fast }}$ is essentially the period of the fast motion. Similarly, the frequency separation between modes with neighboring values of $n_{\text {slow }}$ is $\delta w \sim 2 \pi / T_{\text {slown }}$ where

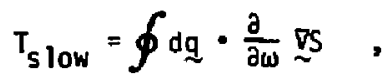

with the integral taken once around the torus the long way ( $I_{\text {slow }}$ is essentially the area enclosed by the line segments connecting the points on the Poincare map in Fig. B-4). Hence,

$$
\frac{\delta \omega}{\Delta \omega} \sim \frac{T_{\text {f ast }}}{T_{\text {slow }}} \ll 1 .
$$

That is, the line spectrum must be like that shown in Fig. B-6. 


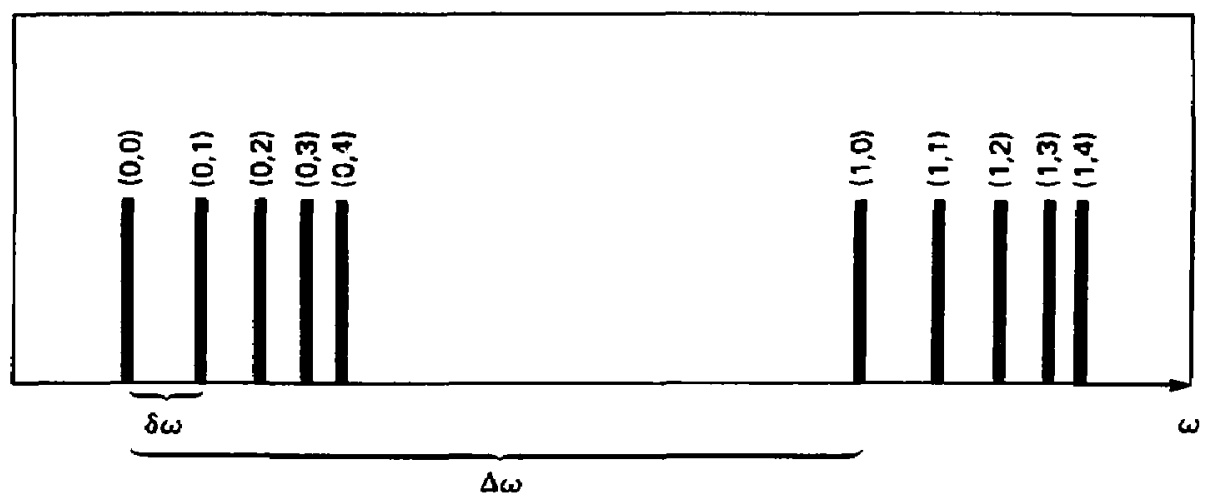

Fig. B-6. Sketch of line spectrum that is characteristic of a system with well-separated time scales. Each normal-mode frequency is 1 abeled by the EBK mode number ( $n_{\text {slow' }} n_{\text {fast }}$ ). 
It follows that we need only do a careful job in quantizing $I_{\text {fast }}$, while the remaining parameter that determines $I_{s}$ low may be chosen to maximize instability. This will put us within dw of the most unstable mode. Instability is maximized when the derivative of $I_{\text {fast }}$ with respect to the remaining parameter vanishes. This approximation is analogous to estimating the dispersion relation in a plasma slab by using local theory at the most unstable value of the inhomogeneous coordinate. This procedure generally does reasonably we11. although $i:$ misses WKB corrections (which are usually stabilizing) that are ussociated with the radial-mode structure. In the present instance, we retain WKB corrections associated with the fast motion but ignore small stabilizing corrections associated with the slow motion. This is an important practical simplification because it is now only necessary to follow a ray once around the torus the short way and compute just $I_{\text {fast }}$. This in turn requires on the order of $10^{3}$ integrations of the balloon equation and takes several seconds of CPU time on a CRAY-1. A numerical computation of $I_{\text {slow }}$ (which we are avoiding) would require that we follow the ray for at least one full period of the slow motion. This requires between five and ten minutes of CPU time on a CRAY-1. Hence, ignoring corrections to the mode frequency of order $\delta \omega$ as compared to corrections of order sw saves hours of computer time in computing marginal stability boundaries.

\section{MARGINAL STABILITY BOUNDARIES}

It is necessary to find the invariant torus associated with the unstabie "large $-n^{*}$ ballooning modes to obtain the dispersion relation and the marginal stability boundary. We expect that the most unstable ballooning modes will be localized in the neighborhood of the worst field line of ideal MHD theory. Hence, we may fix $\theta_{0}$ and $\psi_{0}$ as the labels of this field line. The initial value of $S_{\theta}, S_{\theta 0}$, then selects a particular invariant torus from among those intersected by the curve

$$
\begin{aligned}
& \theta=\theta_{0} . \\
& \psi=\psi_{0}, \\
& D\left(\theta, \psi, \Gamma, \Omega^{2}\right)=0 .
\end{aligned}
$$


Given a particular set of equilibrium parameters, the frequency of the most unstabie mode satisfies the equations

$$
I_{\text {fast }}\left(\theta_{0}, \psi_{0}, S_{60}, \Omega^{2} ; B_{c}, \ldots\right)=\pi
$$

and

$$
\frac{\partial I_{\text {fast }}}{\partial S_{\theta 0}}=0
$$

If we require this mode to be marginally stable, we must satisfy the additional equation

$$
\frac{\partial I_{f a s t}}{\partial w}=0
$$

In general, it would be necessary to simultaneously solve Eqs. B. 14 to B.16. Fortunately, the assumption $\Omega^{*}=$ corstant, together with the fact that $S_{\theta}$ has only a small fractional variation during one period of the rapid motion allows us to replace the third equation with the condition

$$
\frac{\partial \Omega^{2}}{\partial \omega}=0
$$

or, from Eq. (B.5),

$$
\omega=\frac{\Omega \star S_{00}}{2}
$$

This results in a considerable simplification, as it is now only necessary to solve Eqs. B. 14 and B. 15 simultaneous $1 y$ with $\Omega^{2}$ set equal to $-\left(\Omega+S_{\theta 0} / 2\right)^{2}$.

These equations may be solved numerically to find the marginally stable value of $\Omega^{\star}$ for a particular set of equilibrium parameters, $B_{c}, B_{a}, B_{p} \ldots$. Then one of these parameters, say $\beta_{c}$, may be varied to produce a stability boundary like that shown in Fig. B-7. We are able to track the stability boundary from ideal MHD marginal stability at $B_{c}=-0.167$ to $B_{c}=0.1$. At this point an examination of the ray orbit shows that, while the orbit performs several rapid oscillations, it ultimately escapes like the ray orbit shown in Fig. B-5. Hence, the ray orbit is no longer integrable, and the quasi-classical procedure outlined in Sec. 5 is no longer justified. As long 


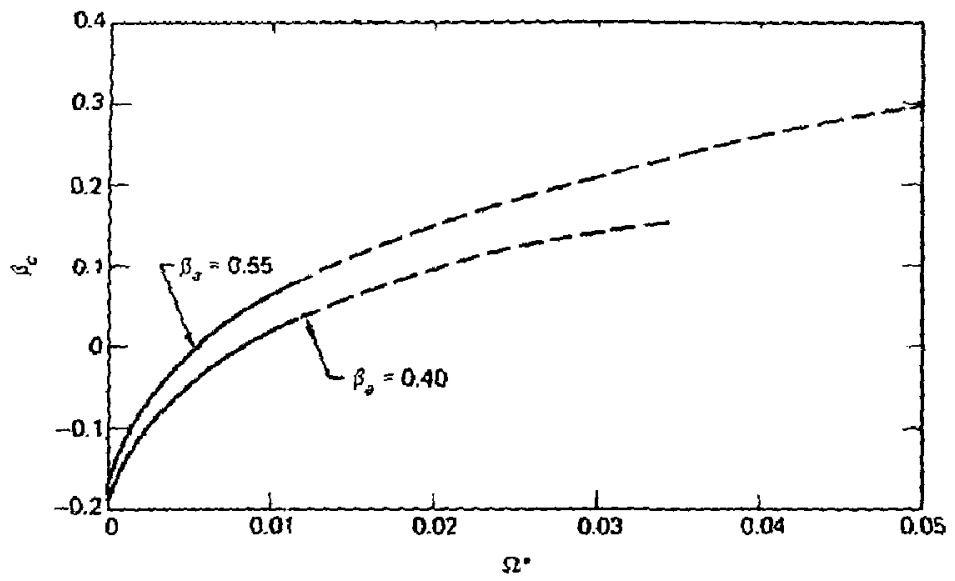

fig. 8-7. Marginal stability boundary in the $B_{C}$, $\Omega^{*}$ plane. The projected operating point with this coil set is $\Omega^{\star}=0.1$. The value of beta in the axicell is $B_{p}=0.11$, while the $M H D$ anchor-cell beta is $B_{a}=0.55$ (upper curve) or 0.40 (1ower curve). The flute average-beta limit for $B_{a}$ is 0.55 , as shown by heavy line near $B_{c}=0.4$.

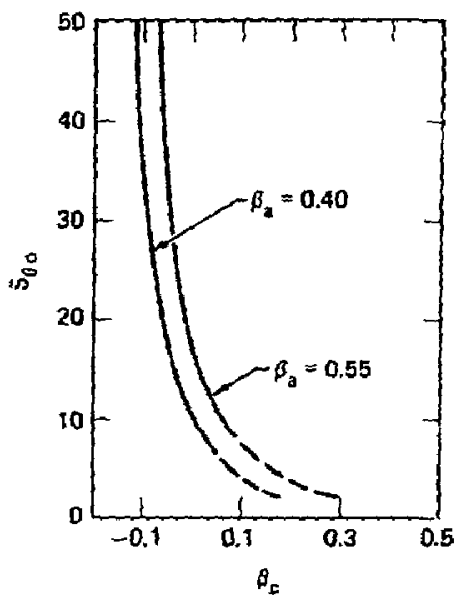

Fig. B-B. A plat of $S_{\theta}$ ys $B_{c}$ at marginal stability. 
as the ray orbit continues to perform even one rapid oscillation it is passible to define $I_{\text {fast }}$ and to solve Eqs. B.15 and B.16. Points on the marginal-stability boundary obtained in this manner are connected by a dashed line in Fig. B-7.

Figure $B-8$ shows the values of $S_{00}$ on the marginal-stability boundary. We see that the condition $S_{\theta} \gg 1$, required in the derivation of Eq. (B.2), also breaks down near $\beta_{c} \simeq 0.1$. Hence, there is little significance to the dashed portion of the marginal-stability curve.

The effect of varying beta in the MHD anchor cell, $B_{a}$, is shown by the lower curve in Fig. B-7, in which $B_{a}$ has been reduced from 0.55 to 0.40. Just as in ideal MHO ballooning-mode theory, we find that decreasing $B_{a}$ tends to destabilize the system.

\section{SUMMARY}

The main result of this calculation is the marginal-stability boundary shown in Fig. B-7. An important feature of the marginal-stability boundary is the fact that the characteristic value of $S_{\theta}$ at the marginal stability decreases as $B_{C}$ and $\Omega$ increase (see Fig. B-8) unti] the calculation breaks down at $B_{c}=0.1$. At this point, $\Omega^{*}=1.4 \times 10^{-2}$ and $S_{\theta}=7.27$. It is possible to extend the calculation to Targer values of $\beta_{c}$, as indicated by the dashed curves in Figs. $\overline{8}-7$ and $B-B$, but two assumptions underlying this calculation $\left(S_{\theta} \gg 1\right.$ and integrability of the ray orbits) are not satisfied in this regime.

The axicell configuration of MFTF-B lies off to the right of Fig. B-7 at $\Omega^{\star} \simeq 0.1$ in our units. It is clear from Figs. $B-7$ and $B-B$ that "iarge $-n^{\text {" }}$ ballooning modes will not limit the central-cell beta at these large values of s*. The rapid decrease in $S_{\theta}$ on the marginal-stability boundary suggests that at larger values of $\beta_{c}$ and $\Omega^{\star}$, the most dangerous modes will be low-m modes with a global structure in the plane perpendicular to $\underset{B}{\text {. }}$. The beta 1 imit impused by such modes is certainly bounded from above by the flute average-stability condition, $B_{c}<0.4$, as this 1 imit is found to be relatively insensitive to the field-line labels, $(\psi, \theta)$. Hence, we may expect the central-cell beta limit to fall in the range $0.1 \leq B_{c} \leq 0.4$. 
The extension of large-n theory beyond its domain at validity gives a marginal-stability curve (the dashed 1 ine in Fig. B-7) that appears to asymptote to the flute-stability limit as increases. This suggests that at the rather large values of $\Omega$ in the axicell configuration of MFTF- $B$ $\left(\Omega^{*}=0.1\right)$ the actual central-cell beta limit will be closer to the flute average 1 imit of 0.4 . Theoretical and computational studies of the low-m modes that will determine this $B$ limit are currently in progress. 


\section{REFERENCES}

B-1. L.S. Hal1 and B. McNamara, Phys. Fluids 18, 552 (1975).

B-2. J.W. Conner, R.J. Hastie, and J.B. Taylor, Phys. Rev. Letters 40, 396 (1978); A.H. Glasser, in Praceedings of the Finite Beta Theory Workshop, Varenna, Italy, Sept. 1977, B. Coppi and W. Sadowski, Eds. (National Technical Information Service, Springfield, VA., 1979) p. 55.

B-3. R.L. Dewar, J. Manickan, R. C. Grimm, and M. S. Chance, Nuc lear Fusion 21, 493 (1981).

B-4. W.M. Tang and P.J. Catto, Phys. Fluids 24, 1314 (1981); X.S. Lee and P.J. Catto, Phys. Fluids 24, 2010 (1981).

B-5. W.A. Newcomb, J. P lasma Physics 26, 529 (3981).

B-6. L.D. Pearlstein, T.B. Kaiser, and W.A. Hewcomb, Phys. Fluids 24, 1326 (1981).

B-7. See, for example, W.M. Tang, Nucl. Fusion 18, 1089 (1978).

B-8. D.E. Baldwin, Tandem Mirror Geometry Enhancement of FLR Effects, Lawrence Livermore National Laboratory, personal comunication (1980).

B-9. T.B. Kaiser, in Physics Basis for MFTF-B, Ed. by D.E. Baldwin, B. G. Logar, and T. C. Simonen, Lawrence Livermore National Laboratory, Rept. JCID-18496, Part 2 (1980), Sec. IV-8.

8-10. See, for example, 1.C. Perciva7, Adv. in Chem. Phys. 36, 1 (1977).

B-11. See, for example, 8.V. Chirikov, Phys. Reports 52, 263 (1979).

B-12. A.C. Hindmarsh, Linear Multistep Methods for Ordinary Differential Equations, Lawrence Livermore Nationa] Laboratory, Rept. UCRL-51186 (1972). 


\section{APPENDIX C}

SOME EFFECTS OF HOT-ELECTRON STABILITY IN TANDEM-MIRROR GEOMETRY

\section{E. Baldwin}

A hot, anisotropic electron component will continue to play an important role, it appears, in tanden-mirror (TM) confinement, either as a potentfal-depressing component in a thermal barrier or as a pressure-enhancing component in an magnetohydrodynamic (MHD) anchor. The theory of the interactions of these hot electrons with low-frequency interchange and ballooning modes follows closely that of Elmo Bumpy Torus (EBT) stability, but some features are special to the TM geometry. In this note, some of these special features are examined by using a model balloon-mode equation.

The most immediate special feature of $T M$ 's are the several axial regions having particles of quite differenc energies and, therefore, different or ift speeds. He model this by treating each region as a localized slab having magnetic curvature simulated by a local effective gravity. These regions are then coupled by a balioon-mode equation obtained by setting $\nabla \cdot j$ (perturbed) $=0$. We introduce the curvature-drift frequency of and the $\nabla B-d r i f t$ frequency $\omega_{B}$ for each species and assume that $B$ exceeds the ratio of the plasma-density scale length $r_{n}$ to the magnetic radius of curvature $R_{c}$. Then for each species $w_{B} \gg w_{k}$. Hot-species quantities are labeled by subscript "h", and warm-species quantities by "w", with hot and warm defined by drift speeds relative to wave frequency in the ordering $\omega \gg \omega_{\mathrm{Bw}} \gg \omega_{k w}$ and $\omega_{B h} \gg \omega \imath \omega_{k h}$.

The balloon equation for eikonal solutions in finite Larmor radius (FLR) ordering and this frequency ordering is given by

$$
B \frac{d}{d s}\left(\frac{k_{\perp}^{2} Q}{B} \frac{d \phi}{d s}\right)+\frac{k_{1}^{2}}{v_{A}^{2}}\left[\omega\left(\omega-\omega_{\star_{i}}\right)+\frac{\omega-\Lambda \omega_{k h}}{\omega-\omega_{k h}(1+\Lambda)} \sum \frac{\omega_{k_{j}} \omega_{k}}{b}\right] \phi=0 \text {, }
$$

where $\phi$ is proportional to the displacement; $Q=B^{2}+p_{\perp}-D_{\|} ; V_{A}$ is the local Alfvên speed; $\omega_{* j}$ is the diamagnetic drift frequency; $\alpha$ is the local species-density fraction; $b=1 / 2 k_{\perp}^{2} a^{2}$ with "a" the species gyroradius; $\Sigma$ is the sum over all species; $\sum_{w}$ is the sum over oniy warm species; and 


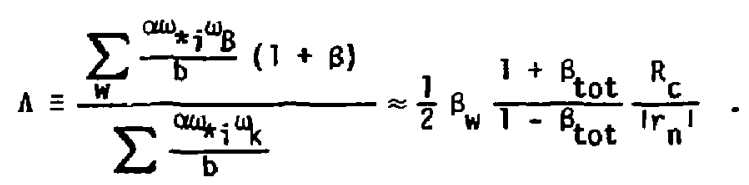

The $w_{t_{i}}$ term is due to finite ion gyroradius. Beinc nonvanishing over the entire solenoid, its effect is enhanced for long solenoids to the point that on $1 y$ low-m modes are unstable. We model $m=1$ by setting $\omega_{\star_{j}} \rightarrow 0$ and taking $k_{1} \approx r_{n}^{-1}$. When $\omega \gg u_{k}$, Eq. $C .1$ then reduces to the familiar MHD balloon equation.

The paraneter $L$, which has the sign of the local curvature, is that obtained by Van Dam and Le ${ }^{\mathrm{C}-1}$ in their analysis of EBT stability. When $w_{k h} \gg w$, the drive เarm

$$
\frac{\Lambda}{T+\Lambda} \sum \frac{a w_{k i} w_{k}}{b}
$$

is locally stabilizirg (i.e., < 0 ) in a well having $w_{* i}{ }_{k}>0$ only when $-1<\Lambda<0$. When $I A$, the denominator of $\Lambda$ replaces the $\Sigma$-drive by the $\sum_{W}$-numerator of $\Lambda$. Tr is latter term describes the warm species moving in the VB-well dug by the hct electron (through $\omega_{B}$ ); the hot electrons otherwise drop out of the problem, is in the "rigid ring" description. If $|\boldsymbol{A}|>1$, the hot electrons respond as MHO fluid in the negative-curvature well.

In the positive-curvature MHD anchor of a $T M, A$ (now $>0$ ) is again important when most of the anchor $\beta$ appears in hot electrons. Again, only the portion $M /(1+\Lambda)$ of the total pressure contributes; or equivalent $y$, orly the anchor $\beta$ excluding hot electrons contributes, although it does so in the enhanced well dug by the hot electrons.

A second class of modes can appear near the hot-electron-drift frequency in a well having $\Lambda<0$. Berk and Domingue ${ }^{C-2}$ show that when $u_{k h}<\Omega_{c i}$, the ion gyrofrequency, stability in a slab requires

$$
\frac{\eta_{h}}{n_{\text {total }}} \leqslant \frac{j}{\frac{k h}{n_{c i}}} .
$$

This originates fron a condition in which there is enough ion mass to preclude the high-frequency $m$ tions, $\omega \lesssim k_{k h}$.

It has been assumed that in a TM the ions of the central cell will serve the function of eliminating the Berk-Dominguez modes. We find that, a?though 
this is true for a flute mode, a long, TM central cell can actualiy have a destabilizing effect.

To illustrate this for a TM configuration having a central cell and a double-end cell, one of positive and one of negative curvature (such as MFIF-P or TARA) we assume modes that are flute through the entire end region and sinusoidal through the central cell. There will be curvature-driven contributions from the plugs $\left(R_{c}<0\right)$, anchor $\left(R_{c}>0\right)$, and transition $\left(R_{c}<0\right)$ between the central cell and end region. Introducing the frequercy associated with Alfven-wave propagation in the central cell $\omega_{A}=2 V_{A} / L_{c}$, we obtain a dispersion relation by equating logarithmic derivative of $\tilde{\phi}$ at $s= \pm \mathrm{L}_{\mathrm{c}} / 2$ :

$$
\frac{2 \omega}{L_{c} \omega_{A}}\left[\begin{array}{r}
-\tan \omega / \omega_{A} \\
\cot \omega / \omega_{A}
\end{array}\right]=B_{c} \int_{p+a+t} \frac{d s}{B V_{A}^{2}}\left[\omega^{2}+\frac{\omega-\Lambda \omega_{k}}{\omega-(1+\Lambda) \omega_{k}} \sum \frac{\alpha \omega_{k} \omega_{k}}{b}\right],
$$

where $p=p l u g, t=$ transition, and $a=$ anchor. The upper term describes even modes and the lower term, odd modes. With $x=\omega / \omega_{A}, x_{k}=\omega_{k} / \omega_{A}$,

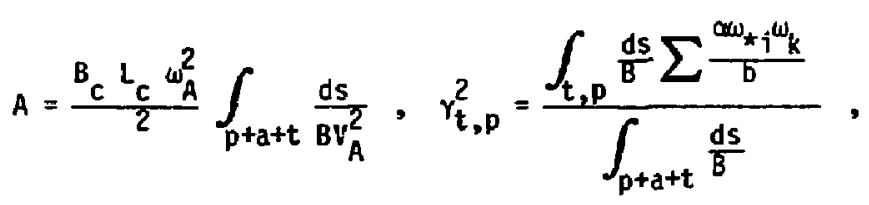

and

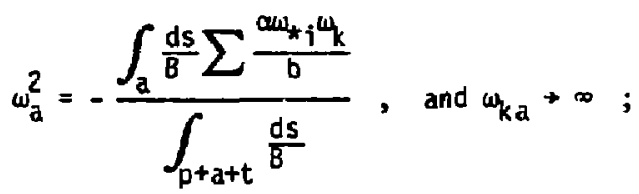

this becomes

$$
\frac{x}{A}\left[\begin{array}{c}
\tan x \\
\cot x
\end{array}\right]=x^{2}+\gamma_{t}^{2}-\omega_{a}^{2} \frac{\Lambda_{a}}{1+\Lambda_{a}}+\gamma_{p}^{2} \frac{x-\Lambda_{p} x_{k p}}{x-\left(1+\Lambda_{p}\right) x_{k p}} .
$$

Here, for simplicity we set $w_{\mathrm{kh}}+\infty$ in the anchor cell.

To analyze rocts of this dispersion reiation, we plot the right and left sides of Eq. C.3 vs $x$, as shown in $\mathrm{Fig} . \mathrm{C}-1$ for the even solution. 


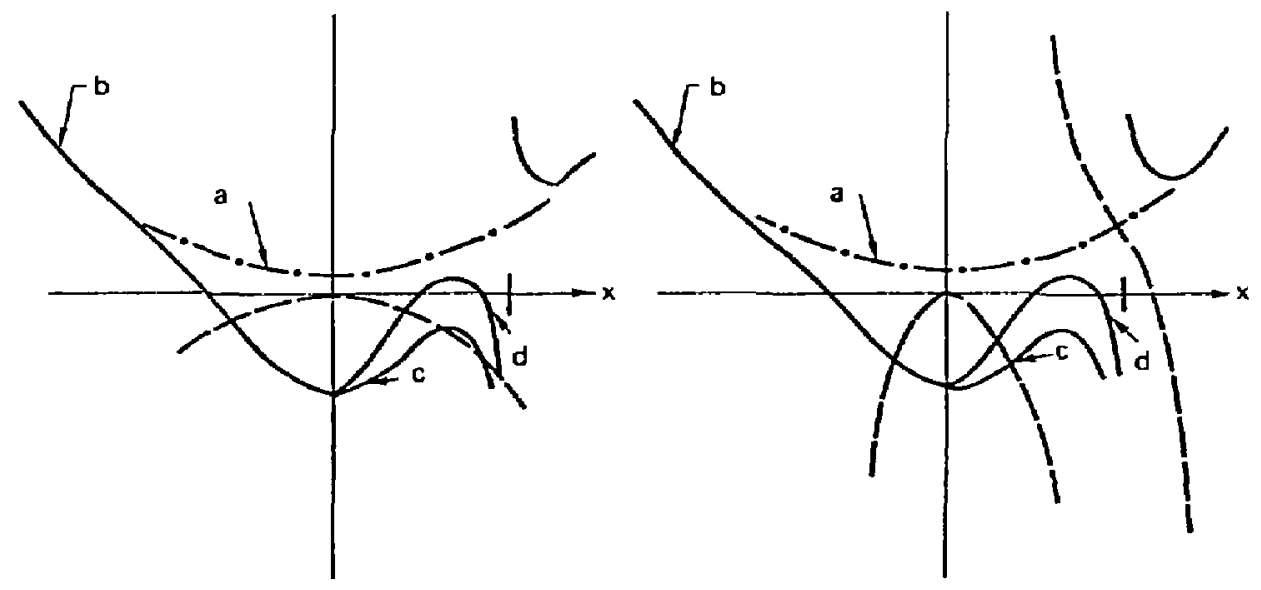

Fig. C-1. Frequency dependence of right side (solid curves) and left side (dashed curves) of the dispersion relation. On the $x$-axis, the mark to the right of the $d$-curve intercept locates the point $x=\left(1+\Lambda_{p}\right) x_{k p}$. See text for explanation. 
Consider first the solid curve alone, which would be the local condition or that appropriate formally as $A+\infty$. Extension "a" of the solid curve is that which would pertain in pure MHD. The dip at $x=0$, generating curve " $b$ ", arises from the plug term at $x=0$. In $E B T$, "a" lies above the axis and stabilization is generated by conversion to " $b$ " by electrons having large $x_{k}$.

Again for $A+\infty$, a second branch of modes is unstable if curve " $C$ " pertains, rather than "d". The condition for "d" is given by Eq. C.2 when $w_{k h}<\Omega_{c i}$. Note that even when local stability is achieved, a negative energy wave exists, as indicated by the negative slope of the solid curve at the root having $\omega>0$.

When the dashed curves are added, the actual roots occur at the intercepts with the appropriate solid curve. When $x_{k} \ll 1$, all of the tan structure is pusned to nigh $x, 1 . e ., x \tan x \rightarrow x^{2}$ as in Fig. t-7. The onily change is a less restrictive Berk/Dominguez condition (e.9., " $c$ " as shown is stable). This corresponds to adding the central-cell-mass weight ing in a flute mode.

Decreasing $\omega_{A}$ by increasing $L_{C}$ pulls the tan structure in until a pair of real roots is lost for $\omega_{k h} \approx \pi \omega_{A^{*}}$. Physically, the otherwise stable negative energy mode in the end cells has coupled to a positive energy shear Alfven wave standing in the central cell to produce instability. Similarly, for a long solenoid, a solution Im $\omega>\omega_{A}$ can be found by setting $\tan x \rightarrow i$.

These modes that originate from the coupling to the solenoid disappear if

$$
\left(1+\Lambda_{p}\right) x_{k p}<0,
$$

which can be achieved either by a good-curvature plug, $\omega_{k}>0$, or by the

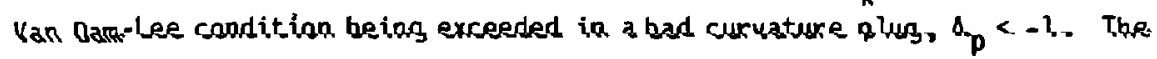
former case is that of TARA; the latter, that of MFTF-B. This result does not bode well for a simple axisymmetric TM stabilized by hot electrons in a bad-curvature wel1; if $\Lambda_{p}<-1$, it would be low-frequency (MHD) unstable, and if $-1<\Lambda_{p}<0$, it would be high-frequency $\left(\omega_{k h}\right)$ unstable.

\section{REFERENCES}

C-1. J. W. Van Dam and Y C. Lee, Proc. Workshop on EBT Ring Physics, Oak Ridge, TN 1979, D. 471 .

C-2. R. R. Dominguez and H. L. Berk, Phys. Fluids 2l, 827 (1978). 


\section{5}


APPENDIX D

ELECTROSTATIC BALLOONING MODES

$$
\text { B.E. Baldwin }
$$

The low-frequency stability analys is described in Appendices $B$ and $C$ was predicated on the assumption that $\tilde{E}_{\| 1}=0$, as in conventional MHD theory. Normally, this condition is well satisfied; an electrostatic field $\partial \tilde{\phi} / \partial s$ drives a parallel current and a subsequent inductive component $\tilde{A}_{\|}$, forcing $\widetilde{E}_{\|}$to zero while introducing bending of the magnetic lines. The energy required for this bending is a stabilizing effect that offsets the tendency of modes to localize in regions of unfavorable magnetic curvature.

The response to $\partial \tilde{\phi} / \partial s$ is quite different for electrons confined in a potential well, as in any open geometry, when their axial bounce frequency exceeds the wave frequency. Then, on successive tounces, they receive nearly compensating impulses on passing through regions of parallel electric fields, and little net paralle 1 current is generated. There results a mode that is substantially flute-like through the solenoid and end-cell regions of unfavorable curvature, and vanishing in regions of favorable curvature. This is an electrostatic (ES) ballooning mode, localized to regions of unfavorable curvature without requiring a bending energy. The transition between the flute-like portion of the perturbation and the region where it vanishes can occur when the equilibrium reflects a significant number of particles, such as a mirror or potential gradient.

In such low-frequency electrostatic disturbances, the electron response is to Debye shield the perturbation, just as in the arialogous situation of electron Debye shielding in an ion acoustic wave. There is a similar parallel ion response when the ion bounce frequency is also large. However, both Debye shielding contributions can be small in geometries having small fractions of particles that pass between regions of good and bad curvature.

We neglect here the axial variations in the electric field drifts and, after transforming to a rotating frame, drop a 11 such drifts. Similarly, because the dominant finite- $B$ effect is to introduce a $\tilde{B}_{\|}$that cancels the magnetic-well effect of the equilibrium $\nabla B-d r i f t$, we consider only electrostatic perturbations in low $B$. Axial variations of both the 
equilibrium $E \times B$ drift and the VB-drift introduce a number of complications whose effects remain to be evaluated, although there do not appear to be any really significant effects arising from these variations.

An eikonal approximation gives the equation for perturbed charge-neutrality,

$$
0=\sum \frac{q^{2}}{m} \int \frac{d \varepsilon d \mu}{v_{11}}\left[\left(\bar{\phi}-\bar{\phi}_{0}^{2}\right) \frac{\partial f}{\partial \varepsilon}-\frac{\bar{\phi}_{0}^{2}}{w-\bar{\phi}_{D}}\left(\Omega^{-1} \underline{k x \underline{b}} \cdot \nabla f+\bar{\omega}_{D} \frac{\partial f}{\partial \varepsilon}\right)\right],
$$

where the sum is over species,

$$
\tau \ldots) \equiv \frac{1}{\tau_{B}} \int \frac{d s}{v_{11}}(\ldots), \quad, \quad \tau_{B} \equiv \int \frac{d s}{v_{11}},
$$

$\omega_{0}$ is the magnetic-drift frequency, and $J_{0}$ is the Bessel funcicion introduced to allow for FLR effects. This equation may be put in a variational form by multiplying by $\phi$ and integrating over the field line to obtain

$\sum \frac{q^{2}}{m} \int d \varepsilon d \mu \tau_{B} \frac{\partial f}{\partial \varepsilon}\left\{\overline{(\phi-\bar{\phi})^{2}}+\bar{\phi}^{2}\left[\left(\frac{\omega_{*}-\bar{\omega}_{D}}{\omega-\bar{\omega}_{D}}\right)+k^{2} \rho^{2}\left(\frac{\omega-\bar{\omega}_{D}}{\omega-\bar{\omega}_{D}}\right)\right]\right\}=0$,

where we have introduced the Larmor radius $\rho$ upon expanding $J_{0}^{2}$ and defined

$$
\vec{u}_{\star} \equiv-\frac{\underline{k x b} \cdot \nabla f}{\Omega \partial f / \partial \varepsilon}
$$

Charge neutrality (or its radial derivative) can be expressed in the same notation by

$$
\left.\sum \frac{q^{2}}{m} \int \operatorname{ded\mu } \tau_{B} \frac{\partial f}{\partial \varepsilon} \overline{\left(\phi^{2}\right.}{\hat{u_{k}}}_{k}-\overline{\phi^{2}} \omega_{D}\right)=0 \text {, }
$$

which has been obtained by multiplying the local charge condition by $\phi^{2}$ and integrating over the field line.

The orift frequency $\omega_{D}=\omega_{E}+\omega_{B}$ contains the $\underline{E} \times \underline{B}$ drift,

$$
\omega_{E}=\frac{\mathbf{c}}{B} \underline{k} \underline{\underline{b}} \cdot \nabla \Phi,
$$


and the magnetic drift,

$$
\omega_{B}=\frac{1}{\Omega} \underline{k} \underline{\underline{b}} \cdot\left(\mu \nabla B+v_{\mathbb{1}}^{2} \boldsymbol{k}\right),
$$

where $\underline{\underline{k}} \equiv \underline{\mathrm{b}}$ - F $\underline{\mathrm{b}}$. Equation $\mathbf{0 . 1}$ becomes quite complex when the variation in $\omega_{D}$, which is due primarily to variation in $\omega_{E}$, is comparable to $\omega_{\text {. We do }}$ not consider here such circumstances and assume that $\omega_{0}$ can be written as

$$
\omega_{D}=\omega_{0}+\omega_{B},
$$

where $\omega_{0}$ is the constant, species- and $z$-independent $E \times B$-drift frequency of the central cell. We take $\omega_{B} \ll \omega-\omega_{0}$, and expand the denominators of Eq. D.1. When this is added to Eq. D.2 and divided by $w-\omega_{0}$, with $\omega+\omega+\omega_{0}$ and higher order quantities are discarded, we obtain the result

$$
\begin{gathered}
\sum \frac{g^{2}}{m} \int \operatorname{d\varepsilon d} \mu \tau_{B} \frac{\partial f}{\partial \varepsilon}\left\{\left[\overline{(\phi-\bar{\phi})^{2}}+k^{2} \rho^{2} \bar{\phi}^{2}\right]\left(\omega+\omega_{0}-\hat{\omega}_{\star}\right)\right. \\
\left.+\frac{\bar{\phi}^{2} \frac{\omega_{B}}{\omega}}{\omega}\left(\hat{\omega}_{\star}-\omega_{0}\right)\right\}=0 .
\end{gathered}
$$

In this approximation, $\hat{\omega}_{k}-\omega_{0}$ corresponds to the total density gradient because of the flux dependence of $f$ and of $\Phi$, so that $\hat{\omega}_{\star}-\omega_{0}=\omega_{\star}$ is the familiar diamagnetic drift frequency.

In this form, one can identify the Debye shielding, the polarization, the FLR, and the bounce-averaged curvature-drive terms. The term $(\bar{\phi}-\bar{\phi})^{2}\left(\omega_{\star}-\omega_{0}\right)$ describes a coupling to drift waves brought about by the c, $\mu$, q dependence of particle turning points and, therefore, $\bar{\phi}$, in the combined magnetic and electrostatic fields. This term is absent in the equivalent tokanak calculation.

When the drift and FLR effects are neglected, the variational form becomes an energy principle for $\omega^{2}$, which is a minimum at the eigenfrequency

$$
\omega^{2}=-\frac{\sum \frac{q^{2}}{m} \int \operatorname{ded} \mu \tau_{B} \frac{\partial f}{\partial \varepsilon} \bar{\phi}^{2}\left(\omega_{k}-\omega_{f}\right) \omega_{B}}{\sum \frac{q^{2}}{m} \int \operatorname{ded} \mu \tau_{B} \frac{\partial f}{\partial \varepsilon}\left[(\phi-\bar{\phi})^{2}+k^{2} \rho^{2} \bar{\phi}^{2}\right]} .
$$


Provided trial functions are chosen that vanish in the anchor where $\left(\hat{\omega}_{k}-\omega_{0}\right) \omega_{B}<0$, there is always an instability in this approximation. If the $(\overline{\phi-\phi})^{2}$ factor is small, the growth rate will be the MHO growth rate in the absence of the anchors. This Debye term can be small when very few particles pass between the regions of good and bad curvature. In such circumstances, a perturbation such as that sketched in Fig. D-1 minimizes $\omega^{2}$. In the long central cell, $\phi \approx \bar{\phi}$, so that all particles urapped there do not contribute. For those that do pass between the regions, i.e., in and out of the perturbation,

$$
\tau_{B} \overline{(\phi-\bar{\phi})^{2}}=\int \frac{d s}{u_{\|}}(\phi-\bar{\phi})^{2} \approx \frac{2 L_{a}}{v_{n}} \phi_{c}^{2},
$$

where $L_{a}$ is the length of one anchor and $\phi_{c}$ is the perturbation level in the central cell. If we define the characteristic MHD growth rate of the upfavorable curvature region alone as

$$
\gamma_{C}^{2} \equiv-\frac{\sum \frac{q^{2}}{m} \int \operatorname{ded\mu } \tau_{B} \frac{\partial f}{\partial \varepsilon} \bar{\omega}_{B}\left(\hat{\omega}_{k}-\psi_{0}\right)}{\sum \frac{q^{2}}{m} \int \operatorname{ded\mu } \tau_{B} k^{2} \rho^{2} \frac{\partial f}{\partial \varepsilon}},
$$

we find

$$
\omega^{2}=\frac{\gamma_{c}^{2}}{1+\Sigma \frac{T}{k^{2} \rho^{2}} \frac{B_{c}}{n_{c} L_{c}} \int_{\phi=0} \frac{d s}{B} \hat{n}},
$$

where $\hat{n}$ is the density of particles passing into and out of the perturbation, and the integration is carried out over the region that "'e perturbation vanishes. According to how many ions are trapped in t. ansition region, in addition to those passing directly from the central cell, the eigenfunction will minimize this integral of $\hat{n}$. When both the positive potential peak and thermal barrier are placed in the axicell, as is possible in one operating mode of the axicell configuration, $\hat{n} / n_{c}$ is very small. The special susceptibility of this operating mode to ES ballooning was first pointed out by Berk and Rosenbluth ${ }^{D-1}$. 

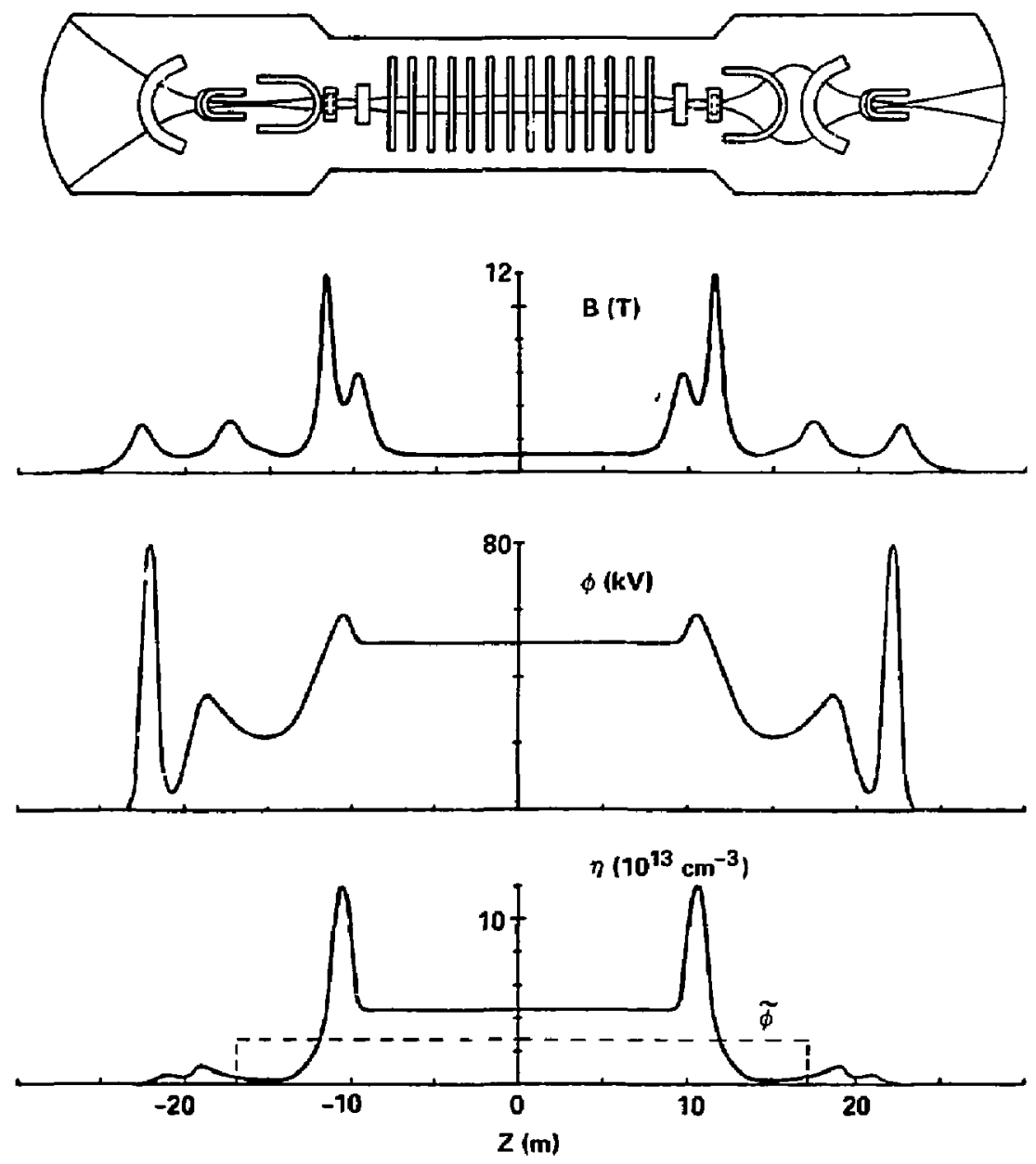

Fig. D-1. Profiles of field $B$, density $n$, and potential $\phi$ for the MFTF-B Axicell design. The dashed line represents the worst ES ballooning mode, which occurs when the density in the transition is very low. 
The drift term in Eq. D.1, neglected in obtaining Eq. D.6, can play an important role in stabilizing the basic mode described by Eq. 0.6 by coupling it to a drift wave ${ }^{D-2, D-3}$. The process is very similiar to familiar FLR stabilization of curvature-driven modes and is described by including $\omega_{\star}-\omega_{0}$ in Eq. D.3. As may be observed in Eq. D.3, particles trapped in a region of constant $\phi$ have no drift contribution, just as they have no Debye contribution. The FLR term, of course, contributes everywere.

A second point to be recognized from Eq. $D .3$ is that the ion-drift term has the same sign as the ion-FLR term and is, therefore, additive. The electron-drift term has the opposite sign. The magnitude of each term is weighted by $(\overline{\phi-\bar{\phi}})^{2}$, and this term is in turn increased by the "overshoot", or the distance of reflection of a particle beyond the place where $\phi$ gues to zero. Thus, configurations are preferred in which ions carry further beyond the perturbation than the electrons, and the axicell configuration is of this type.

The variational form of Eq. D.3 can be used to compare the axicell and A-ce]l geometries with regard to stability to the ES ballooning mode. Consider first the axicell design shown in Fig. D-2. As a trial function, take $\phi=1$ through the central cell, $\phi=1-\alpha$ in the transition, and $\phi=0$ in the anchor or yin yang. The parameter $\alpha$ will be varied to achieve a minimum in the stabilizing contribution. Changes in $\phi$ are located at the high mirror peak of the axicell and the inside edge of the thermal barrier (see dashed line in Fig. 0-2).

The ion distribution in the transition is composed of a portion that streams directly from the central cell (to be confined by the positive potential of the anchor, $n_{\text {pass }}$ ) and a population of ions that have become trapped between this potential and the high-field coil of the axcicell. With good pumping in the transition region, the distribution of these trapped ions is close to that of the passing component and, therefore, is beam-like in character. We denote their relative density by $g_{b}-I\left(g_{b}\right.$ is the total density in the transition normalized to the passing component; the two spatial profiles throughout the transition and anchor regions are nearly equal). We then compute different ayerages for different classes of ions. Introducing the bounce time in each region--central cell, transition, and anchor $\left(\tau_{c}, \tau_{t}\right.$, $\tau_{a}$, respectively)--we have for those ions trapped in the central cell, 

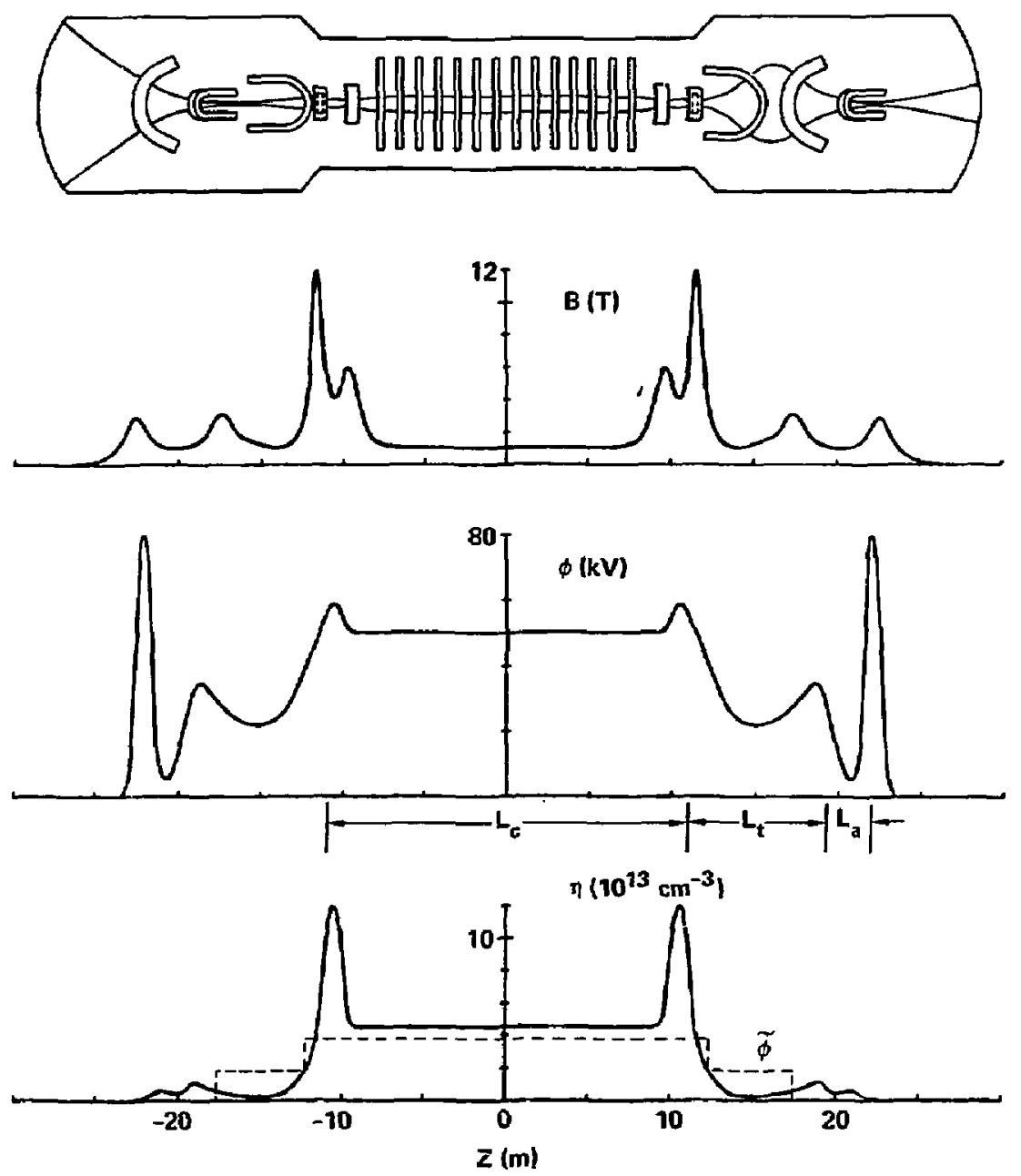

Fig. D-2. Field, density, and potential profiles for the MFTF-e Axicell design. The dashed line represents the trial function, having variable transition-region value, when the plasma density in the transition is low but significant. 


$$
\bar{\phi}=1 ;
$$

for those untrapped in the central cell and confined by the anchor potential,

$$
\bar{\phi}=\frac{\tau_{c}+2\left(\tau_{t}+\tau_{a}\right)(1-a)}{\tau_{c}+2\left(\tau_{t}+\tau_{a}\right)} \underset{\tau_{c} \gg \tau_{t}, \tau_{a}}{\longrightarrow} ;
$$

and for those trapped in the transition-anchor region,

$$
i=(1-a) \frac{\tau_{t}}{\tau_{t}+\tau_{a}} \text {. }
$$

Here $\tau_{c}, \tau_{t}, \tau_{a}$ are proportional to the lengths shown in Fig. 0-2.

Although the ion distribution in the transition region is of a streaming type, the electrois that neutralize these ions wiil be locally Maxwellian and very few of them will pass through the high-field axicell coil. Thus, to a good approximation, the electrons in each cell are confined to that cell, their * $\bar{p}$ in each cell, and they drop out of the shielding and drift terms of 2. 1.7 .

To caiculate the effect of the beam-1ike ion distribution in the transition regions, we temporarily restore the definition of $\omega_{*}$ and rewrite Eq. D.3 as

$$
\begin{aligned}
& \sum \frac{q^{2}}{m} \int d \varepsilon d \mu \tau_{B}\left[\overline{(\phi-\bar{\phi})^{2}}+k^{2} \rho^{2} \bar{\phi}^{2}\right]\left[\left(\omega+\omega_{0}\right) \frac{\partial f}{\partial E}+\frac{1}{\Omega} \underline{k} \underline{b} \underline{b} \cdot \nabla f\right] \\
& -\frac{\bar{\phi}^{2} \bar{\omega}_{B}}{\omega}\left[\omega_{0} \frac{\partial f}{\partial \varepsilon}+\frac{1}{\lambda} \underline{k} \underline{b} \cdot \nabla f\right] \mid=0 \text {. }
\end{aligned}
$$

Because $\partial f / \partial \varepsilon>0$ for trapped ions, such terms must be treated with same care. As implied above, we treat $\Phi$ as independent of $\varepsilon, \mu$, except for the differences arising from the trapped and untrapped orbits. Thus, for the term not containing af/Je, we obtain 


$$
\begin{aligned}
& \int \mathrm{d} \varepsilon \mathrm{d} \mu \tau_{B} \overline{(\phi-\bar{\phi})^{2}} \frac{1}{\Omega} \underline{k \times b} \cdot \nabla f \\
& =\frac{2 \hat{\omega}_{\star}}{T_{c}}\left[\frac{\alpha^{2} \tau_{t}+\tau_{a}}{\tau_{t}+\tau_{a}}+\left(g_{b}-1\right)(1-\alpha)^{2} \frac{\tau_{t} \tau_{a}}{\left(\tau_{t}+\tau_{a}\right)^{2}}\right] \int_{t+a} \frac{d \ell}{B} n_{p a s s},
\end{aligned}
$$

where $T_{c}$ is the central-cell ion temperature, and the passing density $n_{\text {pass }}$ is integrated over the transition and anchor regions. In the $\partial f / \partial \varepsilon$ term, we integrate in energy, expressing the result in terms of the ccrmon value of $f$ at the separatrix and $\Delta(\overline{\phi-\phi})^{2}$, which is the jump in $(\overline{\phi-\phi})^{2}$ between passing and trapped icns. Estimating

$$
\int d \mu \tau_{B} f \mid \text { separatrix } \approx \frac{1}{T_{c}} 2 \int_{t+a} \frac{d \ell}{B} n_{\text {pass }},
$$

we obtain

$$
\begin{aligned}
\int d \mu \tau_{B} f & {\overline{\Delta(\phi-\bar{\phi})^{2}}}_{\text {separatrix }} \\
& \approx-\frac{1}{T_{c}}\left[\frac{a^{2} \tau_{t}+\tau_{a}}{\tau_{t}+\tau_{a}}-(1-\alpha)^{2} \frac{\tau_{t} \tau_{a}}{\left(\tau_{t}+\tau_{a}\right)^{2}}\right] \int \frac{d \ell}{B} n_{p a s s} .
\end{aligned}
$$

Integrals containing $\bar{\phi}^{2}$ have their principal contributions from the centra? cell when these considerations do not apply because of the nearly Maxweilian nature of the distributions there.

Combining these results, we cbtain from Eq. $D .3$ and the assumed perturbation (negiecting the FLR term so as to model a rigid perturbatian), 


$$
\begin{aligned}
\omega(\omega= & \left.\omega_{0}\right)\left[\frac{\alpha^{2} \tau_{t}+\tau_{a}}{\tau_{t}+\tau_{a}}-(1-\alpha)^{2} \frac{\tau_{t} \tau_{a}}{\left(\tau_{t}+\tau_{a}\right)^{2}}\right] 2 \int_{t+a} \frac{d l}{B} n_{p a s s} \\
& -\omega \hat{w}_{\star}\left[\frac{a^{2} \tau_{t}+\tau_{a}}{\tau_{t}+\tau_{a}}+\left(g_{b}-1\right)(1-\alpha)^{2} \frac{\tau_{t} \tau_{a}}{\left(\tau_{t}+\tau_{a}\right)^{2}}\right] 2 \int_{t+a} \frac{d \ell}{B} n_{p a s s} \\
& +\left[\omega^{2} k^{2} p^{2}+\bar{w}_{B}\left(\omega_{\star}-\omega_{0}\right)\right] \int_{c} \frac{d l}{B} n_{c}=0,
\end{aligned}
$$

where the subscript $c$ denotes the central cell.

The choice of a to yield the most unstable mode, in general. involves the parameters $\hat{\omega}_{*} / \omega_{0}$ and $\hat{\omega}_{k} / \bar{\omega}_{B}$. Here we set $\alpha=1$, thereby eliminating the stabilizing effects of positive $\partial f / \partial \varepsilon$ and $g_{b} \neq 1$ in the transition region. This perturbation has $\phi=0$ in the transition region and anchor. Solutions are stable provided

$$
\frac{1}{4} \bar{\omega}_{B}\left(\hat{\omega}_{k}-\omega_{0}\right) \int_{c} \frac{d l}{B} n_{c}<\frac{\left(\hat{\omega}_{*}-\omega_{0}\right)^{2}\left(2 \int_{t+a} \frac{d l}{B} n_{p}\right)^{2}}{\left(k^{2} \rho^{2} \int_{c} \frac{d \ell}{B} n_{c}+2 \int_{t+a} \frac{d l}{B} n_{p}\right)} .
$$

In terms of the growth rate $\gamma_{c}$ introduced in Eq. 0.5 , this becomes

$$
\frac{\omega_{k}^{2}}{\gamma_{c}^{2}}>\frac{4 A^{2}}{(1+A)} \text {. }
$$

where

$$
A=\frac{1}{k^{2} \rho^{2}} \frac{2 \int_{t+a} \frac{d l}{B} n_{p}}{\int_{c} \frac{d l}{B} n_{c}}
$$

and we have introduced the familiar $\omega_{*}$.

In the A-cell geometry shown in Fig. D-3, we assume that the worst mode is one that vanishes in the $y$ in yang and the A-cell. All but a fraction of electrons are free to pass through the anchor, and only these see an average potential 


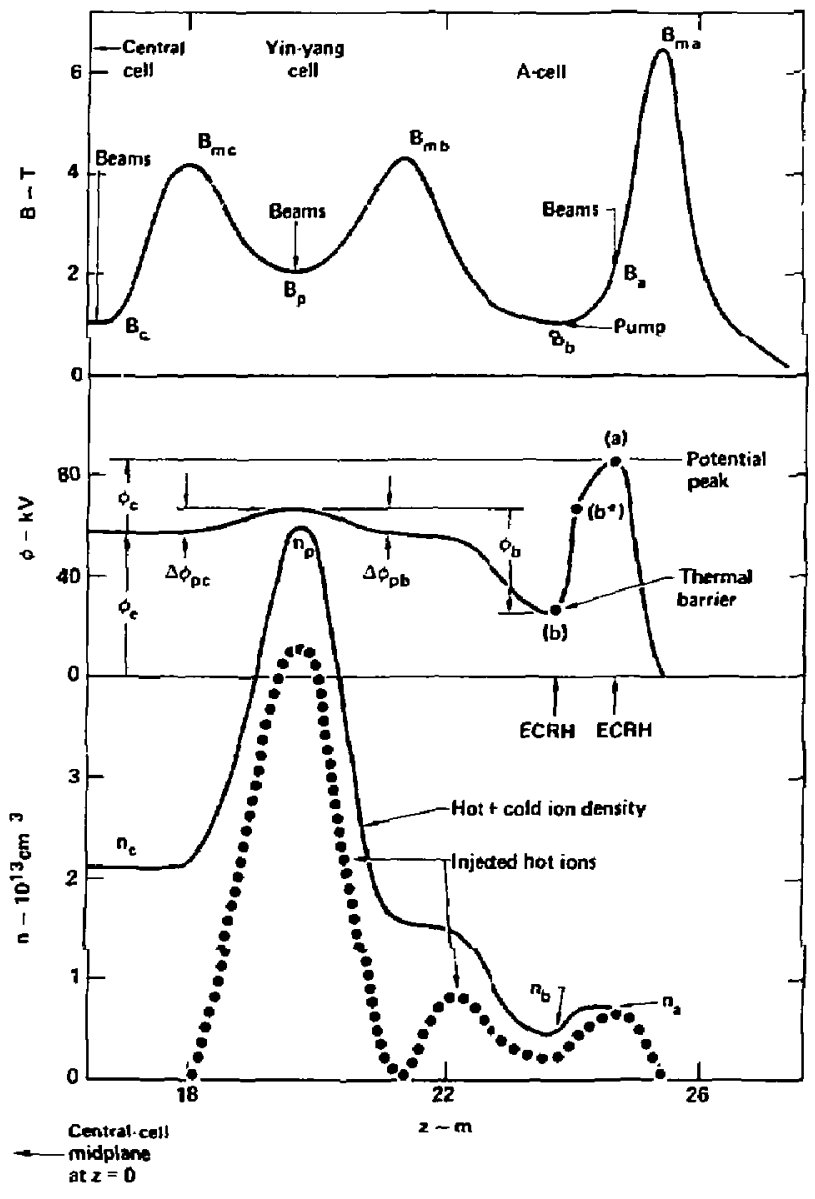

Fig. D-3. Profiles of field, potential, and density at one end of MFTF-B when operated with thermal barriers of the A-celi type. The dashed line represents the assumed worst perturbation that excludes the favorable yin-yang curvature. 


$$
\bar{\phi}_{e}=\frac{\tau_{c}}{\tau_{c}+2\left(\tau_{A}+\tau_{a}\right)},
$$

where $A$ refers to $A$-cell and, as before, a to the anchor, or yin-yang.

If there is no local potential rise $\Delta \varphi_{p}$ in the anchor, the same fraction of ions pass through as electrons and there is no drift term at all. In the presence of $\Delta \varphi_{p}$, a drift term develops. However, because it is generated by electrons, this term has the opposite sign as the familiar ion FLR term.

Neglecting the $\varepsilon$, $u$ dependence of the various transit times, we arrive at the following estimates,

$$
\begin{aligned}
& \left\langle\tau_{B} \overline{(\phi-\bar{\phi})^{2}}\right\rangle_{\text {ions }}=\frac{1}{R_{C}} e^{-\Delta \varphi_{p} / T_{i c}} \frac{2\left(L_{a}+L_{A}\right)}{L_{C}} \\
& \left\langle\tau_{B} \overline{(\phi-\Phi)^{2}}\right\rangle_{\text {e lectrons }}=\frac{1}{R_{C}} \frac{2\left(L_{a}+L_{A}\right)}{L_{C}}
\end{aligned}
$$

where $\ldots$ denotes the $\varepsilon, \mu$ average, and $R_{c}$ is the mirror ratio seen by the central cell. When allowance is made for $\omega_{k_{e}}=-\omega_{k_{j}} T_{e} / T_{j}$, these results lead to the dispersion relation

$$
\left(1+\frac{A_{1}}{m^{2}}\right) \omega^{2}-\omega_{*_{i}}\left(m-1+\frac{A_{2}}{m}\right) \omega+\gamma_{c}^{2}=0,
$$

where

$$
\begin{aligned}
& A_{1}=\frac{1}{R_{c}}\left(\frac{T_{i c}}{T_{e c}}+e^{-\Delta \phi_{p} / T_{i c}}\right) \frac{r_{c}^{2}}{\rho_{i}^{2}} \frac{2\left(L_{a}+L_{A}\right)}{L_{c}} . \\
& A_{2}=-\frac{1}{R_{c}}\left(1-e^{-\Delta \phi_{p} / T_{i c}}\right) \frac{r_{c}^{2}}{\rho_{i}^{2}} \frac{2\left(L_{a}+L_{A}\right)}{L_{c}} .
\end{aligned}
$$

Evaluations of the A-factors for the two magnet sets are given in Sec. I. Comparisons of the two geometries shows the A-values for the A-cell configuration are larger. However, the sign of the drift term is such as to cancel the FLR term, giving instability at finite m. 


\section{REFERENCES}

D-1. H.L. Berk and M.N. Rosenbluth, LLHL, private communication.

D-2. H.L. Berk, M.N. Rosenbluth, H.V. Wong, T. Antonsen, and D.E. Baldwin, Fast-Growing Trapped-Particle Modes in Tandem Mirrors, Institute for Fusion Studies, University of Texas, Austin, TX, Report IFSR-59 (1982).

D-3. L.D. Pearlstein, D.B. Baldwin, R.H. Cohen, T.K. Fowler, and B.G. Logan, "Stabilization of Tandem-Mirror Trapped-Particle Modes by Incomplete Cancellation of Trapped-Particle Drifts," presented at 1982 Sherwood Meeting, Annual Controlled Fusion Theory Conference, Santa Fe, NM, Apri 1 25-28, 1982. 


\section{APPENDIX E}

\section{SLOSHING IONS IN THE MFTF-B ANCHOR}

M. E. Rensink

\section{INTROOUCTION}

In both the A-cell and axicell configurations for MFTF-B, sloshing ions play a key role in microstability considerations and in creation of the potential that plugs the solenoid. In the A-cell configuration the sloshing-ion distribution and plugging potential were produced in the A-cell itself. For the new axicell configuration, the sloshing-ion distribution and plugging potential are produced in the anchor (yin yang). Here, we report on some bounce-average Fokker-Planck. calculations for sloshing ions in the MFTF-B anchor. As in the previous designs, sloshing ions are formed by off-midplane neutral-beam injection perpendicular to the magnetic field.

\section{MAGNETIC FIELD}

The axial magnetic field profile for the stretched yin-yang configuration is shown in fig. E-1. The vacuum magnetic field, $B_{v a c}(z)$, is obtained from an EFFI code run. The finite-beta depression of the vacuum magnetic field is estimated by means of the long-thin approximation,

$$
B(z)=B_{v a c}(z) \sqrt{T-B(z)}
$$

where our model for $B(z)$ is

$$
B(z)=B(0)\left(\frac{B_{M}-B_{v a c}(z)}{B_{M}-B_{v a c}(0)}\right) \text {. }
$$

We assume a midplane $(z=0)$ beta value $\beta(0)$ of 0.55 , so the vacuum mirror ratio of 2.91 increases to 4.34 , mainly because of the hot magnetically-confined electrons. 


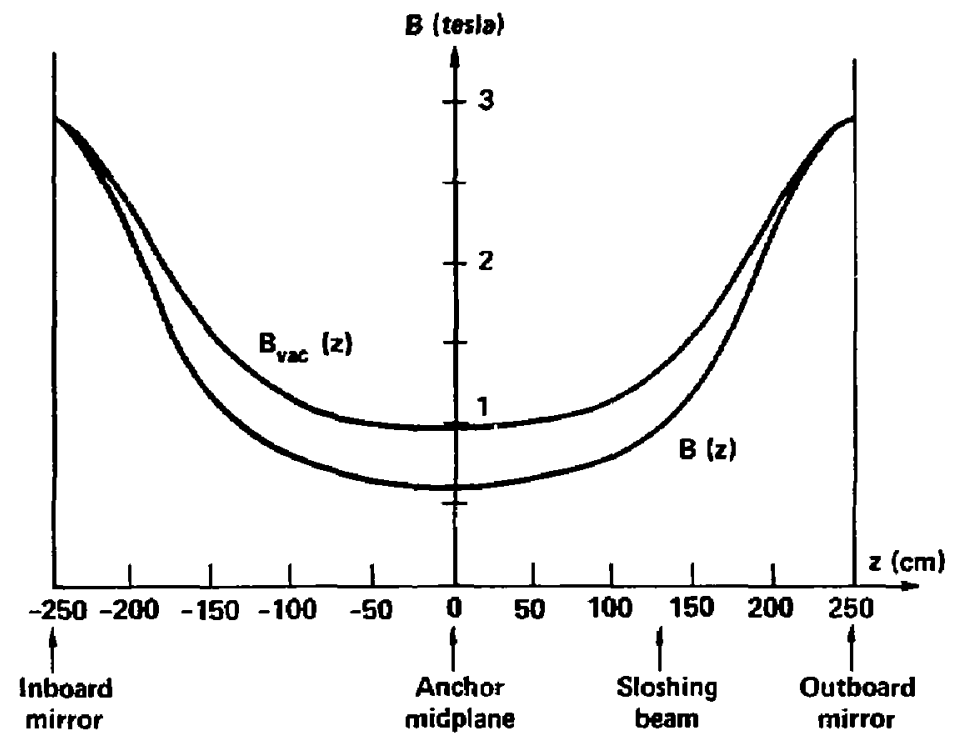

Fig. E-1. Axial magnetic-field profile in anchor. 


\section{3. electrostatic potential}

The axial potential profile, $\Phi(z)$, is sketched in Fig. E-2. This potential is derived by setting the local charge density to zero, including contributions from cold, warm, and hot electrons as well as passing, trapped, and sloshing ions. The self-consistent calculation of this potential is a formidable problem, so for the sloshing-ion calculations described here we assume a fixed form for the potential as part of our input data.

on the outboard side of the anchor midplane, we assume a piece-wise quadratic form for $\Phi[\psi]$, where $\psi \equiv B(z) / B(0)$ is the local mirror ratio;

$$
\begin{aligned}
\Phi[\psi] & =\Phi_{a}-\left(\Phi_{a}-\Phi_{0}\right)\left(\frac{\psi_{a}-\psi}{\psi_{a}-1}\right)^{2}, 1 \leq \psi \leq \psi_{a} ; \\
& =\Phi_{a}-\left(\Phi_{a}-\Phi_{m}\right)\left(\frac{\psi-\psi_{a}}{\psi_{m}-\psi_{a}}\right)^{2}, \quad \psi_{a} \leq \psi \leq \psi_{m} .
\end{aligned}
$$

The parameters used in our simulations were:

$$
\begin{array}{ll}
\Phi_{0}=20.0 \mathrm{keV} ; & \\
\Phi_{\mathrm{a}}=80.0 \mathrm{keV}, & \psi_{\mathrm{a}}=1.50 ; \\
\Phi_{\mathrm{m}}=0.0 \mathrm{keV}, & \psi_{\mathrm{m}}=4.34 .
\end{array}
$$

On the inboard side of the anchor midplane we assume a net mirror ratio $R_{L}=4.34$ and a potential rise (midplane to inboard mirror) $\Delta \Phi_{L}$ of $32.5 \mathrm{keV}$. These parameters are important in mapping the passing- (solenoid-) ion distribution from the inboard mirror to the midplane, which is the reftrence point for the bounce-average Fokker-Planck code. The detailed profile of $\Phi$ on the inboard side does not enter the calculations because in performing orbit averages, the code assumes symmetry with respect to the midplane.

\section{NEUTRAL BEAM INJECTION}

Neutral-beam injection is represented by various source and loss terms in the Fokker-Planck equation for the ions. These may be written schematically in the form 


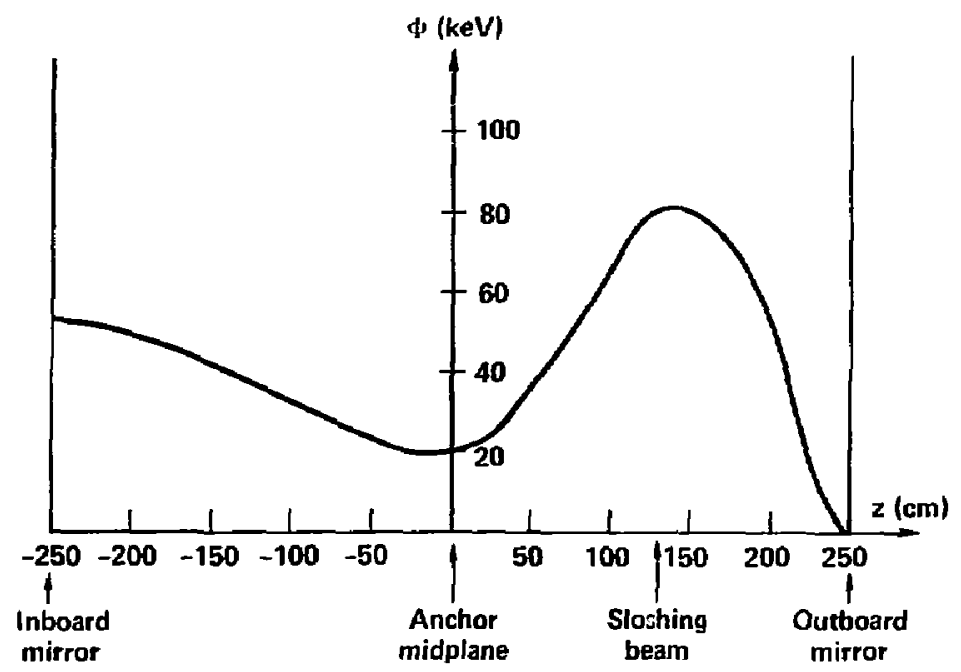

Fig. E-2. Potential profile in an-hor. 


$$
\begin{aligned}
\frac{\partial}{\partial t} f_{i}(z, v, \theta)=c\left(f_{i}\right)+\sum_{b} \alpha_{b}(z) & {\left[\left(v_{I}^{b}+v_{c x}^{b}\right) n_{i}(z) S_{b}(v, \theta)\right.} \\
& \left.-v_{c x}^{b} f_{j}(z, v, \theta)\right] .
\end{aligned}
$$

The first term, $c\left(f_{j}\right)$, is the Fokker-Planck collision term. The remaining terms represent ionization and charge exchange for each beam in the problem. The factor $a_{b}(z)$ defines the spatial profile of the incident neutral beam. Typicaliy, we assume the Gaussian form

$$
a_{b}(z)=\exp \left[-\left(z-z_{b}\right)^{2} /\left(\Delta z_{b}\right)^{2}\right] \text {. }
$$

where $z_{b}$ is the firjectron porit and $\Delta z_{b}$ is the axidi extent of the bean. For sloshing beams in the MFTF-B anchor, we inject at the position of the potent: peak, $z_{b}=130 \mathrm{~cm}$, as indicated in Figs. E-1 and E-2. For we11-collimated beams focused at the magnetic axis the spatial extent is $\Delta z_{b}= \pm 10 \mathrm{~cm}$ for perpendicular injection.

The local source strength (particles $/ \mathrm{cm}^{3} / \mathrm{s}$ ) at the injection fuint is proportional to the local ion density for ionization and charge-exchange processes. The rate coefficients $v_{I}^{b}$ and $v_{c x}^{b}$ are specified as input parameters. These are related to incident neutral-beam currents and ionization and charge-exchange cross sections, e.g.,

$$
\begin{aligned}
& v_{I}^{b}=n_{b}(\overline{\sigma v})_{I}^{b}, \\
& n_{d}=J_{d} / e y_{b} .
\end{aligned}
$$

where $J_{b}$ is the incident neutral-beam current density, $v_{b}$ is the beam velocity, and $(\overline{\sigma \sigma})_{I}^{b}$ is the impact-ionization rate parameter (from both el cctrons and ions).

The velocity-space shape factor, $S_{b}(v, \theta)$, describes the energy and orientation of the neutral beam and has the form

$$
s_{b}(v, \theta)=k_{b} \exp \left[-\lambda_{b}\left(v-v_{b}\right)^{2}-y_{b}\left(\cos \theta-\cos \theta_{b}\right)^{2-},\right.
$$

where $\left(v_{b}, \theta_{b}\right)$ specify the mean velocity and pitch angle and $\left(\lambda_{b}, \gamma_{b}\right)$ 
define the spread in these quantities. The normalization constant, $k_{b}$, is defined such that

$$
\int d^{3}, s_{b}(v, \theta)=1
$$

\section{BEAM SPECIES MIX}

There are three energetic components for the injected neutral beams. Most of the incident neutrals have the $f u 1180-\mathrm{keV}$ energy, but there are Smaller fractions with half-energy $(40 \mathrm{keV})$ and third-energy $(27 \mathrm{kev})$. For our sloshing-ion beams, we have assumed a 90/5/5 molecular-source-current mix, which leads to incident atomic currents at the plasma in the ratio $83 / 8 / 9$ when beam divergences are taken into account. The half-and third-energy beam components tend to reduce the mean energy of the trapped ions because they constitute a finite fraction of the total incident beam. This effect is accentuated because the charge-exchange cross section at $27 \mathrm{keV}$ and $40 \mathrm{keV}$ is larger than at $80 \mathrm{keV}$, leading to a iarger trapping fraction for the half - and third-energy components. Also, if the half- and third-energy ions from the sloshing beam are not trapped, then charge exchange between these components and the sloshing ions acts as a loss mechanism for the ions and leads to increased sloshing beam current requirements.

\section{HIGH-ENERGY PUMP BEAM (HEPB)}

The high-energy pump beam is directed along the magnetic axis so that any charge-exchange of this bean with the sloshing or trapped ions produces an untrapped ion. Thus, the HEPB removes inns from the anchor. The rate parameter $(\overline{\sigma v})_{C X}^{H E P B}$ can vary significantiy with axial position because the charge-exchange cross section is a strong function of the relative velocity between the axially directed HEPB and the co- or counter-streaming components of the sloshing ions. This effect is included in the specification of the profile factor $\alpha_{b}(z)$ for the HEPB. For the MFTF-B anchor, $n_{H E P B}(z)$ is approximately uniform for the axially-directed $H E P B$, and the spatial variation of $(\overline{\sigma V})_{C X}^{H E P B}$ is modelled by

$$
a_{H E P B}(z)=\exp \left[-z^{2} / L_{c x}^{2}\right],
$$


where $L_{C X} \approx 250 \mathrm{~cm}$ fits the estimated fall off in $(\sigma)_{C X}^{\text {HEPB }}$ as one moves out from the midplane.

7. LOSS-CONE BOUNDARIES AND PASSING IONS

The velocity-space loss boundary at the midplane of the anchor consists of several segments, as shown in Fig. E-3. For ions to escape through the outboard mirror from the midplane, they must first be able to pass over the pntential peak $\Phi_{a}$ at $\psi_{a}$. Ions to the right of curve A satisfy this condition. If the magnetic moment of these ions is not too large, they will be able to pass through the outboard-mirror throat and escape from the plasma. Ions to the $r$ ight of both curves $A$ and $M$ satisfy these conditions. It is also

'ble for ions to escape through the inboard mirror and join the passing iuns in the solenoid or in the transition region between the axicell and potential peak at $\psi_{a}$ in the anchor. Ions in the shaded region to the right of curve $L$ but to the left of curve $A$ satisfy this csindition, and we call these the "passing" ions.

We assume that the passing ions have a Maxwellian distribution characterized by the solenoid-ion temperature $\left(T_{i c}=15 \mathrm{keV}\right.$ for nominal MFTF-B operation). The ciensity of the passing jons depends on the strength of the charge-exchange pumping in the transition region. In this report we present results for several plausible values of the passing-ion density.

\section{RESULTS}

We present results for three different values of the passing-ion density at the inboard-mirror throat, i.e., $n_{M}=2 \times 10^{12}, 4 \times 10^{12}$, and $8 \times 10^{12} \mathrm{~cm}^{-3}$. The incident sloshing beam is held fixed, and in each case the HEPB has been adjusted in intensity to maintain a midplane ion density $n_{0} \approx 3.2 \times 10^{12} \mathrm{~cm}^{-3}$. Table E-1 surmarizes input parameters common to all three cases. Results are sumarized in Table E-2 and in Figs. E-4 to E-7. It should be noted that the density profiles in Fig. E-4 apply only to the outboard side of the anchor.

From Table E-2 we see that the ratio of warm (passing and trapped) ions to sloshing ions is largest for case $C\left(n_{M}=8 \times 10^{12} \mathrm{~cm}^{-3}\right)$ so we expect this to be the most favorable case for microstability. An examination of the 


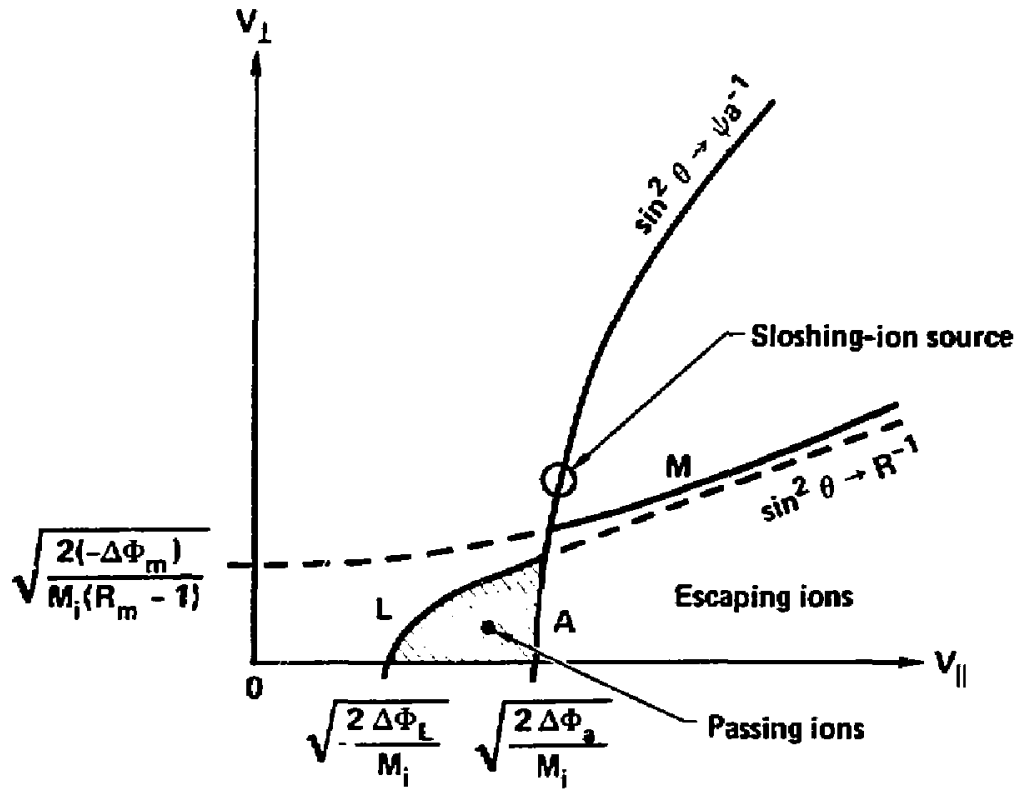

Fig. E-3. Loss-cone boundaries in midplane velocity space of anchor. 
Table E-1. Input parameters for sloshing-ion anchor.

\section{Magnetic Field}

$\begin{array}{ll}\text { Outboard-mirror ratio (net) } & R_{M}=4.34 \\ \text { Inboard-mirror ratic (net) } & R_{L}=4.34 \\ \text { Midplane-to-mirror axial distance (symmetric) } & Z_{m}=248 \mathrm{~cm}\end{array}$

\section{Potential}

Potential drop (midplane to outboard mirror)

$\Delta \phi_{\mathrm{m}}=-20 \mathrm{keV}$

Potential rise (midplane to peak of plug)

$\Delta \Phi_{a}=+60 \mathrm{keV}$

Mirror ratio to peak of plug potential

$\psi_{\mathrm{a}}=1.5$

Potential rise (midplane to inboard mirror)

$\Delta \Phi_{L}=+32.5 \mathrm{keV}$

\section{0-keV Sloshing Beams}

Mirror ratio (midplane to injection point)

Axial distance (midplane to injection point)

$$
\begin{aligned}
& \psi_{i H_{J}}=1.5 \\
& z_{i n j}=130 \mathrm{~cm} \\
& \Delta_{i n j}= \pm 10 \mathrm{~cm} \\
& \theta_{i n j}=90^{\circ} \\
& E_{i n j}^{(0)}=140 \mathrm{keV} \\
& \theta_{i n j}^{(0)}=38^{\circ} \\
& v_{I}^{S L}=13.75 / 3.62 / 7.07 \\
& v_{C x}^{S L}=5.34 / 2.27 / 5.25
\end{aligned}
$$$$
\text { Axial extent of beam }
$$$$
\text { Loca? injection angle }
$$

Equivalent midplane injection energy

Equivalent midplane injection angle

Rate coefficients for full/half/third-energy beam components

Incident sloshing-beam current density (at

injection point on each side of midplane)

$$
\mathrm{j}_{\text {inc ident }}^{S L}=10.4 \mathrm{~mA} / \mathrm{cm}^{2}
$$

\section{0-keV HEPB}
e -folding length for fall off of $(o r)_{C X}^{\text {HEPB }}$
$L_{c x}=248 \mathrm{~cm}$ 
Table E-2. Effect of passing-ion density on anchor parameters.

\begin{tabular}{|c|c|c|c|c|}
\hline & \multicolumn{4}{|c|}{ Case number } \\
\hline & A & B & $\mathbf{C}$ & D \\
\hline Fokker-Planck run & MF25N & MF25K & MF25M & MF250 \\
\hline $\begin{array}{l}\text { Passing-ion density at inboard-mirror } \\
\text { throat, } n_{m}\left(\mathrm{~cm}^{-3}\right)\left(10^{12}\right)\end{array}$ & 2 & 4 & 8 & 0 \\
\hline $\begin{array}{l}\text { HEPB-rate coefficient needed to maintain } \\
\text { to constant midplane density, } \\
v_{\mathrm{CX}}^{\mathrm{HEPB}}\left(\mathrm{s}^{-1}\right)\end{array}$ & 1.50 & 1.83 & 3.30 & 1.83 \\
\hline $\begin{array}{l}\text { Incident HEPB current density; } \\
\text { JEPB } \\
\text { incident }\left(m A / \mathrm{cm}^{2}\right)\end{array}$ & 1.03 & 1.26 & 2.26 & 1.26 \\
\hline Total midplane-ion density, $n\left(\mathrm{~cm}^{-3}\right)\left(10^{12}\right)$ & 3.22 & 3.33 & 3.12 & 2.08 \\
\hline Pasting warm-ion density, $n_{p}\left(\mathrm{~cm}^{-3}\left(10^{12}\right)\right.$ & 0.10 & 0.30 & 0.40 & 0 \\
\hline Trapped warm-ion density, ${ }_{T}\left(\mathrm{~cm}^{-3}\right)\left(10^{12}\right)$ & 0.77 & 1.52 & 2.25 & 0 \\
\hline Sloshing-ion density, $n_{S L}\left(\mathrm{~cm}^{-3}\right)\left(10^{12}\right)$ & 2.35 & 1.61 & 0.47 & 2.08 \\
\hline
\end{tabular}




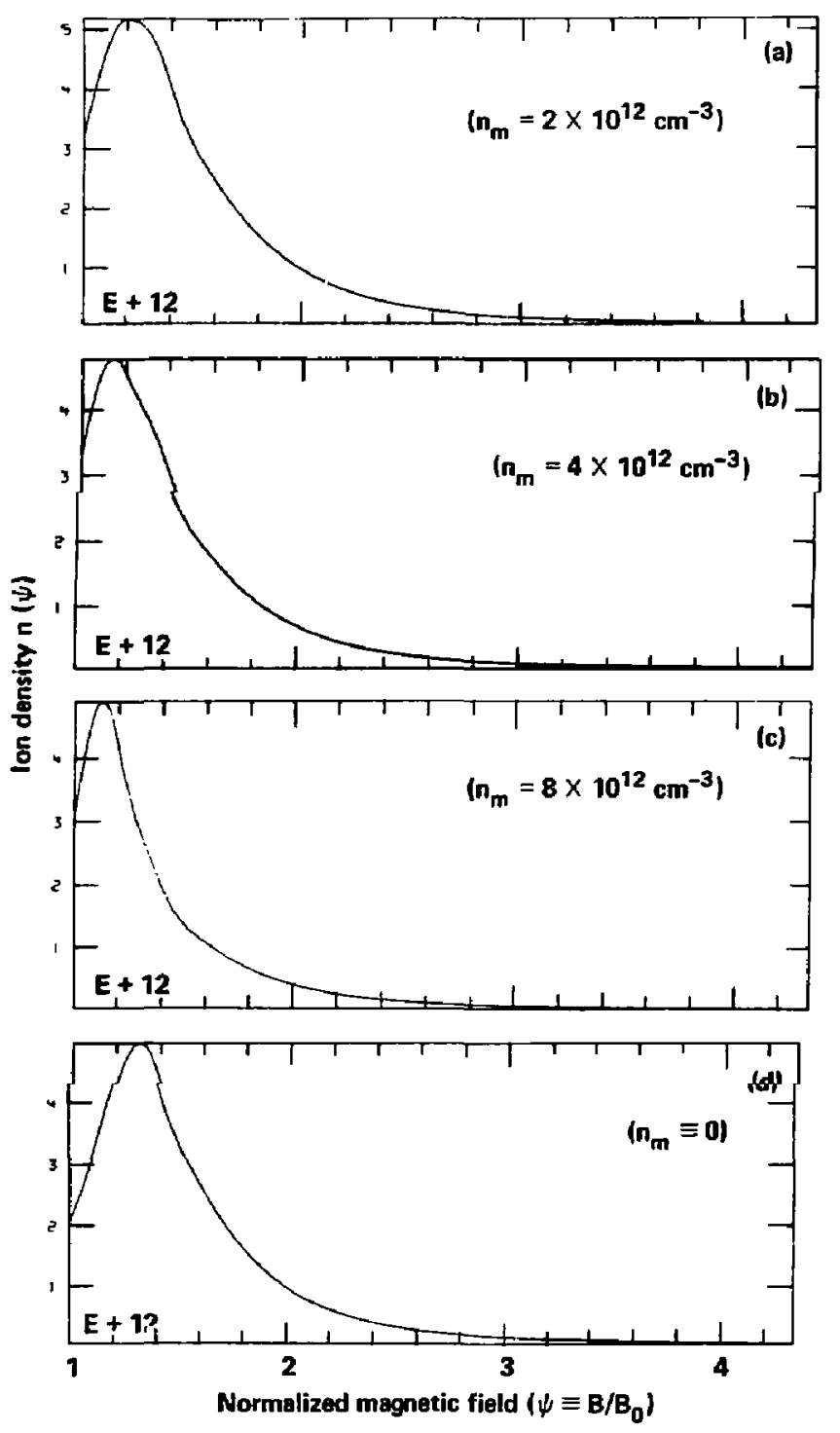

Fig. E-4. Ion density as a function of magnetic field for four values of $n_{m^{*}}$. 

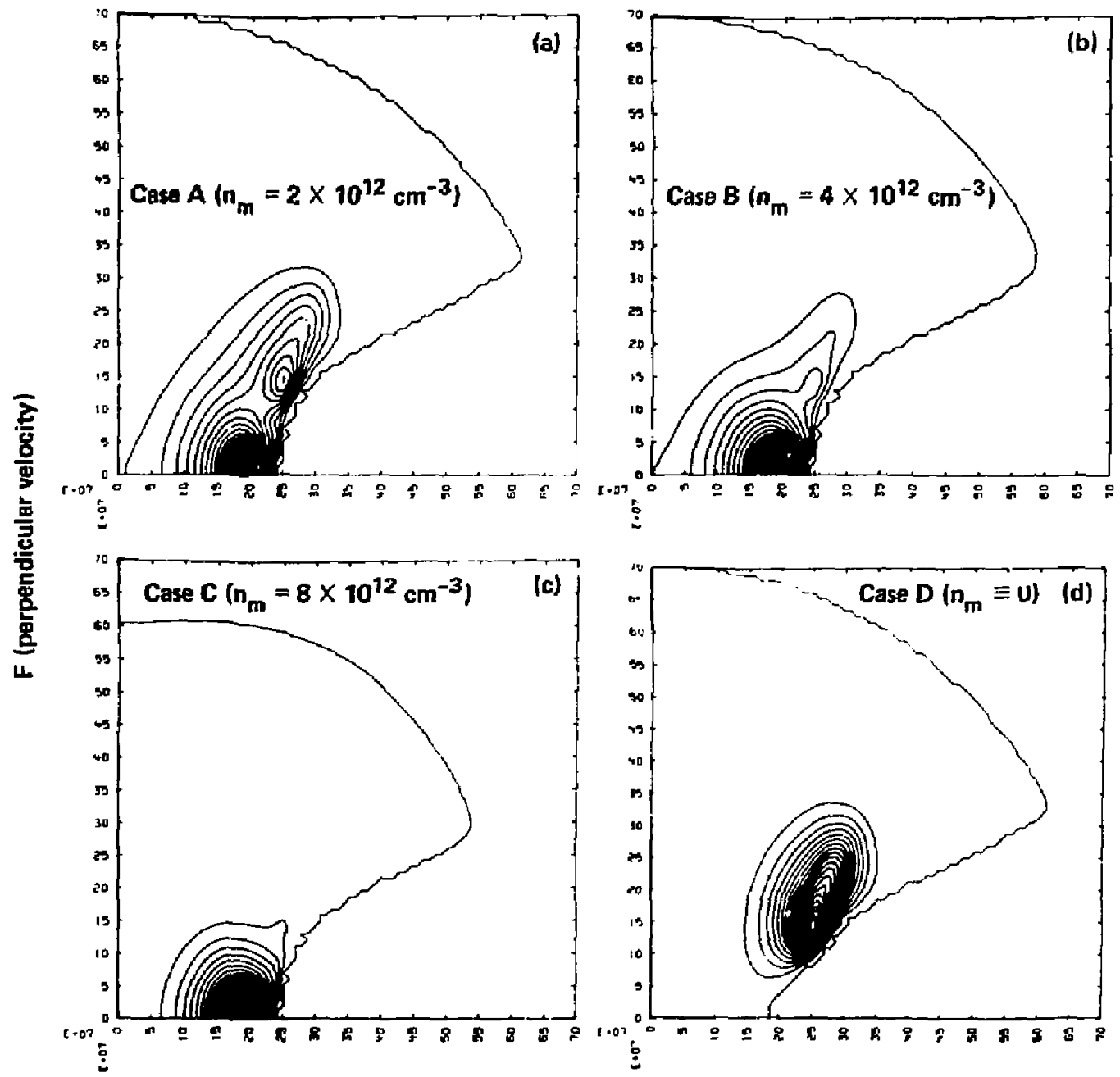

F (parallel velocity)

Fig. E-5. Contour plots of ion-distribution function, $f_{0}\left(v_{1}, v_{11}\right)$, at anchor midplane for four values of $n_{m}$. 

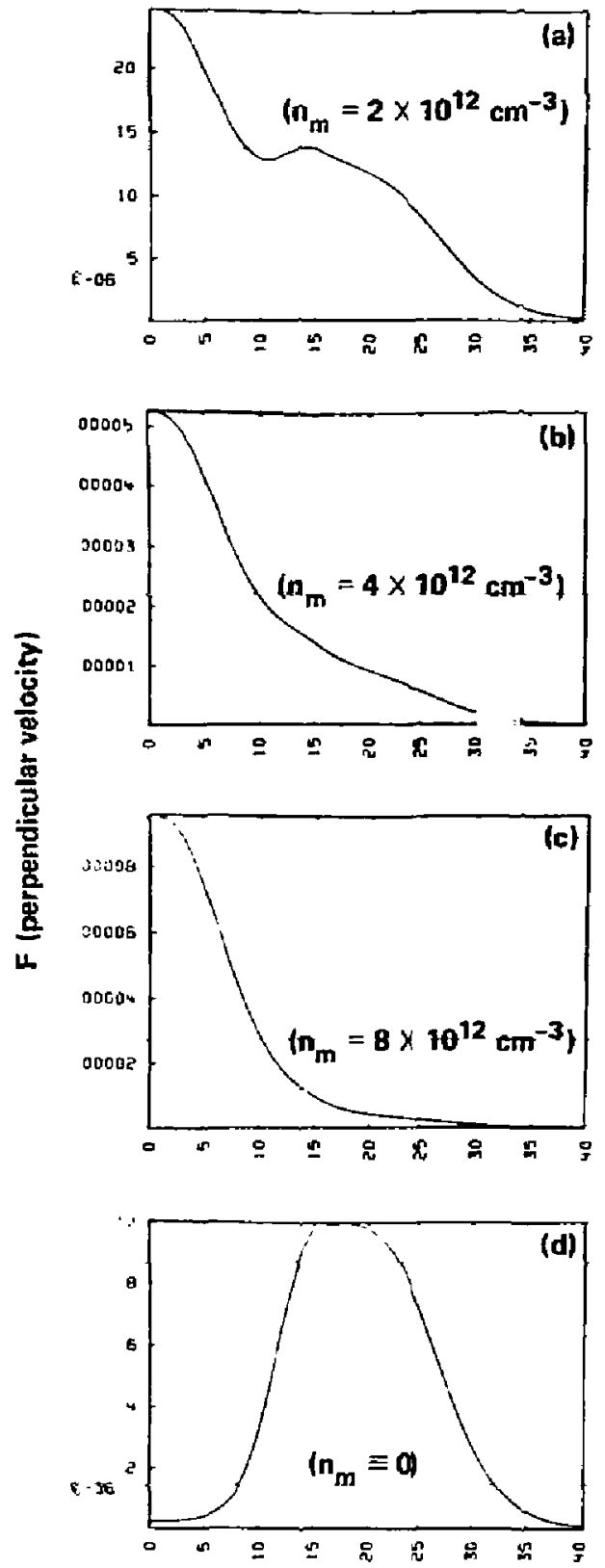

Perpendicular velocity $\left(10^{7} \mathrm{~cm} / \mathrm{s}\right)$

Fig. E-6. Distribution of perpendicular velocities at anchor midplane for four values of $n_{m}$. 

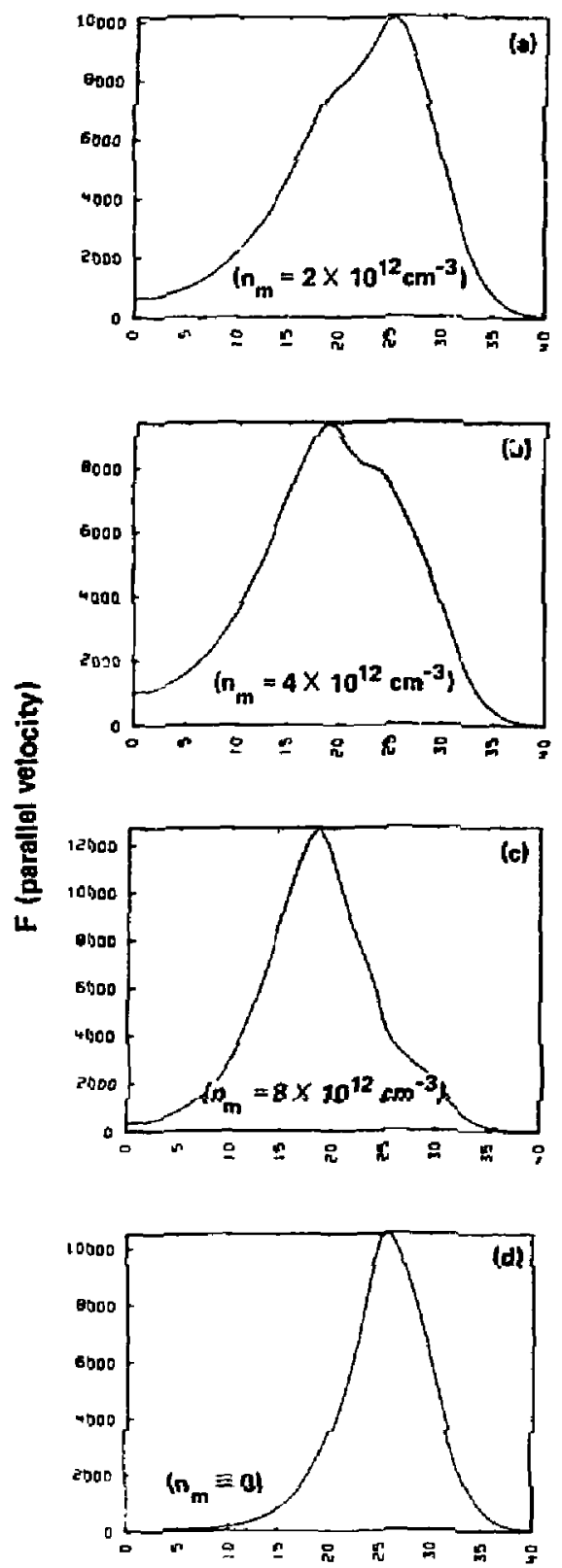

Parallel velocity $\left(10^{7} \mathrm{~cm} / \mathrm{s}\right)$

Fig. E-7. Distribution of parallel velocities at anchor midplane for four values of $n_{m}$ * 
midplane- $v_{1}$ distributions in Fig. E-6 confirms this expectation. If the higher value of the passing-ion density in case $C$ should turn out to be the most plausible, but a larger fraction of sloshing ions is required (e.g., to produce a larger potential peak), then both the HEPB and the sloshing-beam currents would have to be increased relative to the values given in Tables $E-1$ and $\mathrm{E}-2$.

The presence of the warm (passing + trapped) ions in cases $A, B$, and $C$ tends to obscure some of the features of the sloshing ions, so to obtain a clearer picture we examine case $D$ in which there are no warm ions. Here we use the same HEPB as in case $B$ but arbitrarily set the passing-ion density to zero. Results are given in Table E-2 and Figs. E-4 to E-6. The sloshing-ion midplane density for this case is $n_{0}=2.08 \times 10^{12} \mathrm{~cm}^{-3}$; the peak density is 2.84 times higher. The peak, which occurs at a mirror ratio $\psi_{p k}=1.3$, is shifted toward the midplane from the injection point at $\psi_{i n j}=1.5$. This inward shift is due to ion-ion scattering, which tends to broaden the midplane pitch-angle distribution of the sloshing ions. Ions that scatter toward smaller pitch angles escape through the loss cone, while ions that scatter toward $\theta_{0}=90^{\circ}$ are trapped closer to the midplane.

The particle confinement time for the sloshing ions is 1 imited by a combination of charge exchange on the HEPB and axial loss because of ion-ion scattering into the loss cone. This can be seen from the particle balance equation which we write in the form

$$
\frac{\partial N_{S L}}{\partial t}=+J_{\text {ionization }}^{S L}-\frac{N_{S L}}{\left\langle\tau_{C X}^{H E P B_{S}}\right.}-\frac{N_{S L}}{\left\langle T_{\text {end-1 oss }}{ }^{2}\right.} \text {, }
$$

where the line-integrated particle and current densities are

$$
\begin{aligned}
& N_{S L}=\oint \frac{d z n(z)}{\psi(z)}, \\
& J_{\text {ionization }}^{S L}=\oint d z \frac{v_{I}^{S L}(z) n(z)}{\psi(z)} .
\end{aligned}
$$

In steady state, for case $D$, we find that the lifetimes for loss from charge exchange and scattering are comparable, 


$$
\begin{aligned}
& \tau_{\mathrm{CX}}^{\mathrm{HEPB},}=0.65 \mathrm{~s}, \\
& <\tau_{\text {end-10ss }}>=0.88 \mathrm{~s} .
\end{aligned}
$$

The somewhat shorter lifetime for charge exchange is typical of most cases we have considered for sloshing ions in the anchor. 


\section{APPENDIX $F$}

ION MICROSTABILITY

L. D. Pearlstein and G. R. Smith

This section contains assessments of both loss-cone and Alfvern ioncyclotron instabilities and an overall conclusion.

1. LOSS-CONE STABILITY (L. D. Pearlstein)

The background for the material presented here on stability to loss-cone modes was detailed in Phys :CS Basis for MFTF-B, ${ }^{B-1}$ and will not be repeated here. Rather, I will update the physics and the status of the stability to loss-cone modes in the axicell configuration. In this configuration, there are still two cells (the axisymmetric mirror and the quadrupole anchor) with loss-cone distribution of ions. As in the A-cell yin-yang, the axicell ion distribution averaged over the parallel velocity is a monotonic function of the perpendicular velocity and thus is stable to loss-cone modes. After such an average, the yin-yang anchor-barrier ce11, 1ike the A-ce11, does maintain a loss-cone between the midplane and the outboard mirror (see Fig. F-l and its stability must be ascertained.

I begin with a brief description of the modes. In general, the electrons generate the waves while the ions provide the instability mechanism. Stability rests on the control of the ion distributions. An inportant property of these modes are the various space scales. The wayelengths perpendicular to the magnetic field are short $\left(k_{1} \rho_{i} \geqslant 1\right)$ compared to equilibrium scale lengths and, consequently, the eikonal approximation can be used for this variation. Wavelengths along the magnetic field are comparable to equilibrium scale lengths (variations in the magnetic field, density, and the like). Hence, we soive a second-order $0 D E$ along the magnetic field $\mathrm{F}^{-1}$ with $k_{\perp}(\mathrm{s})$, to find the eigenvalues, that is, the standard "balloon" equation. The form of this differential equation follows: 

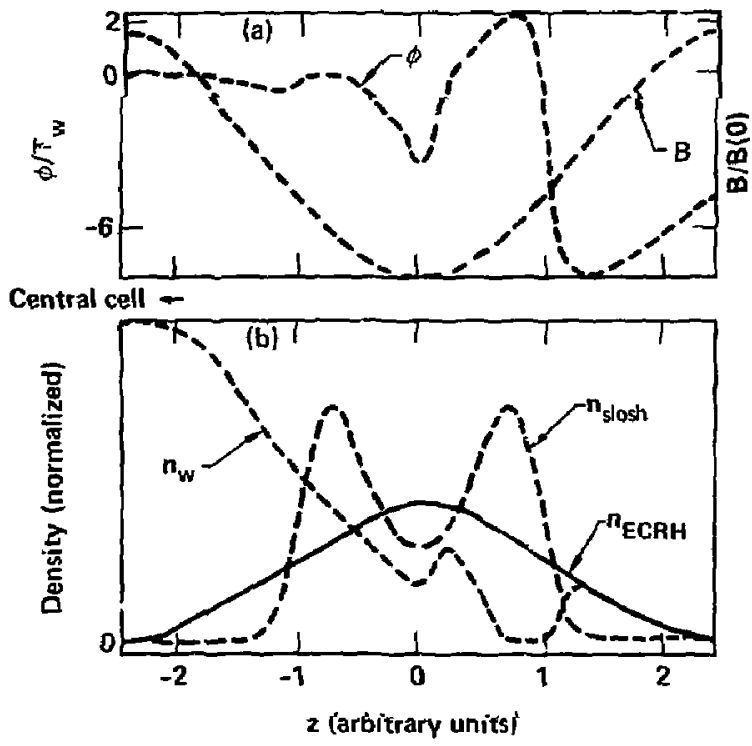

Fig. F-1. Stability of axicell configuration to loss-cone modes: (a) Electrostatic potential $\phi$ and magnetic field $B$ as a function of $z$, distance along the magnetic field; (b) sloshing-jon density, the thermal-ion density, and the magnetically confined ECRH-generated electron density, all shown as functions of $z$. The central cell is off to the left. All densities are normalized to the peak sloshing-ion density. 


$$
\omega_{c i} \frac{d}{d s} \frac{\omega_{p e}^{2}}{\omega^{2} \omega_{c i}} \frac{k_{1}^{2} c^{2}}{k_{\perp}^{2} c^{2}+\omega_{p e}^{2}} \frac{d \phi}{d s}=k_{1}^{2}\left(\phi+F_{e} \phi+F_{i}[\phi]\right),
$$

where

$$
\mathrm{F}_{\mathrm{e}}=\frac{\omega_{\mathrm{pe}}^{2}}{\omega_{\mathrm{ce}}^{2}}+\frac{1}{k_{1}^{2} \lambda_{\mathrm{D}}^{2}}\left[\left(1-\frac{\omega}{\omega}\right)\left(1+\frac{\hat{\beta}}{2}\right)-1\right],
$$

with

$$
\hat{\beta}=\frac{1-\frac{\beta}{2} \phi_{2}-\frac{\omega}{\omega_{c i}}\left(1+\phi_{2}\right)}{1-\frac{\omega}{\omega_{c i}}\left(1+\phi_{2}\right)}
$$

and

$$
\phi_{2}=-\int_{0}^{\infty} d v_{1}^{2} f_{e}\left(v_{1}^{2}\right) \frac{\omega-\omega_{\star} e}{\omega+\omega_{\star} v_{1}^{2} \frac{B}{2}} .
$$

Al1 undefined notation is standard (for definitions see Ref. F-1). Aiso the new form $(\hat{B})$ of the finite $\beta$ term added to the basic equations arises from the $\nabla B$ electron drift with $k_{1} p_{e} \ll 1 . .^{F-2}$ Note that in the 1 imit $T_{e} \rightarrow 0$, we recover the standard result $\hat{\beta}=\beta$. Also $F_{i}[\phi]$ is as defined in Ref. $F-1$. One further addition has been an improved self-cons istent barrier-potential model. F-3

In Fig. F-2 I present results for the yacuum mirror ratio 2.8 , $\beta_{\text {vac }}=0.55$ (actual mirror ratio 4.) for the sloshing-ion profile shown. Also shown is the density of magnetically trapped electrons and the density of thermal ions and the potential, all as functions of $s$. The potential profile shown differs somewhat from that calculated in Sec. II, although the warm-plasma-density profile is quite similar. This difference is due to our approximate model distribution function, which must be an analytic function of mod-B (see Ref. F-3) for the stabilitiy analysis.

In Fig. F-2, stability boundaries are shown for the two worst modes (solid line) of the configuration described above and stability boundaries for a configuration with a $20 \%$ broader ECRH profile (Jashed 1 ine), thus also a more flattened well. The ordinate is the ratio of the thermal-ion density to 


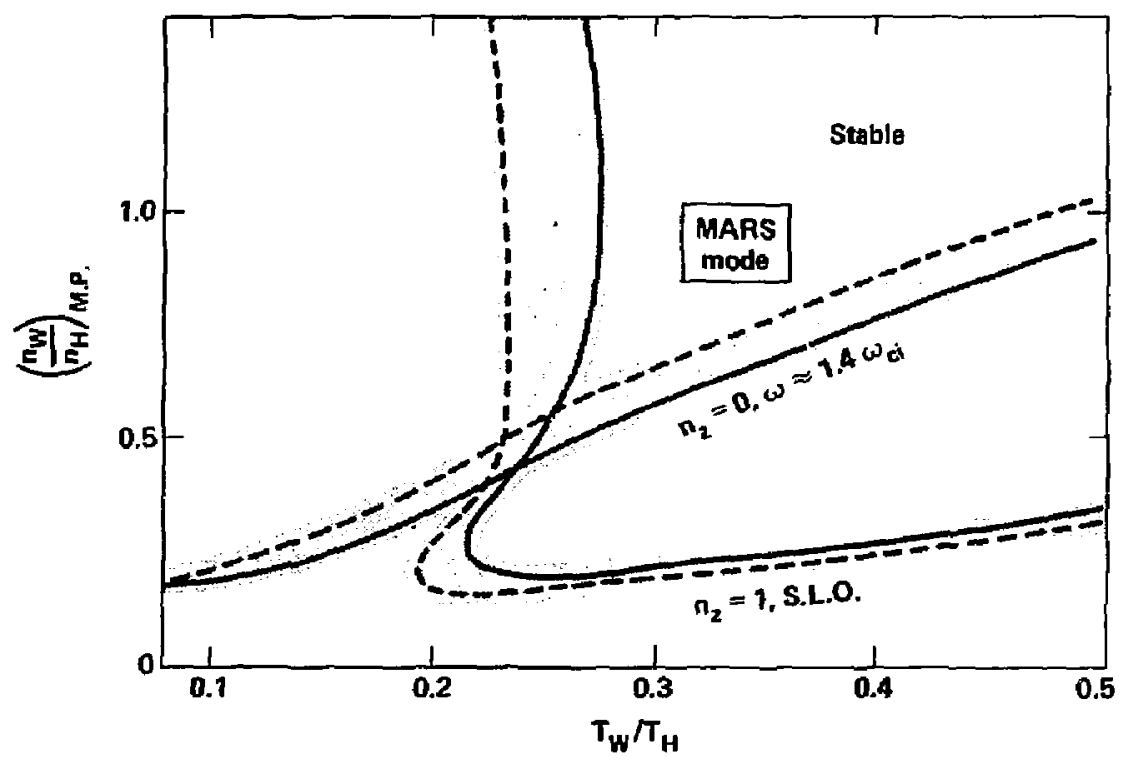

Fig. F-2. Marginal stability for two worst modes (solid lines) and for a configuration with a $20 \%$ broader ECRH profile (dashed lines). The two curves of each set correspond to two magnetically contained electron profiles varying by $20 \%$ (see text). The vertical axis is the ratio of thermal-ion to sloshing-ion density at the mirror midplane. The horizontal axis is the ratio of thermal-ion to sloshing-ion energy, The shaded regions imply the existence of uncertainty in profiles. The square region depicts the nominal operating regime for the MARS mode. 
the sloshing-ion density evaluated at the midplane of the mirror, and tine abscissa is the ratio of the thermal-ion energy to the energy of the sloshing ions evaluated at the midplane of the mirror. The curve labelled $n_{2}=0$ (the lowest axial mode) is the stability boundary for the analog of the drift-cyclotron-loss-cone (OCLC) mode in a symmetric wel1, but here $\omega \approx 1.4 \omega_{c j}$ and is near a resonant frequency in the region of no warm plasma. The curve labelled $n_{z}=1$ (the next mode) is the stability ooundary to the axial loss-cone mode (ALC) computed from straight-line orbits where $\omega>5.5 \omega_{\mathrm{ci}}$. The normal operating regime is denoted by the rectangle shown in the figure.

The basic conclusion of this study is that stable regions for loss-cone driven modes over lap the operating regime of the MFTF-B Axicell configuration. The shaded area on the graph represents the sensitivity to details and is a measure of the uncertainty of the theory. Obviously, a more extreme variation in profiles can expand the shaded area to include the operating point. It should be emphasized that this sensitivity to moderate changes in scale length is primarily a property of the large hole in the perpendicular sloshing-ion distributions, a signature common to both the A-cell and axicell configurations.

Further properties of a marginally stable point are shown in Figs. F-3 and F-4. The marginally stable distribution function averaged over the parallel velocity is shown in Fig. F-3. The values generated from a bounce-averaged Fokker-Planck code (circles) are used as input to the various scenarios. Last, Fig. F-4 shows the magnitude and phase of typical eigenfunctions.

\section{ALFVÉN ION-CYCLOTRON STABILITY (G. R. Smith)}

Each of the operating modes of MFTF-B has at least one mirror cell for which Alfuén ion-cyclotron (AIC) stability must be considered. Here, we give a preliminary assessment of AIC stability in each type of cell. More detailed assessments will be undertaken as Fokker-Planck results for each cell become available. Also, we describe briefly the flexible, model ion distribution function being used for each type of cell.

The various operating modes have two qualitatively different types of mirror cells, those containing sloshing ions and those without a sloshing-ion component. 


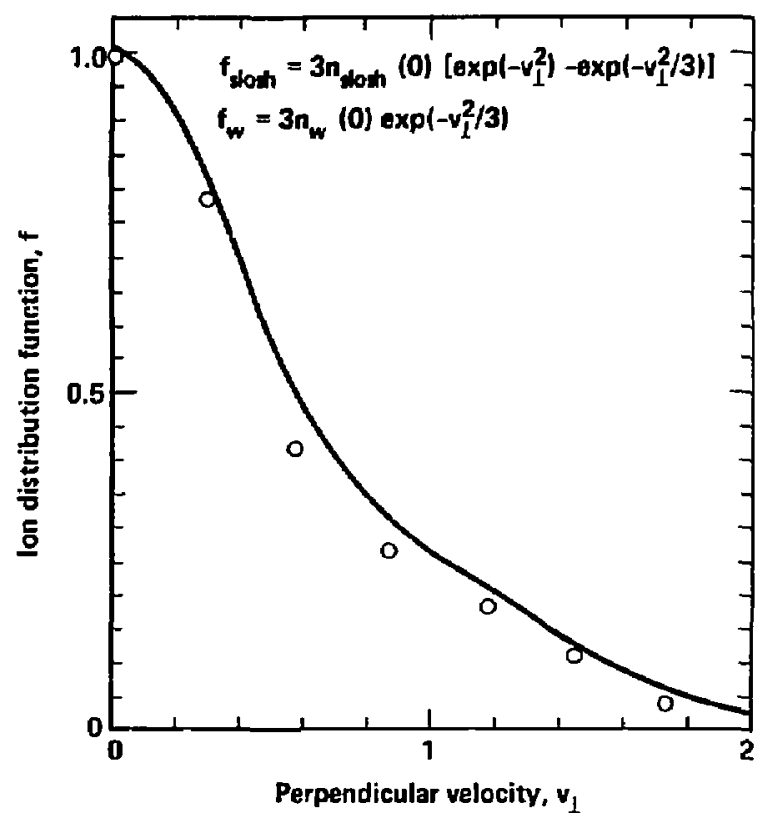

Fig. F-3. Ion-distribution function $f$ as a function of perpendicular velocity at midplane of anchor at marginal stability. The circles depict values obtained from a Fokker-Planck run. 


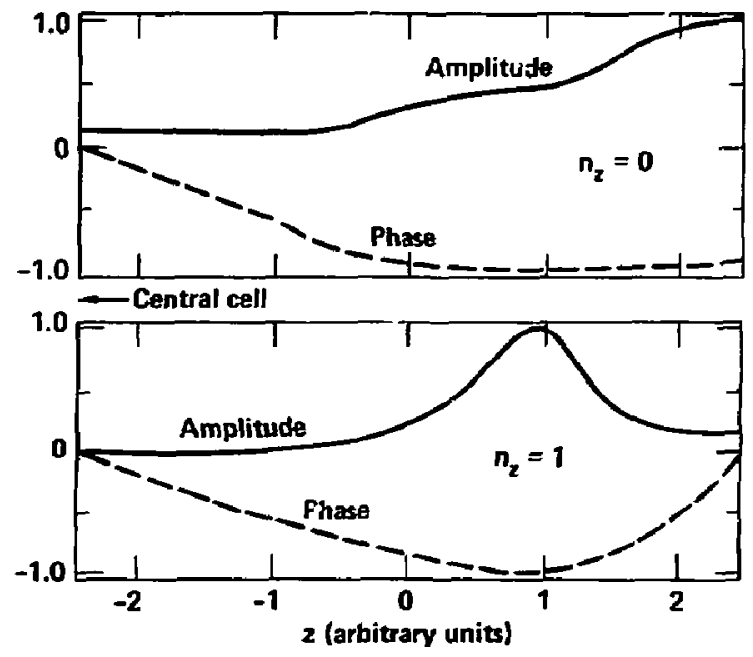

Fig. F-4. The amplitude and phase of the eigenfunction of marginal stability for the $n_{z}=0$ mode and for the $n_{z}=1$ mode. 


\subsection{Anchor-Plug Cell With Sloshing Ions}

For three operating modes (axicell, Kelley, and TMX-U-like) the anchor plug implemented in the yin-yang magnet has a strong sloshing-ion component. Hot electrons provide most of the $B$; the $B$ of the sloshing ions is moderate, of order $10 \%$, a few times the value expected in TMX-U.

In initial calculations of the effert of a sloshing-ion component on AIC instability, a strong stabilizing effect has been discovered. Crudely, the sloshing energy of the ions can be included in calculating the anisotropy $\left\langle v_{1}^{2}\right\rangle\left\langle\left\langle v_{\|}^{2}\right\rangle-1\right.$ that drives the instability. Thus, a sloshing-ion component with mean pitch angle of $45^{\circ}$ contributes weakly, at most, to driving AIC instability.

Because of this strong stabilizing effect, the largest drive for AIC instability may cone from the region where the ions reflect from the inner mirror, since the anisotropy and ion energy (and therefore $B$ ) are both high there. This region is fairly short $(\leq 100 \mathrm{~cm}$ ) in terms of the typical unit of length (inverse wavenumber) for AIC instability

$$
\frac{c}{\omega_{p i}}=\frac{2.3 \times 10^{7}}{2}\left(\frac{\mu}{n}\right)^{1 / 2} \mathrm{~cm} \text {, }
$$

where $Z$ and $\mu$ are the ion charge and mass numbers and $n$ is in cubic centimeters. For MFTF-B, $Z=1, \mu=2, n \approx 6 \times 10^{12}$, and $\mathrm{c} / \omega_{\mathrm{pi}} \approx 15 \mathrm{~cm}$. The shortness of this region of high sloshing-ion density shows the need for calculations that take into account the axial equilibrium variations. Detailed calculations including sloshing ions and axial variations are necessary to refine the paraneters of this anchor-plug cell.

\subsection{Cells Without Sloshing Ions}

\subsubsection{Anchor-Plug Cell of TMX-Like Operating Mode. This cell, also} implemented in the yin-yang magnet, has high ion $B$, like the successful $2 X I I B$ experiment. However, unlike $2 X I I B$, the stabilizing effect of finite length is negligible in MFTF-B since for $n \approx 10^{14}$,

$$
\frac{c}{\omega_{p i}} \approx 4 \mathrm{~cm} \ll L_{p l a s m a} \sim 150 \mathrm{~cm} \text {. }
$$


Counteracting the destabilizing effect of larger plasma size is the potentially stabilizing effect of larger mirror ratio in MFIF-B. The vacuum mirror ratio of 2 in 2XIIB was not fully utilized because of the maintenance by electron drag and charge exchange of an ion distribution much shorter than the mirror-to-mirror distance. If means can be found in MFTF-B to build an ion distribution that fills this cell of vacuum mirror ratio 3 , AlC stability can probably be achieved in spite of the long plasma length.

2.2.2 Axicell. The axicell operating mode of MFTF-B (and of the similar MARS reactor study) contains an axisymetric mirror cell with moderate ion $B$ (comparable to the TMX end ce11). In this respect, the axicell is much superior to the yin-yang cell of the A-cell design of MFTF-B, which requires $B$ to be many times that achieved in TMX. The smaller mirror ratio of the axicell ( 1.5 on the central-cell side) allows confinement of only a rather anisotropic distribution. The anisotropy could, however, be somewhat less than in TMX and in the A-cell design, which (1ike 2XIIB) did not utilize their full mirror ratios. An estimate of the anisotropy limit can be obtained by adapt ing the convective-absolute boundary calculation of Hedrick F-4

$$
\frac{\beta_{1}}{\beta_{\|}} \approx 1.9 \mathrm{~B}^{-1 / 2} \text {. }
$$

For $B$ of $20 \%$, this predicts that absolute instability can be avoided if

$$
\frac{B_{1}}{B_{\| I}} \leq 6 \text {, }
$$

which should be achievable. This calculation neglects the stabilizing effect of the central-cell ions that seem to be present in TMX. In MFTF-B the effect may be stronger than in TMX, because the central-cell icns have significant density throughout the axicell.

The axicell thus seems more stable than the yin-yang of the A-cell design.

2.2.3 Central Cell for Kelley Operating Mode. In this operating mode, which is similar to that of the Technology Development Facility (TDF) strong neutral-beam injection and short confinement time can lead to a significantly non-Maxwellian ion distribution in the central cell. Furthermore, high $B$ is 
required, and the plasma is extremely long in units of $c / \omega_{p i}$, which may imply that suppression of absolute AIC instability is not adequate to avoid deleterious effects; convectively growing waves might heat and trap ions in the bad-curvature transition region. Aneliorating these effects is the very large vacuum mirror ratio of about 10 . The large mirror ratio may al low a sufficiently Maxwellian distribution to be built so that AIC instability can be avoided.

\subsection{Mode 1 Inn-Distribution Function}

An ion-distribution function that is very useful for modeling the various cells of a tandem-mirror machine leads to a numerically tractable dispersion relation for Alfuén ion-cyclotron instability, as descrión in this section.

The ion-distribution function $f$ is a superposition of distributions $f_{j}$ of one or more ion components (e.g., sloshing, passing, trapped):

$$
f(\underline{v})=\sum_{j} f_{j}(\underline{v}) .
$$

The following two models for $f_{j}$ allow a large variety of mirror cells to be studied. The first model has $f_{j}$ separabie in perpendicular and parallel velocity $v_{1}$ and $v_{11}$, while the second has $f_{j}$ separable in speed $v \equiv\left(v_{1}^{2}+v_{11}^{2}\right) 1 / 2$ and pitch angle $\phi \equiv \tan ^{-1}\left(v_{1} / v_{\|}\right)$. Thus,

$$
\begin{aligned}
& f_{j}(\underline{v})=A_{j} f_{\perp}\left(v_{1}\right)\left\{\exp \left[-\alpha_{j}\left(v_{\|}-u_{j}\right)^{2}\right]+\left(v_{\|}+-v_{\|}\right)^{\prime}\right\}, \\
& f_{j}(\underline{v})=F_{j}(v) G_{j}(\phi), \quad G_{j}(\phi)=G_{j}(\pi-\phi) .
\end{aligned}
$$

Both models have the required symmetry under $v_{\|}+=v_{\|}$. For perpendicular wave number $k_{\perp}=0$, the form of $f_{1}$ does not enter the dispersion relation, only the density and temperature moments enter. Even for $k_{\perp} \neq 0$ the first mode 1 allows both velocity-space integrals to be done analytically.

For the second model, a particular class of $F_{j}(v)$ allows one velocity-space integral (speed) to be done analytically for $k_{1} a_{j} \lesssim 1$, where $a_{j}$ is the mean gyroradius of ion component $j$ : 


$$
F_{j}(v)=C_{j} v^{2 l} j \exp \left(-a_{j} v^{2}\right), \quad \ell_{j}=0,1,2, \ldots .
$$

For $k_{\perp}=0$, a rather simple form of the dispersion relation results even for the model $f_{j}=F_{j}(v) G_{j}(\phi)$. Suppressing the subscript $j$ for brevity, we can write the dispersion relation for a plasma with cold electrons as

$$
\begin{aligned}
& 0=D(\omega, k)=k^{2} c^{2}-\omega^{2}+\frac{\omega_{p e^{2}}^{2}}{\Gamma_{e} T}+D_{i}(\omega, k), \\
& D_{i}=\omega_{p i}^{2}\left(1+\frac{2 \pi^{1 / 2}}{\Gamma(\ell+T .5)} \int_{\phi_{105 s}}^{\pi / 2} d \phi \tan \phi G(\phi)\right. \\
& \left.\left\{\sin \phi \tan \phi\left[(2+\ell) z_{3+2 \ell}-z_{5+2 \ell}\right]-\frac{\Omega \alpha^{1 / 2}}{k} z_{2+2 \ell}\right\}\right),
\end{aligned}
$$

where the generalized plasma dispersion functions,

$$
z_{m}(z) \equiv \pi^{-1 / 2} \int_{-\infty}^{\infty} d u \frac{u^{m} \exp \left(-u^{2}\right)}{u-z}
$$

have argument

$$
z=\frac{\omega-\Omega}{k \cos \phi} \alpha^{1 / 2}
$$

Here, $\Omega$ is the ion-cyclotron frequency. These functions can be computed accurately and rapidly. Only a single integral (over pitch angle $\phi$ ) must be done numerically. Nonzero $k_{1} \leqslant a_{j}^{-1}$ does not force the numerical evaluation of both velocity-space integrals but merely introduces a determinant with elements no more complicated than $D$ shown above.

\section{CONCLUSION}

The conclusion of this study is that both the A-cell and axicell configurations look the same with regard to ion microstability. Further, provided the predicted operating parameters can be achieved and some care is taken in forming the ion distributions, we anticipate that the plasma will be stable to these modes. 


\section{REFERENCES}

F-1. D. E. Baldwin, B. G. Logan, and T. C. Simonen, Physics Basis for MFTF-B, Lawrence Livermore National Laboratory, Rept. UCID-18496, Part 2 (1980) Sec. IV.E.

F-2. R. C. Davidson, N. T, Gladd, C. S. Wu, and J. D. Huba, Phys. Fluids 20, 307 (1977)

F-3. L. D. Pearlstein and W. M. Nevins, The Barrier Potential Model, Lawrence Livermore National Laboratory, Rept. UCID-19159 (August 1981).

F-4. C.L. Hedrick, Convect ive-Absolute Boundary for Whist ler Modes, Oak Ridge National Laboratory, Rept. ORNL-TM-3143 (Sept. 1970). 
. 


\section{APPENDIX G}

\section{ELECTRON MICROSTAB:LITY}

Y.-J. Chen, W.M. Nevins, and G.R. Smith

\section{INTRODUCTION}

The hot-electron plasmas in tandem mirrors miy be unstable at frequencies comparable to the electron-cyclotron frequency $\Omega$. By studying these instabilities we hope to prevent deleterious effects by learning how to operate expe: iments without strong instabilities.

An early review of microinstabilities of ECRH plasmas was provided by Guest and Sigmar. ${ }^{G-1}$ The instabilities that led to the most stringent constraints on plasma parameters were the electromagnetic whistler instability and the electrostatic "upper-hybrid loss-cone" (UHLC) instability. Later work on the whistler instability shows that relativistic effects are significanily stabilizing even for mean electron energies much less than the rest energy. G-2 to G-4 The UHLC instability was the subject of a detailed study for plasma parameters appropriate to the earth's magnetosphere. ${ }^{6-5}$

In this previous work ${ }^{G-1}$ to $G-5$ it was found that the wavelengths associated with these high-frequency electron modes are short in comparison to typic..l macroscopic scale lengths of the plasma. Hence, requiring stability in the case of these modes tends to constrain the electron-distribution function rather than the configuration of an experimental plasma.

In this article we describe our present numerical study of instabilities of ECRH plasmas. Our work improves on earlier work by more accurately modeling the electron-distribution functions suggested by recent Fokker-Planck studies of electron-cyclotron-resonance heating (ECRH) in a magnetic-mirror field. $G-6, G-7$

\section{MODELING THE ELECTRON DISTRIBUTION}

Fokker-Planck studies of ECRH plasmas in the TMX-Upgrade Experiment have found distributions $f(\underline{v})$ like the one shown in Fjg. G-1, G-6,G-7 The distribution for MFTF-B will be similar to that shown in Fig. G-I. The monents 


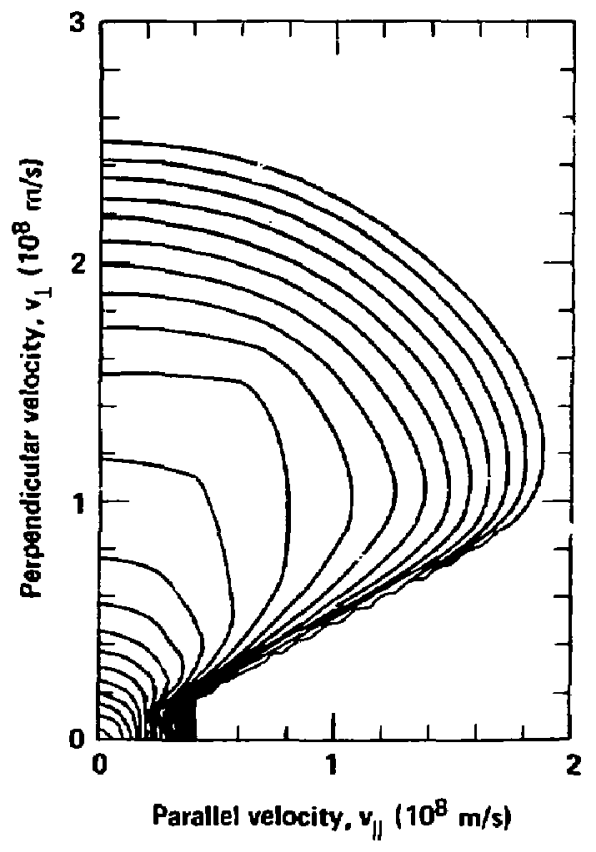

Fig. G-1. Contour plot of electron distribution found in Fokker-Planck studies of the ECRH plasma in the TMX-U experiment. The total electron density is given by $\omega_{p}^{2} / s^{2}=1.55$. Contours are logarithmically spaced, adjacent contours being separated by a factor of iwo. 
$f_{1} \equiv \int d v_{\|} f(\underline{v})$ and $f_{\|} \equiv 2 \pi \int v_{1} d v_{1} f(\underline{v})$ are monotonically decreasing functions of perpendicular and parallel energy, respectively. It does not follow, however, that loss-cone instabilities are absent, because the distribution in Fig. G-1 is poorly approximated by $f_{1} f_{11}$, i.e., $f(\underline{y})$ is not a separable function of perpendicular and parajlel energies. Separable distributions have been used in most previous studies; exceptions are the whistler-instability studies of Scharer ${ }^{6-8}$ and Jacquinot and Leloup. ${ }^{6-2}$ Loss-cone instabilities with finite $k_{\| I}$ can be driven by wave-particle interactions at $v_{\|}=(\omega-n \Omega) / k_{\|}$, along which the perpendicular-velocity distribution is inverted for appropriate $n$, $\omega$, and $k_{\| *}$ Here, $n$ is an integer, $\omega$ is the wave frequency, ano $k_{\|}$is the parallel component of the wavevector. Failure to recognize the nonseparability of $f(v)$ would lead to overly optimistic stability assessments for loss-cone instabilities. Therefore, we have developed the following nonseparable model for the distribution function of an ECRH plasma.

We superpose a number $N$ of electron components with various parameters:

$$
f(\underline{v})=\sum_{s=1}^{N} f_{s}(\underline{v}) .
$$

Each species has a separable distribution

$$
f_{s}(\underline{v})=\frac{n_{s}}{\pi^{3 / 2} q_{1, s^{2} s}^{2} ! \alpha_{11, s}}\left(\frac{v_{1}}{\alpha_{1, s}}\right)^{2 \ell} \exp \left(-\frac{v_{1}^{2}}{\alpha_{1, s}^{2}}-\frac{v_{11}^{2}}{a_{1, s}^{2}}\right) .
$$

By appropriate choices of the densities $n_{s}$, thermal speeds $\alpha_{1, s}$ and $\alpha_{11, s}$, and indices $\ell_{s}$, we achieve a good reproduction of $F i g . G-1$, as shown in Fig. G-2.

Superposing separable distributions to model a nonseparable distribution al lows us to avoid numerical velocity-space integrations, a considerable computational advantage. The distribution in Eq. G.2 is precisely that used by Callen and Guest, ${ }^{G-9}$ who provided all the formulae necessery for numerical calculation of the dielectric function $D(\omega, \underline{k})=\operatorname{det}[\mathrm{D}(\omega, \underline{k})]$, whose roots describe the small-amplitude waves of a collisionless plasma in a uniform magnetic field, $\underline{B}_{0}$.

We have written a computer code that solves the fully electromagnetic dispersion relation $D(\omega, \underline{k})=0$ for the non-separable model distribution given by Eqs. G.1 and G.2. The direction of wave propagation $\underline{k}$ with 


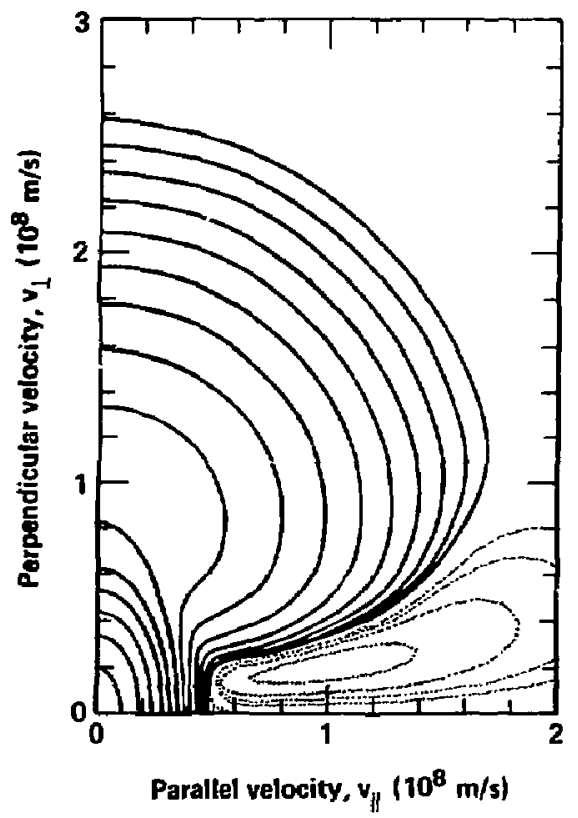

Fig. G-2. Model electron distribution formed by superposing five separable distributions (see Eq. G.2). The logarithmically spaced contours are separated by a factor of two. The plasma frequencies $\omega_{p}$, perpendicular temperatures $T_{1} \equiv(\ell+1) \mathrm{ma}_{1}^{2} / 2$, parallel temperatures $T_{\|} \equiv m \alpha_{\|}^{2} / 2$, and indices $\ell$ are given by $\omega_{p}^{2} / \Omega^{2}=0.64,0.92,-0.037,0.046$, and -0.018 , respectively; $T_{1}=3,40,12,12$, and $4 \mathrm{kev} ; T_{\| 1} / T_{1}=0.3,0.35,4,2$, and 6 ; and $\ell=0,1,1,1$, and 1 . Dotted contours give unphysical negative values of the distribution function. The spacing between adjacent dotted contours is also by a factor of two. The magnitude of the most negative value of the distribution function is less than $1 / 2000$ th of the most positive value. 
respect to $\underline{B}_{0}$ can be arbitrary, but the plasma is assumed to be spatially uniform and nonrelativistic.

The uniform-plasma approximation is a reasonable first approximation because high-frequency instabilities have wavelengths much shorter than typical equilibrium scale lengths of the plasma. The nen-relativistic approximation is known to be pessimistic for the whistler instability: Gladd has found growth-rate reductions of a factor of two or more for mean electron energies of $250 \mathrm{keV}$, one-half the rest energy. ${ }^{\mathrm{G}-3,6-4}$ Relativistic effects are stabilizing only for instabilities with $\omega<\Omega$. Nevertheless, at the 200-600 keV energies envisioned for MFTF-B, even the UHLC instability, which has $\omega>\Omega$, will have to be treated relativistically. The results in the next section were found with the non-relativistic approximation.

\section{NUMERICAL RESULTS}

Only two instabilities, the whistler and upper-hybrid loss-cone modes, are found in a plasma with the non-separable model electron distribution shown in $\mathrm{Fig.} \mathrm{G-2} \mathrm{by} \mathrm{applying} \mathrm{the} \mathrm{Nyquist} \mathrm{technique} \mathrm{thoroughly} \mathrm{over} \mathrm{the} \underline{k}$ plane. We have used our computer code to calculate the real frequencies and temporal growth rates of these instabilities. For the whistler mode the maximum growth rate occurs at $k_{1}=0$ and $k_{1 \mid} c / \Omega=1.41$, where $\omega / \Omega=0.55+i 0.025$. When we double the total electron density without changing the shape of the distribution, we find that the wave vector that maximizes the growth rate shifts to $k_{\|} c / \Omega=1.74$, where the frequency is given by $\omega / \Omega=0.51+i 0.049$.

For the UHLC instability we show in Fig. 6-3 a contour plot of Im $\omega$ in the $k_{1} k_{\|}$plane. The maximum growth rate occurs at $k_{1} c / \Omega=9.9$ and $k_{11} / k_{1}=0.071$, where $\omega / \Omega=1.15+i 0.0054$. These values of $k_{11}$ and $k_{1}$ for the most unstable mode are in line with estimates presented by Guest and Sigmar. ${ }^{-?}$ Note that the growth rate of the UHLC mode goes to zero as $k_{\|}$goes to zero, as expected for our monotonically decreasing $f_{1}$. Doubling the total electron density shifts the maximum growth rate to $k_{1} c / \Omega=10.9$ and $k_{W} / k_{1}=0.13$, where $\omega / \Omega=1.24+i 0.008$.

Defining $\left\langle T_{\|}\right\rangle$and $\left\langle T_{1}\right\rangle$ to be the averaged parallel and perpendicular temperature, respectively, for the distribution given by Eqs. G.l and G.2, our model distribution shown in Fig. G-2 has $\left\langle T_{||}\right\rangle /\left\langle T_{1}\right\rangle=0.32$. We vary $T_{\| 1}$ of all components, keeping ratios of $\mathrm{T}_{11}$ between all five components constant. 


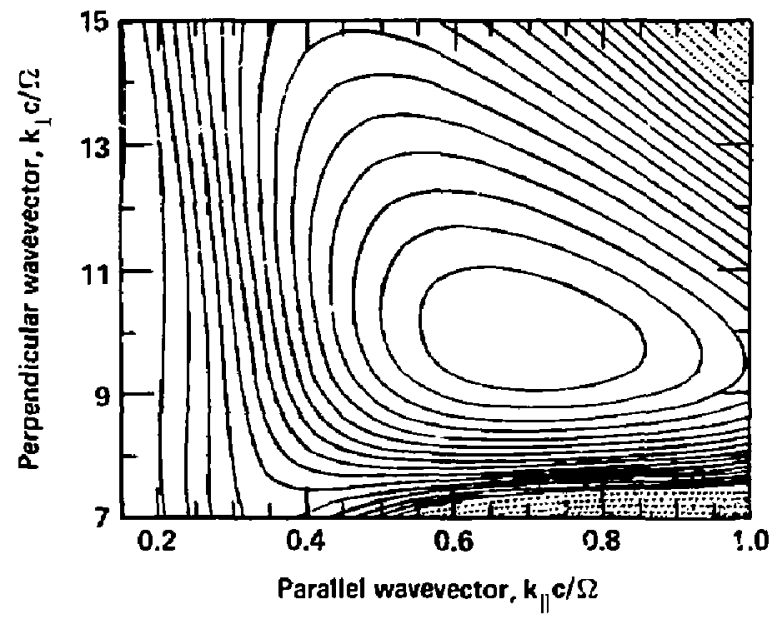

Fig. G-3. Temporal growth rate of the upper-hybrid loss-cone instability for the electron distribution shown in Fig. G-2. Solid contours give Im $\omega \geq 0$, dotted contours give Im $\omega<0$. The interval between adjacent contaurs is $4.0 \times 10^{-4} \Omega$. 
We find that increasing $\left\langle T_{\| \mid}\right\rangle /\left\langle T_{\perp}\right\rangle$ stabilizes the whistler mode, but destabilizes the upper-hybrid loss-cone mode as shown in Fig. 6-4. The maximum growth rates of both modes are less than $0.008 \Omega$ when $\left\langle T_{\| \mid}\right\rangle /\left\langle T_{1}\right\rangle \geq 0.5$. Doubling the $\left\langle T_{1 \mid}\right\rangle\left\langle\left\langle T_{1}\right\rangle\right.$ to 0.64 shifts the maximum growth rates to $k_{11} c / \Omega=0.9$ for the whistier mode, where $\omega / \Omega=0.32+i 0.004$, and $k_{1} c / \Omega=9.25$ and $k_{\| I} / k_{\perp}=0.056$ for the upper-hybrid loss-cone mode, where $\omega / \Omega=1.16+i 0.008$.

Figure G-5 shows curves of constant temporal growth rates in the $\left\langle T_{\| 1}>/\left\langle T_{1}>\right.\right.$ versus $\omega_{p \text { tot }}^{2} / \AA^{2}$ plane, maximized over the $k p l a n e$, for the whist ler and the upper-hybrid loss-cone instabllities. $\omega_{p t o t}^{2}=\sum_{2} \omega_{p s}^{2}$ is proportional to the total electron density. In the $\left\langle\tau_{\|}\right\rangle /\left\langle\tau_{1}\right\rangle$ versus $\omega_{p t o t}^{2} / \Omega^{2}$ plane, the value of $T_{4}$ and $\omega_{p}^{2}$ for each component is varied, such that the ratios of $T_{11}$ and of $\omega_{p}^{2}$ between all five components remain constant. The maximum growth rates of both modes are less than $0.004 \Omega$, if we require that $\left\langle\mathrm{T}_{\|}\right\rangle /\left\langle\mathrm{T}_{1}\right\rangle \geq 0.4$ and $\omega_{\text {ptot }}^{2} / \Omega^{2} \leq 0.55$. Along the growth rate $\gamma_{\max } / \Omega=0.004$ curves, the convective growth length of the whist ler mode varies from $20 \mathrm{~cm}$ to $8 \mathrm{~cm}_{1}$ and that of the upper-hybrid loss-cone mode varies from $3.6 \mathrm{~cm}$ to $1.3 \mathrm{~cm}$, when $\omega_{\mathrm{ptot}}^{2} / \Omega^{2}$ increases from 0.55 to 3.05 and $\theta=1$ tesla.

Figure 6-6 shows the boundary between conditions for absolute and convective whistler instability when the ratios of $\mathrm{T}_{\mathrm{I}_{2}}$ and $\omega_{\mathrm{p}}^{2}$ between all five components remain constant. Since increasing $\omega_{p t o t}^{2} / \Omega^{2}$ increases the required temperature anisotropy $\left\langle T_{\| 1}\right\rangle /\left\langle T_{1}\right\rangle$ for convective instability, increasing the total electron density has a destabilizing effect.

4. SUMMARY

Our work to date has found two instabilities, the whistler and upper-hybrid loss-cone modes, in a plasma with an electron distribution function simjlar to that produced by electron-cyclotron-resonance heating. In the hope of preventing deleteriaus effects in future experiments, we continue to study these instabilities, both in the uniform-plasma approximation discussed here and with effects of spatial nonuniformity. 


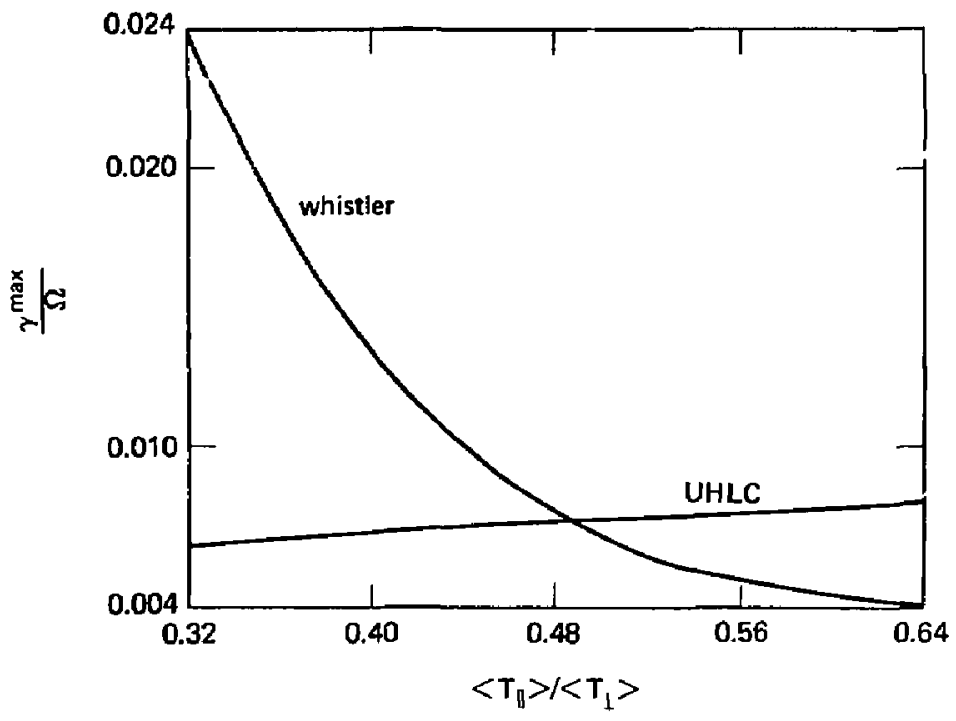

Fig. G-4. Growth rates, maximized over $\underline{k}$, of the whistler and upper-hybrid loss-cone (UHLC) instabilities as a function of the temperature anisotropy $<T_{11}><T_{1}>$. 


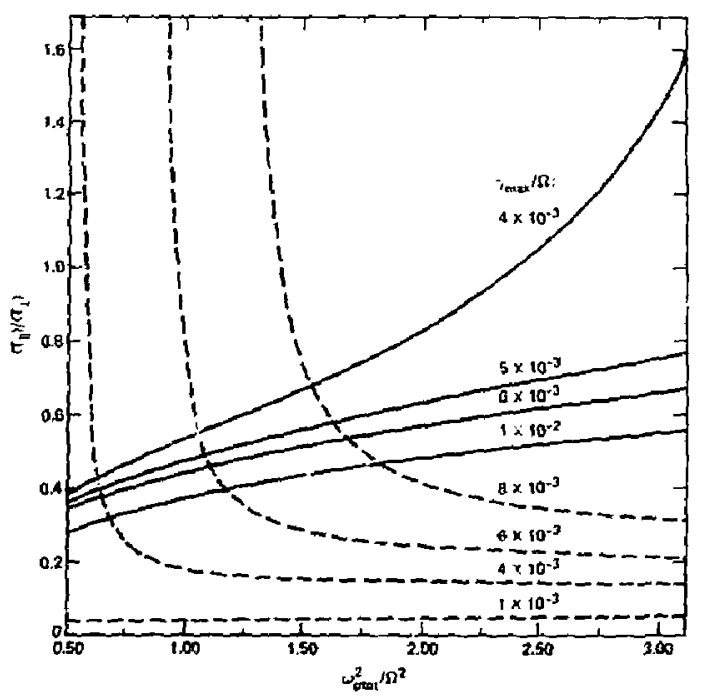

fig. 6-5. Constant-growth-rate curves for the whisicler and UHLC modes. The temperature anisotropy, $\left\langle T_{\|}\right\rangle /\left\langle T_{1}\right\rangle$, is shown as a function of total electron density, $\omega_{p t o t}^{2} / \Omega^{2}$. Growth rates are maximized over the $k$ plane. Solid curves are for the whistler mode; dashed curves for the upper hybrid loss-cone (UHLC) mode.

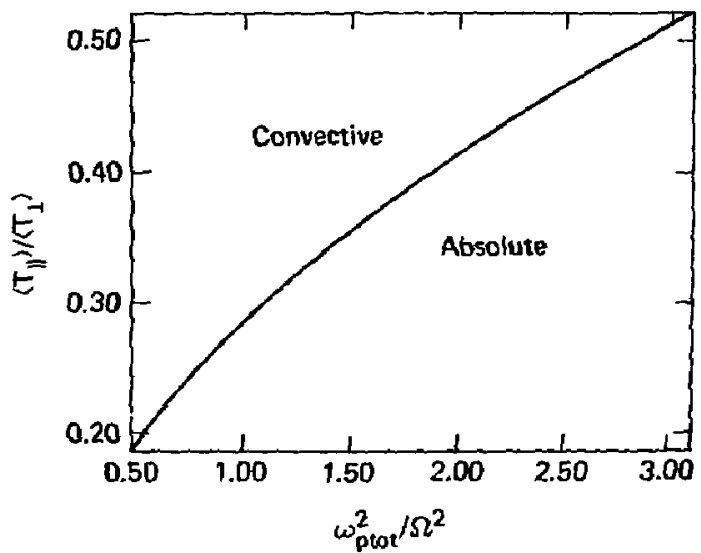

Fig. G-6. Convective-absolute instability boundary for whistler mode. 


\section{REFERENCES}

G-1. G. E. Guest and D. J. Sigmar, kiscl. Fusion 11, 151 (1971).

G-2. J. Jacquinot and C. Leloup, Phys. Fluids 14, 2440 (1971).

G-3. N. T. Gladd, et a1., "Microstability of the EBT Boundary," Sec. IV, in Proc. Workshop on EBT Stability Theory, N. A. Uckan, Ed., Oak Ridge, TH, May 1981.

G-4. H. T. Gladd, "The Whistler Instability at Relativistic Energies," in Proc. 2nd EBT Ring Physics Workshop, M. A. Uckan, Ed., San Oiego, CA, Dec. 1981.

G-5. M. Ashour-Abdalla and C. F. Kenne1, J. Geophys. Res. 83, 1531 (1978).

G-6. Y. Matsuda, H. M. Neyins, and R. H. Cohen, "Electron-Cyclotron Resonant Heated Electron Distribution Functions," in Proc. Horkshop on EBT Stability Theory. N. A. Uckan, Ed., Oak Ridge, TN, May 1981.

G-7. B. W. Stallard, Y. Matsuda, and W. M. Nevins, "Fokker-Planck Calculations of Electron Cyciotron Resonant Heating (ECRH) in Mirror Geometry," in Proc. 2nd EBT Ring Physics Horkshop. N. A. Uckan, Ed,, San Diego, CA, Dec. 1981.

G-8. J. E. Scharer, Phys. Fluids 10, 652 (1967).

G-9. J. D. Callen and G. E. Guest, Nurl. Fusion 13, 87 (1973). 


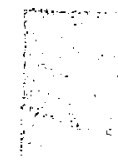

. 


\section{APPENDIX H}

RADIAL TRANSPORT

R. H. Cohen and J. M. Gilmore

We assess here the importance of various mechanisms for transport of ions and ion energy in the solenoid of the proposed MFTF-B Axicell configuration. The transport mechanisms considered are resonant transport and classical diffusion, both resulting from charged-particie encounters and ion-neutral encounters. The principle conclusions are:

- The axicell reduces resonant transport by a factor of order $R^{-1} \exp \left(-\Delta \phi_{p i} / T\right)$ compared to a configuration with the same geodesic-curvature profile but without the axicell betwean the yin-yang cell and the solenoid. (Here $R$ is the ratio of the minimum field strength on-axis in the axisymmetric region to the mid-solenoid value.) However, the simplest axicell magnet designs tend to have large geodesic curvature in the transition region, producing unacceptably large transport. He anticipate the final design for MFTF-B will result in a radial loss rate less than the ax:al loss rate.

- The classical charged-particle energy diffusion rate is about a third of the axial joss rate.

- Ion-neutral transport is a rather weak ( $\mathrm{n \tau} \geq 1.5 \times 10^{14} \mathrm{~cm}^{3} \mathrm{~s}^{-1}$ ) effect in the plasma but is much larger in the halo.

\section{RESONANT TRANSPORT}

As of this writing, the magnetic-field design for MFTF-B has not been finalized. The issue delaying this process, namely the lack of a satisfactory equilibrium, is closely related to resonant transport, as both the parallel current and the radial drift per bounce are proportional to a line integral Sdst] of the geodesic curvature. We have analyzed a preliminary field design and found that the solenoid ions passing into the transition and yin-yang celis satisfy the criterion for intrinsically stochastic transport, thereby implying unacceptably high radial-transport rates. However, a drop in Sdsto by a factor of order five would cause the transport to change from stochastic to banana transport and would be accompanied by a precipitous drop in the diffusion coefficient. Reduction of sdsto by at least such a factor 
is deemed necessary for a good equilibrium. We estimated the banana-diffusion coefficients for the preliminary design to be two orders of magnitude smaller than the stochastic diffusion coefficients, implying a radiai lifetime of the order of the axial lifetime.

The axicell configuration proposed for MFTF-B has a potentially strong advantage over the A-cell magnetic configuration vis-a-vis resonant transport. The axicell mirrors and potential hill confine most of the solenoid ions to a purely axisymetric region, so that, in a bounce time, only a fraction of order $R^{-1}$ exp $\left(-\Delta \phi_{p i}{ }^{\prime T}\right)$ sf the tons experience the quadrupole field, implying a scaling of the diffusion coefficient by the same factor. The scaling with $\Delta \phi_{p i}$, the size of the potential hill in the axicell, is only very crudely given by the exponential factor, as will be explained later.

If we approximate the radial drift per bounce $\Delta r \equiv \cos 2 \beta$ as independent of pitch angle over the range of pitch angles for which particles pass into the nonaxisymetric region, then in the stochastic limit (large electric fiejd, azjmuthal drift per bounce $\Delta B>r / a)$, we can calculate the scaling with $R$. The stochastic particle-diffusion coefficient is ${ }^{H-1}$

$$
D_{0}=\frac{\pi B}{r L_{s} m^{2} n} \int d \varepsilon d \mu a^{2} f
$$

Taking $f$ to be Maxwellian and independent of $v_{\| 1} / v$ over the range $\left(1-R^{-1}\right)^{1 / 2}<v_{\mid f} / y<1$, we find $D_{0}<1-\left(1-R^{-1}\right)^{1 / 2}$ which is proportional to $R^{-1}$ for large $R$. For more moderate electric fields, the diffusion coefficient depends on the velocity space location of individual resonances, as well as the collisionality regime (banana/plateau); the scaling remains $O(1 / R)$, although a precise general scaling cannot be written.

Calculation of resonant transport in the axicell configuration is simpler than in previous configurations, because solenoid ions which pass into the nonsymmetric region all have $v_{11} / v=1$ at the midplane of the solenoid, and all reflect off of the potential hill in the yin-yang cell. Thus, the general expression for the change of flux coordinate $\alpha$ in a bounce,

$$
\Delta a=2 \frac{m c}{e} \int_{0}^{s_{t}} d s\left(v_{11}+v_{1}^{2} / 2 v_{11}\right) 10 \text {; }
$$


where $t D$ is the geodesic curvature, $s=0$ denotes the solenoid midplane, and $s_{t}$ denutes the turning point; becomes

$$
\Delta \alpha=\left\{\begin{array}{cc}
\frac{2 m c v_{t}}{\mathrm{e}} \int_{0}^{s} \phi s t \sigma, & \left(v_{\|} / v_{s}\right)>(1-1 / R)^{3 / 2}, \frac{1}{2} m v_{s}^{2}>\Delta \phi_{p i} \\
0 & \text { elsewhere }
\end{array}\right.
$$

where now $s_{\phi}$ denotes a point part way up the side of the potential hill in the yin-yang cell. In Eq. H.3 and henceforth, a subscript $t$ denotes evaluation of a quantity in the transition region, whereas a subscript s denotes a solenoid value.

Equations $H .2$ and $H .3$ are usually evaluated along a field line, a procedure that is only accurate as long as the azimuthal drift $\Delta \beta_{e}$ during the time an ion spends in the transition and yin-yang regions is negligisle. In the opposite limit $\Delta \beta_{\mathrm{e}} \gg \mathrm{l}$, $\Delta \alpha$ should become exponentially small, in $\Delta \alpha \propto-\Delta \beta_{e}$. Following in the spirit of Raf. H-3, we assume Eq. H.2 is valid for $\omega_{B t} L_{e} / v_{1}<1$, where $\omega_{B}$ is the azimuthal drift frequency, and take

$$
\Delta \alpha=0 \quad, \quad \omega_{B t} L_{e} / v_{\|}>1 \text {. }
$$

Here $L_{e}$ is an effective length for the transition region; a plot of vs $s$ for the preliminary MFTF- $B$ design iridicates that most of the contribution to fds comes from a narrow spike about $2 \mathrm{~m}$ in length in the transition region; hence we take $L_{e}=2 \mathrm{~m}$ in the numerical calculations described below.

The integral in Eq. H.3 is available as output in the TEBASco equilibrium code; hence we can est imute $\Delta \alpha$ without numerically calculating drift orbits. (Furthermore, use of Eqs. H.3 and H.4 with $\omega_{B}=\omega_{E X B}$ and approximating the bounce $t$ ime by $t_{\|}=L / v_{\|}$when $L$ is the distance between yin-yang centers, allows the integrals in the resonant-banana, resonant-plateau and stochastic diffusion coefficients ${ }^{\mathrm{H}-1, \mathrm{H}-2}$ to be evaluated analytically. These results will be described in a separate cormunication.)

The r.riterion ${ }^{\mathrm{H}-\mathrm{T}}$ for stochastic transport can be approximately in itten as $K=2 \mathrm{a} \Delta B / \mathrm{r}>1$; here, $\Delta B$ is the azimuthal drift in a bounce. Approximating $\triangle B$ by EXB drift alone, this becomes

$$
E>\frac{T_{i}}{e r} \frac{r^{3}}{\rho-a} \text {. }
$$


From Eq. H.3 and TEBASCO output for the vacuum field of case EG224 (generated by the EFFI code, , we iind $S d s+D=24 \mathrm{~cm}$ at : $=21 \mathrm{~cm}$, implying that with energy of $45 \mathrm{keV}$ in the transition region (15 keV in the central cell), $a / r=0.47$ for ions. Then, for the reference MFTF-B parameters of Table 2.1-1 (MARS mode): we find stochasticity for $E>70 \mathrm{~V} / \mathrm{cm}$. For $E \sim \mathrm{T}_{\mathrm{e}} / \mathrm{er}$, the design studied satisfies $\mathrm{H} .5$ by about a factor of five. Integration of Eq. H. 1 using Eqs. H.3 and H.4 gives

$$
\left.D_{0}=\frac{a^{2}}{4 \pi^{1 / 2} L R}\left(\frac{2 T}{m}\right)^{1 / 2} \exp \left(-\Delta \phi_{p i} / T\right\rangle G i x_{0}, x_{l}\right),
$$

where $x_{0}=(\mathrm{eEr} / \mathrm{T})^{2}\left(2 \mathrm{~L}_{\mathrm{e}} \rho / \pi \mathrm{r}\right)^{2}, \mathrm{x}_{1}=\Delta \phi_{\mathrm{pi}} / \mathrm{T}$, and

$$
G= \begin{cases}x_{1}^{2}+2 x_{1}+2+\Phi\left(x_{1}+1\right) & x_{1}-x_{0}>x_{1} / R \\ \left(x_{1}-x_{0}\right)\left(x_{1}-2\right)+2+\Phi\left(x_{1}-x+1\right)-g+h, & 0<x_{0}-x_{1}<x / R \\ \exp \left(x_{1}-x_{0}\right)[2+\Phi+h]-g & x_{0}>x_{1} .\end{cases}
$$

Here, $x_{L}=x_{0}\left(1-R^{-1}\right)^{-1}, \Phi \equiv T^{-1} \times$ (potential difference between solenoid and transition region),

$$
g=\exp \left(x_{1}-x_{2}\right)\left[\left(x_{2}-x_{0}\right)\left(x_{2}-2\right)+2+\Phi\left(x_{2}-x_{0}+1\right)\right],
$$

and $h=R^{-1}\left(x_{2}^{2}+2 x_{2}+2\right)$. For MFTF-B parameters this gives $D_{0} \simeq 5.5 \times 10^{-4} \mathrm{~cm}^{2} \mathrm{~s}^{-1}$ and a corresponding lifet ime of order $10^{-2} \mathrm{~s}$. This time is shorter than the classical time to scatter into the loss cone, $\tau_{j i} \operatorname{lnR}$. Thus the transport would act to produce a loss-core distribution.

The above estimates for the diffusion rate can be considered as an upper bound. Additional effects could reduce the diffusion rate, at least mildly, in the design studied and perhaps drastically in the final design.

For example, the above estimate is obtained by neglecting the azimuthal drift in the transition region until it is large enough to rotate particles by order $\pi / 2$ in a single transit through the spike in the geodesic curvature. However, before that limit is reached, the azimuthal drift will be large enough to cause particles to drift by order $\pi / 2$ between their pre- and post-bounce passages through the spike in 10 . The radial displacements accompanying these successive pa: es no longer add in phase, as previously 
assumed. At least, the successive phases will be effectively random, reducing the (stochastic) transport by about a factor o: two. Also, for a (perhaps uninzeresting) range of parameters, the orderly variation of the phase change with radius can lead, as in Ref. $\mathrm{H}-7$, to regions of stochasticity separated by annular nonstochastic bands. The radial lifetime would then be approximately determined by the time to diffuse (under the influence of collisions) across the nonstochastic bands.

A more important effect is that reduction of $\mathrm{SdsH}$ by a factor of order five causes the transport to become resonant rather than stochastic; such a reduction is required for production of a satisfactory equilibrium. Because the collisionality in MFTF-B is such that resonant transport is in the banana regime, the transition to nonstochastic dynamics can produce an order-of-magnitude change of two in the transport rate, as the following example shows. The precise reduction in fostl $r$ squired is uncertain, as Eq. H.3 is only an estimate of the stochasticity criterion: the stochasticity parameter $K$ is actually $K=2 a \partial \Delta \beta / \partial r$ and so depends on second derivatives of the potential and magnetic field; and the stochasticity boundary for an arbitrary dynamical map is only approximately $k=1$. (It is clear, however, that particles with $a / r \sim 1 / 2$, as in the studied design, are stochastic). The stochasticity boundary will be studied in more detail numerically.

To illustrate the difference between stochastic and nonstochastic diffusion rates, we evaluate the nonstochastic resonant transport for the preliminary design. In Fig. H-I, we plot numerical values for the resonant diffusion coefficients $D_{0}, D_{1}$, and $D_{2}$ and the lifetime estimate $\tau_{2}$, as defined by Eq.s. '4. $1, \mathrm{H.2}$, and $\mathrm{H.9}$ of Ref. H-4. The diffusion coefficients are obtained by numerical integration of the smaller of the banana and plateau integrands, as described in Ref. H-5. The acceleration and change in $\omega_{B}$ that is due to the axial variation of the ambipolar potential is neglected. We use Eqs. H.3 and H.4, with both VB and ExB contributions to $\omega_{B}$. For all data shown, the collisionality regime is banana. The electric fields $E_{1}$ and $E_{2}$ in Fig. $H-1$ correspond to $\tau_{e} / r$ and- $\phi_{c} / r$, respectively. We see that, except for the narrow spike arjund $E=200 \mathrm{~V} / \mathrm{cm}$, the lifetime $\tau_{2}$ is always greater than about $1 \mathrm{~s}$, corresponding to $\mathrm{nt}>5 \times 10^{13} \mathrm{~cm}^{-3} \mathrm{~s}^{-1}$, or about the same order as the axial lifetime. Changing ddslo by a factor a will change the banana lifetime by about $a^{-1 / 2}$. Note that the marked difference beiween 


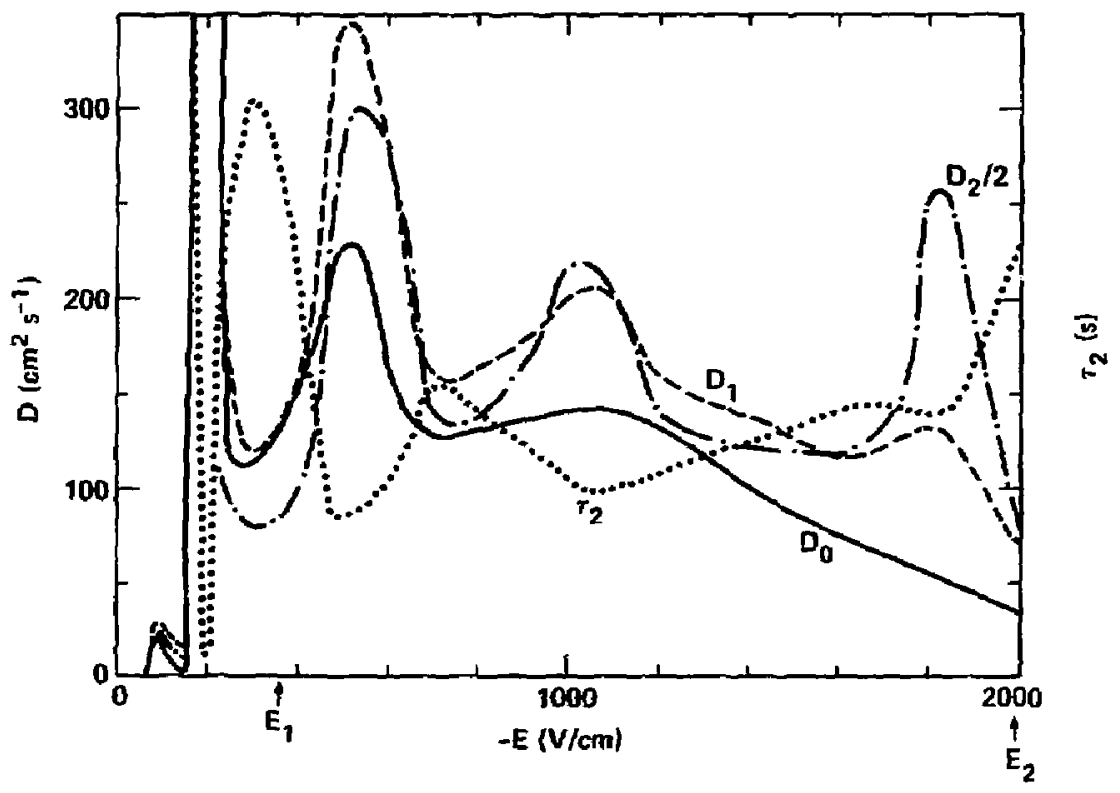

Fig. H-1. Resonant diffusion coefficients $\left(D_{0}, D_{1}\right.$, and $\left.D_{2}\right)$ and lifetime estimate $\left(\tau_{2}\right)$ for MFTF-B preliminary design, all shown as a function of electric field. 
stochastic and resonant lifetimes is due to the low collisionality in MFTF-B. The resonant-plateau and stochastic lifetimes are comparable.

The route being pursued to minimize $\int d s+l$ is to add extra coils, which have the effect of producing two spikes of oppositely signed 15 in the transition region. One can, in fact, thus achieve $f d s t 0=0$ along a field line. However, Ids $v$ 佃 evaluated along a particie orbit will in general not vanish, because of both the azimuthal drift $\Delta \beta_{\mathrm{cr}}$ in a single pass through the transition region and the variation in velocity $v$ from the axial variation of the electrostatic potential. Defining $l_{1}$ to be the integral of 10 over one of the spikes, and $\Delta \phi$ to be the axial change in potential over the region of the spikes, the effective noncancellation of $f d s$ b because of the azimuthal orift and the axial potential variation are, respectively, of order $2 \Delta \beta_{\operatorname{tr}} I_{1}$ and $(\Delta \phi / 2 T) I_{1}$. To ensure nonstochastic dynamics, we must keep these quantities below about $5 \mathrm{~cm} \times(\mathrm{r} / 21 \mathrm{~cm})^{2}$ (i.e., one-fifth of the value of Sdsto in the preliminary design).

The effect of the potential hill in the axicell on resonant transport can be appreciably larger or smaller than $\exp \left(-\Delta \phi_{p j} / T\right)$, depending on the electric field. Because of the large mirror ratio, the principle effect of the potentia1 is to cut off the resonance sum at a resonance number $k=k_{\phi}$ [azimuthal drift per bounce $\equiv(2 k+1) \pi / 2$ ] such that, for $k>k_{\phi}$, all resonant particles are reflected by either the magnetic field or by the potential. Neglecting $\nabla B$ drifts, one has $2 k_{\phi}+1=\left(E / E_{\star}\right)\left(T / \Delta \phi_{p j}\right)^{l / 2}$, when $E_{\star}$ is the electric field for which a thermal particle drifts $\pi / 2$ per bounce. The resonance sum is also cut off when the azimuthal drift in the transition region becomes too large (at a $k$ that is independent of $E$ ). Thus, for large enough $E$, the cutoff due to $\Delta \phi$ becomes irrelevant, while for $E / E_{\star}<\left(\Delta \phi_{p i} / T\right)^{1 / 2}$, resonant transport is effectively eliminated.

For the $\mathrm{R}^{-1}$ scaling and Eq. H.3 to apply, the radial transport of ions magnetically trapped in the solenoid-plus-axicell region must be small compared to that of ions passing to the yin-yang cell. This in turn requires that Sosto in the solenoid-plus-axicell region be small compared to $R(R)$ limes fosto in the transition-plus-anchor region. Here, $h(R)$ equals $R^{-2}$ in the banana regime and equals $R^{-1 / 2}$ in the plateau and stochastic regimes. The inequality for banana transport, which is the more severe one, is satisfied for the design studied. 


\section{CLASSICAL CHARGED-PARTICLE TRANSPORT}

The classical ion-heat flux $Q$ is predominately due to jon-ion scattering, $q \equiv-\left(2 n T / m_{L} R_{L}^{2} \tau_{c i}\right) \nabla T_{i}$, where $\tau_{c i}$ is the ion-ion collision time. Taking $n / n_{0}=T / T_{B}=1-r^{2} / r_{c}^{2}$, where $n_{0}$ and $T_{0}$ denote on-axis values and $r_{c}$ is the central-cell plasma radius, and defining the radial energy lifetime $\tau_{\mathrm{e}}$ by the relation $n_{0} T_{0} / \tau_{e}=-\nabla \cdot 0$, we find $\tau_{E}=r_{c}^{2} \tau_{c i} / 4 \rho_{i}^{2}$, where $\rho_{i}^{2} \equiv 2 T_{i} / m_{i} \Omega_{i}^{2}$. For the MFTF-B parameters of Table H-3, this gives $\tau_{E}=25 \tau_{c j}$. This is to be compared to the lifetime for axial-energy loss, $\tau_{E a}=\left(1+\phi_{i} / T_{i c}\right)^{-1} \tau_{p}$, where $\tau_{p}$ is the Pastukhov (particle) lifetime; for MFTF-B, one has $\tau_{E a} \sim 8 \tau_{c j}$.

For MFTF-E parameters, the classical ion-particle flux is driven, approximately equally by ion-electron and ion-ion $\left[0\left(\rho^{4} / r_{c}^{4}\right)\right.$ terms in flux] scattering; the lifetime is of order $10^{4} \tau_{c j}$ and is, thus, of littie concern.

\section{ION-NEUTRAL TRANSPORT}

The flux of particles arising from ion-neutral collisions, $\Gamma_{i n}$, can be written as:

$$
-\Gamma_{\text {in }}=D_{0 n}\left(\frac{\partial H}{\partial r}+\frac{n e}{T} \frac{\partial \phi}{\partial r}\right)+D_{\text {ln }} \frac{n}{T} \frac{\partial T}{\partial r} \quad ;
$$

where $o_{0 n}=o_{i}^{2} v\left(1+v^{2} / \Omega_{i}^{2}\right)^{-]}$, and we estimate $D_{1 n}=D_{0 n}$. We note

$$
v^{2} / \omega_{i j}^{2} \ll 1 \text { and } v=n_{n}^{F C} \sigma_{i n} \overline{v_{\text {rel }}}, \overline{v_{\text {rel }}}=v_{\text {ion }}, \sigma_{i n}=2 \sigma_{c x} \text {. }
$$

We assume that the density of molecular deuterium neutrals at the plasma edge $(r=40 \mathrm{~cm})$ is $n_{n}=2 \times 10^{10} \mathrm{~cm}^{-3}$. This implies a Franck-Condon neutral density, $n_{n}^{F C}$, of $2.4 \times 10^{7} \mathrm{~cm}^{-3}$ at $r=20 \mathrm{~cm}$, assuming ${ }^{\mathrm{H}-8}$ an attenuation of the Franck-Condons through the halo of two orders of magnitude. Adopting parabolic models for the radial density, temperature, and potential profites gives a flux $\Gamma_{\text {in }}=4.6 \times 10^{13} \mathrm{~cm}^{-2} \cdot \mathrm{s}^{-1}$, corresponding to $n \tau_{\text {in }}=1.45 \times 10^{14} \mathrm{~cm}^{-3} \cdot \mathrm{s}$; the flux further in is smaller yet. On the other hand, in the halo, $r_{i n}=1.4 \times 10^{13} \mathrm{~cm}^{-2} \cdot \mathrm{s}^{-1}$ corresponding to $n \tau_{\text {in }}=7 \times 10^{12} \mathrm{~cm}^{-3} \cdot \mathrm{s}$. 
4. CONCLUSIONS

Stochastic transport would seriously limit the operation of the preliminary design studied. However, reduction of the line-averaged geodesic curvature by a factor of about five should be sufficient to change the transport regime to resonant banana; the resultant radial ni would be about $10^{14} \mathrm{~cm}^{-3} \cdot \mathrm{s}$, which is of the same order as the axial $n \pi$.* Ion-neutral particle transport and ion-jon energy transport have $n t$ values of the order of, but smaller than, the corresponding axial nt values; thus these processes will quantitatively affect profile shapes but not qualitatively alter the machine performance.

* Note added in proof: The constraints on azimuthal drift in the transition region mentioned at the top of page 213 have proved to be serious. For the current (as of $7 / 30 / 82$ ) design it appears necessary to externally control the radial potential profile in the transition region in order to ayoid stochastic transport for the design plasma parameters. 


\section{REFERENCES}

H-1. R.H. Cohen and G. Rowlands, Phys. Fluids 24, 2295 (1981).

H-2. D.D. Rytutov and G.V. Stupakov, Dok. Akad. Nauk SSSR 240, 1086 (1978) [Sov. Phys. Dok 1. 23, 412 (1978)].

H-3. J.R. Myra and P.J. Catto, Phys, Rev. Lett. 48, 620 (1982).

H-4. D. E. Baldwin, B. G. Logan, and T. C. Simonen, Physics Bas is for MFTF-B, LLNL, Report UCID-18496, Part 2, (1980), Sec. IV.C.

H-5. J.H. Foote, A.K. Chargin, R.H. Cohen, T.B. Kaiser, C.V. Karmendy, T.C. Simonen, and R.L. Wong, TMX-Upgrade Magnet System--Design Characteristics and Physics Considerations, LLNL, Report UCRL-86677 (1981).

H-6. R.H. Cohen, "Effect of Magnetically Confined Electrons on Potential Formation in Tandem-Mirror Thermal Barriers", Poster Paper to be presented at the 1982 Sherwood Theory Meeting, Santa Fe, NM, April 26-28, 1982 .

H-7. R.H. Cohen, "Stochastic Motion of Particles in Mirror Machines", in Intrinsic Stochasticity in Plasmas, G. Laval and D. Gresillon, Eds. (Editions de Physiques, Orsay, France, 1979), p. 181.

H-8. G.0. Porter, LLML, private comunication. 
$0 \quad-1$ 


\section{APPENDIX I}

BARRIER POTENTIAL MODEL

\section{D. Pearlstein and $W$. M. Nevins}

We present here a useful model for calculating the variation in the electron density, ion density, and ambipolar potential along magnetic-field lines in the thermal-barrier cell of a tanden mirror. We derive potential proftles in the barrier, sloshing-ion A-cell and use the analytic distribution function suggested by $R$. H. Cohen ${ }^{I-1}$ for the thermal jons. This distribution, being continuous across the separatrix, is less likely to lead to potential sheaths. For the thermal electrons, we use an analytic distribution that is again continuous across the separatrix. The general configuration and notation is depicted in Fig. I-1. In what follows, we only consider the region between $B_{M}$ and $\Phi_{C}$.

We first consider the thermal electrons in the well region (between $B_{0}$ and $B_{c}$ ) whose phase space is carved up as shown in fig. I-2 (note $\phi=\mid e(\phi)$.

To model the hot trapped electrons and the warm passing e?ectrons, we approximate the electron distribution as follows:

$$
f=\frac{1}{\sqrt{\pi}} \exp \left(-\varepsilon-\phi_{0}\right), \varepsilon>-\phi_{0} ;
$$

and

$$
f=\frac{1}{\sqrt{\pi}} \exp \left(-\varepsilon \tau-\phi_{0} \tau\right), \varepsilon<-\phi_{0} ;
$$

where

$$
i=\mathrm{T}_{\text {warm }} / \mathrm{T}_{\text {hot }} \text {. }
$$

Thus, for ease of analysis, we assume that the temperatures of passing and weakly trapped electrons are the same. Also, for the same reason, we assume the more deeply trapped electrons and the more deeply trapped Yushmanor electrons have the same temperature. 


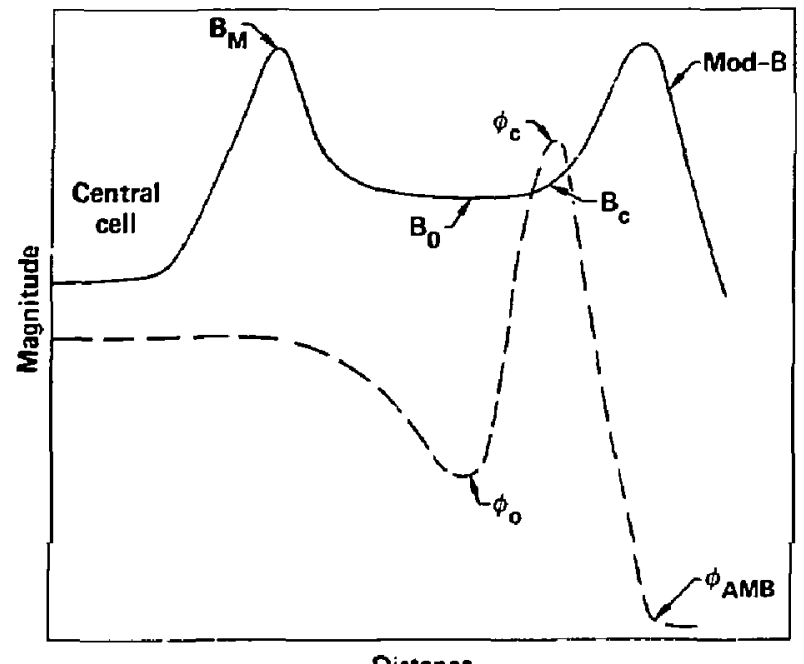

Fig. 1-1. Magnetic-field strength (solid line) =r.d ambipolar potential (dashed line) for a typical thermal-barrier cell, i a tandem mirror.

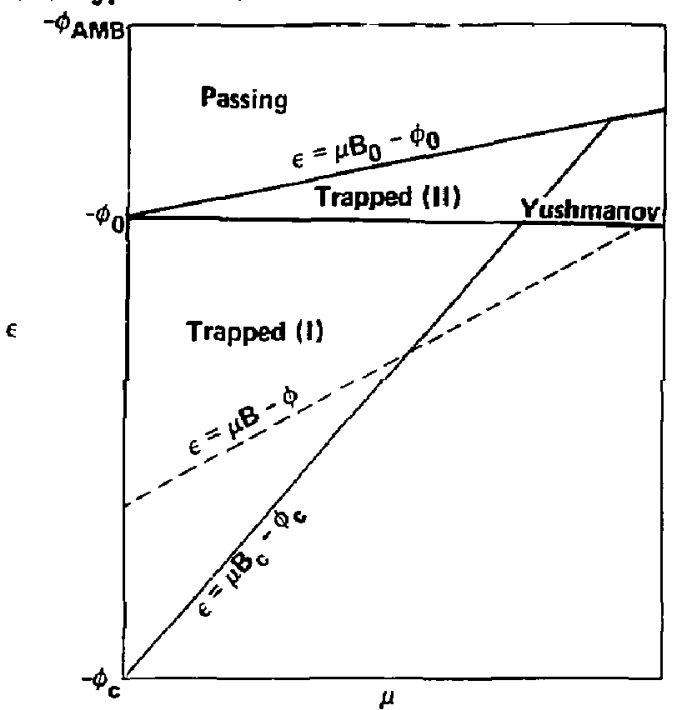

Fig. I-2. Thermal-electron phase space. Electrons in the regions labeled Trapped (I) and Trapped (II) are confined by the potential peak about $\phi_{C}$. Electrons in the shaded region (Yushmanov electrons) are confined by a combination of magnetic forces and the ambipolar electric field. 
The thermal electron density is then

$$
\begin{aligned}
n_{p}= & \frac{1}{\sqrt{\pi}} \int_{-\phi_{0}}^{-\phi_{A M B}} d \varepsilon \exp \left(-\varepsilon-\phi_{0}\right) \int_{0}^{\varepsilon+\phi} \frac{d \mu B}{\sqrt{\varepsilon-\mu B+\phi}} \\
= & \frac{2}{\sqrt{\pi}}\left[\left(\phi-\phi_{0}\right)^{1 / 2}-\exp \left(\phi_{A M B}-\phi_{0}\right)\left(\phi-\phi_{A M B}\right)^{1 / 2}\right] \\
& +\overline{\operatorname{erfc}}\left(\phi-\phi_{0}\right)=\exp \left(\phi_{A M B}-\phi_{0}\right) \overline{\operatorname{erfC}}\left(\phi-\phi_{A M B}\right) .
\end{aligned}
$$

and

$$
\begin{aligned}
n_{t} & =\frac{1}{\sqrt{\pi}} \int_{\omega \phi}^{-\phi_{0}} d \varepsilon \exp \left[-\tau\left(\varepsilon+\phi_{0}\right)\right] \int_{0}^{E+\phi} \frac{d \mu B}{\sqrt{\varepsilon-\mu B+\phi}} \\
& =-\frac{2}{\sqrt{\pi}} \frac{\left(\phi-\phi_{0}\right)^{1 / 2}}{\tau}+\frac{1}{\tau / 2} \overline{\operatorname{erf}}\left[\left(\phi-\phi_{0}\right) \tau\right]
\end{aligned}
$$

where we have defined

$$
\begin{aligned}
& \overline{\operatorname{erf}}\left(u^{2}\right)=\frac{2}{\sqrt{\pi}} \exp \left(u^{2}\right) \int_{0}^{u} d t e^{-t^{2}} \\
& \overline{\operatorname{erfc}}\left(u^{2}\right)=\frac{2}{\sqrt{\pi}} \exp \left(u^{2}\right) \int_{u}^{\infty} d t e^{2 t}
\end{aligned}
$$

Next consider the phase space for the thermal ions (see Fig. I-3).

The dashed line is the $v_{11}=0$ line for positive potentials and the wavy line is the same for negative potentials. Following $\mathrm{B}$. H. Cohen ${ }^{I-1}$ we write the distribution for the passing ions as

$$
f_{p}=e^{-\varepsilon}
$$

and for the trapped ions as

$$
f_{T}=\exp \left(\frac{\varepsilon-\alpha \mu B_{M}}{\alpha-T}\right)
$$




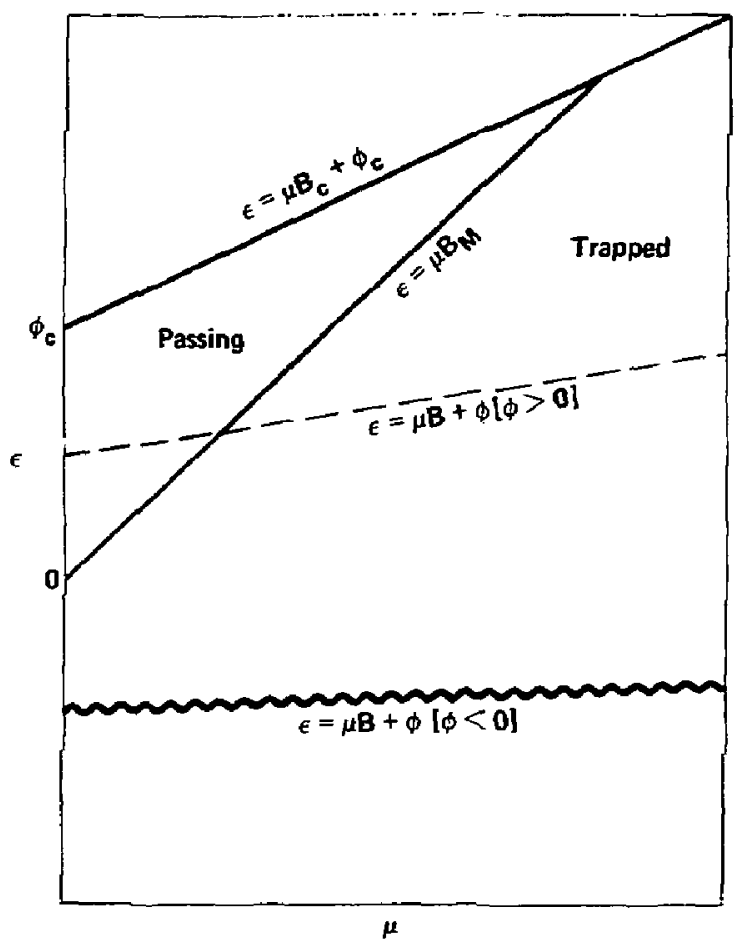

Fig. 1-3. Thermal-ion pinase space. 
which is continuous on the separatrix $\left(\varepsilon=1 \mathbb{B}_{M}\right)$. The density of trapped particles increases continuously as a goes from 1 to too and then from $-\infty$ to 0 . The limit $\alpha=\gamma$ corresponds to zero trapped ions, while $\alpha=0$ corresponds to a completely filled barrier.

We now compute the ion densities for the passing ions. First, for negative potentials we have

$$
n_{\mathrm{p}}^{*}=\frac{1}{\sqrt{\pi}} \int_{0}^{\phi_{\mathrm{p}} / R} d y \int_{\mathrm{yR}}^{\phi_{\mathrm{C}}+\mathrm{yR} / \mathrm{R}_{\mathrm{c}}} d \varepsilon \exp (-\varepsilon)(\varepsilon-y-\phi)^{1 / 2},
$$

where here, and in what follows, we have the following definition::

$$
\begin{aligned}
& y=\omega B, \\
& R=B_{M} / B, \\
& \Psi=\phi\left(\frac{R}{R-1}\right), \\
& \Phi_{p}=\phi_{C} \frac{R_{C}-1}{R_{C}}, \\
& x=\frac{\phi}{a-T} .
\end{aligned}
$$

Next writing $\varepsilon-y-\phi=x^{2}$, we have

$$
n_{p}^{-}=\frac{2}{\sqrt{\pi}} \int_{0}^{\phi_{p} / R} d y \exp (-y) \int_{[y(R-1)-\phi]^{1 / 2}}^{\left[y\left(R / R_{c}-1\right)+\phi_{c}-\phi\right]^{1 / 2}} d x \exp \left(-x^{2}-\phi\right) .
$$

and integrating by parts on $y$, we obtain

$$
\begin{aligned}
n_{p}^{-}= & \overline{\operatorname{erfc}}(-\phi)-\overline{\operatorname{erfc}}\left(\phi_{c}-\phi\right) \mathrm{e}^{-\phi c} \\
& -\left(\frac{R-1}{R}\right)^{1 / 2}\left[\overline{\operatorname{erfc}}(-\psi)-e^{-\phi_{p}} \overline{\left.\operatorname{erfc}\left(\phi_{\mathrm{p}}-\psi\right)\right]}\right. \\
& +\frac{1}{\sqrt{\pi}}\left(1-\frac{R_{c}}{R}\right)^{1 / 2} \int_{0}^{\phi_{p} / R_{c}} d t e^{-t} \frac{1}{\left.\sqrt{t+\frac{R}{R-R_{C}}\left(\phi_{c}-\phi\right)}\right]^{D / 2}} .
\end{aligned}
$$


which can be written

$$
\begin{aligned}
& n_{p}^{-}=\overline{\operatorname{erfc}}(-\phi)-e^{-\phi_{c}} \overline{\operatorname{erfc}}\left(\phi_{c}-\phi\right) \\
& -\left(\frac{R-1}{R}\right)^{1 / 2}\left[\overline{\operatorname{erfc}}(-\psi)-e^{-\phi_{p}} \overline{\operatorname{erfc}}\left(\phi_{p}-\psi\right]\right] \\
& +\left(1-\frac{R_{c}}{R}\right)^{1 / 2}\left\{e^{-\phi_{c}} \overline{\operatorname{erfc}}\left[\frac{R}{R-R_{c}}\left(\phi_{c}-\phi\right)\right]-e^{-\phi_{p}} \overline{\operatorname{erfc}}\left(\frac{R-1}{R-R_{c}}\right)\left(\phi_{p}-\psi\right)\right\} .
\end{aligned}
$$

Next for positive potentials, we have

$$
\begin{aligned}
n_{p}^{+}= & \frac{1}{\sqrt{\pi}}\left[\int_{0}^{\phi /(R-1)} d y \int_{y+\phi}^{y R / R_{C}+\phi_{c}} d \varepsilon e^{-\varepsilon}(\varepsilon-\phi-y)^{-1 / 2}\right. \\
& \left.+\int_{\phi / R-T}^{\phi_{p} / R} d y \int_{y R}^{\phi^{+y R / R} c} d \varepsilon e^{-\varepsilon}(\varepsilon-\phi-y)^{-1 / 2}\right] .
\end{aligned}
$$

Consequently, for the passing ions we have

$$
\begin{aligned}
& n_{p}=\left(\left(1-\frac{R_{C}}{R}\right)^{1 / 2}\left\{e^{-\phi_{C}} \overline{\operatorname{erfc}}\left[\frac{R}{R-R_{C}}\left(\phi_{C}-\phi\right)\right]-e^{-\phi_{p}} \overline{\operatorname{erfc}}\left[\frac{R-1}{R-R_{C}}\left(\phi_{D}-\psi\right)\right]\right\}\right. \\
& \overline{\operatorname{erf}}\left(\phi_{c}-\phi\right)-\left(\frac{R}{R-T}\right)^{1 / 2} e^{-\phi_{p}} \overline{\operatorname{erf}}\left(\phi_{p}-\psi\right) \quad \phi>0
\end{aligned}
$$

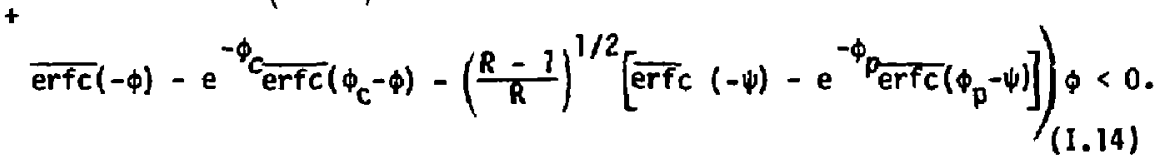


Next turn to the trapped plasma. Consider $\phi>0$, then we have

$$
\begin{aligned}
n_{t r}^{+}= & \frac{1}{\sqrt{\pi}} \int_{\phi / R-1}^{\phi_{p} / R} d y \exp (-\alpha y R / \alpha-1) \int_{y+\phi}^{y R} d \varepsilon \exp (\varepsilon / \alpha-1)(\varepsilon-\phi-y)^{-1 / 2} \\
& +\frac{1}{\sqrt{\pi}} \int_{\phi_{p} / R}^{\infty} d y \exp (-\alpha y R / \alpha-1) \int_{y+\phi}^{y R / R_{c}+\phi_{c}} d \varepsilon \exp (\varepsilon / \alpha-1)(\varepsilon-\phi-y)^{1 / 2}
\end{aligned}
$$

Again transforming the square root away, we obtain

$$
\begin{aligned}
& n_{t r}^{+}=\frac{2}{v \pi}\left[\int_{\phi / R-1}^{\phi p / R} d y \exp \left(-y \frac{\alpha R-1}{\alpha-1}+\frac{\phi}{\alpha-T}\right) \int_{0}^{[y(R-1)-\phi]^{1 / 2}} d x \exp \left(\frac{x^{2}}{\alpha-T}\right)\right. \\
& \left.+\int_{\dot{\varphi}_{p} / R}^{\infty} d y \exp \left(-y \frac{\alpha-1}{\alpha-1}+\frac{\phi}{\alpha-1}\right) \int_{0}^{\left[y\left(R / R_{c}-1\right)+\phi_{c}-\phi\right]^{1 / 2}} d x \exp \left(\frac{x^{2}}{\alpha-1}\right)\right] .
\end{aligned}
$$

Next, integrating by parts, we find that the integrated parts cancel, leaving

$$
\begin{aligned}
n_{t r}^{+}= & \frac{\alpha-1}{\alpha R-1} e^{-\phi_{p}}\left(\frac{R-I}{R}\right)^{1 / 2}\left(\phi_{p}-\psi\right) \\
& +\frac{1}{\sqrt{\pi}} \int_{\phi_{p} / R}^{\infty} d y \exp \left(-y \frac{\alpha R-1}{\alpha-1}+\frac{\phi}{\alpha-1}\right)\left(1-\frac{R_{c}}{R}\right)^{1 / 2} \\
& \left.\frac{R}{\Omega_{c}}\left(\frac{\alpha-1}{\alpha R_{c}-T}\right)^{1 / 2} \frac{1}{\left(y+\frac{\phi_{C}-\phi}{R / R_{c}-T}\right)^{1 / 2}} \exp \left[\frac{y\left(R / R_{c}-1\right)+\phi_{c}-\phi}{\alpha-1}\right]\right\} .
\end{aligned}
$$


Finally, combining terms and going through the same algebra for $\phi<0$, we obtain

$$
\begin{aligned}
& n_{t r}=\left(\frac{\alpha-1}{\alpha R-1}\right) \int^{-\phi_{p}}\left(1-R_{c} / R\right)^{1 / 2}\left(\frac{\alpha-1}{\alpha R_{c}-1}\right)^{1 / 2} \overline{\operatorname{erfc}}\left[\frac{\alpha R_{c}-1}{\alpha-1} \frac{R-1}{R-R_{c}}\left(\phi_{p}-\psi\right)\right] \\
& \left(\frac{R-1}{R}\right)^{1 / 2} \mathrm{e}^{-\phi_{p}} \overline{\mathrm{erf}}\left(\phi_{p}-\psi\right) \\
& \left.\left(\frac{R-1}{R}\right)^{l / 2}\left[\overline{\operatorname{erfc}}(-\psi)-e^{\phi_{p}} \overline{\operatorname{erfc}}\left(\phi_{p}-\psi\right)\right]+2\left(\frac{\alpha-1}{\pi}\right)^{1 / 2} D\left(x^{1 / 2}\right)\right\}, \phi<0
\end{aligned}
$$

where the Dawson integral is defined by

$$
D(x)=e^{-x^{2}} \int_{0}^{x} d t e^{t^{2}}
$$

In terms of $Z$ functions, we have

$$
\begin{aligned}
& \frac{2}{\sqrt{\pi}} D(x)=i e^{-x^{2}}-\frac{1}{\sqrt{\pi}} Z(x), \\
& \operatorname{erfc}\left(x^{2}\right)=-\frac{i}{\sqrt{\pi}} Z(i x),
\end{aligned}
$$

which defines the analytic continuation $\left[\right.$ e.g., consider $2(\alpha-1 / \pi)^{1 / 2} 0\left(x^{1 / 2}\right)$ for $a<0$, note $0<a<1$ is forbidden],

$$
2\left(\frac{\alpha-1}{\pi}\right)^{1 / 2} v\left(x^{1 / 2}\right) \rightarrow(1-\alpha)^{1 / 2} \overline{\operatorname{erf}}(x) \text {. }
$$

In the remainder of this paper we show several representative solutions for the barrier potential and thermal-ion density profile. The sloshing-ion distribution was chosen to satisfy

$$
n_{S L O S H}=\left(\frac{1-1 / R}{1-1 / R^{\star}}\right)^{3 / 2} \exp \left[-\left(\frac{R^{*}}{R}-1\right)^{2} / \Delta^{2}\right] \exp \left[+\left(\frac{R^{*}}{R_{c}}-1\right)^{2} / \Delta^{2}\right](1
$$

where $R^{\star}$ is chosen to force " $S L O S H$ to peak at $R_{:}$and $\Delta$ is fixed by the relative sloshing-ion density at the midplane $\left(R_{m}\right)$. For the magnetically trapped ECRH electrons, we choose 


$$
n_{E C R H}=n_{E C R H}(0)\left(\frac{1-1 / R}{1-1 / R_{M}}\right)^{3 / 2} \text {, }
$$

where $n_{E C R H}(0)$ is determined by forcing charge neutrality together with one further condition needed for closure. A convenient choice is the density ratio of thermal electrons to ECRH electrons at the midplane.

A comparison of the two figures shows the following parameters in common:

$$
\begin{aligned}
& \frac{n_{S L O S H}(0)}{n_{S L O S H}\left(R^{*}\right)}=0.3 \text {. } \\
& \frac{n_{t h . e^{(0)}}}{n_{E C R H}(0)}=0.1 \\
& \frac{T_{e, w a r m}}{T_{i}}=1 \begin{array}{l}
\text { (temperature ratio of thermal } \\
\text { electrons to thermal ions) }
\end{array}, \\
& \frac{T_{e, \text { warm }}}{T_{\text {e, hot }}}=\tau=0.2 \text {. } \\
& \frac{{ }_{C C}}{n_{S L O S H}\left(R^{*}\right)}=1 \text {. } \\
& n_{t h . j}(0) / n_{c c}=0.085 \text {, } \\
& R_{M}=5 \text {, } \\
& \phi_{A M B}=5 \text {. }
\end{aligned}
$$

Note that since the separatrix for the thermal electrons is energy dependent $v=3 / 2$ (R. H. Cohen's notation, Ref. I-l). To mock up a more realistic $v=1 / 2$, we raise the $T_{e, \text { hot }}$ to get a higher plug potential $\left(\phi_{C}\right)$. In Fig. $I-4$, we have various profiles for $\alpha=1.5$, which results in a $g\left(R_{M}\right)=1.8$ and $n_{\text {th.j }}(0) / n_{c c}=0.085$. In Fig. I-5, we have the same profiles for $\alpha=3$, which results in a $g\left(R_{W}\right)=6.5$ and $n_{t h . j}(0) / n_{c c}=2.4$. We use Logan's def inition $I-2$ of $g$, namely $g(R)=n_{t h . j}(R) / n_{p i}(R)$. 


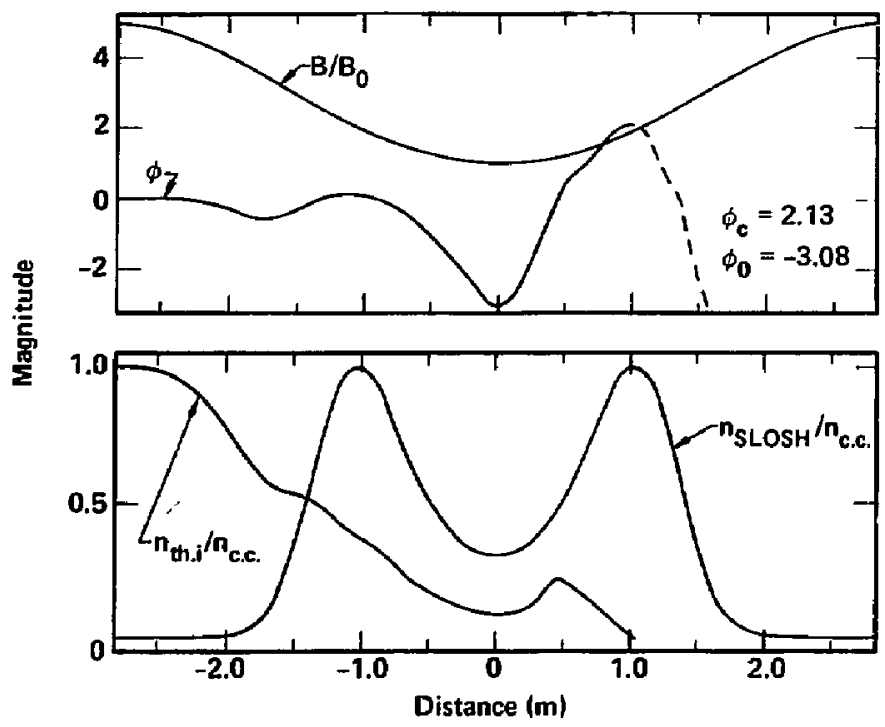

Fig. 1-4. Profiles of Mod-B, ambipolar potential $\phi$; thermal-ion density $n_{\text {th.i }}$; and sloshing-ion density $n_{\text {SLOSH }}$ for $\alpha=1.5$.

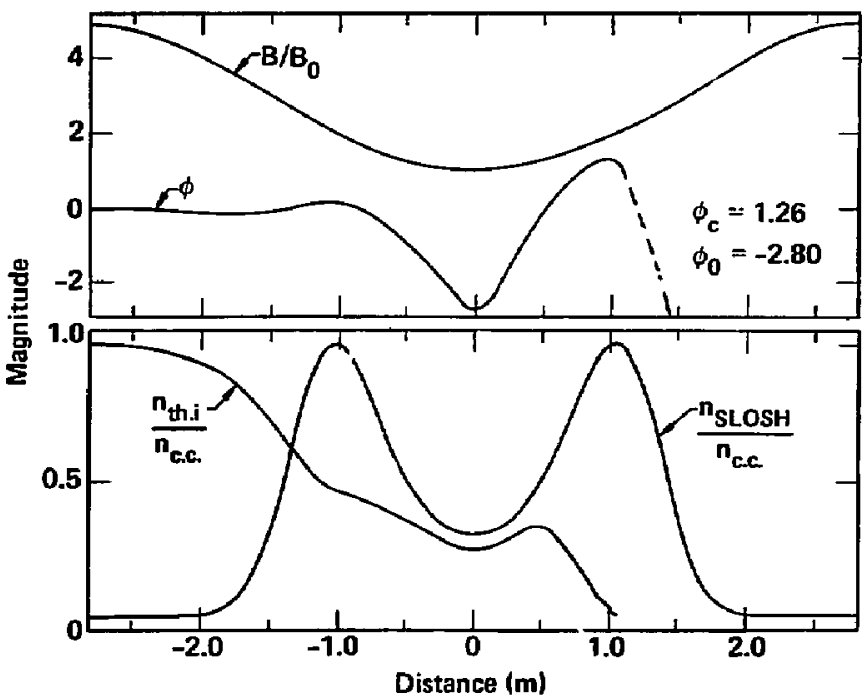

Fig. 1-5. The same profiles as in Fig. I-4 but with $\alpha=3$. 
Note that in this case the barrier potential is roughly symetric about its minimum. That is, there is no resemblance of a sheath. Using the analytic models for continuous distrbution across separatrices has removed the sheath. It should be emphasized that these are approximate analytic models for equilibrium distribution. Except for extreme cases [too few trapped thermal ions $(\alpha<1.1)$ or too much necking off $\left(n_{\text {th. }} e^{\left.(0) / n_{E C R H}<0.025\right)}\right)$, they give rise to continuous potentials and densities and are thus quite useful for obtaining as profiles of $n$ and $\phi$ for use in composite calculations. Ion microstability is one such case. Using numerical profiles from Fokker-Planck codes would increase computer times by at least an order of magnitude (probably two orders). This is of course the trade off, the potentially inaccurate profiles for computer speed and reliability. In Fig. I-6 we plot various equifibrium quantities as a function of $g\left(R_{M}\right)$.

\section{REFERENLES}

I-1. R. H. Cohen, Nuclear Fusion 21, 209 (1981).

I-2. D. E. Baldwin and B. G. Logan, Phys. Rev. Lett. 43, 1318 (1979). 


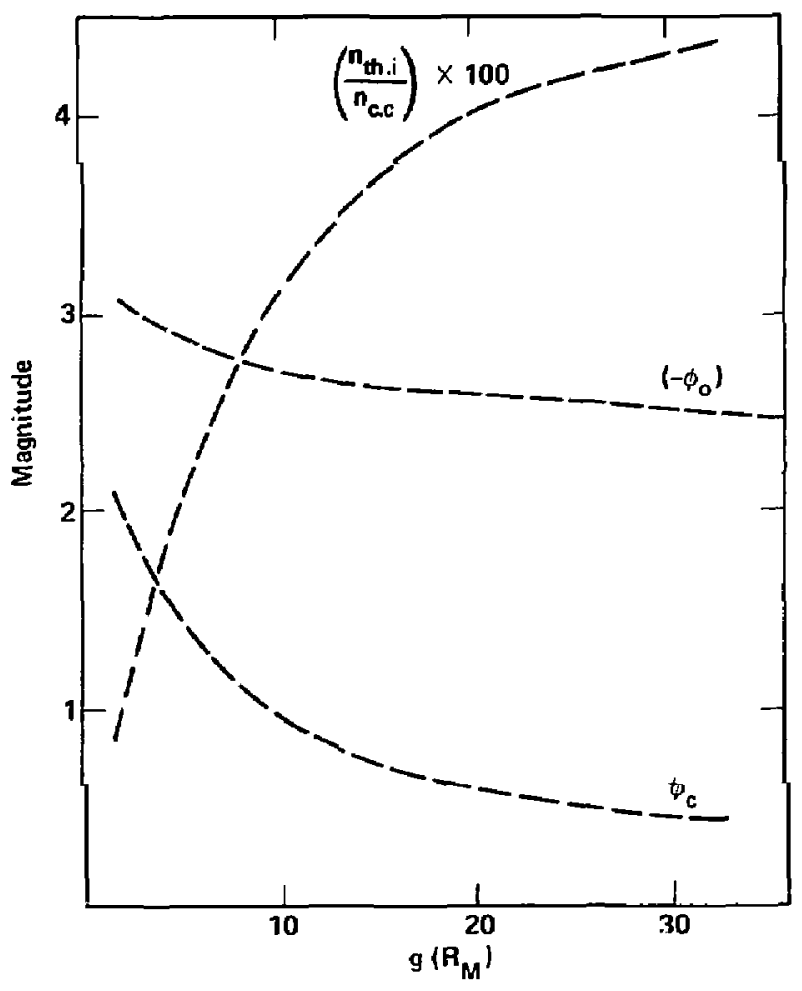

Fig. I-6. Various equilibrium quantities as a function of $g\left(R_{M}\right)$. 


\section{DISCLAIMER}

This dacumeat was prepared 25 an account of work sponsored by an agency of the Uniled States Goremmeat. Neither the United States Government nor the University of Calitomia nor any of their employees, makes any warrany, express or implied, or ascumes any lezal liability of responsibility for the aceurrey, eompleteness, or usefulecs of any iuformation, apanatus, proluct, or process disclosed, of represents that its use would not infringe privately on ned rights, Reference herein to any spteific commercinl products, process,

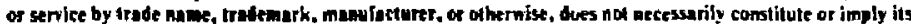
eadorsemeat, recommendation, or favoriog by the Linited Stutes Govemment or the Linivetsity of Californin. The vlews and opialons of anthors expresurd berein do wot necessarily sate of reflect those of the lnifed Siates Covernment thereol. and shall not be uxed for advertislac of product cndorsement purposes.

$$
\begin{aligned}
& \text { Printed un the United Siater of Amesucis } \\
& \text { Nattennal Tachnsal Infurmulisun Service } \\
& \text { U.S. Deparmenti ar Cummeror }
\end{aligned}
$$

\begin{tabular}{|c|c|c|c|}
\hline Puge Range & $\begin{array}{l}\text { Domestic } \\
\text { Price }\end{array}$ & Page Range & $\begin{array}{l}\text { Domestic } \\
\text { Price }\end{array}$ \\
\hline $001-025$ & 55.00 & $326 \cdot 350$ & 518.00 \\
\hline $026-050$ & 6.00 & $351-375$ & 19.00 \\
\hline $051-075$ & 7.00 & $376-400$ & 20.00 \\
\hline $076-100$ & 8.00 & $401-425$ & 21.00 \\
\hline $101-125$ & 9.00 & $426-450$ & 22.00 \\
\hline $126-150$ & 10.00 & $451-475$ & 23.00 \\
\hline 151-175 & 11.00 & $476-500$ & 24.00 \\
\hline $176 \cdot 200$ & 12.00 & $501-525$ & 25.00 \\
\hline $201-225$ & 13.00 & $526-550$ & 26.00 \\
\hline $226-250$ & 14.00 & $551-525$ & 27.00 \\
\hline $251-275$ & 15.00 & $\$ 26-550$ & 28.00 \\
\hline $276 \cdot 300$ & 16.00 & 601-up 1 & \\
\hline $301-325$ & 17.00 & & \\
\hline
\end{tabular}

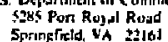

$$
\begin{aligned}
& \text { Prove Pronted Corp? } \$
\end{aligned}
$$

${ }^{1}$ Add 2.00 tor each additional 25 page increment from 601 pages up. 
Technical Information Deparment - Lawrence Livermore Laboratory University of California - Livermort. California 94550 